\title{
Chemical Analysis of Firearm Discharge Residues Using Laser Induced Breakdown Spectroscopy
}

\author{
Courtney Helen Vander Pyl \\ West Virginia University, chv0002@mix.wvu.edu
}

Follow this and additional works at: https://researchrepository.wvu.edu/etd

\section{Recommended Citation}

Vander Pyl, Courtney Helen, "Chemical Analysis of Firearm Discharge Residues Using Laser Induced Breakdown Spectroscopy" (2019). Graduate Theses, Dissertations, and Problem Reports. 4058. https://researchrepository.wvu.edu/etd/4058

This Thesis is protected by copyright and/or related rights. It has been brought to you by the The Research Repository @ WVU with permission from the rights-holder(s). You are free to use this Thesis in any way that is permitted by the copyright and related rights legislation that applies to your use. For other uses you must obtain permission from the rights-holder(s) directly, unless additional rights are indicated by a Creative Commons license in the record and/ or on the work itself. This Thesis has been accepted for inclusion in WVU Graduate Theses, Dissertations, and Problem Reports collection by an authorized administrator of The Research Repository @ WVU. For more information, please contact researchrepository@mail.wvu.edu. 
Chemical Analysis of Firearm Discharge Residues Using Laser Induced Breakdown Spectroscopy

Courtney H. Vander Pyl

Thesis submitted

to the Eberly College of Arts and Sciences at West Virginia University

in partial fulfillment of the requirements for the degree of

Master of Science in

Forensic and Investigative Sciences

\section{Tatiana Trejos, Ph.D., Chair}

Keith Morris, Ph.D.

Luis Arroyo, Ph.D.

Casey Jelsema, Ph.D.

\section{Department of Forensic and Investigative Sciences}

Morgantown, West Virginia

2019

Keywords: Firearm discharge residues, Gunshot residue, Laser Induced Breakdown Spectroscopy, Distance determination, Forensics

Copyright 2019 Courtney Vander Pyl 


\begin{abstract}
Chemical Analysis of Firearm Discharge Residues Using Laser Induced Breakdown Spectroscopy

Courtney Vander Pyl
\end{abstract}

According to the Gun Violence Archive, in 2017, firearms were involved in 57,134 criminal incidents in the United States. The detection of firearm discharge residues (FDR), including inorganic and organic gunshot residues, can provide essential information in such investigations. For instance, when the question of suicide or murder arises, estimating the barrel to victim distance plays a critical role in the outcome of the case. In addition, clothing, wounds and other target materials are often inspected to determine if a bullet has produced an entry or exit orifice. Currently, the most common method for distance determination and identification of bullet entrance holes is by chemical colorimetric tests that react in the presence of nitrites or lead. Although these color tests are widely used in forensic laboratories, the major pitfall is their variability and poor selectivity for gunshot residues. Dark or bloody items significantly diminish the efficacy of these assays, they are difficult to perform on non-movable or large objects, and false positives can be derived from oil, dirt, and other common contaminants.

The chance of outside sources affecting the color distribution pattern has led to the exploration of alternative instrumental methods for estimating a shooting distance, including Atomic Absorption Spectroscopy (AAS), Fourier Transform Infrared Spectroscopy (FTIR), X-ray fluorescence (XRF), and Atomic Force Microscopy (AFM). Nevertheless, these instrumental methods have limitations for GSR analysis, as well, such as destruction of the sample, long time of analysis, or complex sample preparation. Therefore, there is a critical need for the development of an analytical method that is sensitive to GSR, fast, practical, non-invasive, objective, and accurate.

The primary goal of this study was to investigate the capabilities of LIBS for shooting distance determination and identification of FDR on substrates of interest. The central hypothesis of this research was that Laser-Induced Breakdown Spectroscopy would improve the scientific reliability of the detection and observation of gunshot residues. This assumption was based on the ability of LIBS to perform simultaneous multi-elemental detection at low ppm levels, LIBS' superior selectivity, and the potential for confirmation of numerous emission species per analyte. 
To assess the forensic utility of LIBS, the method was developed and validated for the analysis of substrates commonly found during firearm-related crimes. Residues from different ammunitions and firearms were analyzed off 133 fabrics, glass, drywall, and wooden samples. Statistical methods, like principal component analysis and multivariate discriminant analysis were performed to estimate shooting distances and identify the presence of GSR residues. Color tests lead to misclassification of 9 out of 35 unknown shooting distances (26\%), while the LIBS method correctly classified $100 \%$ of the unknown distance testing samples by Discriminant Analysis. Additionally, LIBS was able to correctly identify elemental profiles of gunshot residues from all standard ammunitions deposited on clothing, drywall, glass, and wood.

LIBS allowed for rapid and accurate chemical mapping of GSR patterns on pieces of evidence typically found at a crime scene. Chemical imaging of lead, barium, and antimony provided more objective approaches to estimation of shooting distance and bullet hole identification, compared to color tests. Moreover, LIBS provided enhanced detection of standard ammunitions and partial detection of lead-free ammunitions. However, some challenges still exist with accurate detection and identification of non-toxic ammunitions.

The proposed method is anticipated to aid in crime scene reconstruction of criminal events involving firearms. The superior capabilities of LIBS analysis, compared to current practice, increases the certainty on these examinations and enhances the reliability of the information used during the investigative stages and when the evidence is presented in a court of law. 


\section{ACKNOWLEDGEMENTS}

First, I would like to express my overwhelming gratitude to my research advisor and committee chair, Dr. Tatiana Trejos, for her mentorship and guidance over the last two years. None of this would have been possible without her support, reassurance, and encouragement.

I would like to thank my committee members Dr. Keith Morris, Dr. Luis Arroyo, and Dr. Casey Jelsema for providing thoughtful insight and advice throughout the process.

I would also like to thank my fellow graduate and undergraduate research group members who have greatly helped me. Especially Korina Menking-Hoggatt who always listened to my struggles, shared in my frustration of uncooperative instruments, and celebrated all the little achievements with me.

Finally, I would like to thank my parents, my sister Chelsea, my boyfriend Cameron, and all of my friends for their endless encouragement and love throughout this whole experience. Thank you for always believing in me. 


\section{TABLE OF CONTENTS}

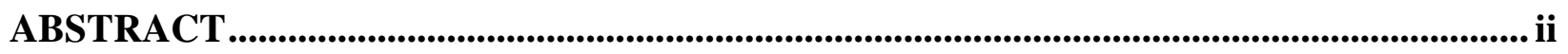

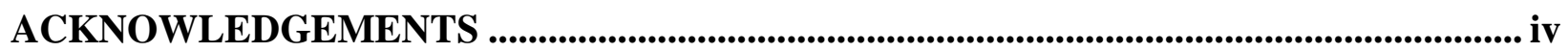

LIST OF FIGURES ........................................................................................................... viii

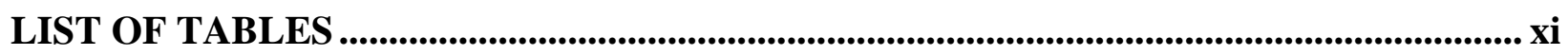

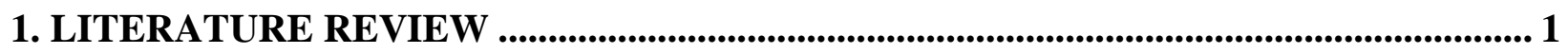

1.1. Firearm Discharge Residues and Gunshot Residues ....................................................... 1

1.2. Scanning Electron Microscopy-Energy Dispersive Spectroscopy Analysis ............................ 3

1.3. Physical Examination and Colorimetric Tests for FDR Detection ............................................ 6

1.4. Modified Griess Test........................................................................................................ 7

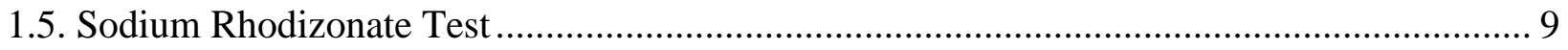

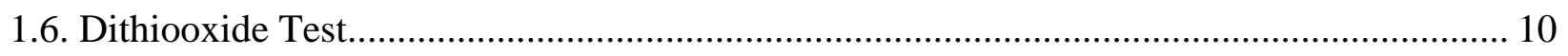

1.7. Current Challenges and Advances on GSR Detection....................................................... 11

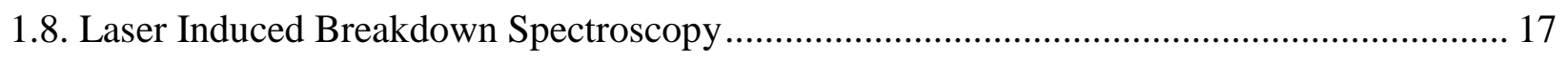

2. OBJECTIVES OF OVERALL PROJECT ............................................................................... 22

3. CHAPTER 1: THE DEVELOPMENT, OPTIMIZATION, AND VALIDATION OF LIBS FOR SHOOTING DISTANCE DETERMINATION ...................................................... 24

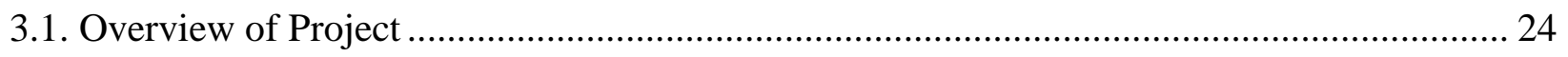

3.2. Spectrochemical Mapping Using Laser Induced Breakdown Spectroscopy as a More Objective Approach to Shooting Distance Determination.......................................................... 25

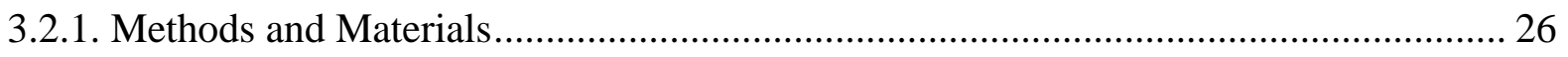

3.2.1.1. Firearms and Ammunitions............................................................................. 26

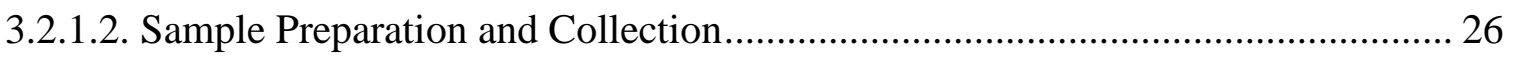

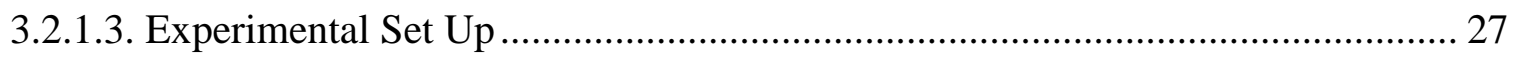

3.2.1.3.1. LIBS Instrumentation, Parameters, and Method......................................... 27

3.2.1.3.2. LIBS Data Analysis ........................................................................... 29

3.2.1.3.3. Color Tests and Physical Examinations.................................................. 29

3.2.1.3.3.1. Color Test Reagents .................................................................... 29

3.2.1.3.3.2. Physical Measurements................................................................... 30

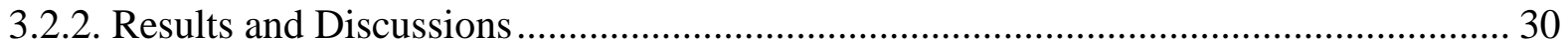

3.2.2.1.Firing Distance Prediction Using LIBS Analysis and Multivariate Methods....... 30 
3.2.2.1.1. LIBS Method Optimization and Assessment of Selectivity and Limits of

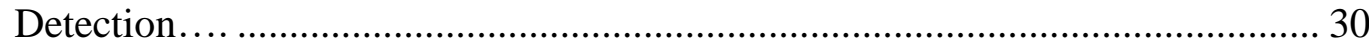

3.2.2.1.2. Chemical Maps for Shooting Distance Determinations .............................. 33

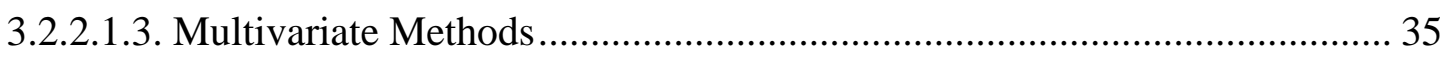

3.2.2.2. Firing Distance Predictions using Color Tests..................................................... 38

3.2.2.2.1. Interpretation of Physical and Visual Examination and Chemical Color

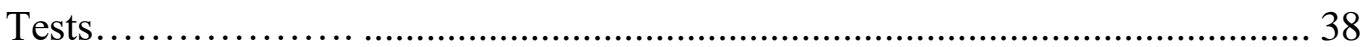

3.2.2.3.True Shooting Distance: Assessment of Accuracy of the Methods...................... 39 3.3. Spectrochemical Mapping Using LIBS for Shooting Distance Determination on Bloody

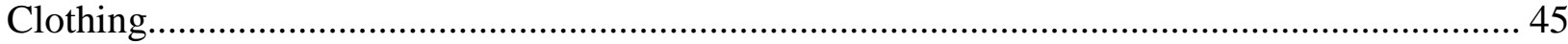

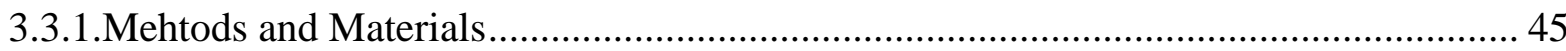

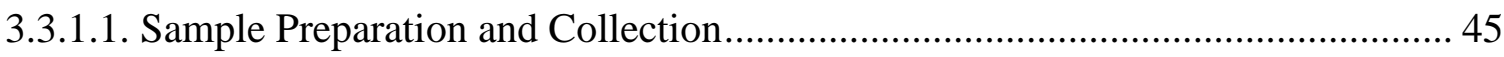

3.3.1.2. Application of Blood to White Clothing Samples ............................................... 45

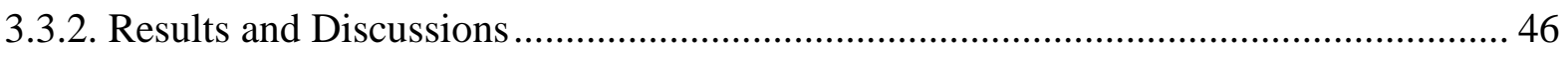

3.3.2.1. Firing Distance Prediction Using LIBS Analysis and Multivariate Methods...... 46

3.3.2.1.1. Chemical Maps for Shooting Distance Determinations ............................. 46

3.3.2.1.2. Multivariate Methods................................................................................. 48

3.3.2.2. Firing Distance Predictions using Color Tests................................................. 50

3.3.2.2.1. Interpretation of Physical and Visual Examination and Chemical Color

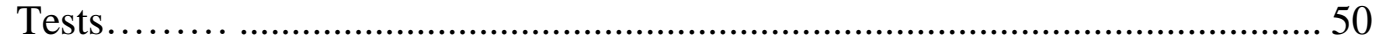

3.3.2.3. True Shooting Distance: Assessment of Accuracy of the Methods...................... 54

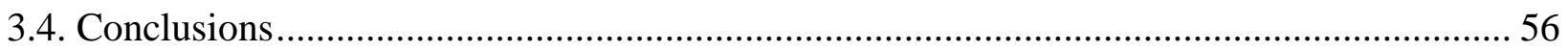

\section{CHAPTER 2: THE DEVELOPMENT OF A FAST CHEMICAL MAPPING METHOD} FOR THE IDENTIFICATION OF ENTRANCE HOLES................................................. 58

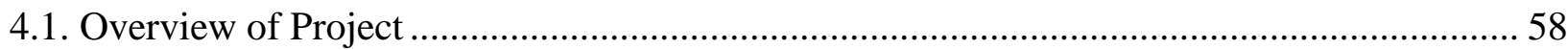

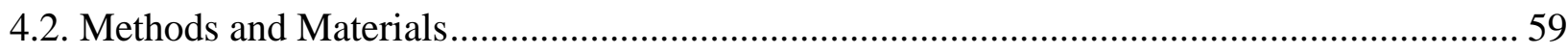

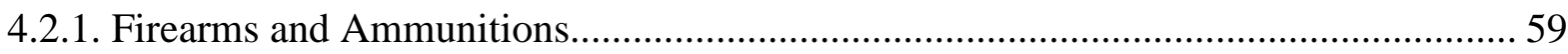

4.2.2. Substrate Preparation and Collection ........................................................................... 59

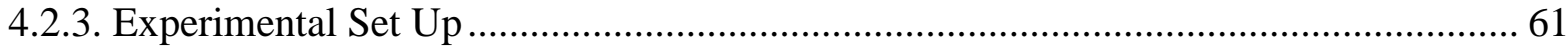

4.2.4. LIBS Instrumentation, Parameters, and Method.......................................................... 62

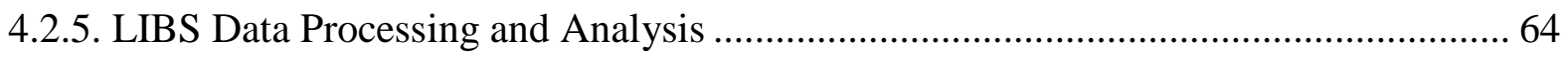

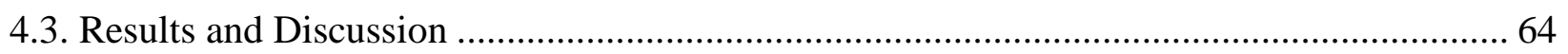

4.3.1.Assessment of Adhesive Selection for Transfer of Gunshot Residues.......................... 64

4.3.2. Assessment of Shooting Substrate Selection .................................................................. 67 
4.3.3. Collection of Gunshot Residues from Wood, Drywall, and Glass Samples............... 69

4.3.4. Assessment of LIBS Method and Chemical Maps for Bullet Hole Identification ....... 72

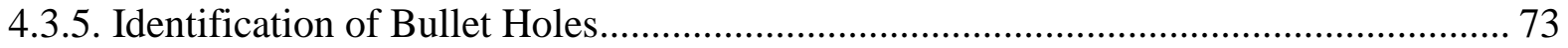

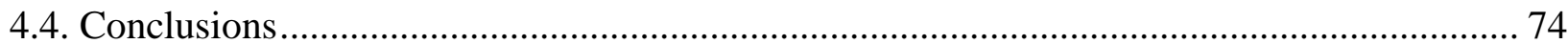

5. CHAPTER 3: THE EVALUATION OF THE USE OF LIBS FOR GSR DETECTION OF MULTIPLE PRIMERS AND BULLET TYPES .................................................. 76

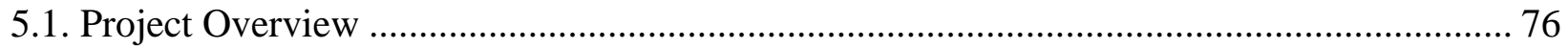

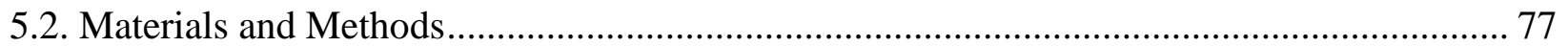

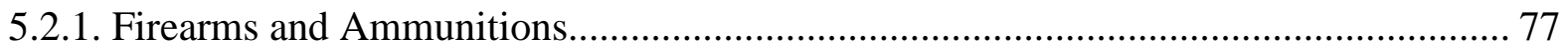

5.2.2. Sample Preparation and Collection...................................................................... 78

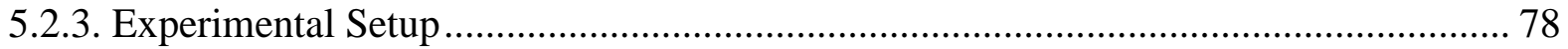

5.2.4. LIBS Instrumentation, Parameters, and Methods ............................................. 78

5.2.5. LIBS Data Processing and Analysis ........................................................................ 78

5.2.6. Physical Examinations and Color Tests............................................................ 80

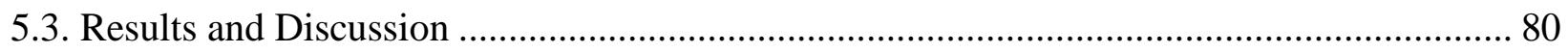

5.3.1. Visual Characteristics of Firearm Discharge Residues............................................. 80

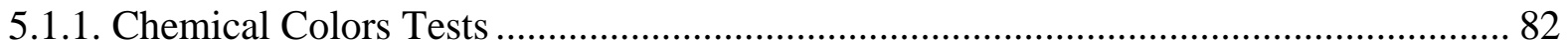

5.1.2. Identification of Elements Using Laser Induced Breakdown Spectroscopy .............. 84

5.1.3. Additional Testing Methods for Confirmation of Chemical Profiles ......................... 85

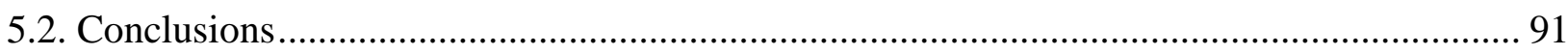

6. OVERALL CONCLUSIONS AND RECOMMENDATIONS .................................... 93

6.1. Conclusions of Proposed Methodologies.................................................................... 93

6.2. Recommendations and Future Expansion of Work ..................................................... 94

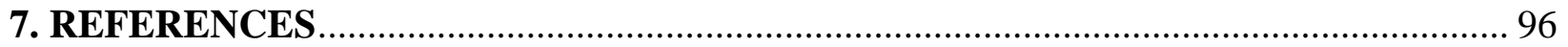

APPENDIX I. ADDITIONAL PLOTS AND HEAT MAPS ........................................... 102

APPENDIX II. SPECTROCHEMICAL MAPPING USING LASER INDUCED BREAKDOWN SPECTROSCOPY AS A MORE OBJECTIVE APPROACH TO SHOOTING DISTANCE DETERMINATION ......................................................... 128 


\section{LIST OF FIGURES}

Figure 1. Left: Cross section of a cartridge set up with labeled parts. Right: Inorganic and organic chemical

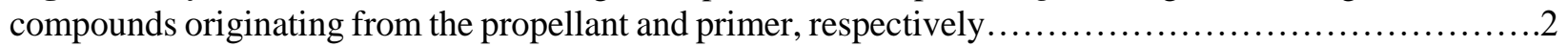

Figure 2. Penetration depth of electron beam from SEM-EDS $\ldots \ldots \ldots \ldots \ldots \ldots \ldots \ldots \ldots \ldots \ldots \ldots \ldots \ldots$

Figure 3. Diagram of change in gunshot residue distribution as the shooting distance increases from contact to close range shootings (6 inches and 12 inches) and long-range distances (24 inches and 36

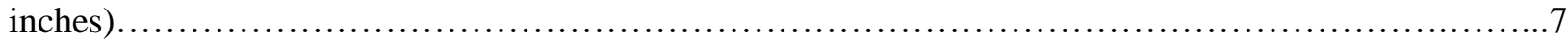

Figure 4. Chemical reaction of modified Griess chemical color test for the detection of nitrite residues and

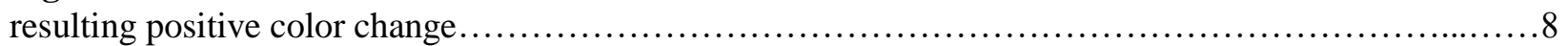

Figure 5. Chemical reaction of sodium rhodizonate chemical color test for the detection of lead residues

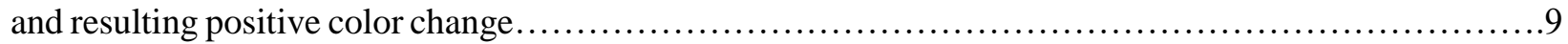

Figure 6. Layout of Following Thesis, Including Overall Goal and Specific Objectives for each

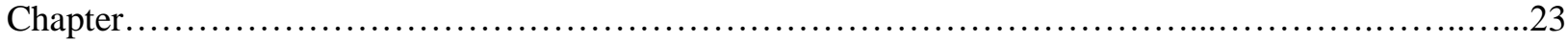

Figure 7. Experimental Design for Shooting Distance Determination by Analysis of IGSR using LIBS Including Sample Types, Variables, and Analyses Performed for Each Sample.......................25

Figure 8. LIBS experimental procedure. Custom-made ablation plate setup utilized for sample analysis (left), LIBS area of ablation for analysis (middle), and examples of spectra obtained for elements of interest (right, from top to bottom: $\mathrm{Pb}(405.8 \mathrm{~nm}, \mathrm{I}), \mathrm{Ba}(493.4 \mathrm{~nm}, \mathrm{II}), \mathrm{Sb}(259.8 \mathrm{~nm}, \mathrm{I})) \ldots \ldots \ldots \ldots \ldots \ldots \ldots \ldots .28$

Figure 9. LIBS spectra of a clothing shot at 6 inches away and background spectra of the fabric prior shooting illustrating no interferences for elements of interest from negative control fabric samples.........31

Figure 10. Results of limits of detection tests for the sodium rhodizonate assay (left), and LIBS (right), for lead (top) and barium (bottom), respectively. The inset shows a zoomed in look at Ba (493 nm) limit of detection of $0.2 \mathrm{ng}$.

Figure 11. Bar graph representing intensity patterns for $\mathrm{Pb}(405.8 \mathrm{~nm})$ for a grey fabric calibration set. From left to right, the ablation from the entrance hole begins at $0.5 \mathrm{~mm}$ and increases by $0.5 \mathrm{~mm}$ until ending at $100 \mathrm{~mm}$. The legend represents the different shooting distance used for calibration (checkered red $=$ Contact, diagonal yellow $=6$ inches, horizontal green $=12$ inches, diagonal blue $=24$ inches, solid

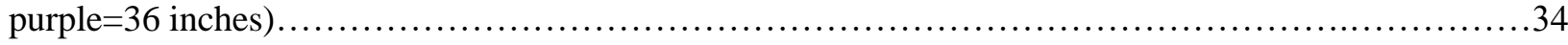

Figure 12. Heat maps of $\mathrm{Ba}(493.4 \mathrm{~nm})$ obtained from grey calibration set number 1. (a) contact; (b) 6 inches; (c) 12 inches; (d) 24 inches. .35

Figure 13. Canonical plot for grey calibration samples with grey unknowns (1,2, and 3), orange unknowns $(1,2$, and 3$)$, maroon unknowns $(1,2$, and 3$)$, navy unknowns $(1,2$, and 3$)$, and black unknowns $(1,2$, and 3$)$. All unknowns have a true shooting distance of 10 inches. (+ represents the multivariate mean for each shooting distance, outer ellipses represent $95 \%$ confidence interval for each shooting distance, and inner ellipses represent a $50 \%$ contour of observations for each shooting distance.) 
Figure 14. Canonical plot for patterned calibration samples with patterned unknowns $(1,2,3,4$, and 5), grey unknowns $(1,2,3,4$, and 5). All unknowns have a true shooting distance of 24 inches. (+ represents the multivariate mean for each shooting distance, outer ellipses represent $95 \%$ confidence interval for each shooting distance, and inner ellipses represent a 50\% contour of observations for each shooting distance.)

Figure 15. Photographs of original samples and results after the modified Griess test was performed on photographic paper (scale: $280 \mathrm{~mm} \times 150 \mathrm{~mm}$ ). Grey calibration set (from left to right: contact, 6 inches, 12 inches, 24 inches, 36 inches)

Figure 16. Photographs of original samples and results after the Mmdified Griess test was performed on photographic paper (scale: $280 \mathrm{~mm}$ x $150 \mathrm{~mm}$ ). Examples of unknowns (from left to right: orange, maroon, navy, black, and patterned).

Figure 17. Bar graph representing intensity patterns for $\mathrm{Ba}(493.4 \mathrm{~nm})$ for a blood-stained fabric calibration set. From left to right, the ablation from the entrance hole begins at $0.5 \mathrm{~mm}$ and increases by $0.5 \mathrm{~mm}$ until ending at $100 \mathrm{~mm}$. The legend represents the different shooting distance used for calibration (checkered red $=$ Contact, diagonal yellow $=6$ inches, horizontal green $=12$ inches, diagonal blue $=24$ inches, solid purple $=36$ inches $)$.

Figure 18. Heat maps of $\mathrm{Pb}(405.8 \mathrm{~nm})$ obtained from blood stained calibration set number 1. (a) contact;

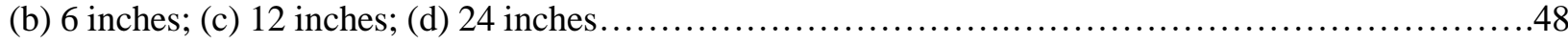

Figure 19. Canonical plot for blood stained calibration samples with blood stained unknowns $(1,2,3,4$ and 5) and white unknowns (1, 2, 3, 4, and 5). All unknowns have a true shooting distance of 6 inches. (+ represents the multivariate mean for each shooting distance, outer ellipses represent $95 \%$ confidence interval for each shooting distance, and inner ellipses represent a 50\% contour of observations for each shooting distance.)

Figure 20. Photographs of original samples (top) and results after the modified Griess test (middle) was performed on photographic paper and the Sodium rhodizonate test (bottom) was performed on the sample (scale: $280 \mathrm{~mm} \times 150 \mathrm{~mm}$ ). Blood-stained Calibration set (from left to right: contact, 6 inches, 12 inches, 24 inches, 36 inches) .53

Figure 21. Experimental design for bullet hole identification by analysis of IGSR using LIBS including substrate types, variables, and number of samples for each substrate. .59

Figure 22. Custom-made wooden structure for securing substrate samples while shooting.... .61

Figure 23. Diagram of ablation patterns created for bullet hole identification. Red square: $10 \mathrm{~mm} \times 10 \mathrm{~mm}$ inner bullet hole pattern, Blue Square: $60 \mathrm{~mm}$ x $60 \mathrm{~mm}$ outer bullet hole pattern, Green Square: $5 \mathrm{~mm} \times 5$

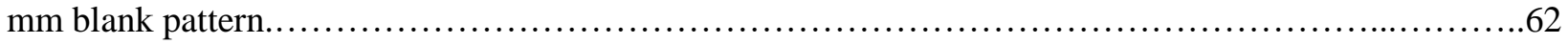

Figure 24. LIBS spectra of $\mathrm{Ba} 493.4 \mathrm{~nm}$ detected from transferred IGSR before and after application of coversheet. Top: HandiPrint Adhesive, Middle: SIRCHIE Tape Pad Adhesive, and Bottom: Whatman \#42 Paper .

Figure 25. LIBS background spectra for a) Whatman \#42 Paper b) SIRCHIE Tape Pad Adhesive and c) HandiPrint Adhesive prior shooting. No interferences present for elements of interest from negative control Whatman paper, SIRCHIE Tape Pad, and HandiPrint Adhesive samples....

Figure 26. Plywood substrate secured to custom made shooting structure using clamps. Left: plywood before shooting. Right: Plywood with visible firearm discharge residues after being shot. .68 
Figure 27. Drywall substrate secured to custom made shooting structure using clamps. Left: Drywall before shooting. Right: Drywall with visible firearm discharge residues after being shot .... .68

Figure 28. Glass substrate secured to custom made shooting structure using clamps. Left: Glass before shooting. Right: Glass with visible bullet hole after being shot.

Figure 29. LIBS background spectra for a) wood b) glass and c) drywall prior shooting. No interferences present for elements of interest from negative control wood, glass, and drywall samples...... .69

Figure 30. HandiPrint adhesive after transferring gunshot residues off of a wood substrate..............71

Figure 31. HandiPrint adhesives after transferring gunshot residues off of drywall substrates. Left: Large sections of adhesive covered in the paper coating of drywall. Right: Small areas of adhesive covered in

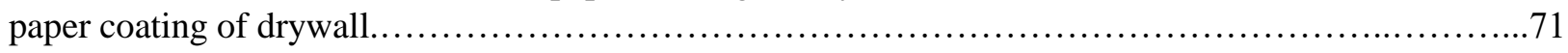

Figure 32. HandiPrint adhesive after transferring gunshot residues off of a glass substrate...............71

Figure 33. Heat maps of barium $(493.4 \mathrm{~nm})$ and lead $(405.8 \mathrm{~nm})$ for bullet hole identification, obtained set one of substrates. Left: Wood; Middle: Drywall; Right: Glass.................................73

Figure 34. Left: Photograph of a white colored textile burned with a cigarette to simulate a bullet hole. Right: Heat maps created from the spectral data obtained from the sample for Top: Ba (493.4 nm), Bottom:

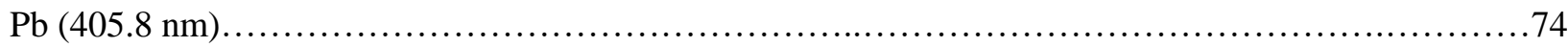

Figure 35. Experimental design for identification of ammunition composition by analysis of IGSR using LIBS including ammunition types/brands, variables, number of samples for each ammunition, and analyses performed for each sample. (IV= independent variable, $\mathrm{DV}=$ dependent variable, $\mathrm{C}=$ constant variable, $\mathrm{Q}=$

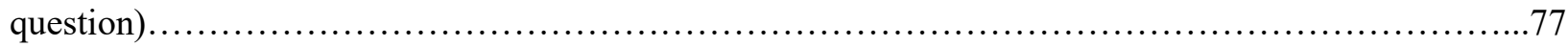

Figure 36. Microscopic photographs of gunpowder particles originating from the different standard and non-toxic ammunitions present in this study; a) Reloaded gunpowder in ammunitions using CCI, Fiocchi, TulAmmo, Remington, Winchester, and Sellier \&Bellot primers, b) gunpowder from factory-made Inceptor ammunition, c) gunpowder from factory-made Syntech ammunition, d) red polymer coating on bullet from

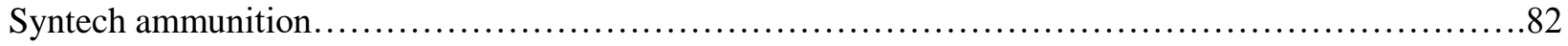

Figure 37. Photographs of original samples and results after the modified Griess test was performed on photographic paper (scale: $280 \mathrm{~mm}$ x $150 \mathrm{~mm}$ ). One set of samples shot with standard ammunitions (from

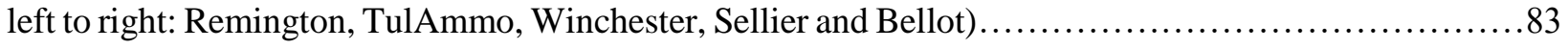

Figure 38. Photographs of original samples and results after the modified Griess test was performed on photographic paper (scale: $280 \mathrm{~mm} \times 150 \mathrm{~mm}$ ). One set of samples shot with non-toxic ammunition (from left to right: Fiocchi, CCI, Inceptor, Syntech). .84

Figure 39. SEM-EDS image of the morphological characteristics of a GSR particle originating from a Fiocchi small pistol primer and its corresponding elemental profile (elements present: K, Na, Al, Si, O)....87

Figure 40. SEM-EDS image of the morphological characteristics of a GSR particle originating from a Syntech small pistol primer and its corresponding elemental profile (elements present: $\mathrm{Ba}, \mathrm{S}, \mathrm{O}$ ).......88

Figure 41. SEM-EDS image of the morphological characteristics of a GSR particle originating from an Inceptor small pistol primer and its corresponding elemental profile (elements present: $\mathrm{Ti}, \mathrm{Zn}, \mathrm{Na}, \mathrm{O}$ )......89

Figure 42. SEM-EDS image of the morphological characteristics of a GSR particle originating from a CCI small pistol primer and its corresponding elemental profile (elements present: $\mathrm{Ti}, \mathrm{Zn}, \mathrm{Na}, \mathrm{O}$ )...........90 


\section{LIST OF TABLES}

Table 1. LIBS Parameters of Line Acquisition for Shooting Distance Determination.....................28

Table 2. Comparison of LOD's for Sodium rhodizonate test and LIBS method.......................33

Table 3. Physical measurements taken of all samples before application of color tests ( $\mathrm{P}=$ pistol,

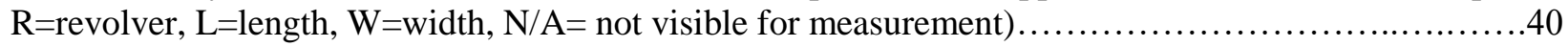

Table 4. Physical measurements taken for all samples after modified Griess and Sodium rhodizonate tests ( $\mathrm{P}=$ pistol, $\mathrm{R}=$ revolver, $\mathrm{L}=$ length, $\mathrm{W}=$ width, $\mathrm{N} / \mathrm{A}=$ not visible for measurement $) \ldots \ldots \ldots \ldots \ldots \ldots \ldots \ldots . \ldots . \ldots \ldots$

Table 5. Summary table of distance range classifications for the LIBS method, physical measurements, modified Griess and sodium rhodizonate color tests (Bold \#= model's first prediction, Non-bold \#= model's

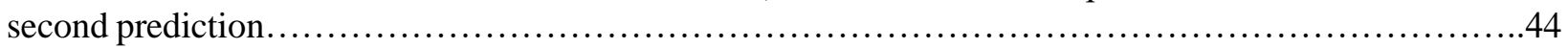

Table 6. Physical measurements taken of samples before application of color tests $(\mathrm{P}=$ pistol, $\mathrm{L}=$ length, $\mathrm{W}=$ width, $\mathrm{N} / \mathrm{A}=$ not visible for measurement).

Table 7. Physical measurements taken for samples after modified Griess and sodium rhodizonate tests ( $\mathrm{P}=$ pistol, $\mathrm{R}=$ revolver, $\mathrm{L}=$ length, $\mathrm{W}=$ width, $\mathrm{N} / \mathrm{A}=$ not visible for measurement)

Table 8. Summary table of distance range classifications for the LIBS method, physical measurements, modified Griess and sodium rhodizonate color tests (Bold \#= model's first prediction, Non-bold \#= model's

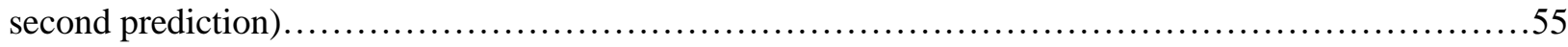

Table 9. LIBS Parameters of Inner Bullet Hole Ablation Pattern used for Bullet Hole Identification.......63

Table 10. LIBS Parameters of Outer Bullet Hole Ablation Pattern used for Bullet Hole Identification......63

Table 11. LIBS Parameters of Blank Ablation Pattern used for Bullet Hole Identification...............63

Table 12. Wavelengths Monitored for Elements of Interest in Leaded and Non-Toxic Ammunitions.......79

Table 13. Elements Detected in Standard and Non-Toxic Ammunitions by Laser Induced Breakdown

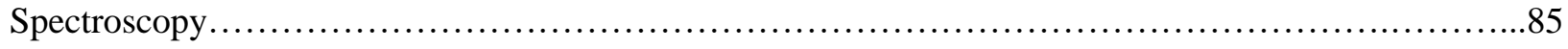

Table 14. Elements Detected in Standard Ammunitions by SEM-EDS and ICP-MS...................86

Table 15. Elements Detected in Non-Toxic Ammunitions by SEM-EDS and ICP-MS..................86

Table 16. LIBS Parameters of Spot Pattern for Non-toxic Ammunition Detection......................91

Table 17. Elements Detected in Non-Toxic Ammunitions by LIBS off SEM Stubs................................91 


\section{LITERATURE REVIEW}

\subsection{Firearm Discharge Residues and Gunshot Residues}

When a firearm is discharged, a mixture of compounds escapes from various openings in the weapon in the form of a vaporous cloud ${ }^{1}$. As the vapor cools it coalesces into fine particles that are then deposited onto the target of interest as well as surfaces surrounding the discharged firearm. These surfaces may include hands, faces, clothing and other fabrics. The residues deposited take on characteristic compositions and morphology. The inorganic components of firearm discharge residues (a.k.a. IGSR) primarily originate from the primer used and the projectile, while the organic components of the residues (a.k.a. OGSR) originate mainly from the propellant and stabilizers. Collectively, the inorganic and organic residues, along with the partially burnt and unburnt propellant, is known as firearm discharge residues (FDR).

The primer contains three major components; the initiator (e.g., lead styphnate; $\mathrm{C}_{6} \mathrm{HN}_{3} \mathrm{O}_{8} \mathrm{~Pb}$ ), the oxidizer (e.g., barium nitrate; $\mathrm{Ba}\left(\mathrm{NO}_{3}\right)_{2}$ ), and the fuel (e.g., antimony sulfide; $\mathrm{Sb}_{2} \mathrm{~S}_{3}$ ). Upon discharge, the firing pin hits the primer and ignites lead styphnate, which is also referred to as the primary explosive. Barium nitrate then begins to readily give up its oxygen to increase the temperature of the chemical reaction. Once a high temperature is reached, the antimony sulfide is ignited. This process ignites the gun powder in the cartridge case and propels the bullet through the barrel of the firearm ${ }^{2,3}$. While lead, barium, and antimony are the most common elements associated with gunshot residue (GSR), there is evidence that trace amounts of calcium, silicone, sulfur, copper, and zinc can also be linked to $\mathrm{GSR}^{4}$. The organic components of FDR have changed over time with the transition from black gun powders to smokeless powders, which are more common today. Black powders were composed mainly of potassium nitrate, sulfur, and charcoal in a 15:3:2 ratio. The smokeless powders used today have concentrations of explosives including nitrocellulose $(\mathrm{NC})$, nitroglycerine $(\mathrm{NG})$ and nitroguanidine $(\mathrm{NGu})$, stabilizers such as diphenylamine, ethyl centralite, methyl centralite, and sensitizers such as nitrotoluenes and

dinitrotoluenes ${ }^{4,5}$. Completely burnt OGSR will appear as powder, also known as soot, on a target while unburnt and partially burnt OGSR will be visible propellant particles left on a target. A diagram of a typical ammunition set up can be seen below in Figure 1. 


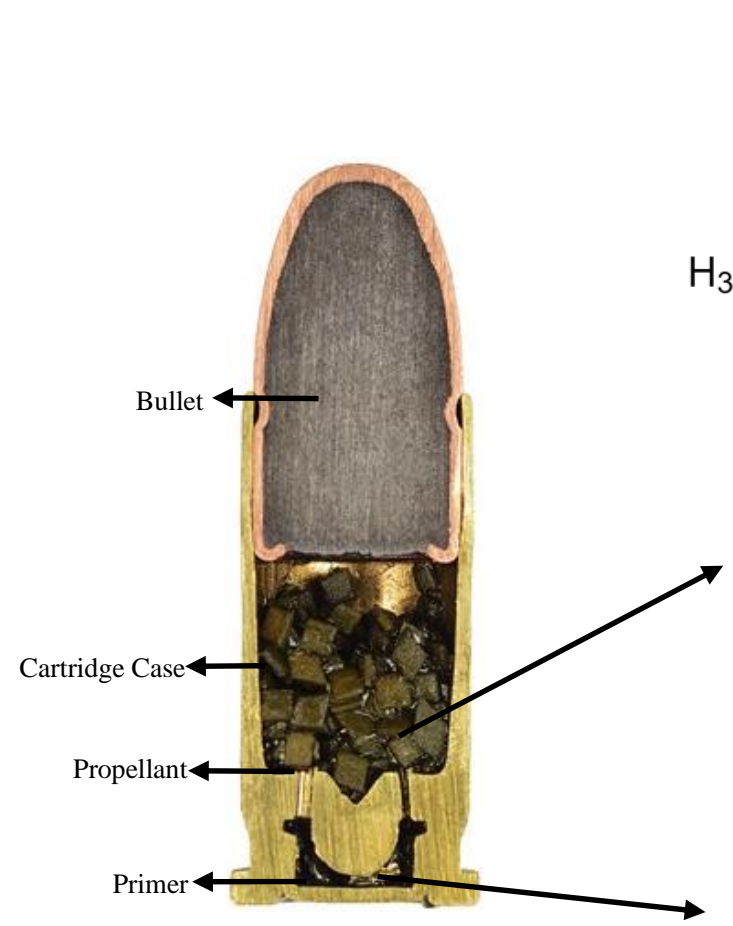<smiles>Cc1ccc([N+](=O)[O-])c([N+](=O)[O-])c1</smiles>

2,4 Dinitrotoluene<smiles>O=[N+]([O-])OCC(CO[N+](=O)[O-])O[N+](=O)[O-]</smiles>

Nitroglycerine

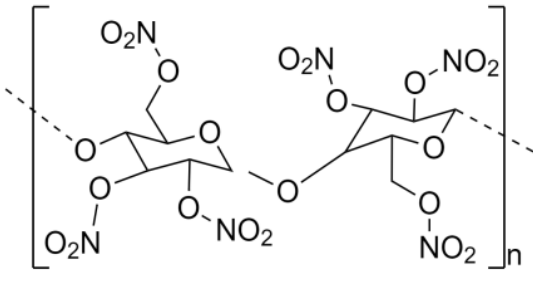

Nitrocellulose<smiles>CCN(C(=O)N(CC)c1ccccc1)c1ccccc1</smiles>

Ethyl centralite<smiles>c1ccc(Nc2ccccc2)cc1</smiles>

Diphenylamine

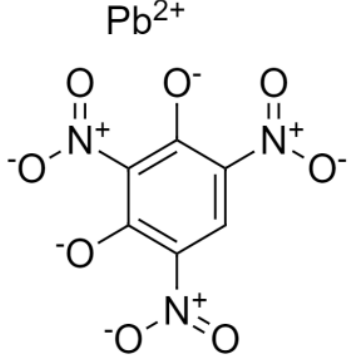

Ethyl centralite<smiles>O=[N+]([O-])O[O+][N+](=O)[O-]</smiles>

Diphenylamine

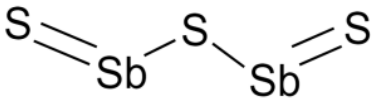

Antimony trisulfide

Figure 1. Left: Cross section of a cartridge set up with labeled parts. Right: Inorganic and organic chemical compounds originating from the propellant and primer, respectively. (Image adapted from: https://www.smithsonianmag.com) 


\subsection{Scanning Electron Microscopy-Energy Dispersive Spectroscopy Analysis}

When the vaporized inorganic components that leave the gun condense, they form characteristic spheroid shapes ${ }^{4,6}$. The most effective way of visualizing and analyzing gunshot residue particles is through Scanning Electron Microscopy-Energy Dispersive X-Ray Spectroscopy (SEM-EDX). A scanning electron microscope is capable of producing highly magnified images of microscopic specimens, such as GSR. A finely focused beam of high energy electrons scans the surface in a raster pattern to provide specific information on the size, shape, and topographical features of the particles. When the highly energized incident electron beam interacts with the specimen, different kinds of electrons are emitted; Auger electrons, backscattered electrons (BE) and secondary electrons (SE) (Figure 2). Auger electrons are released from the atom when an electron from a higher energy transfers into an inner-shell vacancy and releases energy in the form of an electron. Backscattered electrons are the electrons from the incident beam that recoil after interacting with the specimen. Secondary electrons are emitted from the specimen itself after being bombarded by the electron beam 7 . As the electron beam scans over the surface of the specimen, the excitation and relaxation of both $\mathrm{BE}$ and SE produces a high-resolution image. Areas containing elements with higher atomic numbers will be brighter than areas containing elements of lower atomic numbers. The change in brightness and shadows produces the image of the specimen on the screen. When an SEM is coupled with an electron dispersive X-ray spectrometer, the elements causing a change in brightness can be determined ${ }^{7}$. When an electron beam interacts with a specimen's surface, two types of X-rays are also emitted; characteristic X-rays and continuum X-rays. Characteristic X-rays are produced by and inelastic collision between the source proton and the atom. When a transition of an electron from a higher energy shell falls into an inner shell vacancy of an atom, the energy produced is particular to a specific atom. Continuum X-rays occur when the electrons from the electron beam interact with the nucleus of the atom. When this interaction occurs, the electrons from the incident beam loses energy and produces continuum X-rays, also known as Bremsstrahlung, which is not characteristic of the specimen atomic number. The X-rays produced are captured by a silicon lithium (Si-Li) or silicon drift (SDD) detector and displayed in a "Counts vs Energy (keV)" spectrum. 


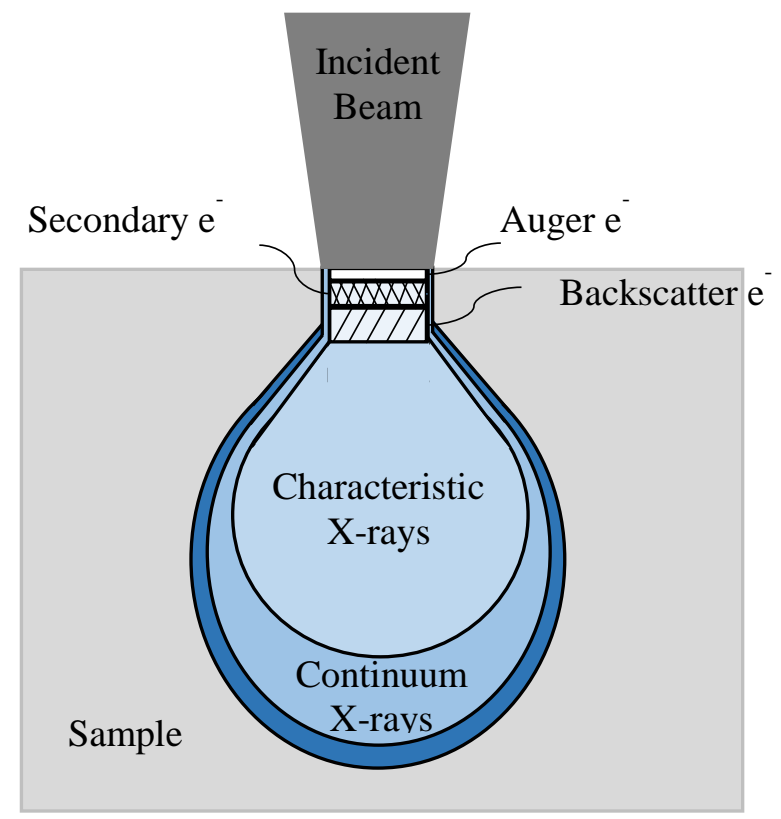

Figure 2. Penetration depth of electron beam from SEM-EDS.

Gunshot residue particles are usually between 1 and $10 \mu \mathrm{m}$ in diameter and can be separated into three spherical categories; regular spheroids, nodular spheroids, and irregular spheroids. Regular spheroids have a smooth or slightly bumpy surface, nodular spheroids occur when there is fusion between one larger and one smaller regular spheroid, and irregular spheroids have a jagged or spikey surface ${ }^{6}$. An article published by Samarendra Basu also pointed out the possibility of a "peeled orange" or "hollowed-shape" particle forming when individual smaller particles coalesce into one large particle and can reach up to $55 \mu \mathrm{m}$. The "peeled orange" particles may have a main core of barium and antimony with a surrounding flake or leaflet of lead. The "hollowed-shape" particles have one large hole with other smaller holes around the particle ${ }^{6}$. In 2018, Nunziata and Donghi revaluated the formation of Basu's "peeled orange" particles and suggested an updated model of a "strawberry" GSR particle. While Nunziata agrees with a barium/antimony core, the authors suggest that the lead around the core appears in droplets due to electrostatic forces rather than leaflets that full envelop the core of the particle ${ }^{8}$. Particles with these spheroid shapes and certain elemental compositions can be separated into three categories; characteristic of GSR, consistent with GSR, and commonly associated with GSR. The American Society for Testing and Materials (ASTM) E1588-17 separates IGSR particles in the following categories' 
1. Characteristic of GSR; particles containing the following elemental compositions:

a. Lead, barium, antimony

b. Lead, barium, calcium, silicon, tin

2. Consistent with GSR; particles containing the following elemental compositions:

a. Lead, barium, calcium, silicon

b. Barium, calcium, silicon,

c. Antimony, barium

d. Lead, antimony

e. Barium, aluminum

f. Lead, barium

3. Commonly associated with GSR; particles containing the following elemental compositions:

a. Lead

b. Antimony

c. Barium with possible sulfur presence

It is also possible to encounter a lead free or non-toxic ammunition, which would substitute the lead styphnate from the primer. The lead styphnate will be replaced with a different initiator, causing the composition of the primer, and therefore the gunshot residue composition, to change. According to ASTM E1588-17, one the following compositions are possible when analyzing lead free ammunitions ${ }^{9}$ :

1. Characteristic of non-toxic GSR

a. Gadolinium, titanium, zinc

b. Gallium, copper, tin

2. Consistent with non-toxic GSR

a. Titanium, zinc

b. Strontium

Primers used in non-toxic ammunitions contain other materials including, but not limited to: diazodinitrophenol $\left(\mathrm{C}_{6} \mathrm{H}_{2} \mathrm{~N}_{4} \mathrm{O}_{5}\right)$, tetrazene $\left(\mathrm{H}_{4} \mathrm{~N}_{4}\right)$, potassium nitrate $\left(\mathrm{KNO}_{3}\right)$, aluminium silicate $\left(\mathrm{Al}_{2} \mathrm{SiO}_{5}\right)$, and pentaerythritol tetranitrate $\left(\mathrm{C}_{5} \mathrm{H}_{8} \mathrm{~N}_{4} \mathrm{O}_{12}\right)^{10,11}$. The combination of a spheroid shape and one of these elemental groups increases the certainty of GSR being present. 
Although SEM-EDS is considered the gold standard for GSR detection on hands of individuals suspected of firing a gun, its use in bullet holes and shooting distance determinations is not common due to the prohibitive time required to scan large areas. For instance, an SEM-EDS analysis of a stub collected from a hand typically takes several hours (2-8 hours) to monitor a 1 $\mathrm{cm}^{2}$ area. As a result, screening assays such as color tests are used instead to examine larger items. Alternatively, in some instances of drive-by shootings, where IGSR are expected to deposit inside the vehicle, different surface areas of the automobile of interest can be taped with carbon stub adhesives and the cumulative residues are further analyzed by SEM-EDS. However, multiple stubs are required per vehicle and therefore collection is tedious, and the analysis is very time consuming.

\subsection{Physical Examination and Colorimetric Tests for FDR Detection}

In a criminal case involving a firearm, the shooting distance is considered the distance between the firearm muzzle and the target of interest or victim. The determination of this distance plays a crucial role in the reconstruction of crime scenes where a shooting was involved. The GSR concentration and pattern left on the target changes with the firing distance, helping to determine how the event took place. When investigating shooting distance, two common techniques used are visual examination and colorimetric tests. When doing an initial visual comparison, a set of known distance FDR patterns are compared to the sample of interest. The amount of visible powdered residue on the target can help determine if it was a close-range or long-range shooting ${ }^{12,13}$. In the case of a contact shooting, a star shaped pattern with burning, melting, or singing of the fibers surrounding the bullet hole can be seen by stereomicroscopy ${ }^{14}$. However, it has also been shown that using a silencer on a firearm may decrease the size of or eliminate the production of a star shaped bullet hole ${ }^{15}$. A shooting occurring at relative proximity is likely to show numerous unburned propellant particles in a dispersion pattern around the bullet hole, soot residues in circular forms around the bullet hole, and a large bullet hole ${ }^{16}$. On the other hand, in a long-distance shooting, it is more likely that only the bullet is able to reach the target. This results in only a small bullet hole being present on the target with less chances of seeing surrounding unburned propellant and $\operatorname{soot}^{17,18,19}$. A visual diagram of FDR distribution at different shooting distances can be seen in Figure 3. 


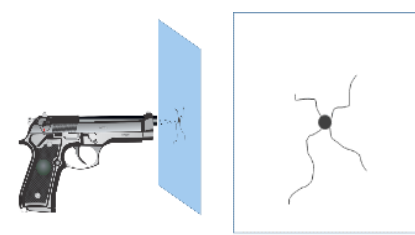

Contact

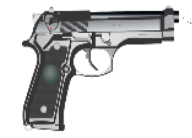

24 inches

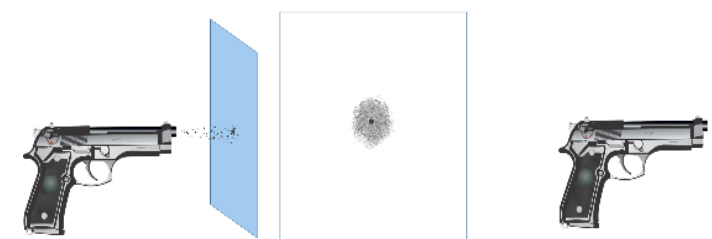

6 inches
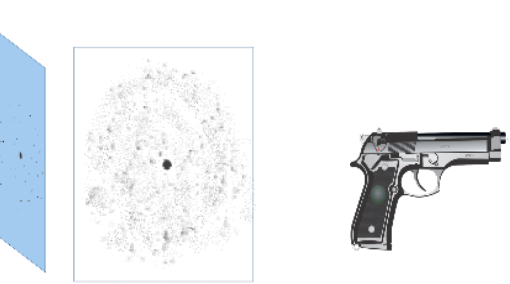

36 inches

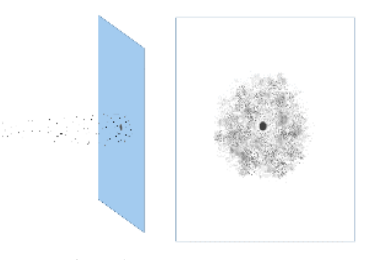

12 inches

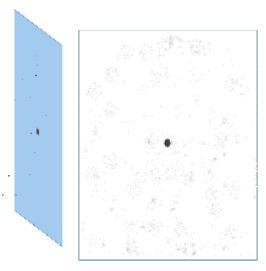

Figure 3. Diagram of changes in gunshot residue distribution as the shooting distance increases from contact to close range shootings (6 inches and 12 inches) and long-range distances ( 24 inches and 36 inches).

Chemical colorimetric tests are used to enhance the powder and gunshot residue patterns, on clothing, surrounding a suspected bullet hole. The current colorimetric tests, according the Scientific Working Group for Firearms and Toolmarks (SWGGUN), for gunshot analysis are the modified Griess test, the sodium rhodizonate test, and the Dithiooxide test ${ }^{20}$.

\subsection{Modified Griess Test}

The Griess test indicates the presence of nitrites on, for example, a victims clothing, if an orange color appears after application of the test. Nitrites will be expelled on the victims clothing when burned powder particles come in contact with the clothing. Desensitized photographic paper is soaked in a sulfanilic acid and alpha-naphthol solution and allowed to completely dry. The clothing evidence is placed entrance side down on the prepared photographic paper and the bullet hole is marked before moving forward. A piece of nitrate free cheese cloth is soaked in a diluted acetic acid solution, spread over the evidence, and heated using an iron. Once the piece of evidence is removed from the photographic paper, an orange color indicates nitrite residue. This orange color is caused when the nitrites are introduced to acetic acid and heat to form nitrous acid. The nitrous acid then forms a diazonium compound with sulfanilic acid, and finally, this compound then couples with the alpha-naphthol to produce the orange azo dye (Figure 4). Performing a Griess test allows for a visualization of the nitrite patterns which can be compared to the patterns produced by known distances for a qualitative estimation of the shooting distance ${ }^{21}$. 


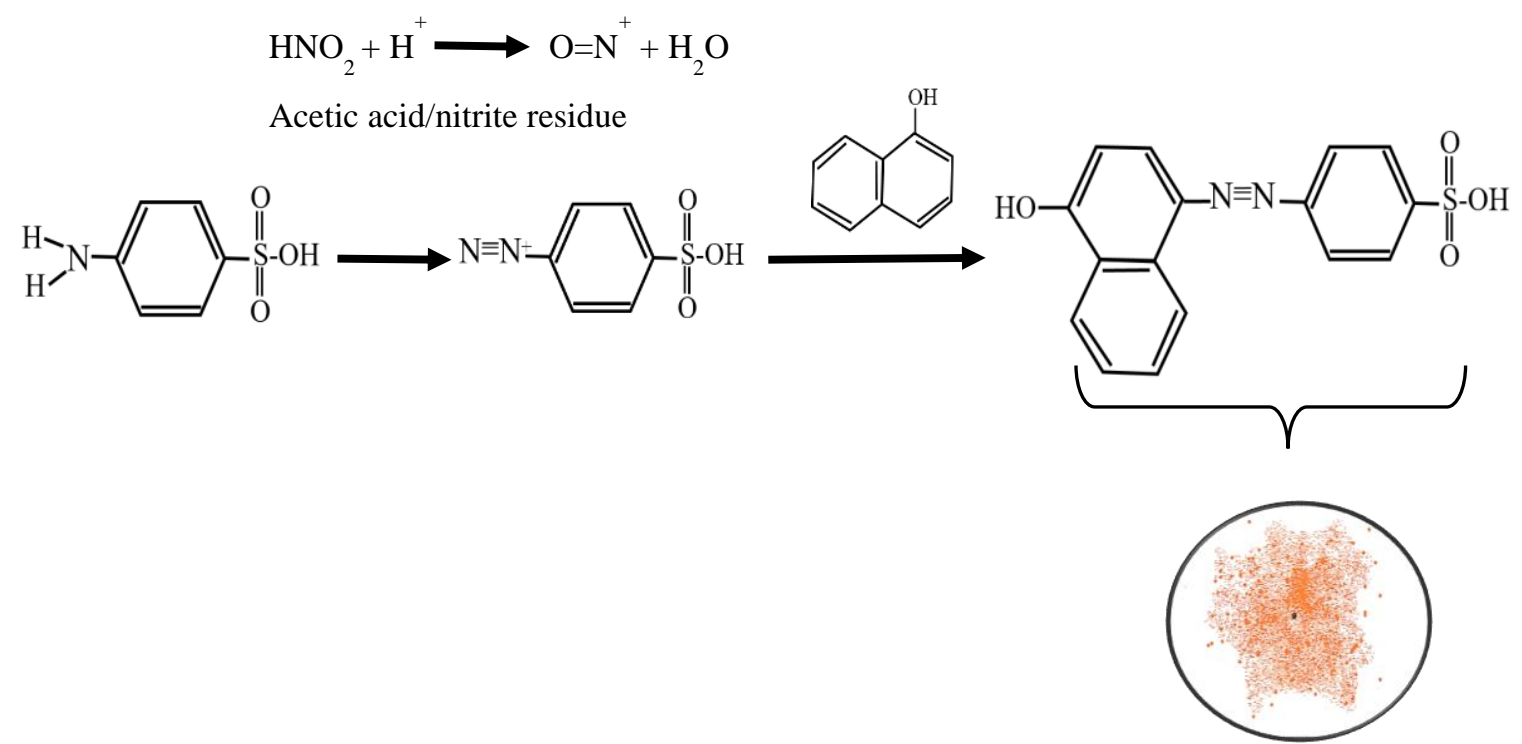

Figure 4. Chemical reaction of modified Griess Chemical color test for the detection of nitrite residues and resulting positive color change.

Further advancements in the detection of nitrites on target include Total Nitrite Pattern Visualization (TNV) which involves performing alkaline hydrolysis before performing the modified Griess test. This was first proposed in an article published by Glattstein et al. in 2000. In this study, an adhesive was placed on the target with applied pressure in order to transfer the GSR from the target to the adhesive. The adhesive was then subjected to potassium hydroxide (KOH) and placed into an oven at $100{ }^{\circ} \mathrm{C}$ for 1 hour. In the meantime, photographic paper was prepared according to the specifications in the modified Griess test. Once the adhesive was removed from the oven, the prepared photographic paper is pressed onto the adhesive at $70{ }^{\circ} \mathrm{C}$ for 1 minute using a custom-made heat press. The photographic paper is then removed to reveal the pattern. The authors of this study reported that the alkaline hydrolysis significantly increased the visualization of nitrite residues around the bullet hole, as opposed to no hydrolysis before performing the modified Griess test ${ }^{22}$.

Berger et al. recently reassessed the TNV method for practicality and use in casework. The goal of the study was to overcome the limitations of the original TNV method, including a 1-hour incubation period, custom made heating press, and the lack of testing the adhesive transfer method on bloody clothing. The authors also addressed problems common to firearm related crime scene investigations, such as long-range shooting distances and older targets. The chemical preparation of materials was the same as proposed by Glattstein et al. However, the factors used in this study included using a commercial heating press, samples shot at varying distances $(1 \mathrm{ft}, 2 \mathrm{ft}, 3 \mathrm{ft}, 4 \mathrm{ft}$, 
$5 \mathrm{ft}$, and $6 \mathrm{ft}$ ), six samples that were stored for over a year before chemical analysis, and varying heat incubation times (5 mins, 30 mins, 60 mins). The authors noted that the transfer method was limited for the blood-stained samples. They concluded that the blood prohibited the nitrite materials from adhering upon application and that direct application of the TNV method to the blood-soaked clothing produced better visualization. For long range shooting distances, the authors reported excellent visualization of nitrite patterns up to 3 feet, with fewer residues seen at 5 feet, and almost no residues seen at 6 feet. Finally, there was no significant difference in nitrite visualization between incubation times (able to lessen time from 1 hour to 5 minutes) or age of sample before analysis ${ }^{23}$.

\subsection{Sodium Rhodizonate Test}

A sodium rhodizonate colorimetric assay (Figure 5), tests for the presence of lead using a saturated

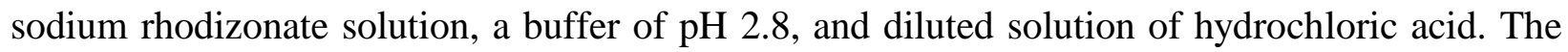
saturated sodium rhodizonate solution is sprayed over the piece of evidence, followed by the buffer solution. This will produce a pink color that may indicate the presence of lead residues. A distinctive test for lead is when a blue-violet color appears once the $\mathrm{HCl}$ solution is introduced to the evidence as well. A blue-violet color that is visible only around the bullet hole indicates a contact or close-range shot. As the shooting distance increase, the diameter of the blue-violet color should increase as well. This strong blue-violet is time sensitive and the disappearance of the color must be considered when documenting a positive result $\mathrm{t}^{24,25,26}$.

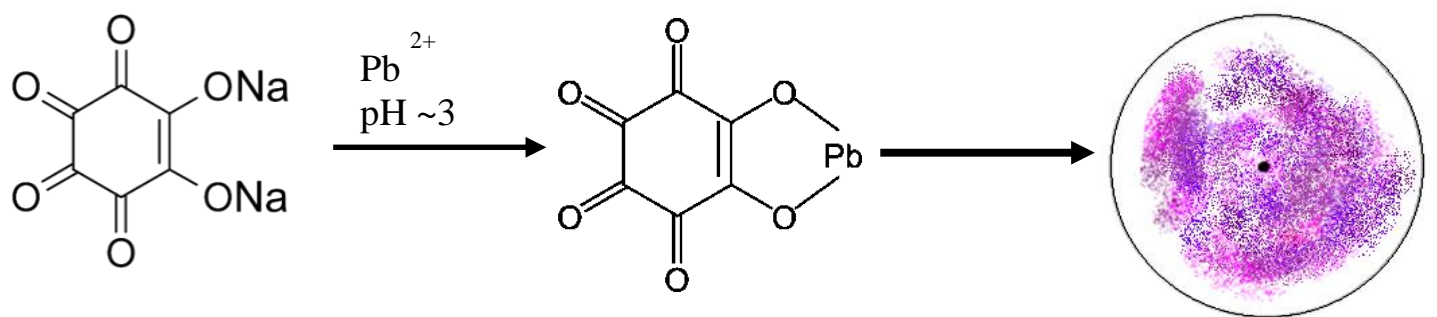

Figure 5. Chemical reaction of sodium rhodizonate Chemical color test for the detection of lead residues and resulting positive color change.

A study performed in 2011 by Andreola et al. showed that this method of GSR detection can also be performed on human skin. In the study a total of 88 histological samples from 6 deceased gunshot victims were tests using the sodium rhodizonate process both with and without the addition of $\mathrm{HCl}$. The authors reported that both methods resulted in color changes on the samples that indicated GSR, but it was noted that the additional application of $\mathrm{HCl}$ was preferred since 
sodium rhodizonate itself is not specific to GSR and reacts with hair follicles. It was also pointed out in this article that a purple color was still present 6 month after testing, but there was a significant amount of discoloration and fading 27 .

\subsection{Dithiooxide Test}

The final colorimetric test commonly used in forensics to visualize ammunition residues is the Dithiooxide (DTO) test for copper and nickel deposits around the bullet entrance hole. The DTO test is not used for primer residues, but for determining the possible composition of the bullet used in a shooting; copper, nickel. This test can also be used to differentiate between a jacketed or unjacketed bullet when combined with a lead test on the bullet hole. To perform the test, a piece of Whatman No. 1 chromatography paper is soaked in a $10 \%$ ammonium hydroxide solution and then pressed onto the bullet hole on the clothing. The paper is dried with an iron while still in contact with the clothing and then dipped into a dithiooxamide and ethanol solution to develop the color. A green color indicates the presence of copper, blue-pink color indicates nickel, and a yellow color indicates lead, which would indicate a non-jacketed bullet ${ }^{28}$. In a study performed by Lekstrom and Koons, 54 copper jacketed and semi-jacketed bullets, 24 silver-tipped (nickel coated) bullets, and 12 non-jacketed bullets were used to shoot clothing targets. Each target was then tested using the DTO process laid out above. All 54 copper bullets produced a green color, all 24 silver tipped bullets produced a pink-blue color, and all 12 non-jacketed bullets produced a yellow color, indicating the lead bullet ${ }^{28}$. However, since a Ditihooxamide test is only capable of visualizing elements located around a bullet hole, it is not used for estimating shooting distance.

While these chemical color tests are sensitive to the presence of nitrites, lead, copper, and nickel, they are not specific to gunshot residue itself. In addition, an orange or purple color change cannot be easily seen if the sample is dark in color. When performing a color test, the morphology of the GSR particles are not taken into account, only the production of a color. Therefore, even if GSR is not present, sources that contain elements within GSR will still produce a color change. These include sources in the general environment such as paint, lacquers, rubbers, paper, sunscreen, solder, eye makeup, gasoline, and textile dyes ${ }^{29}$. In addition, the sodium rhodizonate test for lead is also known to react with hair follicles, lingual papillae, and other heavy metals like barium, cadmium, tin, sulfur, and strontium ${ }^{27}$. Moreover, these tests require several chemical reagents that are hazardous (hydrochloric acid, tartaric acid, sulfanilic acid, alpha-naphthol, acetic acid, sodium 
nitrite $)^{30-35}$, are time consuming, and require multiple tests to identify the FDR components. Alternative light sources, like infrared imaging have shown promise as an additional method to help visualize FDR patterns on difficult backgrounds, such as dark colors or slightly blood-stained items. However, this technique still presents issues when the item of interest has a complex pattern or completely soaked in blood ${ }^{36-38}$.

\subsection{Current Challenges and Advances on GSR Detection}

Studies have shown that a person's occupation can lead to an accumulation of contaminants that mimic the elemental composition of GSR. For instance, one occupation with a high source of contamination is an automotive mechanic. The brake pads on a car often contain barium sulfate and antimony trisulfide to help with heat stability and lubrication of the brake pads to reduce friction $^{39}$. Due to this, the chemical makeup of brake pads has the possibility of being mistaken for GSR.

A study performed by Tucker et al. reported a total of 12 used brake pads, both OEM and aftermarket, from several cars manufactured by different companies. The 12 brakes pads were subjected to analysis by a Trace III-V+ portable XRF, to determine the elements present. All 12 brake pads contained some combination of lead, barium, and antimony. Brake rotors, as well as wheel rims, were also looked at to test the possibility of a person encountering these elements when changing a tire. These samples also tested positive for elemental compositions that were consistent with GSR. However, it was determined that when XRF is combined with SEM-EDS analysis, low false positive rates occurred due to the morphological differences between a typical GSR particle and typical brake pad particle ${ }^{39}$.

In a similar study, Ingo et al. examined the particles produced by the exothermic reaction that occurs when there is a buildup of heat and friction due to braking of a vehicle. A brand-new brake pad, manufactured for a Fiat Punto, was put through a series of intense temperature increases to simulate the heat produced during an actual breaking process. The temperature increased at a rate of $20{ }^{\circ} \mathrm{C} / \mathrm{min}$, from $25{ }^{\circ} \mathrm{C}$ to $1400{ }^{\circ} \mathrm{C}$. The residues from the heated brake pad, as well as a new brake pad, were then analyzed by SEM-EDS for chemical composition and morphology. The results showed that barium and antimony were detected in both brake pads. The only difference noted between the new brake pad and the heated brake pad was the size and morphology of the 
particles seen under SEM. The results in this study support the possibility of encountering automotive residue interferences when performing color tests on a victim's clothing ${ }^{40}$.

Another possible source of contamination is pyrotechnic material, such as flares, fireworks, and military devices. It has been found that the particles formed from the heating, burning, and explosive nature of pyrotechnic materials share similar morphological characteristics to GSR particles formed from discharging a firearm ${ }^{41-43}$. The morphology of the particles, as well as the composition of the particles, was evaluated in an article published by Mosher et al. In this study, 4 hand samples (2 left/2right) were taken from two firework technicians and 10 samples were taken from similar fireworks. The technicians' hands were sampled after putting on the display and then cleaning up after the display. For the firework samples, 5 types were sampled before combustion and then the same 5 types were sampled after combustion. To collect the samples after combustion, a SEM stub was held under the firework as it was burning. From the technicians, particles were found that had rounded or rounded-globular appearances and elemental compositions that were consistent with GSR $(\mathrm{Pb} / \mathrm{Ba}, \mathrm{Ba} / \mathrm{Sb}, \mathrm{Pb} / \mathrm{Sb})$. The samples taken directly from the fireworks (combusted and un-combusted) all showed particles with compositions consistent with GSR particles $(\mathrm{Pb} / \mathrm{Ba}, \mathrm{Ba} / \mathrm{Sb}, \mathrm{Pb} / \mathrm{Sb}$ ). However, the particles did not share similar morphologies to GSR particles. The article described the pyrotechnic particles "elongated globular" which are not representative of typical GSR ${ }^{44}$.

The possibility of fireworks as a source of GSR contamination was also assessed by Grima et al. For the study, SEM stubs were placed near the ignition site of a professional fireworks show. The stubs collected the pyrotechnic particles that fell from the fireworks after they were combusted. Unlike the previous article, during a different firework display SEM stubs were placed $500 \mathrm{ft}$ away from the ignition site to see how the firework particles traveled after combustion. This would help to gauge the possibility of the general public being contaminated with the pyrotechnic particles. A sample was also taken from the technician's hair using a swab soaked in isopropyl alcohol. The results showed that $\mathrm{Pb}, \mathrm{Ba}, \mathrm{Sb}$, and other elements found in GSR, were found on the SEM collection stubs as well as trace amounts found on the swab taken from the technician's hair. It is important to note that the SEM stubs placed among the general public during the fireworks display also contained $\mathrm{Pb}, \mathrm{Ba}$, and $\mathrm{Sb}$. This shows that it is very possible for people's clothing to be 
exposed to contaminants from firework material that would interfere with the color produced by a chemical color test ${ }^{45}$.

Furthermore, in 2009, Berk tested several airbag residue standards from several automobile manufacturers including Volkswagen, Chevorlet, Dodge, Mercury, Ford, Buick, Pontiac, and Jeep. These manufacturers use percussion primers in their airbags as part of the chemistry that deploys the system upon impact. After assessing the particles produced by the air bags, it was concluded that all of the airbag standards had at least 2 characteristic GSR particles (consisting of lead, barium, antimony with a spherical shape). However, the author asked GSR specialists to also classify all particles that were categorized by SEM-EDS as GSR. While the detected particles had the three characteristic GSR elements (lead, barium, antimony) and the morphology of a GSR particle, the analysts were able to classify most of the suspected particles as not being GSR. This was due to the presence of other elements that are not typically found in gunshot residues or firearm primers, including elevated levels of cobalt and aluminum, and zirconium/phosphorous peaks ${ }^{46}$.

A more extensive sampling population was tested by Garofano et al. The methods included using 175 SEM stubs to take samples from the hands of individuals working in 25 different occupational fields. After analysis by EDS, the occupations that tested positive for elemental compositions representative of GSR were car radio installers, those working with cartridge operated industrial tools (nail guns), automobile electricians, motor repair auto mechanics, and gas station attendants. In agreement with the studies previously mentioned, Garofano et al. also found brake repair auto mechanics and pyrotechnic/fireworks experts to test positive for the presence of $\mathrm{Pb}, \mathrm{Ba}$, and $\mathrm{Sb}^{47}$. As discussed above, possible contaminants can be differentiated from GSR by implementing additional instrumental methods for analysis. Therefore, different analytical techniques have been studied to detect gunshot residues and to estimate shooting distance, including Fourier Transform Infrared Spectroscopy (FTIR), Raman Spectroscopy, Atomic Absorption Spectrometry (AA), Atomic Force Microscopy (AFM), and X-ray Fluorescence Spectrometry (XRF).

In 2013 Bueno et al. attempted to determine ammunition caliber based on firearm discharge residues and their detection using ATR-FTIR. To collect samples, clothing items were shot from a distance of $30 \mathrm{~cm}$ using 3 different caliber firearms; a 0.40 -inch caliber, $9 \mathrm{~mm}$ caliber, and a 0.38 -inch caliber. Individual gunpowder particles, totaling 160 particles, were recovered from each ammunition and subjected to ATR-FTIR analysis. This was considered the validation set of 
gunpowder particles. The FTIR spectrum collected from each particle was an average of 20 individual scans. An additional 15 gunpowder particles were collected and acted as "blind" samples for classification. Data collected from the spectral region of $1800-600 \mathrm{~cm}^{-1}$ was used to perform cross-validated pairwise discriminant analysis on the validation set and "blind" gunpowder particles. The spectral difference between calibers resulted in an overall correct classification accuracy of $93.5 \%$ for the 0.38 -inch caliber and $100 \%$ for both 0.40 -inch caliber and the $9 \mathrm{~mm}$ caliber weapon. While this method is focused on gunpowder particles, rather than gunshot residues it illustrates discriminant analysis as a classification method for gunshot residue analysis. ${ }^{48}$

Sharma and Lahiri investigated the possibility of analyzing organic nitroglycerine by FTIR for the purpose of shooting distance determination. In preparation, white cotton clothes were shot using a rifle at distances of 3 in, $6 \mathrm{in}, 9 \mathrm{in}$, and $12 \mathrm{in}$. For analysis, four $2 \mathrm{~cm} \mathrm{x} 1 \mathrm{~cm}$ sections were cut from each of the samples. The first section was cut $3 \mathrm{~cm}$ away from the center of the bullet hole, and the 3 subsequent sections were $5 \mathrm{~cm}, 7 \mathrm{~cm}$, and $9 \mathrm{~cm}$ away f.rom the bullet hole. The OGSR from the sections was then extracted using acetone. The OGSR present after the acetone dried were used to make a KBr pellet that was then analyzed by FTIR. The authors then created a plot of the relative transmittance of nitroglycerine with respect to the distance from the bullet hole. This was done for each shooting distance to compare the relative transfer of nitroglycerine between the samples. It was found that the transmittance observed for nitroglycerine decreased as the shooting distance increased for each 3 inch increment ${ }^{49}$. The major disadvantage of the sampling method employed in this study is the destruction of the sample.

The Lednev research group has made several advancements toward the use of Raman spectroscopy to detect both organic and inorganic components of gunshot residues ${ }^{50,51,52}$. In 2012, a research project headed by Justin Bueno explored the potential of Raman spectroscopy to differentiate between two different caliber firearms based on the produced GSR. Using partial least squares statistical classification method, the research group was able to differentiate between CCI $9 \mathrm{~mm}$ Luger and Winchester .38 special ammunition. Cloth targets were shot from $0.3 \mathrm{~m}$ away and a total of 8 samples shots were completed for each ammunition. Following shooting, unburned propellant particles were transferred from the target and adhered to an aluminum slide for Raman analysis. A total of 78 particles were analyzed with 4 Raman spectra being collected from each 
particle. The ammunitions had identical peaks at $983 \mathrm{~cm}^{-1}$ and $692 \mathrm{~cm}^{-1}$, which were indicative of lead styphnate and barium sulfate residues. This study showed that Raman spectroscopy is a promising technique for detecting the different inorganic and organic components in ammunitions of different calibers and charge loads 50 .

Furthermore, Bueno and Lednev evaluated the potential of Raman microscopectroscopic mapping as a way of detecting inorganic and organic gunshot residues. In this study, three cotton substrates were shot from $0.3 \mathrm{~m}$ away using 0.38 special ammunition and a 0.38 caliber firearm to form a training set. To collect GSR samples from the cotton substrate, double-sided adhesive tape was attached to a microscope slide (covered in aluminum foil) and pressed onto the cotton to obtain particles. The adhesive was then subjected to Raman spectroscopy using a $780 \mathrm{~nm}$ Raman excitation laser. Spectra were collected from $3,300 \mathrm{~cm}^{-1}$ to $50 \mathrm{~cm}^{-1}$, but only the fingerprint region was of interest $\left(1,850 \mathrm{~cm}^{-1}\right.$ to $\left.320 \mathrm{~cm}^{-1}\right)$. From the training set, three tape swatches, three OGSR particles, and five IGSR particles were mapped, totaling 785 spectra collected. Once the training set was complete, a tape lift was taken from an independent cotton sample and subjected to Raman spectroscopy using the same mapping technique performed for the training set. A total of 650 Raman spectra were collected from the mapping and it was determined that the proposed method was capable of detecting and plotting potential GSR particles in 3D, within hours. When performing partial least squares-discriminant analysis on the data collected from the unknown tape lift, the authors reported $85.0 \%$ and $99.1 \%$ for true positive and true negative rates, respectively, for OGSR and $90.4 \%$ and $92.9 \%$ true positive and true negative rates, respectively, for IGSR. However, the authors noted that in order for a GSR to be correctly classified using this method, it should be atleast $3.4 \mu \mathrm{m}$ in size ${ }^{53}$. The Lednev research group went on to expand this study in 2018, using a larger sample size for validation. Additionally, they included samples that could potentially provide false-positive assignments including tape lifts from the hands of automotive mechanics. The results showed that none of the spectra collected from the mechanics were misclassified as OGSR. However, two spectra were misclassified as IGSR due to the presence of the heavy metals associated with both IGSR and automotive tires ${ }^{54}$.

Atomic force microscopy was evaluated as an instrumental technique for the estimation of shooting distance by Mou et al. To obtain samples, 8.5 in x 11 in polyethylene sheets were shot using a long-rifle at 6 inches, 2 feet, 10 feet, and 20 feet. Polyethylene was chosen as the target 
because of its transparency and compatibility with AFM. A $28.3 \mathrm{~mm}^{2}$ area centered around the bullet hole was then scanned using the tapping mode of an atomic force microscope. The AFM was used for quantitative measurements pertaining to the particles present; size and density around the bullet hole. After analyzing all 4 shooting distances, it was concluded that as the shooting distance increased, the relative size of the particles present, around the bullet hole, decreased. A decrease in the particle density count in the $28.3 \mathrm{~mm}^{2}$ area scanned was also seen to decrease with the increase of shooting distance ${ }^{55}$. Although AFM showed to the effective in the distance estimation, the limitations of this approach includes the lack of chemical confirmation of the particle, time of analysis (hours for scanning and moving sample for full analysis), complexity and cost of the instrumentation.

The use of AA for determining shooting distance was reported by Candela et al. The study focused on the analysis of lead on samples of Whatman no. 1 paper after being shot at from $5 \mathrm{~cm}, 10 \mathrm{~cm}$, $20 \mathrm{~cm}, 25 \mathrm{~cm}, 30 \mathrm{~cm}, 35 \mathrm{~cm}, 40 \mathrm{~cm}, 45 \mathrm{~cm}, 50 \mathrm{~cm}, 60 \mathrm{~cm}, 80 \mathrm{~cm}$, and $100 \mathrm{~cm}$. Three circles, having diameters of $1.4 \mathrm{~cm}, 5 \mathrm{~cm}$, and $10.2 \mathrm{~cm}$, were then cut from the Whatman paper and heated in a solution of hydrochloric acid. The amount lead present in the hydrochloric acid solution was then analyzed by AA. It was found that there was a general decrease in the amount of lead present $(\mu \mathrm{g})$ and the shooting distance increased. A linear relationship between the shooting distance and the amount of lead present, for each circular section samples, was also found. The linear relationships were used as calibration equations to be used in further casework ${ }^{56}$. One advantage of AA is its high sensitivity and selectivity; however, the method is destructive of the sample and time consuming (hours to perform full analysis).

Another instrumental technique suggested for distance determination was milli-X-ray fluorescence. In a study, clothing samples composed of denim, polyester/acrylic, and stain were shot at from various distances ranging from $10 \mathrm{~cm}$ to $150 \mathrm{~cm}$. The samples were then stretched tight on the sample holder and subjected to m-XRF analysis. Heat maps were created from the intensities detected for different elements $(\mathrm{Pb}, \mathrm{Ti}, \mathrm{Gd}, \mathrm{Zn})$ to visualize the GSR patterns. Linear graphs presenting the relationship between the intensity of lead and the distance from the entrance hole were also made for all tested distances. The heat maps showed a decrease in elemental intensities of lead as the shooting distance from the target increased ${ }^{57}$. This method provides the unique advantage of creating chemical mappings that enhance objectivity and selectivity of GSR 
patterns around the entrance hole, however the technique is not available in the market, has a small working distance of only $2 \mathrm{~mm}$, and the reproducibility of the measurements is highly influenced by the flatness of the sample ${ }^{58}$.

\subsection{Laser Induced Breakdown Spectroscopy}

In general, limitations that exist with the previous instrumental techniques are bulk analysis with no or limited spatial information, destruction of sample, no simultaneous detection of elemental composition or low sensitivity for certain elements, high cost and complexity, and lack of portability or practicality. One method that can overcome these major limitations is Laser Induced Breakdown Spectroscopy (LIBS). A LIBS instrument uses a highly powered, extremely focused, pulsing laser to interact with a sample. As the laser comes in contact with the sample, a temporal plasma is created on the surface. The extreme heat of the plasma causes species in the sample to vaporize, resulting in free, excited atomic and ionic forms of the elements contained with the sample. As the excited states relax, light is emitted at wavelengths specific to the elements present. The light is captured by a fiber optic cable and received by a spectrometer. Two common spectrometers used during LIBS analysis are the Czerny Turner spectrometer and the Echelle spectrometer. A Czerny Turner spectrometer utilizes a diffraction grating and two mirrors. When the light moves through the entrance slit it bounces of a mirror to collimate the light, goes through a diffraction grating to separate the light into individual wavelengths, bounces off another mirror to focus similar wavelengths, and then enters into a detector. On the other hand, the Echelle spectrometer uses two dispersion elements, a grating and a prism. When light enters an echelle

spectrometer it bounces off a collimating mirror, goes through a diffraction grating to separate wavelengths, goes through a prism that is perpendicular to the diffraction grating so overlapping wavelengths are further separated, and then bounces off another mirror before entering the detector $^{59}$. Two common detectors that receive the light from the spectrometer are known as coupled-charge device (CCD) and intensified coupled-charge device (ICCD). The detectors are responsible for converting the information contained within the emitted light into digital spectra and data. As the photons interact with the detector, charges are generated and then converted into digital representations of the pattern of light on the detector camera. Compared to a CCD detector, an ICCD detector contains a microchannel plate which prevents light from reaching the detector when turned off. When the microchannel plate is turned on, the light passing through the plate is 
amplified before reaching the detector. Both detectors supply an output of data in the form of an intensity vs. wavelength spectrum ${ }^{59}$.

LIBS instruments are commonly equipped with Nd:YAG lasers that have fundamental wavelengths of $1064 \mathrm{~nm}, 532 \mathrm{~nm}, 355 \mathrm{~nm}, 266 \mathrm{~nm}$, and $213 \mathrm{~nm}$. The energy of the Nd:YAG can vary between $15 \mathrm{~mJ}$ and $100 \mathrm{~mJ}$ depending on the specific instrument. There are several parameters than can be considered in the method development, including the gate delay, the spot size, the pulse repetition rate, the pulse energy, and stage velocity. A gate delay is a set amount of time, usually microseconds, before the detector starts collecting data. The optimization of the gate delay helps to optimize the signal to noise ratio, as the background emission decreases more rapidly than atomic and ionic emissions. A spot size is the diameter of the laser beam when focused on the sample; it can be commonly modified from $4 \mathrm{um}$ to $250 \mathrm{um}$. The pulse repetition rate is the frequency of the laser and the pulse energy is the percentage of laser output energy. These parameters, along with beam size, are critical in producing the irradiance needed to induce the microplasma and optimal temperature to excite species of interest. Finally, the stage velocity is the speed at which the sample stage in the ablation chamber will move and therefore controls how much material will be ablated ${ }^{60}$.

Laser induced breakdown spectroscopy (LIBS) has recently shown promise as a rapid, simple, and versatile detection method for gunshot residues ${ }^{2,61-68}$.

In 2007, Dockery and Goode introduced the application of LIBS for determining the presence of gunshot residues on an individual's hands. To generate samples, a single shot was fired from a clean weapon six times, sampling the individual between every shot. An additional generation required an individual to fire 5 consecutive shots, 6 different times, and sampling from the individual between each set of five shots. Custom made GSR stubs were used to collect from the trigger finger, webbing between the thumb and forefinger, and back of the thumb of an individual who discharged the firearm. The samples were analyzed by LIBS using a single shot in 20 different locations on the stub. To avoid decreasing the signal to noise ratio of the spectra, the spectrum with the largest observed signal was chosen to represent one stub. Seven different emission lines indicative of barium were observed as positive test results for GSR. The study found that barium was detectable in all 7 observed emission lines when the collection occurred between the sets of 5 shots. On the other hand, in between single shots barium was detected in 2 observed emission 
lines, on average. The methodology in this study was not fully optimized, however, it did show the potential of LIBS as a screening method for the detection of gunshot residues from an individual's hands. ${ }^{2}$

Dockery's research group further applied this methodology in 2008, with Rosenberg, to evaluate the lifetime of detectable gunshot residues on the hands of an individual who fired a weapon. Three volunteers fired a revolver six times to generate gunshot residue particles on their hands. Collection of GSR then occurred at eleven different time intervals after shooting; 12 hours, 24 hours, 36 hours, 48 hours, 60 hours, 72 hours, 96 hours, 120 hours, 144 hours, 168 hours, and 192 hours. Participants were asked to proceed with normal activities during the time between discharge of the weapon and collection. Similar to the previous study, a detectable intensity of barium (455.4 nm) was considered positive for gunshot residue. The authors concluded that gunshot residue particles were still detectable on an individual's hands 5 days after the shooting incident occurred, which is atypical of the optimal collection time for GSR. ${ }^{63}$

In 2009, Silva et al. performed a similar detection experiment with an extended sample size. Nine participants were asked to discharge a firearm between 1 and 5 times to deposit a sufficient amount of gunshot residues on their hand. A total of 97 gunshot residues samples were collected and analyzed by laser-induced breakdown spectroscopy. Unlike Dockery's research group, Silva et al. observed emission lines characteristic of lead, barium, and antimony, as suggested by the NIST library at the time of the study. The integrated data for lead, lead, barium, and antimony obtained from shooter and non-shooter samples were subjected to statistical analyses including principlal component analysis and SIMCA to assess the distinguishability between shooters and nonshooters. All 97 samples collected from individuals who fired a gun were correctly classified as "shooter" samples and no false positive results were seen for samples collected from individuals who did not fire a gun. ${ }^{62}$

The simultaneous detection and characterization of inorganic and organic gunshot residues using gas chromatography-mass spectrometry (GC-MS) and LIBS, respectively, was investigated by Tarifa et al. in 2015. Forty-two swabs (double-sided) were collected from the hands of 9 police officers immediately after discharging a firearm loaded with $9 \mathrm{~mm}$ Luger cartridges. One side of the swab was placed into a glass vial to be analyzed by head-space GC-MS, and the other side of the swab was kept in a centrifuge tube. The swab in the centrifuge tube underwent a liquid 
extraction process and the resultant solution was deposited onto a Teflon stub and allowed to dry before analysis by LIBS. The organic materials being monitored were ethyl centralite, nitroglycerine, 2,4-dinitrotoluene, and diphenylamine. Out of the nine officers that were sampled, 5 of them tested positive for high levels of both nitroguanidine and diphenylamine (5-7 ng), 2 of them tested positive for both components but in lower quantities $(\sim 1 \mathrm{ng})$, and 2 of them tested negative for both components. The authors used an extensive elemental profile, as suggested by the ASTM standard, when analyzing the IGSR (e.g., Al, Ba, Ca, Co, Cr, Cu, Fe, K, Li, Mg, Mn, $\mathrm{Na}, \mathrm{Ni}, \mathrm{P}, \mathrm{Pb}, \mathrm{S}, \mathrm{Sb}, \mathrm{Si}, \mathrm{Sn}, \mathrm{Sr}, \mathrm{Ti}, \mathrm{Zn}, \mathrm{Zr}$ ). However, most of the elements were eliminated due to interferences from the cotton swabs and background hand samples for additional police officers (e.g., Al, Ba, Ca, Cr, Cu, Fe, K, Li, Mg, Mn, Na, Ni, P, Pb, S, Si, Sr, Ti, Zn). After analyzing 42 swabs from 9 different police officers, the authors found that 6 out of the 9 participants tested positive for having both $\mathrm{Pb}$ and $\mathrm{Ba}$ present on their hands. However, the authors also point out that $\mathrm{Sb}$ was not detectable on any samples due to its low concentration and interfering Fe emission lines. $^{66}$

Many advantages exist with the use of LIBS for shooting distance determination by the analysis of gunshot residue. Sample preparation and analysis are both simple and fast. LIBS has the capability of performing direct ablations on a solid material with no sample preparation. During direct analysis, minimal damage is done to the sample which allows for subsequent analysis by other methods, if necessary. LIBS also has superior sensitivity and selectivity capabilities than color tests. Unlike colorimetric assays, LIBS is able to detect multiple elements simultaneously. The elements detected are also confirmed by multiple atomic and ionic emission lines, which increases the certainty of its presence while decreasing the rate of false positives. LIBS also has the ability of detecting common GSR elements in the low ppm concentrations, even those present in lead-free and modern ammunition ${ }^{59}$. The unique advantage of multi-elemental capability and spatial resolution also helps to rule out potential interferences. LIBS instruments are very versatile and can be operated at the laboratory, mobile labs and in the field. Moreover, it is cost-effective and easy to operate. These benefits make LIBS a promising technique for analyzing gunshot residues on clothing samples and other substrates.

In a recent study performed by Lopez-Lopez et al., the ability of LIBS to visualize gunshot residue patterns was demonstrated. To obtain samples, white cotton cloths were shot at from distances of 
$30 \mathrm{~cm}, 50 \mathrm{~cm}$, and 150 centimeters. To recover the GSR from the samples a double-sided adhesive was applied to the cloth samples and then attached to an aluminum sheet. When the adhesive-sheet was inserted into the ablation chamber, a $130 \mathrm{~mm}$ x $165 \mathrm{~mm}$ area, centered around the bullet hole, was chosen for analysis. This area resulted in 31,050 individual shots performed by the laser, and therefore, 31,050 individual LIBS spectra. Heat maps were created for $\mathrm{Sb}, \mathrm{Ba}$, and $\mathrm{Pb}$ from the intensities detected for the elements at each shooting distance by LIBS. The heat maps produced were able to represent the distribution pattern around the bullet hole, with the bullet hole be a circular region of high intensity. The results ultimately showed a decrease in the elemental intensities detected by LIBS as the shooting distance was increased. Although this study did not use samples shot at unknown distances to test the capability of this method for distance determination, it proved that LIBS is able to accurately depict gunshot residue patterns on clothing samples ${ }^{69}$. 


\section{OBJECTIVES OF OVERALL PROJECT}

Current methods for shooting distance determination and identification of potential entrance holes focus on the use of chemical color tests and physical characteristics. However, these tests have major drawbacks, including selectivity and sensitivity issues. While SEM-EDS analysis is good for small samples, it is excessively time consuming for larger sampling areas. Therefore, there is a critical need to enhance detection methods for FDR on clothing and other target materials. The following thesis focused on implementing the method of Laser Induced Breakdown Spectroscopy for gunshot residue analysis and shooting distance determination. The methods proposed kept the integrity of the sample intact, allowing for sample reanalysis, if needed. This can become an important aspect in a court of law when reanalysis is requested. While the study performed by Lopez-Lopez et al. only evaluated the use of LIBS on white cotton clothes, the following research evaluated other clothing samples that are common to crime scenes; dark clothing, patterned clothing, and blood-stained clothing. The experimental design tested the capability of LIBS to accurately detect GSR intensities despite the change in background appearance. Different manufacturers of primers were also used throughout the study, as to increase the population of primers potentially encountered at a crime scene. Chemical mapping methods, linear graphs, and multivariate analysis methods were performed on the obtained data to give better visual representation of the GSR present. Figure 6 below give a layout of the following Thesis with specific goals and objectives. 


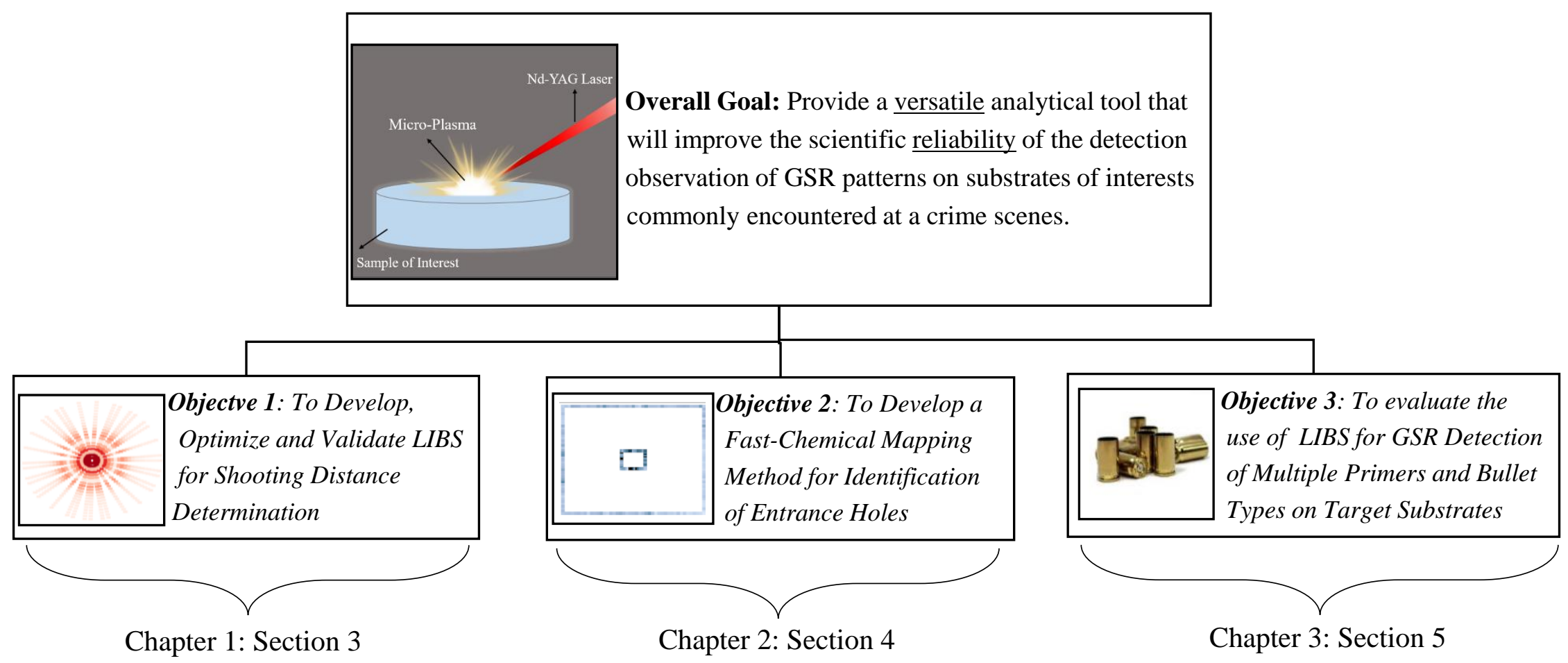

Figure 6. Layout of following thesis, including overall goal and specific objectives for each chapter. 


\section{CHAPTER 1: THE DEVELOPMENT, OPTIMIZATION, AND VALIDATION OF LIBS FOR SHOOTING DISTANCE DETERMINATION}

\subsection{Overview of Project}

This chapter proposes the application of Laser-Induced Breakdown Spectroscopy (LIBS) as a novel and practical method to estimate shooting distances in firearm-related criminal events. Clothing samples fired at different intervals were analyzed by LIBS, and the spectral data collected from these experiments were used to construct 2D elemental maps. Spatial distributions of inorganic gunshot residues (IGSR), such as lead, barium, and antimony, were used to classify the shooting range. Ninety-eight cotton textiles of different colors, fabric patterns, and condition were shot using a $9 \mathrm{~mm}$ pistol and a Rossi revolver with different ammunition. Sixty of the fabrics were shot at known distances to create control training sets, while the remaining thirty-eight samples were used as blind items, of unknown distances to the examiner, to test the accuracy of the method. The performance of the LIBS method was compared to visual and colorimetric tests currently used by crime laboratories. Results show that LIBS offers superior sensitivity, selectivity, reproducibility, and accuracy than color tests. Unlike the conventional assays, LIBS produce permanent chemical images that allow objective statistical treatment of the data. Principal Component Analysis and Discriminant Analysis of LIBS data resulted in $100 \%$ correct classification of the shooting ranges, while color tests resulted in 58.5\% correct classification, 10\% misclassification, and $31.5 \%$ inconclusive results. The LIBS method offered improvements over conventional tests such as simplicity, versatility, and reliability.

The following chapter is divided into two sections that discuss clothing samples commonly submitted as evidence from crime scene investigations. Section 3.2. is an adaption of the article "Spectrochemical Mapping Using Laser Induced Breakdown Spectroscopy as a More Objective Approach to Shooting Distance Determination" published in the Journal Spectrochimica Acta Part

B: Atomic Spectroscopy, where the use of LIBS for shooting distance determination on dark colored and patterned clothing samples was assessed. Within section 3.3., the same shooting distance determination method using LIBS was tested on bloody clothing to represent real samples found at crime scenes. Figure 7 gives a detailed layout of the objectives, number of samples, and type of analyses performed for each sample set. 


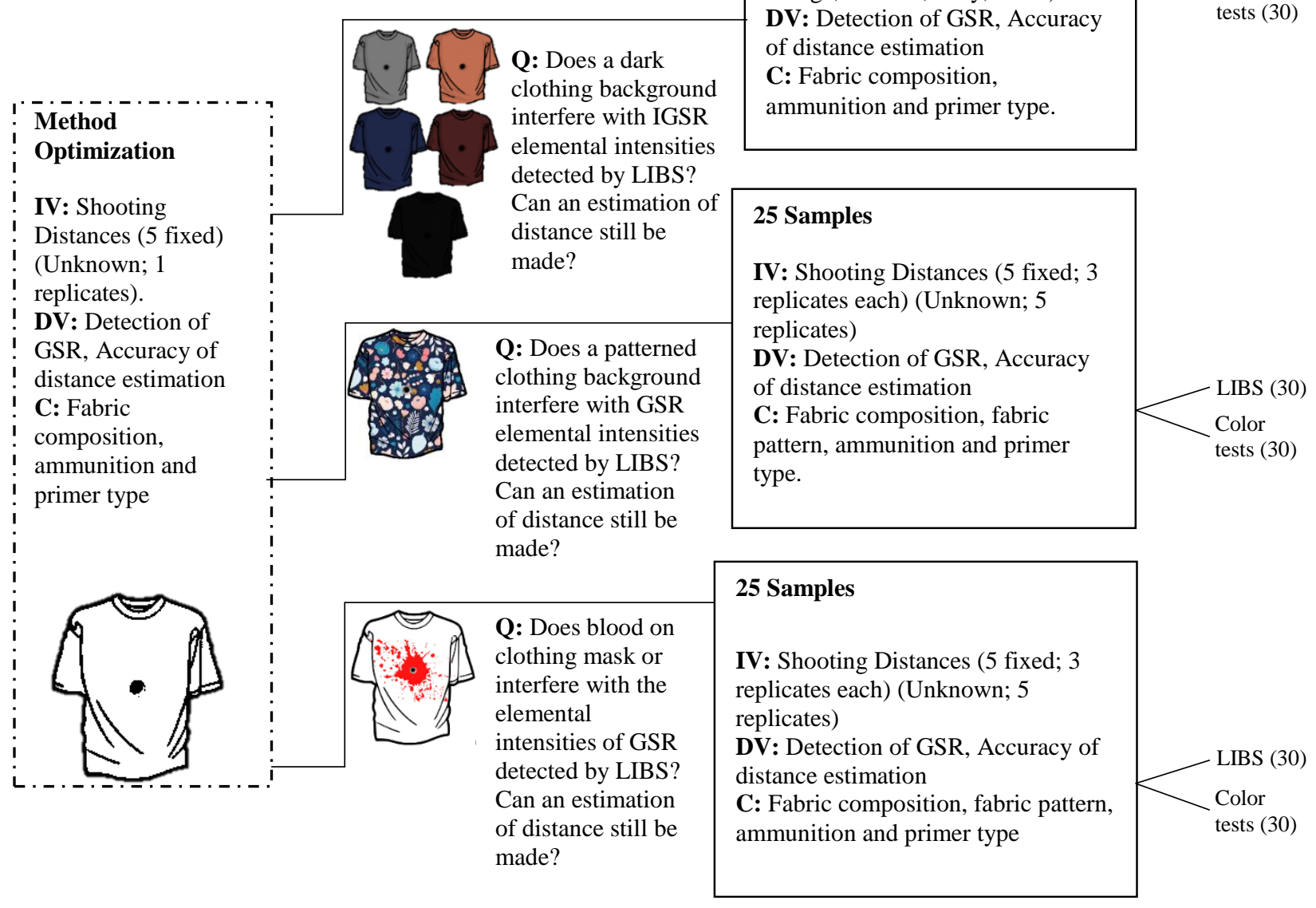

\section{Samples}

IV: Shooting Distances (5 fixed; 3 replicates each) (Unknown; 3 replicates). Clothing color (grey, orange, maroon, navy, black) DV: Detection of GSR, Accuracy of distance estimation C: Fabric composition, ammunition and primer type.

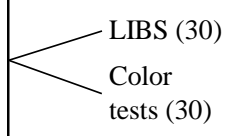

Q: Does a dar distance still be made?

Q: Does a patterned clothing background interfere with GSR elemental intensities detected by LIBS? Can an estimation of distance still be made?

Q: Does blood on clothing mask or interfere with the elemental intensities of GSR detected by LIBS? Can an estimation distance still be made?

Figure 7. Experimental design for shooting distance determination by analysis of IGSR using LIBS including sample types, variables, and analyses performed for each sample. $(\mathrm{IV}=$ independent variable, $\mathrm{DV}=\mathrm{dependent}$ variable, $\mathrm{C}=$ constant variable, $\mathrm{Q}=$ question)

\subsection{Spectrochemical Mapping Using Laser Induced Breakdown Spectroscopy as a More Objective Approach to Shooting Distance Determination}

Part of the following chapter, sections 3.2. and 3.4., is an adaptation of a previously published article (2019:

Pyl, C. Vander; Ovide, O.; Ho, M.; Yuksel, B.; Trejos, T. Spectrochemical Mapping Using Laser Induced Breakdown Spectroscopy as a More Objective Approach to Shooting Distance Determination. Spectrochim. Acta - Part B At. Spectrosc. 2019. https://doi.org/10.1016/j.sab.2018.12.010. 


\subsubsection{Methods and Materials}

\subsubsection{Firearms and Ammunitions}

A 9 mm Springfield XD9 firearm (manufactured in Croatia) and Rossi Revolver .357 Magnum firearm (manufactured in Brazil) were used for shootings and sample collections. The ammunition

for the Springfield XD9 consisted of Starline brass $9 \mathrm{~mm}$ luger cartridge cases reloaded with Remington 11/2 small pistol primers, Winchester 231 grain powder, and Speer $9 \mathrm{~mm}$ total metal jacket (TMJ) bullets. The ammunition used for the Rossi Revolver was 38 Special 158 grain with a lead round nose bullet.

\subsubsection{Sample Preparation and Collection}

Fabric in varying colors (patterned and plain grey, orange, maroon, navy, black, all 100\% cotton) was obtained from a retail store. The fabric was cut into $20 \mathrm{~cm}$ by $27 \mathrm{~cm}$ rectangles. All samples were placed in manila folders, with a white piece of copy paper on either side, and then folded in pre-labeled clean paper. The prepared samples were stored in a sealed plastic container at the laboratory until shooting and collection.

All shootings were performed at the West Virginia University Forensic and Investigative Sciences ballistics laboratory under controlled environmental conditions. Each fabric sample was hung on a self-healing shooting block using push pins and a clean manila folder support to prevent cross contamination. The distance from the muzzle of the firearm to the sample was set using a measuring tape and floor markers before each shooting. It was ensured that the individual handling and storing the samples was not the same individual performing the shootings.

A total of 45 samples (15 grey, 30 patterned) were shot at known distances and used as calibration sets. The distances used for calibration purposes were contact, 6 inches, 12 inches, 24 inches, and 36 inches; each distance was repeated three times for both grey and patterned fabrics. The grey calibration set was shot with pistol, while the pattern sets were shot with a pistol and a revolver. A total of 28 unknown samples were collected; 8 grey, 8 patterned, 3 orange, 3 maroon, 3 navy, and 3 black. The analyst was blind to the true distance of the unknown samples while performing testing.

Nitrile gloves (Fischer Scientific; NH) and lab coats (Fischer Scientific; NH) were worn during sample handling, preparation, collection, and analysis. Gloves were changed between handling 
each new sample. Blank controls were analyzed in triplicate for each fabric type to evaluate any potential interferences.

\subsubsection{Experimental Set $U p$}

The experimental setup was designed to allow for subsequent analysis on the same fabric sample, first by LIBS, then by traditional physical examination, and modified Griess and sodium rhodizonate chemical color tests. In development of the sequence of experiments, practicality of analysis and concern for sample destruction were also considered. The ablation stage was moved fast while the laser was fired across the surface of the fabric to interact with the GSR while minimizing damage of the textile and preventing disturbance of surrounding GSR patterns. Indeed, the damage left on the fabric was almost invisible to the naked eye and the removal of soot and GSR were negligible in comparison to the overall macro-pattern of the distribution of GSR around the entrance hole.

\subsection{LIBS Instrumentation, Parameters, and Method}

A J200 Tandem Model (Applied Spectra; Freemont, CA) LIBS operating with a $266 \mathrm{~nm} 10 \mathrm{~ns}$ Neodymium doped Yttrium Aluminum Garnet laser and 6 channel Czerny-Turner spectrometer was used for analysis. The clothing samples were secured to a custom-made ablation stage that sits on top of the cell in the ablation chamber, with a non-interfering double-sided adhesive tape (Figure 8). The extra length of the fabric was secured to the metal ablation stage using magnets. The stage was covered with benchkote paper (Fisher Scientific, NH) to prevent contamination from the metal surface. With the camera focused on the area closest to the bullet hole, the laser was scanned across the fabric using a continuous straight ablation line of $100 \mu \mathrm{m}$ by $5 \mathrm{~mm}$, and the signal was accumulated for 85 shots. The laser was fired with an energy of $9.4 \mathrm{~W} / \mathrm{cm}^{2}$, with a frequency of $5 \mathrm{~Hz}$, and a velocity of $0.3 \mathrm{~mm} / \mathrm{s}$. This ablation pattern was repeated 26 consecutive times resulting in a total ablation line of $130 \mathrm{~mm}$ away from the center of the bullet hole. A summary of the LIBS parameters can be seen below in Table 1. 


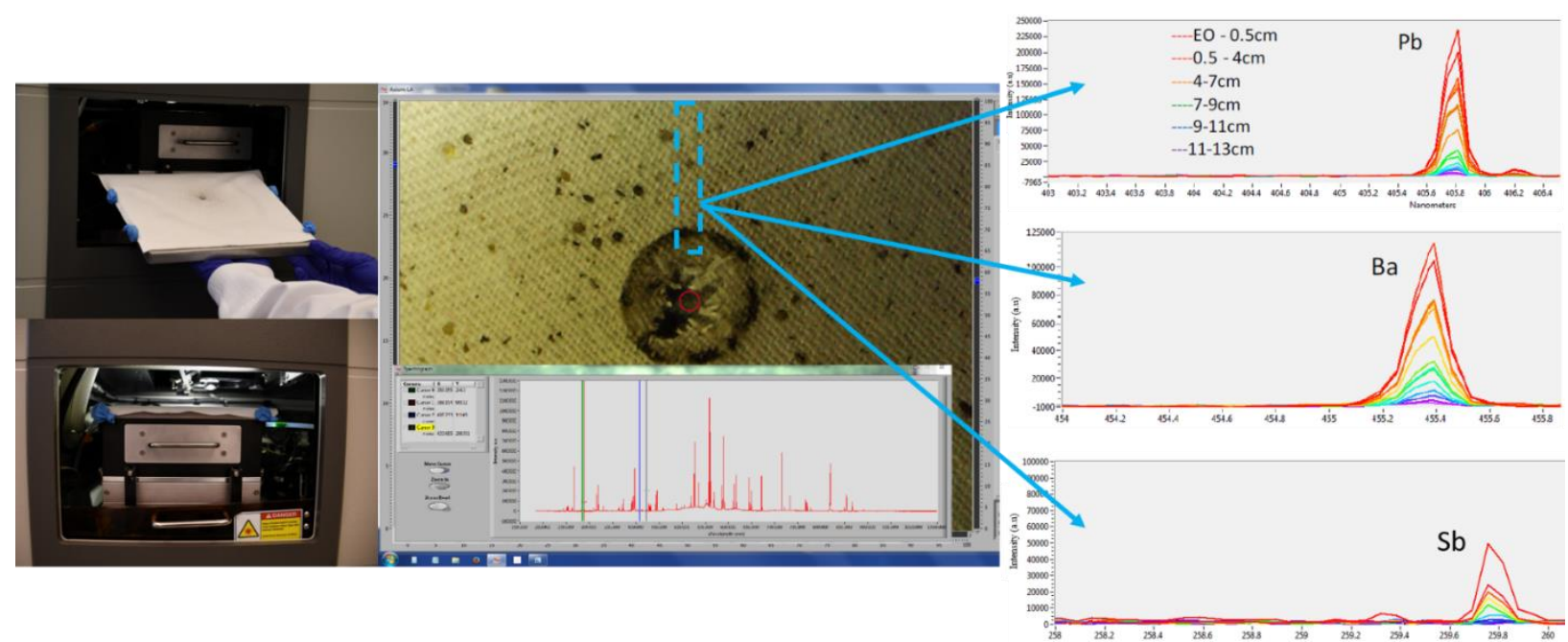

Figure 8. LIBS experimental procedure. Custom-made ablation plate setup utilized for sample analysis (left), LIBS area of ablation for analysis (middle), and examples of spectra obtained for elements of interest (right,from top to bottom: $\mathrm{Pb}$ (405.8 $\mathrm{nm}$, I), Ba (493.4 nm, II), Sb (259.8 nm, I)).

Table 1

LIBS Parameters of Line Acquisition for Shooting Distance Determination

\begin{tabular}{ll}
\hline Parameter & Measure \\
\hline Ablation Pattern & $(20 \mathrm{x}) 5 \mathrm{~mm}$ vertical line $\rightarrow$ Total of $100 \mathrm{~mm}$ \\
Spot Size & 100 \\
Number of shots per $5 \mathrm{~mm}$ line & 85 \\
Pulse repetition rate & $5 \mathrm{~Hz}$ \\
Pulse Energy & $40 \%$ \\
Stage Velocity & $0.3 \mathrm{~mm} / \mathrm{s}$ \\
Gate Delay & 0.5
\end{tabular}

A Box-Behnken response surface experimental design was used to find ablation parameters that maximize SNR while minimize samples to sample variance (precision). The optimal parameters and ablation pattern were tested on standards prepared by spiking Whatman paper \#42 (Fischer Scientific, $\mathrm{NH}$ ) and white cotton fabrics with 50 ug to $50 \mathrm{ng}$ of $\mathrm{Pb}, \mathrm{Ba}$, and $\mathrm{Sb}$. These known concentration solutions were used to determine limits of detection for elements of interest. The limits of detection were calculated using three times the standard deviation of the background and dividing it by the slope of the calibration curve, as recommended in literature ${ }^{70,71}$ then confirming by measuring standard samples at concentrations near the expected LOD. A preliminary validation was then conducted on a set of fifteen white cotton fabric samples, shot at known distances. 


\subsection{LIBS Data Analysis}

Aurora software (Applied Spectra; Freemont, CA) was used for data pre-processing, including background subtraction, peak identification, and peak area integration. The selected elemental peaks of interest were $\mathrm{Sb}(259.8 \mathrm{~nm}, \mathrm{I}), \mathrm{Pb}(405.8 \mathrm{~nm}, \mathrm{I})$, and $\mathrm{Ba}(493.4 \mathrm{~nm}$, II). Additional emission lines were used for confirmation ( $\mathrm{Pb}$ (368.3 nm, I), Ba (455.4 nm, II), Ba (553.5 nm, I), $\mathrm{Ba}(614.1 \mathrm{~nm}, \mathrm{II}), \mathrm{Ba}(705.9 \mathrm{~nm}, \mathrm{I})$, and $\mathrm{Sb}(252.8 \mathrm{~nm}, \mathrm{I}))$. Other emission lines, including $\mathrm{Al}$ (396.1 nm, I), $\mathrm{Al}(309.3 \mathrm{~nm}, \mathrm{I}), \mathrm{Cu}(327.4 \mathrm{~nm}, \mathrm{I})$ and $\mathrm{Cu}(324.7 \mathrm{~nm}, \mathrm{I})$, were also monitored. The integrated spectral data was initially analyzed using Excel 2016 (version 15.24, Microsoft Corporation). Chemical maps and multivariate statistical methods, including principal component analysis and discriminant analysis, were performed on JMP Pro (version 14.0.0; SAS Institute Inc., $\mathrm{NC})$.

\subsection{Color Tests and Physical Examinations}

The procedures for the modified Griess test and sodium rhodizonate test, described by Dillon, were followed to perform chemical color tests on all clothing samples ${ }^{21,24}$. The distances determined by the color tests were used to cross validate the results determined by LIBS analysis. Limits of detection of color tests were conducted by depositing $100 \mu \mathrm{L}$ of solutions of known concentrations (10,000 to $200 \mu \mathrm{g} / \mathrm{ml}$ ) on Whatman paper and let it dry. The deposited dried amounts ranged therefore from $100 \mu \mathrm{g}$ to $2 \mu \mathrm{g}$. The limit of detection was estimated as the lowest deposited amount that produced a color reaction detectable to the naked eye. The experiment was conducted in four replicates and repeated in three different days by three different analysts.

\subsection{Color Test Reagents}

The modified Griess test was performed using sulfanilic acid (Lot \# BCBQ1007V; Sigma Aldrich, MO), alpha-naphthol (Lot \# 10190898; Alfa Aesar, MA), sodium nitrite (Lot \# A0267857, Acros Organics-Thermo Fisher Scientific, MA), acetic acid (Lot \# 171289; Fischer Scientific, NH), methanol (Lot \# 170983; Fisher Chemical, PA), and desensitized HP Everyday Photographic Paper (Model \#Q8723A, Palo Alto, CA). The sodium rhodizonate test was performed using sodium rhodizonate (Lot \# BCBR0492V; Sigma Aldrich, MO), sodium bitartrate (Lot \# BCBR3492V; Sigma Aldrich, MO), tartaric acid (Lot\# Y04A021; Alfa Aesar, MA), hydrochloric acid (Lot \# 167045; Fisher Scientific, NH), Whatman paper \#42 (Fisher Scientific, NH), and Search Power 
Spray Units (Sirchie Acquisition Company LLC, NC). All experiments were conducted in a Safeaire ${ }^{\circledR}$ ventilated fume hood (Fischer Hamilton, Manitowoc, WI).

\subsection{Physical Measurements}

Photographic documentation and physical measurements ${ }^{12}$ were taken on the clothing samples before and after color testing. The length and width of the bullet hole and soot pattern were measured. The number of visible gun powder particles on the sample were counted as well as the distance of the particles from the bullet hole. After the modified Griess test, the area of the orange color present on the photographic paper and the area of the color deposited by the gunpowder particles were measured using ImageJ (version 1.8.0.112, National Institutes of Health, MD). After application of the sodium rhodizonate test, the area of the purple color was also measured using the same ImageJ program.

\subsubsection{Results and Discussions}

One of the main objectives of this study was to evaluate if LIBS can be used as an analytical approach to modernize current presumptive testing used for bullet hole identifications and shooting distance estimations. Consequently, all samples measured by LIBS were also analyzed by the conventional analytical protocol used at crime laboratories, including physical examination and color tests.

\subsubsection{Firing Distance Prediction Using LIBS Analysis and Multivariate Methods}

\subsection{LIBS Method Optimization and Assessment of Selectivity and Limits of Detection}

The Box Behnken response surface experimental design was used to determine the optimal ablation parameters. Four levels were assessed, each with three different ranks (low, medium, high); pulse frequency ( $3 \mathrm{~Hz}, 5 \mathrm{~Hz}, 10 \mathrm{~Hz}$ ), laser energy (40\%, 60\%, 80\%), gate delay $(0.2 \mu \mathrm{s}, 0.5$ $\mu \mathrm{s}, 1.0 \mu \mathrm{s})$, and stage velocity $(0.2 \mathrm{~mm} / \mathrm{s}, 0.3 \mathrm{~mm} / \mathrm{s}, 0.4 \mathrm{~mm} / \mathrm{s})$. The best signal to noise, and least destruction of sample, was accomplished by using a gate delay of $0.5 \mu \mathrm{s}$, firing the laser at $40 \%$ energy $(6 \mathrm{~mJ})$ with a repetition rate of $5 \mathrm{~Hz}$, a spot size of $100 \mu \mathrm{m}$, and accumulating the signal while scanning the laser $5 \mathrm{~mm}$ across the sample at a speed of $0.3 \mathrm{~mm} / \mathrm{s}$. The same pattern was repeated 26 times, moving straight from the bullet hole to ensure the entire GSR distribution 
pattern was captured during analysis. Using this method, time of analysis for one sample was, on average, 30 minutes.

Background spectra were taken in triplicate for each fabric type to assess any interfering elemental emission lines at wavelengths of interest. Except for a Silicon $(252.8 \mathrm{~nm}, \mathrm{I})$ interference with $\mathrm{Sb}$ $(252.8 \mathrm{~nm})$, no interferences were observed. Antimony's second emission line at $259.8 \mathrm{~nm}$ did not display selectivity issues (Figure 9).
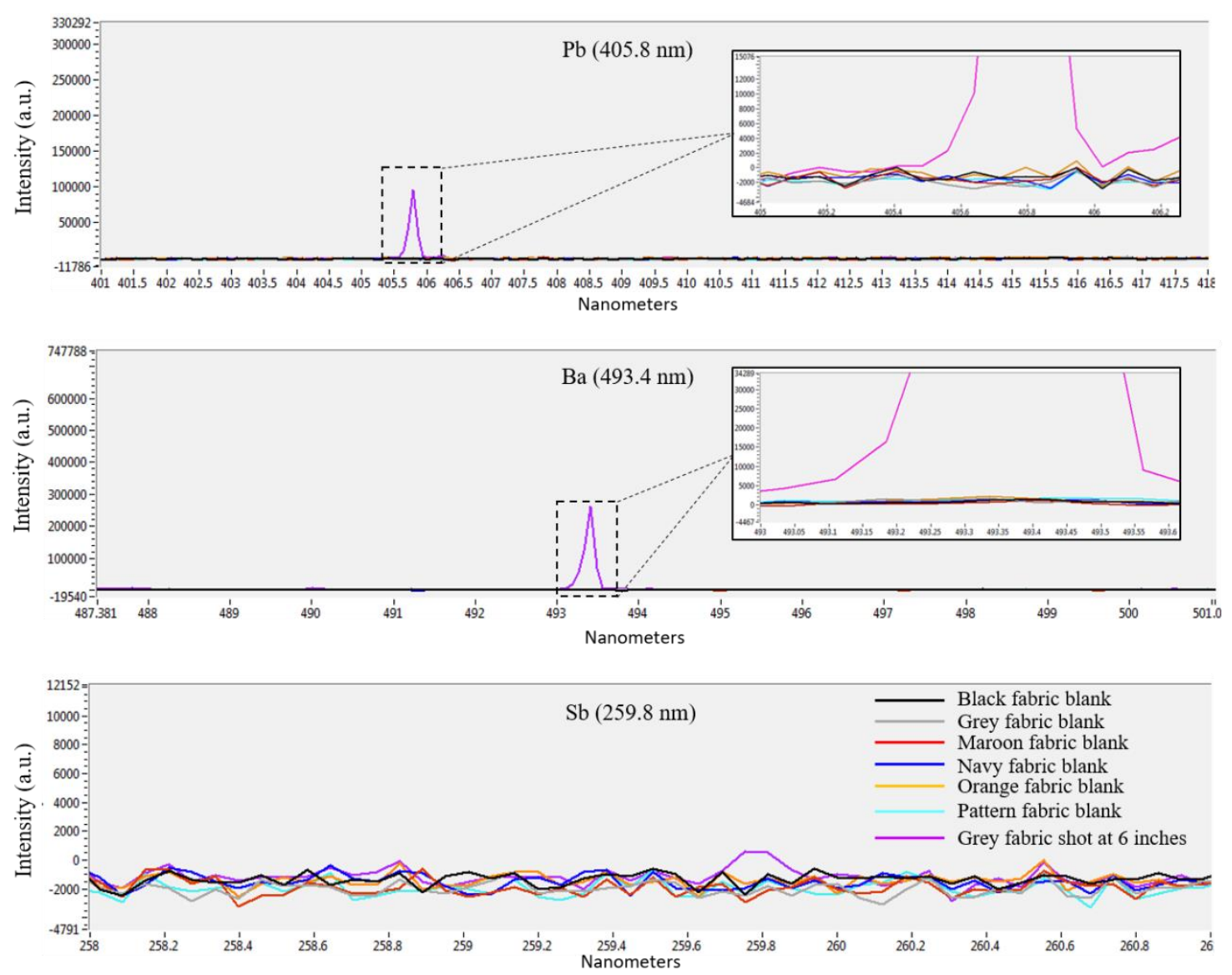

Figure 9. LIBS spectra of a clothing shot at 6 inches away and background spectra of the fabric prior shooting illustrating no interferences for elements of interest from negative control fabric samples.

Limits of detection (LOD) of LIBS and color tests were compared by depositing known amounts of elements of interest on Whatman paper and letting it dry prior ablation. Figure 10 illustrates the superior sensitivity of LIBS over color tests. LIBS absolute limits of detection were 2 to 4 orders of magnitude better than color tests, for lead and barium respectively. It's recognized that color test limits of detection could be improved by instrumental detection of the color rather than relying on visual detection. Nonetheless, the human eye detection was preferred as more representative of 
the method used by forensic examiners. In order to account for subjectivity in the color detection, the experiment was repeated by three different analyses and conducted in replicates. The reported values represent the average results and respective standard deviations. Moreover, LIBS can detect more elements associated with FDR, such as antimony, expanding current capabilities of chemical color tests in the field. (Table 2).
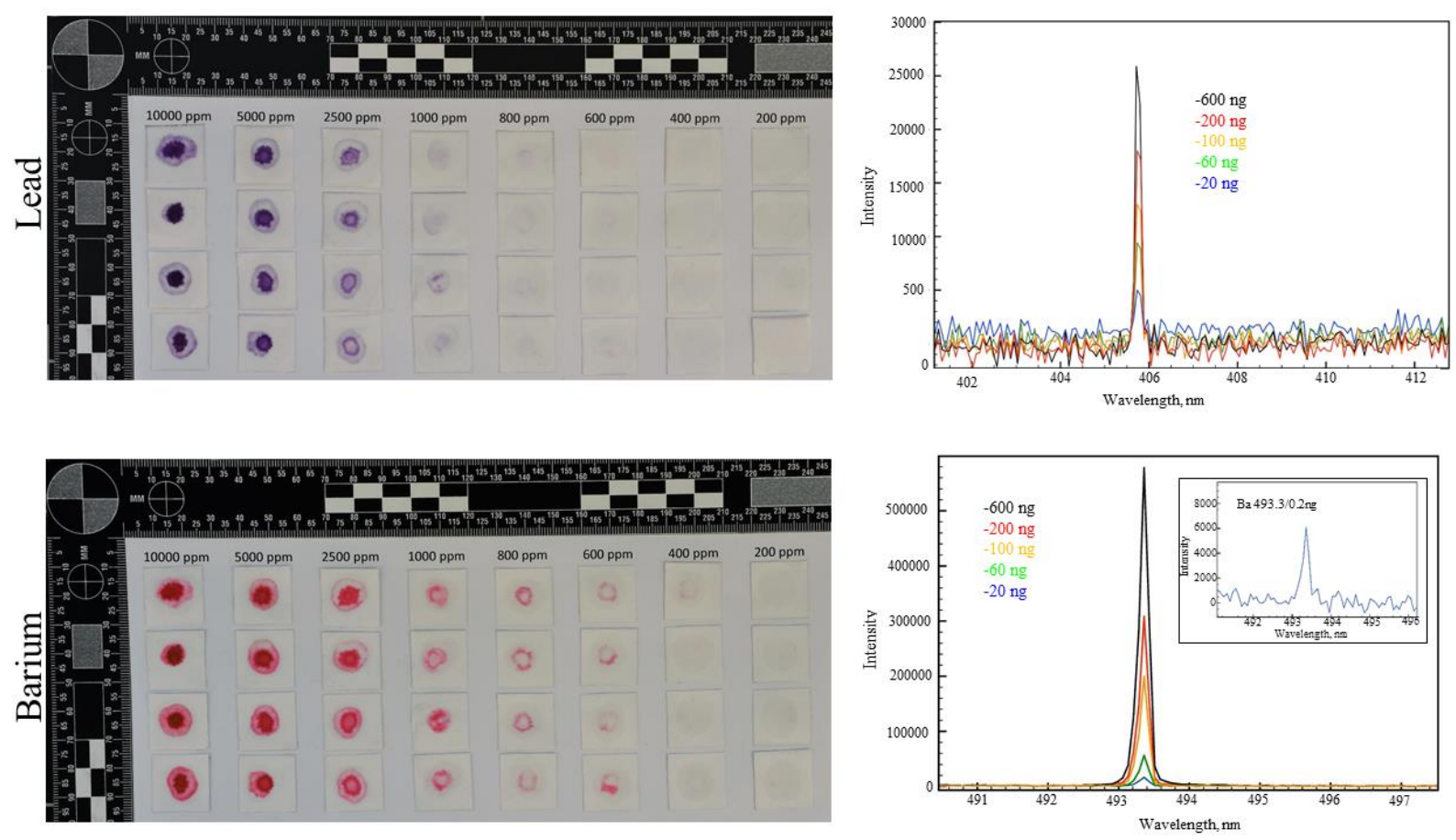

Figure 10. Results of limits of detection tests for the sodium rhodizonate assay (left), and LIBS (right), for lead (top) and barium (bottom), respectively. The inset shows a zoomed in look at Ba (493 nm) limit of detection of $0.2 \mathrm{ng}$. 
Table 2 Comparison of LOD's for Sodium Rhodizonate test and LIBS method

\begin{tabular}{llll}
\hline \multirow{2}{*}{ Species } & \multicolumn{3}{c}{ LIBS } \\
\hline Lead $(\mathrm{Pb})$ & $368.3(\mathrm{I})$ & $70 \pm 3$ & $7,000 \pm 1,000$ \\
& 405.7 (I) & $30 \pm 4$ & \\
Barium $(\mathrm{Ba})$ & 455.4 . (II) & $0.70 \pm 0.08$ & \\
& 493.4 (II) & $0.20 \pm 0.02$ & $5,000 \pm 1,000$ \\
& 553.5 (I) & $1.1 \pm 0.04$ & \\
& 614.1 (II) & $1.4 \pm 0.04$ & \\
& 705.9 (I) & $2.1 \pm 0.1$ & \\
Antimony $(\mathrm{Sb})$ & 252.8 (I) & $440 \pm 80$ & N/A \\
& 259.8 (I) & $220 \pm 13$ & \\
Copper $(\mathrm{Cu})$ & 324.7 (I) & $40 \pm 6$ & N/A \\
& 327.4 (I) & $20 \pm 4$ & \\
Aluminum $(\mathrm{Al})$ & 309.3 (I) & $50 \pm 6$ & N/A \\
& 396.2 (I) & $30 \pm 2$ &
\end{tabular}

\subsection{Chemical Maps for Shooting Distance Determinations}

Shooting distances for the calibration curves were chosen to represent gunshot residue patterns that are commonly associated with contact, close range (6 inches \& 12 inches), and long range (24 inches \& 36 inches) shootings. The integrated spectral data collected from shot fabrics was preprocessed to visualize the spatial changes on elemental composition relative to the firing distance. A bar graph of intensity versus ablation distance from bullet hole $(\mathrm{cm})$ was created for each element of interest $(\mathrm{Pb}, \mathrm{Ba}, \mathrm{Sb})$, representing the accumulated area intensity of each $5 \mathrm{~mm}$ increment as the laser moved away from the orifice. For each of the calibration sets, all distances (contact, 6 inches, 12 inches, 24 inches, and 36 inches) were displayed on the same graph to illustrate the difference in chemical intensity patterns. Figure 11 shows a representation of $\mathrm{Pb}$ $(405.8 \mathrm{~nm})$ for one set of grey calibration samples. The contact sample shows the highest initial intensity closest to the bullet hole, with a sharp decline in intensity that levels out around $4 \mathrm{~mm}$. The short-range distance samples (6 inches \& 12 inches) show lower initial intensities but have a more gradually decline before leveling out at $8.5 \mathrm{~mm}$. Long range distances ( 24 inches \& 36 inches) have consistent low intensity levels from the entrance hole to the end of the fabric, with 
slightly higher levels for 24 inches. It should be noted that some contact samples had lower initial intensities due to the large star shaped patterned surrounding the bullet hole (Figure 15). It is suspected that the large opening allowed for GSR to travel through the fabric, instead of being imbedded at the entry hole. To account for this issue when performing multivariate statistical methods, bullet hole dimensions were added to each sample as an additional qualifier.

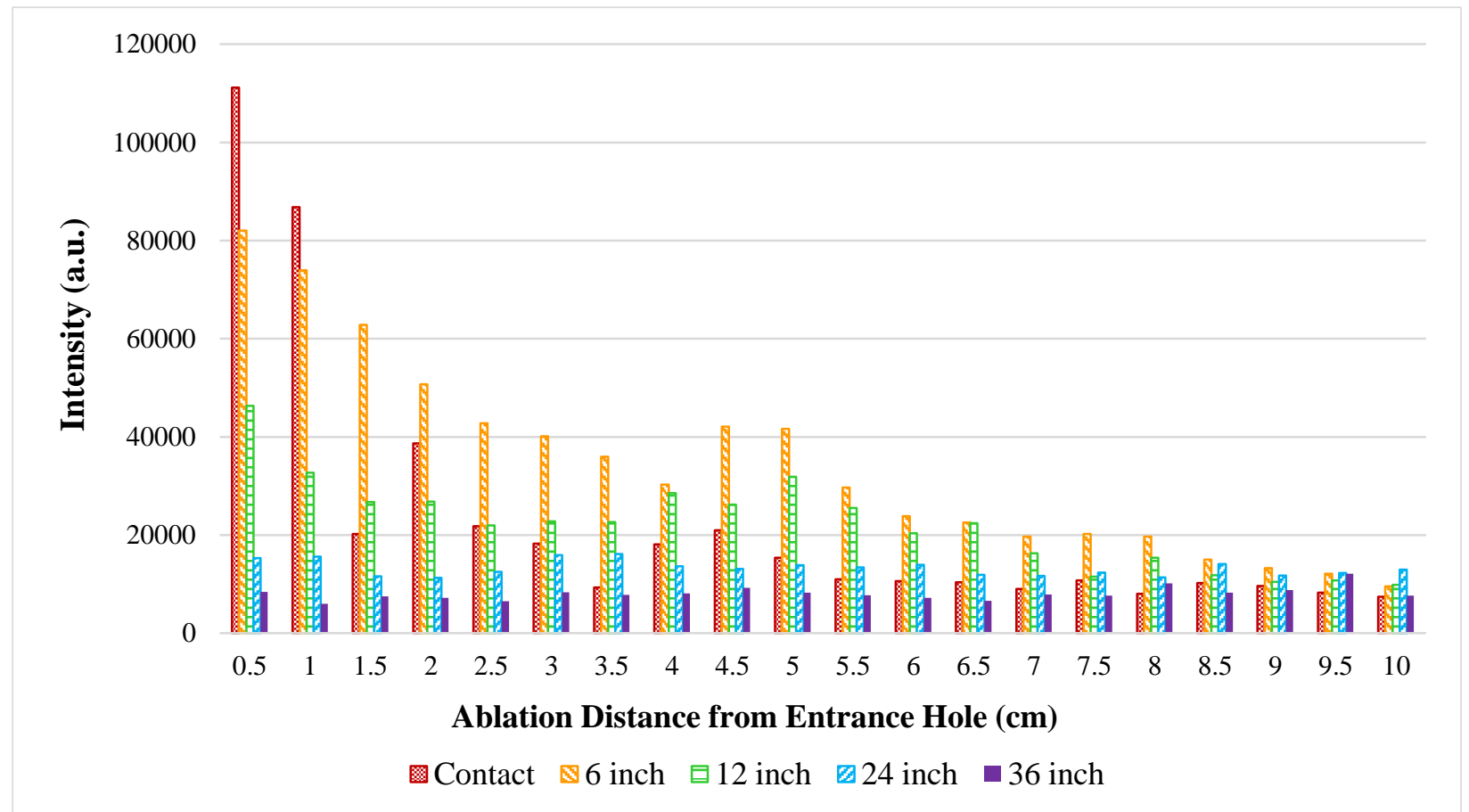

Figure 11. Bar graph representing intensity patterns for $\mathrm{Pb}(405.8 \mathrm{~nm})$ for a grey fabric calibration set. From left to right, the ablation from the entrance hole begins at $0.5 \mathrm{~mm}$ and increases by $0.5 \mathrm{~mm}$ until ending at $100 \mathrm{~mm}$. The legend represents the different shooting distance used for calibration (checkered red $=$ Contact, diagonal yellow $=6$ inches, horizontal green $=12$ inches, diagonal blue $=24$ inches, solid purple $=36$ inches).

As a supplementary and more advanced visualization of the data, chemical heat maps were made, using JMP Pro 14, for each element of interest. For instance, Figure 12 shows heat maps for the distribution of $\mathrm{Ba}(493.4 \mathrm{~nm}, \mathrm{II})$ at 4 different distances. The intensity data obtained for each sample was expanded $360^{\circ}$ to create concentric, uniform heat maps representative of the GSR pattern distributed around the bullet hole. The heat maps allow for a permanent visualization of IGSR distribution patterns. Similar to color tests, the darker colors represent a higher concentration of the analyte and the lighter colors represent lower concentrations of the analyte. Unlike color tests, the chemical maps contain spectral and numerical data, resulting in a more objective representation of the GSR pattern. Additionally, LIBS allows for the creation of heats maps for all elements of interest (lead, barium and antimony), which is unable to be achieved using color tests 
that are limited to nitrites and lead detection. These chemical maps are expected to facilitate explanation of the results to the trier of fact.
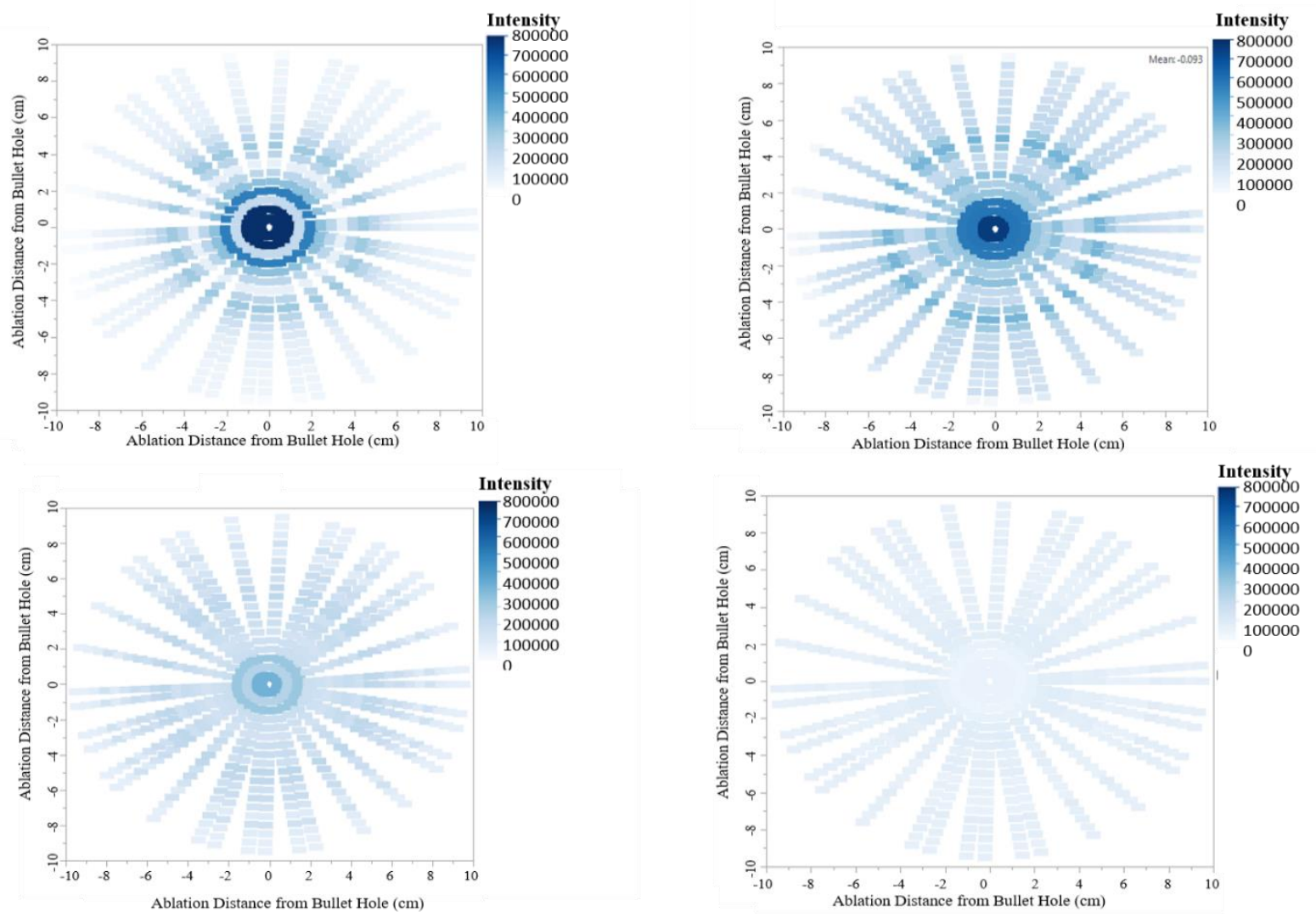

Figure 12. Heat maps of $\mathrm{Ba}(493.4 \mathrm{~nm})$ obtained from grey calibration set number 1. (a) contact; (b) 6 inches; (c) 12 inches; (d) 24 inches.

\subsection{Multivariate Methods}

Statistical approaches to classification of unknown distances included principal component analysis and multivariate discriminant analysis. As a first step, PCA plots were constructed using the spectral intensities found for the entirety of the $5 \mathrm{~mm}$ ablation increments; $130 \mathrm{~mm}$ total. Each increment had numeric outputs from the accumulated integrated areas of the multiple elements and emission lines. All three replicates of calibration curve samples were used in the construction of the PCA plots. Grey calibration curve sets were assessed with 3 grey, 3 orange, 3 maroon, 3 navy, and 3 black unknown samples. Patterned calibration curve samples were assessed with 5 patterned and 5 grey unknown samples. When using the full $130 \mathrm{~mm}, 71.4 \%, 76.5 \%$, and $83.3 \%$ of the variation of the grey and patterned (pistol and revolver) data sets were captured, respectively. In an attempt to increase the amount of variability captured, the data was reassessed and it was 
determined that any data after $100 \mathrm{~mm}$ of ablation provided little information. The elemental intensities, for all distances, were too similar past this ablation distance, and only added unnecessary noise to the data set. When the PCA plots were reconstructed with the reduced data set and included the bullet hole qualifier, the variability captured increased to $79.1 \%, 83.8 \%$, and 90\% for the grey and patterned (pistol and revolver) calibration sets, respectively.

The first two principal components, along with the bullet hole qualifier, were used as the variables to perform discriminant analysis and construct a canonical plot (Figure 13 and Figure 14). Discriminant analysis is a supervised hard classification method that uses a single vector, or canonical, to separate the data in order to have the largest variability between categories and the smallest variability within categories. In the case of numerous categories, a central point is utilized instead of a vector. The model attempts to maximize the distance between each individual data point and the central data point while keeping the scatter of each category minimized. To compensate for the small number of samples in this study, regularized discriminant analysis was applied. In both cases, the calibration curve samples were used as the training set and the unknown samples were used as the validation, or test set. Classifications of unknowns were determined by the first and second predictions made by the discriminant analysis model. Sixteen unknown samples (grey, orange, maroon, navy, black, pattern) had a first prediction of 12 inches and a second prediction of 6 inches. Therefore, the determined distance range for these 16 samples was 6 inches-12 inches (close range shot) with an emphasis closer to 12 inches. The model predicted 7 unknown samples (patterned) to be 24 inches with a second prediction of 36 inches and 5 samples (grey and patterned) to be 36 inches with a second prediction of 24 inches. No misclassifications of LIBS spectral patterns were found in the validation set. A misclassification occurred when the determined distance range did not include the true distance or the determined distance range was too large. A distance range was not needed to classify an unknown sample that was a contact shot due to the characteristic star shaped pattern. 


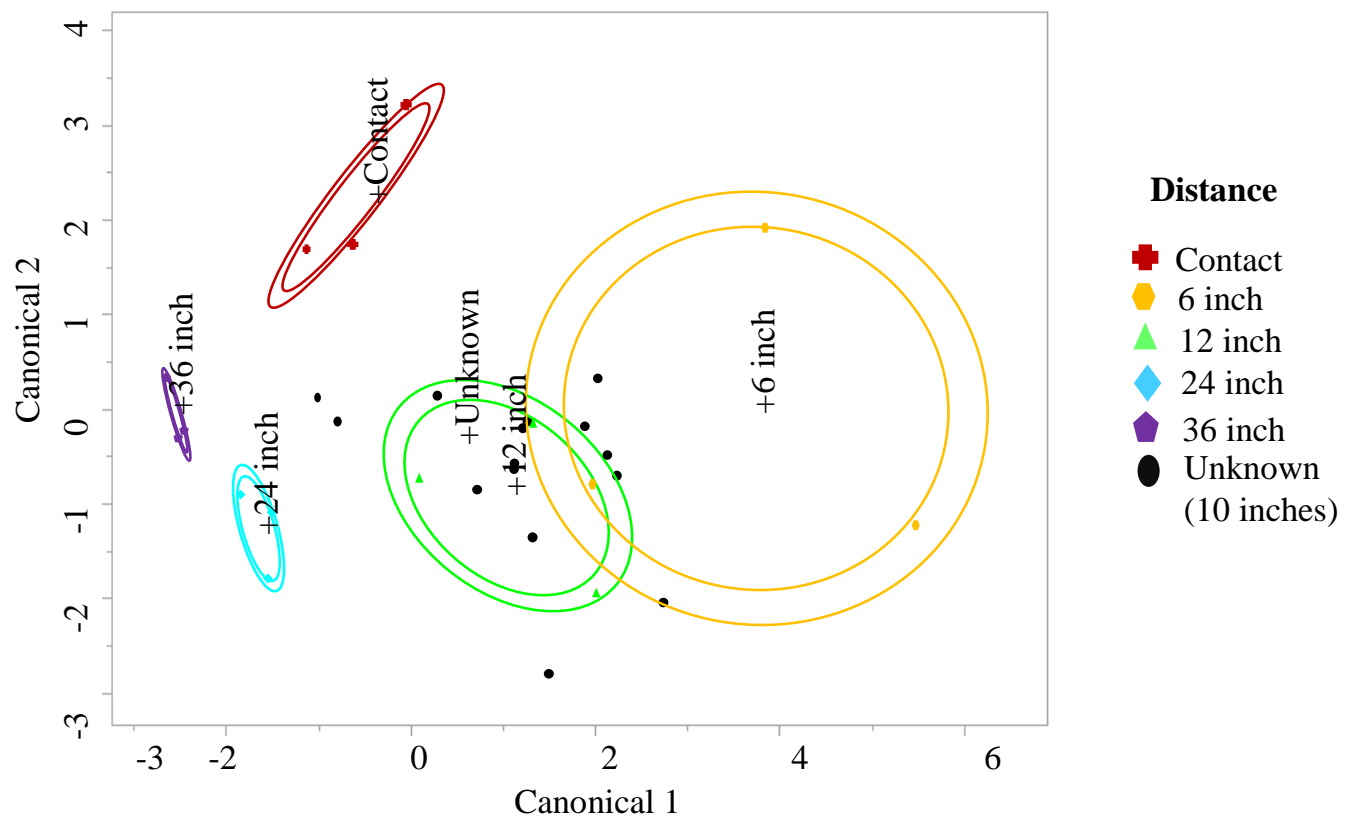

Figure 13. Canonical plot for grey calibration samples with grey unknowns (1,2, and 3), orange unknowns $(1,2$, and 3 ), maroon unknowns (1,2, and 3), navy unknowns (1,2, and 3), and black unknowns (1,2, and 3). All unknowns have a true shooting distance of 10 inches. (+ represents the multivariate mean for each shooting distance, outer ellipses represent $95 \%$ confidence interval for each shooting distance, and inner ellipses represent a 50\% contour of observations for each shooting distance.)

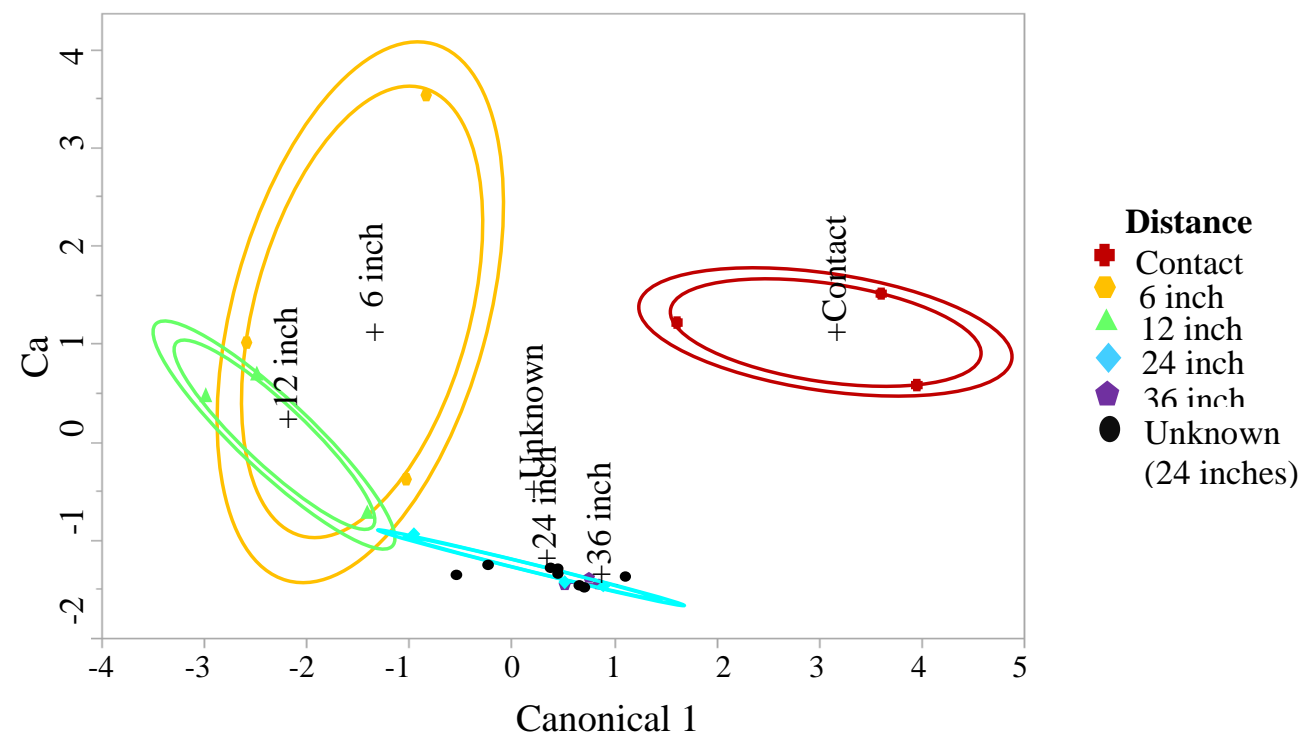

Figure 14. Canonical plot for patterned calibration samples with patterned unknowns (1,2,3,4, and 5), grey unknowns $(1,2,3,4$, and 5). All unknowns have a true shooting distance of 24 inches. (+ represents the multivariate mean for each shooting distance, outer ellipses represent $95 \%$ confidence interval for each shooting distance, and inner ellipses represent a $50 \%$ contour of observations for each shooting distance.) 


\subsubsection{Firing Distance Predictions using Color Tests}

\subsection{Interpretation of Physical and Visual Examination and Chemical Color Tests}

A typical physical examination of a fired clothing includes the comparison of bullet hole dimensions, soot dimensions, and gun powder distribution and density around the orifice. A calibration set is normally shot with the same suspect firearm and ammunition on similar fabrics as the questioned item. Then, the observations are visually compared, and the examiners decides to what known distance the questioned sample is most similar. The process unfortunately is very variable and dependent on the examiner experience. Table 3 illustrates the measurements taken for calibration sets and unknown samples (grey, orange, maroon, navy, and black). Darker fabrics including the maroon, navy, black, and patterned, made it difficult to accurately complete physical measurements. The wide variability of between and within physical properties for these samples resulted in a large number of misclassifications and inconclusive results (28.6\% and $35.7 \%$, respectively).

All 73 samples in this study were subjected to both the modified Griess test and the sodium rhodizonate test (Table 4). Positive controls were completed on the corners of the prepared Griess test sheets prior to analysis on a sample. It should be noted that LIBS ablations did not interfere with modified Griess distribution patterns. The laser moving quickly across the surface of the sample did not disrupt surrounding soot or gunpowder particles. A microscopic ablation line, with a width of $100 \mu \mathrm{m}$, is created only on the fabric's surface but does not penetrate the fabric. Additionally, this is confirmed in Figure 15. where no disruption in pattern can be seen in the modified Griess test results. The calibration curve samples showed consistent Griess test results for all replicate samples. Performing the Griess test on the orange and maroon colored fabrics helped to enhance the gunshot residue patterns on the fabric that were previously masked by the background color of the fabric. However, the navy and black fabrics reacted with the reagents of the Griess test and caused an orange color to appear on the entirety of the photographic paper (Figure 16). The interference was confirmed by analyzing blank negative control samples of the black and navy fabrics by the Griess test, where an orange reaction was observed even in the

absence of FDR. While the color produced by the navy fabric was fainter than that of the black fabric, both interferences compromised the measurements. The fabric interference and lack of reproducibility prevented a reliable distance estimation for black or navy samples. 
To keep the integrity of the sample intact, the sodium rhodizonate test was performed using the transfer method with Whatman paper \#42. The sodium rhodizonate test was not sensitive enough for the type of ammunition employed in the pistol experiments. This is expected to be a result of the low concentrations of lead in the primer which produced a pattern of lead residues below the color test LOD (Figure 10). Since the ammunition used a Total Metal Jacket (TMJ), the main source of lead is anticipated to originate from the primer. Ongoing primer characterization studies in our group have shown that the Remington primer used in the pistol ammunition had lower lead concentration than other standard leaded ammunitions. In general, color test measurements showed a large variability, and limitations of sensitivity and selectivity that prevented consistent estimation of shooting distances.

\subsubsection{True Shooting Distance: Assessment of Accuracy of the Methods}

When reconstructing a crime scene involving a firearm it can be crucial to determine whether a shooting was an accident, a suicide, or a homicide. When deciding between these options, shooting distance determination becomes important; was it a contact, close range, or long range shot? Rather than a specific distance, a range of distances is typically reported. Therefore, the previously classified samples were stated in ranges, rather than one specific distance. A proper classification was considered when the true shooting distance falls into a 1 increment distance range; contact, 6 inches-12 inches, 12 inches-24 inches, 24 inches-36 inches. An incorrect classification was documented when the reported range falls outside the true distance, or the range spans more than one increment. Out of the 28 unknown samples tested in this study, $100 \%$ of them were determined to be correctly classified when using the proposed LIBS method and statistical analyses. On the other hand, only $35.7 \%$ of the samples were correctly classified when relying solely on physical measurements and $78.6 \%$ when using traditional color tests. Table 5 shows a breakdown of the results for each sample by the technique used. The LIBS predictions have bolded numbers representing the model's first predictions and non-bolded numbers for the model's second predictions. The results of this study establish the forensic value of implementing the proposed LIBS method as a technique for shooting distance determinations and GSR pattern visualization in crime laboratories. 
Table 3

Physical measurements taken of all samples before application of color tests $(P=$ pistol, $R=$ revolver, $L=$ length, $W=$ width, $U=$ unknown (True distance: 12 inches for colors, 24 inches for patterned), Cal=calibration N/A= not visible for measurement)

\begin{tabular}{|c|c|c|c|c|c|}
\hline Sample & Distance & $\begin{array}{l}\text { Bullet hole LxW } \\
(\mathrm{cm})\end{array}$ & Soot LxW (cm) & $\begin{array}{l}\text { Gun Powder LxW } \\
\text { (cm) }\end{array}$ & $\begin{array}{l}\text { Gun } \\
\text { Particle } \\
\text { Count }\end{array}$ \\
\hline Cal Grey Set $(P)$ & Contact & $0.5 \pm 0.2 \times 0.4 \pm 0.2$ & $5.4 \pm 0.3 \times 4.8 \pm 0.5$ & $4.8 \pm 4.3 \times 2.9 \pm 4.7$ & $35 \pm 60$ \\
\hline Cal Grey Set $(P)$ & 6 inches & $0.4 \pm 0.1 \times 0.5 \pm 0.1$ & $10.6 \pm 5.8 \times 9.7 \pm 4.6$ & $7.0 \pm 3.2 \times 7.5 \pm 3.8$ & $45 \pm 24$ \\
\hline Cal Grey Set $(P)$ & 12 inches & $0.5 \pm 0.3 \times 0.5 \pm 0.3$ & $10.7 \pm 0.6 \times 9.8 \pm 1.3$ & $6.4 \pm 2.1 \times 4.2 \pm 1.2$ & $76 \pm 48$ \\
\hline Cal Grey Set $(P)$ & 24 inches & $0.3 \pm 0.1 \times 0.4 \pm 0.0$ & N/A & $9.2 \pm 1.2 \times 7.5 \pm 0.9$ & $70 \pm 51$ \\
\hline Cal Grey Set $(P)$ & 36 inches & $0.1 \pm 0.3 \times 0.4 \pm 0.1$ & N/A & $7.8 \pm 0.7 \times 5.9 \pm 2.6$ & $18 \pm 15$ \\
\hline Cal Pattern Set $(P)$ & Contact & $0.9 \pm 0.4 \times 1.0 \pm 0.6$ & N/A & N/A & N/A \\
\hline Cal Pattern Set $(P)$ & 6 inches & $0.4 \pm 0.1 \times 0.4 \pm 0.2$ & N/A & N/A & N/A \\
\hline Cal Pattern Set $(P)$ & 12 inches & $0.3 \pm 0.2 \times 0.3 \pm 0.0$ & N/A & N/A & N/A \\
\hline Cal Pattern Set $(P)$ & 24 inches & $0.3 \pm 0.0 \times 0.3 \pm 0.0$ & N/A & N/A & N/A \\
\hline Cal Pattern Set $(P)$ & 36 inches & $0.2 \pm 0.1 \times 0.2 \pm 0.6$ & N/A & N/A & N/A \\
\hline Cal Pattern Set $(R)$ & Contact & $0.9 \pm 0.1 \times 0.8 \pm 0.1$ & $5 \pm 1.0 \times 5.2 \pm 1.0$ & N/A & N/A \\
\hline Cal Pattern Set $(R)$ & 6 inches & $0.5 \pm 0.1 \times 0.5 \pm 0.0$ & N/A & N/A & N/A \\
\hline Cal Pattern Set $(R)$ & 12 inches & $0.4 \pm 0.1 \times 0.4 \pm 0.1$ & N/A & N/A & N/A \\
\hline Cal Pattern Set $(R)$ & 24 inches & $0.2 \pm 0.1 \times 0.5 \pm 0.1$ & N/A & N/A & N/A \\
\hline Cal Pattern Set $(R)$ & 36 inches & $0.3 \pm 0.0 \times 0.4 \pm 0.1$ & N/A & N/A & N/A \\
\hline $\operatorname{Grey}(P)$ & $\mathrm{U} 1$ & $0.4 \times 0.5$ & $11.9 \times 10.9$ & $5.7 \times 7.5$ & 55 \\
\hline $\operatorname{Grey}(P)$ & $\mathrm{U} 2$ & $0.3 \times 0.4$ & $9.9 \times 8.2$ & $8.4 \times 3.5$ & 11 \\
\hline $\operatorname{Grey}(P)$ & U 3 & $0.6 \times 0.5$ & $10.0 \times 8.2$ & $11.3 \times 6.8$ & 29 \\
\hline Orange $(P)$ & $\mathrm{U} 4$ & $0.3 \times 0.4$ & $9.2 \times 10.0$ & $3.3 \times 4.8$ & 98 \\
\hline Orange $(P)$ & U 5 & $0.4 \times 0.3$ & $7.8 \times 8.8$ & $7.6 \times 3.1$ & 28 \\
\hline Orange $(P)$ & U 6 & $0.4 \times 0.6$ & $8.2 \times 9.1$ & $7.6 \times 6.7$ & 115 \\
\hline Maroon $(P)$ & U 7 & $0.4 \times 0.6$ & $8.5 \times 9.3$ & $4.6 \times 4.0$ & 48 \\
\hline Maroon $(P)$ & U 8 & $0.3 \times 0.4$ & $5.2 \times 4.4$ & $8.0 \times 4.3$ & 47 \\
\hline Maroon $(P)$ & U 9 & $0.3 \times 0.5$ & $6.6 \times 4.6$ & $4.6 \times 5.5$ & 52 \\
\hline $\operatorname{Navy}(P)$ & U 10 & $0.3 \times 0.4$ & N/A & $9.3 \times 8.8$ & 142 \\
\hline $\operatorname{Navy}(P)$ & U 11 & $0.5 \times 0.3$ & N/A & $9.8 \times 5.9$ & 9 \\
\hline $\operatorname{Navy}(P)$ & U12 & $0.5 \times 0.5$ & N/A & $6.8 \times 6.1$ & 126 \\
\hline Black $(P)$ & U 13 & $0.3 \times 0.5$ & N/A & $8.4 \times 9.4$ & 170 \\
\hline Black $(P)$ & U 14 & $0.3 \times 0.4$ & N/A & $7.2 \times 7.6$ & 3 \\
\hline Black $(P)$ & U 15 & $0.3 \times 0.4$ & N/A & $5.8 \times 7.9$ & 142 \\
\hline $\operatorname{Grey}(P)$ & U 16 & $0.4 \times 0.5$ & N/A & $8.9 \times 7.4$ & 18 \\
\hline $\operatorname{Grey}(P)$ & U 17 & $0.4 \times 0.4$ & N/A & $6.1 \times 1.2$ & 5 \\
\hline $\operatorname{Grey}(P)$ & U 18 & $0.4 \times 0.5$ & N/A & $10.9 \times 9.9$ & 13 \\
\hline $\operatorname{Grey}(P)$ & U 19 & $0.5 \times 0.5$ & N/A & $10.5 \times 5.8$ & 8 \\
\hline $\operatorname{Grey}(P)$ & U 20 & $0.6 \times 0.6$ & N/A & $12.3 \times 8.5$ & 17 \\
\hline Patterned (P) & U 21 & $0.4 \times 0.4$ & N/A & N/A & N/A \\
\hline Patterned $(P)$ & U 22 & $0.3 \times 0.2$ & N/A & N/A & N/A \\
\hline Patterned (P) & U 23 & $0.3 \times 0.3$ & N/A & N/A & N/A \\
\hline Patterned $(P)$ & U24 & $0.3 \times 0.3$ & N/A & N/A & N/A \\
\hline Patterned $(P)$ & U 25 & $0.2 \times 0.2$ & N/A & N/A & N/A \\
\hline Patterned (R) & U 26 & $0.4 \times 0.4$ & N/A & N/A & N/A \\
\hline Patterned (R) & U 27 & $0.4 \times 0.4$ & N/A & N/A & N/A \\
\hline Patterned (R) & U 28 & $0.5 \times 0.6$ & N/A & N/A & N/A \\
\hline
\end{tabular}



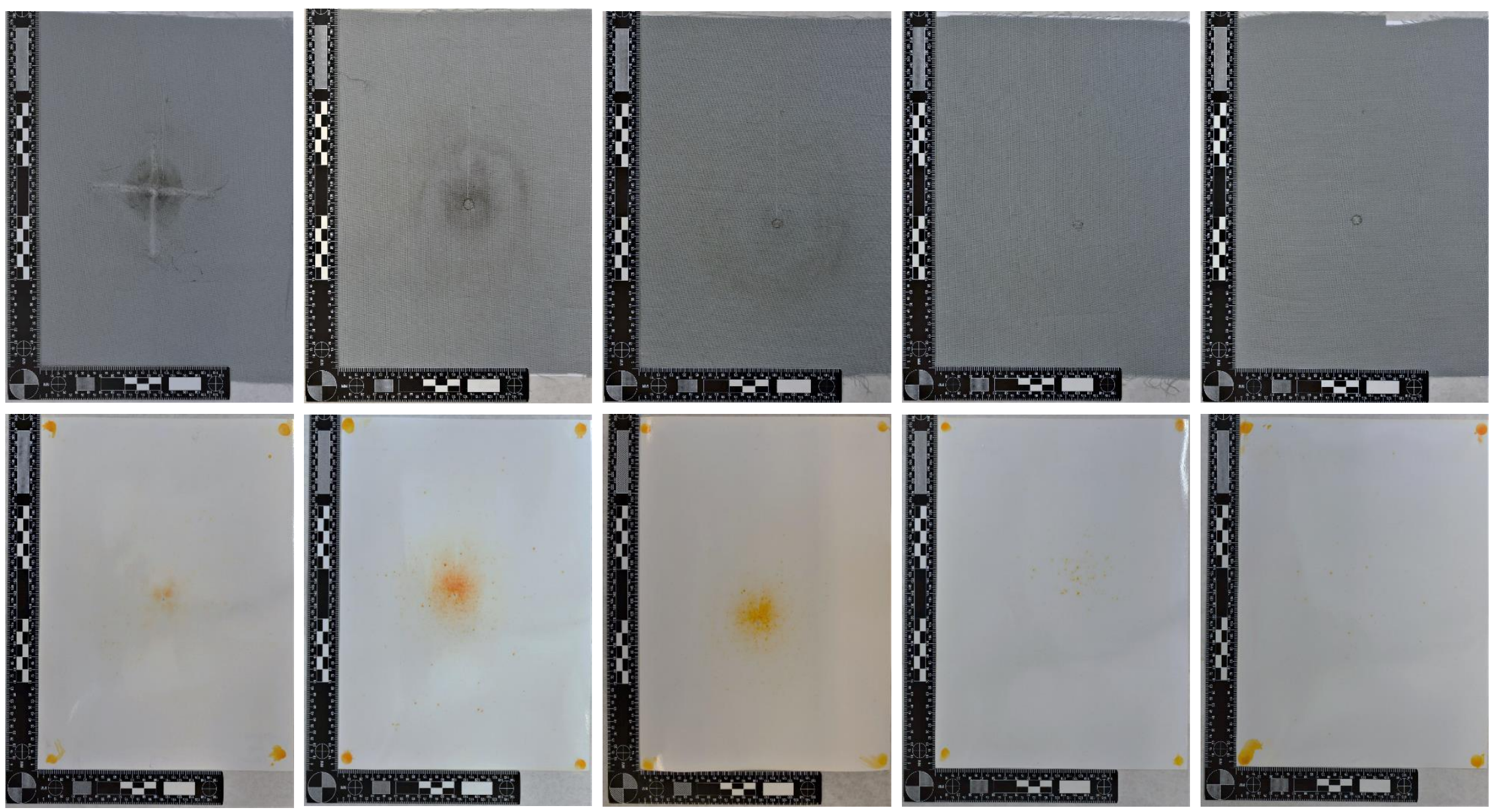

Figure 15. Photographs of original samples and results after the modified Griess test was performed on photographic paper (scale: $280 \mathrm{~mm} \times 150 \mathrm{~mm}$ ). Grey Calibration set (from left to right: contact, 6 inches, 12 inches, 24 inches, 36 inches). 


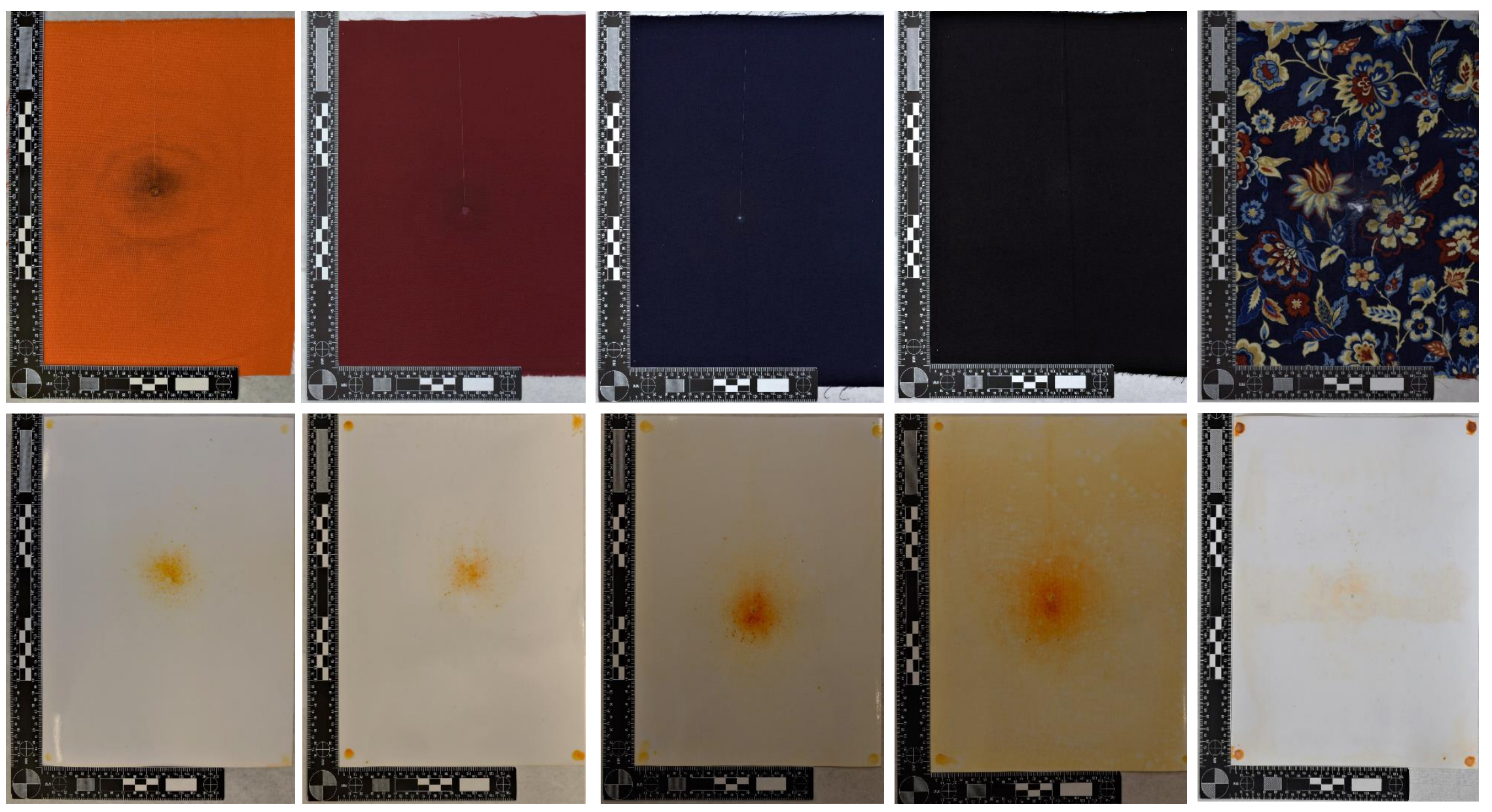

Figure 16. Photographs of original samples and results after the modified Griess test was performed on photographic paper (scale: $280 \mathrm{~mm} \times 150 \mathrm{~mm}$ ). Examples of unknowns (from left to right: orange, maroon, navy, black, and patterned). 
Table 4

Physical measurements taken for all samples after modified Griess and sodium rhodizonate tests $(P=$ pistol, $R=$ revolver, L=length, $W=$ width, Cal=calibration, $U=$ unknown(True distance: 12 inches for colors, 24 inches for patterned), $N / A=$ not visible for measurement)

\begin{tabular}{|c|c|c|c|c|c|}
\hline Sample & Distance & $\begin{array}{l}\text { Area Griess } \\
\text { Color } \\
\left(\mathbf{c m}^{2}\right) \\
\end{array}$ & $\begin{array}{l}\text { Area Griess } \\
\text { Particle } \\
\left(\mathbf{c m}^{2}\right) \\
\end{array}$ & $\begin{array}{l}\text { Area Sodium } \\
\text { Rhodizonate } \\
\text { Outer }\left(\mathbf{c m}^{2}\right) \\
\end{array}$ & $\begin{array}{l}\text { Area Sodium } \\
\text { Rhodizonate } \\
\text { Inner }\left(\mathbf{c m}^{2}\right) \\
\end{array}$ \\
\hline Cal Grey Set $(P)$ & Contact & $3.2 \pm 3.5$ & $50.6 \pm 67.8$ & N/A & $4.2 \pm 7.3$ \\
\hline Cal Grey Set $(P)$ & 6 inches & $20.1 \pm 4.3$ & $56.7 \pm 47.4$ & N/A & $1.4 \pm 1.7$ \\
\hline Cal Grey Set $(P)$ & 12 inches & $17.8 \pm 11.8$ & $70.3 \pm 79.7$ & N/A & $5.2 \pm 4.7$ \\
\hline Cal Grey Set $(P)$ & 24 inches & N/A & $53.76 \pm 76$ & N/A & $2.3 \pm 3.8$ \\
\hline Cal Grey Set $(P)$ & 36 inches & N/A & $49.4 \pm 69.9$ & N/A & N/A \\
\hline Cal Pattern Set $(P)$ & Contact & $11.9 \pm 3.1$ & $85.9 \pm 29.2$ & N/A & $11.1 \pm 9.2$ \\
\hline Cal Pattern Set $(P)$ & 6 inches & $29.1 \pm 3.8$ & $80.3 \pm 48.8$ & N/A & $7.6 \pm 9.9$ \\
\hline Cal Pattern Set $(P)$ & 12 inches & $7.1 \pm 4.6$ & $98.4 \pm 14.7$ & N/A & 9.9 \\
\hline Cal Pattern Set $(P)$ & 24 inches & N/A & $103.6 \pm 45.5$ & N/A & N/A \\
\hline Cal Pattern Set $(P)$ & 36 inches & N/A & $81.1 \pm 17.9$ & N/A & N/A \\
\hline Cal Pattern Set $(R)$ & Contact & $38.3 \pm 8.3$ & $70.2 \pm 20.0$ & $41.3 \pm 4.8$ & $6.4 \pm 3.0$ \\
\hline Cal Pattern Set $(R)$ & 6 inches & $44.6 \pm 9.7$ & $88.1 \pm 14.1$ & $46.9 \pm 30.9$ & $0.9 \pm 0.7$ \\
\hline Cal Pattern Set (R) & 12 inches & $24.8 \pm 20.5$ & $86.2 \pm 28.5$ & $22.0 \pm 19.1$ & $1.2 \pm 0.3$ \\
\hline Cal Pattern Set $(R)$ & 24 inches & $0.4 \pm 0.4$ & $111.9 \pm 37.7$ & $\mathrm{~N} / \mathrm{A}$ & $1.2 \pm 0.3$ \\
\hline Cal Pattern Set $(R)$ & 36 inches & N/A & $122.2 \pm 41.6$ & N/A & $1.0 \pm 0.1$ \\
\hline Grey $(P)$ & $\mathrm{U} 1$ & 21.2 & 96.45 & N/A & 1.89 \\
\hline $\operatorname{Grey}(P)$ & $\mathrm{U} 2$ & 17.6 & 106.6 & N/A & 0.32 \\
\hline Grey $(P)$ & $\mathrm{U} 3$ & 19.8 & 102.1 & N/A & 6.72 \\
\hline Orange $(P)$ & $\mathrm{U} 4$ & 27.5 & 52.5 & N/A & 1.26 \\
\hline Orange $(P)$ & $\mathrm{U} 5$ & 42 & 92.4 & N/A & 15.1 \\
\hline Orange $(P)$ & $\mathrm{U} 6$ & 24.5 & 60.9 & N/A & 8.1 \\
\hline Maroon (P) & U 7 & 13.9 & 97.2 & N/A & 0.6 \\
\hline Maroon (P) & $\mathrm{U} 8$ & 16.8 & 109.3 & N/A & 8.4 \\
\hline Maroon (P) & U 9 & 13.7 & 50.8 & N/A & 5.3 \\
\hline $\operatorname{Navy}(P)$ & U 10 & 21.9 & 38.7 & N/A & 9.3 \\
\hline $\operatorname{Navy}(P)$ & U11 & 23.8 & 35.2 & N/A & 10.9 \\
\hline $\operatorname{Navy}(P)$ & $\mathrm{U} 12$ & 27.3 & 63.1 & N/A & 7.8 \\
\hline Black $(P)$ & U 13 & 39.5 & 9.3 & N/A & 2.2 \\
\hline Black $(P)$ & U 14 & 29.8 & 97.6 & N/A & 20.4 \\
\hline Black $(P)$ & U 15 & 12.9 & 11.2 & N/A & 10.5 \\
\hline Grey $(P)$ & $\mathrm{U} 16$ & N/A & 112.5 & N/A & N/A \\
\hline Grey $(P)$ & U 17 & N/A & 65.0 & N/A & N/A \\
\hline $\operatorname{Grey}(P)$ & U 18 & N/A & 65.7 & N/A & N/A \\
\hline Grey $(P)$ & U 19 & N/A & 107.3 & N/A & N/A \\
\hline Grey $(P)$ & U 20 & N/A & 100.8 & N/A & N/A \\
\hline Patterned $(P)$ & $\mathrm{U} 21$ & 28.6 & 106.4 & N/A & N/A \\
\hline Patterned (P) & $\mathrm{U} 22$ & N/A & 96.8 & N/A & N/A \\
\hline Patterned $(P)$ & $\mathrm{U} 23$ & N/A & 120.4 & N/A & N/A \\
\hline Patterned (P) & U 24 & N/A & 222.6 & N/A & N/A \\
\hline Patterned $(P)$ & $\mathrm{U} 25$ & N/A & 146.0 & N/A & N/A \\
\hline Patterned (R) & U 26 & 0.9 & 127.0 & N/A & 1.0 \\
\hline Patterned (R) & $\mathrm{U} 27$ & N/a & 160.6 & N/A & 0.9 \\
\hline Patterned (R) & $\mathrm{U} 28$ & 0.3 & 128.3 & N/A & 1.0 \\
\hline
\end{tabular}


Table 5

Summary table of distance range classifications for the LIBS method, physical measurements, modified Griess and sodium rhodizonate color tests (RTL=range too large Bold \#= model's first prediction, Non-bold \#= model's second prediction)

\begin{tabular}{|c|c|c|c|c|c|c|c|}
\hline $\begin{array}{l}\text { Unknown Sample } \\
\text { Name }\end{array}$ & $\begin{array}{l}\text { LIBS/Discriminant } \\
\text { Analysis Distance } \\
\text { Range Classification }\end{array}$ & $\begin{array}{l}\text { Actual } \\
\text { Distance }\end{array}$ & $\begin{array}{l}\text { Correct Distance } \\
\text { Range by LIBS/ } \\
\text { Discriminant } \\
\text { analysis? }\end{array}$ & $\begin{array}{l}\text { Visual/Physical } \\
\text { Distance Range } \\
\text { Classification }\end{array}$ & $\begin{array}{l}\text { Correct Distance } \\
\text { Range by } \\
\text { Visual/Physical? }\end{array}$ & $\begin{array}{l}\text { Color Tests } \\
\text { Distance Range } \\
\text { Classification }\end{array}$ & $\begin{array}{l}\text { Correct } \\
\text { Distance Range } \\
\text { by Color Tests }\end{array}$ \\
\hline 1 Grey $(P)$ & 6-12 inches & 10 inches & Yes & 6-24 inches & RTL & 6-12 inches & Yes \\
\hline 2 Grey $(P)$ & 6-12 inches & 10 inches & Yes & 12-24 inches & No & 6-36 inches & RTL \\
\hline 3 Grey $(P)$ & 6-12 inches & 10 inches & Yes & 6-24 inches & RTL & 6-12 inches & Yes \\
\hline 4 Orange $(P)$ & 6-12 inches & 10 inches & Yes & 6-24 inches & RTL & 6-12 inches & Yes \\
\hline 5 Orange $(P)$ & 6-12 inches & 10 inches & Yes & 12-24 inches & No & 6-36 inches & RTL \\
\hline 6 Orange $(P)$ & 6-12 inches & 10 inches & Yes & $6-24$ inches & RTL & 6-12 inches & Yes \\
\hline 7 Maroon $(P)$ & 6-12 inches & 10 inches & Yes & 6-24 inches & RTL & 6-12 inches & Yes \\
\hline 8 Maroon $(P)$ & 6-12 inches & 10 inches & Yes & 12-24 inches & No & 6-36 inches & RTL \\
\hline $9 \operatorname{Maroon}(P)$ & 6-12 inches & 10 inches & Yes & 6-24 inches & RTL & 6-12 inches & Yes \\
\hline $10 \operatorname{Navy}(P)$ & 6-12 inches & 10 inches & Yes & 6-24 inches & RTL & 6-12 inches & Yes \\
\hline $11 \operatorname{Navy}(P)$ & 6-12 inches & 10 inches & Yes & 12-24 inches & No & 6-36 inches & RTL \\
\hline $12 \operatorname{Navy}(P)$ & 6-12 inches & 10 inches & Yes & 6-24 inches & RTL & 6-12 inches & Yes \\
\hline 13 Black $(P)$ & 6-12 inches & 10 inches & Yes & 6-24 inches & RTL & 6-12 inches & Yes \\
\hline 14 Black $(P)$ & 6-12 inches & 10 inches & Yes & 12-24 inches & No & 6-36 inches & RTL \\
\hline 15 Black $(P)$ & 6-12 inches & 10 inches & Yes & $6-24$ inches & RTL & 6-12 inches & Yes \\
\hline $16 \operatorname{Grey}(P)$ & 24-36 inches & 24 inches & Yes & 24-36 inches & Yes & 24-36 inches & Yes \\
\hline 17 Grey $(P)$ & 24-36 inches & 24 inches & Yes & 24-36 inches & Yes & 24-36 inches & Yes \\
\hline $18 \operatorname{Grey}(P)$ & 24-36 inches & 24 inches & Yes & 24-36 inches & Yes & 24-36 inches & Yes \\
\hline $19 \operatorname{Grey}(P)$ & 24-36 inches & 24 inches & Yes & 24-36 inches & Yes & 24-36 inches & Yes \\
\hline $20 \operatorname{Grey}(P)$ & 24-36 inches & 24 inches & Yes & 24-36 inches & Yes & 24-36 inches & Yes \\
\hline 21 Patterned $(P)$ & 6-12 inches & 12 inches & Yes & 24-36 inches & No & 24-36 inches & No \\
\hline 22 Patterned $(P)$ & 24-36 inches & 24 inches & Yes & 24-36 inches & Yes & 24-36 inches & Yes \\
\hline 23 Patterned $(P)$ & 24-36 inches & 24 inches & Yes & 24-36 inches & Yes & 24-36 inches & Yes \\
\hline 24 Patterned $(P)$ & 24-36 inches & 24 inches & Yes & 24-36 inches & Yes & 24-36 inches & Yes \\
\hline 25 Patterned $(P)$ & 24-36 inches & 24 inches & Yes & 24-36 inches & Yes & 24-36 inches & Yes \\
\hline 26 Patterned $(R)$ & 24-36 inches & 24 inches & Yes & 12-24 inches & No & 12-24 inches & Yes \\
\hline 27 Patterned $(R)$ & 24-36 inches & 24 inches & Yes & 24-36 inches & Yes & 24-36 inches & Yes \\
\hline 28 Patterned $(R)$ & 24-36 inches & 24 inches & Yes & 12-24 inches & No & 12-24 inches & Yes \\
\hline \multicolumn{3}{|c|}{ Correct Classification Rate } & $100 \%$ & & $35.7 \%$ & & $78.6 \%$ \\
\hline
\end{tabular}


3.3.Spectrochemical Mapping Using LIBS for Shooting Distance Determination on Bloody Clothing

\subsubsection{Mehtods and Materials}

\subsubsection{Sample Preparation and Collection}

White fabric (100\% cotton) was obtained from a retail store and was cut into $20 \mathrm{~cm}$ by $27 \mathrm{~cm}$ rectangles. All samples were placed in manila folders, with a white piece of copy paper on either side, and then folded in pre-labeled clean paper. The prepared samples were stored in a sealed plastic container at the laboratory until shooting and collection.

All shootings were performed at the West Virginia University Forensic and Investigative Sciences ballistics laboratory under controlled environmental conditions. Each fabric sample was hung on a self-healing shooting block using push pins and a clean manila folder support to prevent cross contamination. The distance from the muzzle of the firearm to the sample was set using a measuring tape and floor markers before each shooting. It was ensured that the individual handling and storing the samples was not the same individual performing the shootings.

A total of 25 samples were shot with a $9 \mathrm{~mm}$ pistol at known distances and used as a calibration set. The distances used for calibration purposes were contact, 6 inches, 12 inches, 24 inches, and 36 inches; each distance was repeated three times for both grey and patterned fabrics. A total of 10 unknown samples were collected; 5 pristine white samples and 5 white samples that would have blood applied back at the laboratory. The analyst was blind to the true distance of the unknown samples while performing testing.

Nitrile gloves (Fischer Scientific; $\mathrm{NH}$ ) and lab coats (Fischer Scientific; NH) were worn during sample handling, preparation, collection, and analysis. Gloves were changed between handling each new sample. Blank controls were analyzed in triplicate for the fabric type to evaluate any potential interferences.

\subsubsection{Application of Blood to White Clothing Samples}

To assess the ability of LIBS to detect GSR on soiled samples, human blood (55\% plasma:45\% red blood cells) was applied to all white known-distance fabric samples and 5 white unknown distance fabric samples, after being shot. Application of blood was conducted in a properly ventilated Nuaire Biological Safety Cabinet fume hood, with a protective layer of Wypall Wipers $®$ 
on all surfaces, back at the laboratory. A continuous spray of blood was applied to the samples, using a new Search Power Spray Units (Sirchie Acquisition Company LLC, NC), until the visible soot patterns became less discernable. Samples were then air dried in the biohazard hood, folded back in the same pre-labeled paper, and stored in the biological safety hood to protect the laboratory.

For method development of shooting distance determination using LIBS, LIBS data processing, and chemical color test methods, reagents, and measurements please refer to sections 3.2.1.3.1., $\underline{3.3 .3 .1 .}$ and $\underline{3.2 .1 .3 .3}$, respectively.

\subsubsection{Results and Discussions}

One of the most common items submitted to crime labs for distance determination is the clothing the victim was wearing during the time of the shooting. As expected, the majority of bullet would induce bleeding near the entrance and exit holes. As a result, physical characteristics that provide leading information for estimating shooting distance are masked, making visual interpretation nearly impossible. Additionally, indirect testing methods are needed, which in turn, further reduce the sensitivity of the colorimetric assays.

The LIBS method tested and validated on pristine colored clothing samples, was assessed as a potential solution to overcome the problems encountered with these non-ideal samples. Once the LIBS method was applied, the samples were subjected to colorimetric tests to follow the standard procedure of distance determination currently performed by practitioners.

\subsubsection{Firing Distance Prediction Using LIBS Analysis and Multivariate Methods}

\subsection{Chemical Maps for Shooting Distance Determinations}

As previously described in section 3.2.5.2., the distances for the purpose of a calibration set were chosen to represent three different categories of shooting distances; contact, short range (6 inches12 inches), and long range (24 inches-36 inches). After data acquisition, the spectral data was transformed into intensity versus ablation distance from bullet hole $(\mathrm{cm})$ bar graphs to visualize the distribution pattern of IGSR $(\mathrm{Pb}, \mathrm{Ba}, \mathrm{Sb})$ on the samples relative to shooting distance. For each of the calibration sets, all distances were overlaid on the same graph to evaluate the difference in chemical patterns as the shooting distance increased. As seen with the colored and patterned samples, contact samples tended to show the highest initial intensity with a rapid drop off in intensity as the ablation distance from the bullet hole increased. Figure 17 below shows a 
representation of $\mathrm{Ba}(493.4 \mathrm{~nm})$ for one of the calibration sets. Samples shot from 6 inches and 12 inches had lower initial intensities but decreased more gradually and eventually level out around $6 \mathrm{~cm}$ from the bullet hole. Longer range distance samples (24 inches and 36 inches) had consistently lower intensities for the entirety of the $10 \mathrm{~cm}$ with 24 inches having slightly higher intensities than 36 inches.

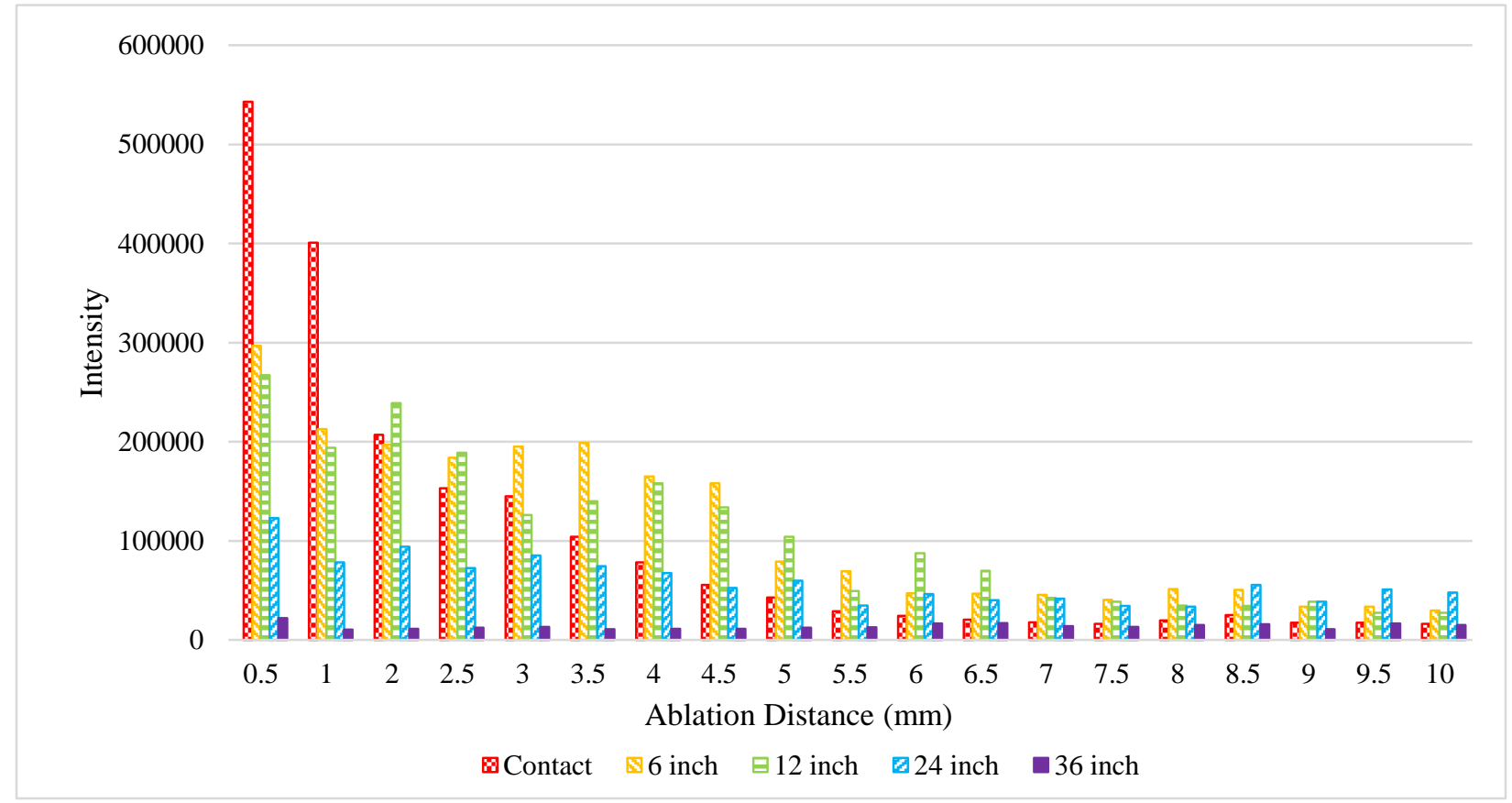

Figure 17. Bar graph representing intensity patterns for $\mathrm{Ba}(493.4 \mathrm{~nm})$ for a blood-stained fabric calibration set. From left to right, the ablation from the entrance hole begins at $0.5 \mathrm{~mm}$ and increases by $0.5 \mathrm{~mm}$ until ending at 100 $\mathrm{mm}$. The legend represents the different shooting distance used for calibration (checkered red $=$ Contact, diagonal yellow $=6$ inches, horizontal green $=12$ inches, diagonal blue $=24$ inches, solid purple $=36$ inches).

Additionally, chemical heat maps were made using obtained spectral data to provide permanent, more objective representations of GSR patterns. As the blood masked the visible soot and FDR patterns on the fabric samples, these chemical heat maps provide more accurate visualizations of lead, barium, and antimony concentrations surrounding the bullet hole. Moreover, the heat maps can be created for multiple elements associate with firearms discharge residues, unlike color tests which are limited to lead and nitrites. Figure 18 below show spatial heat maps for $\mathrm{Pb}(405.8)$ and four different calibration distances (contact, 6 inches, 12 inches, 24 inches). Much like the chemical color tests, darker colors represent a higher concentration of the element and the lighter colors represent lower concentrations of the element. Contact shots consistently had several dark rings in the center of the heat maps with a rapid decrease in darkness as the map spread out. Sixinch heat maps typically had one or two dark rings in the center with a more gradual succession to 
lighter colors. Heat maps for samples shot from 12 inches had inner rings lighter than the previous two shooting distances and a uniform decreases in darkness. Finally, 24 inch and 36 inch had uniform light colored heat maps with color barely visible for 36 inches.
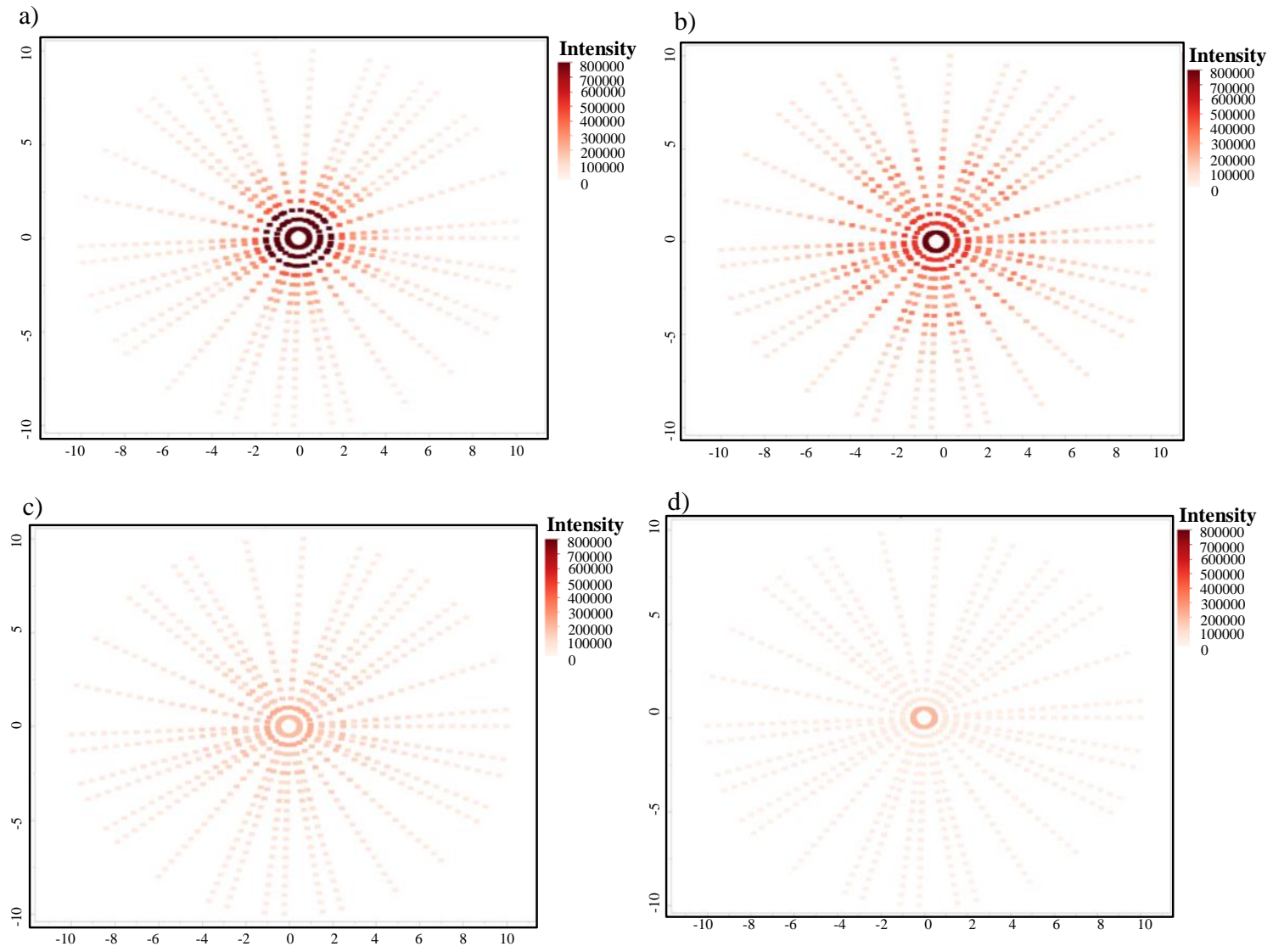

Figure 18. Heat maps of $\mathrm{Pb}(405.8 \mathrm{~nm})$ obtained from blood stained calibration set number 1. (a) contact; (b) 6 inches; (c) 12 inches; (d) 24 inches.

\subsection{Multivariate Methods}

Statistical approaches to classification of unknown distances included principal component analysis and multivariate discriminant analysis. As a first step, PCA plots were constructed using the spectral intensities found for the entirety of the $5 \mathrm{~mm}$ ablation increments; $100 \mathrm{~mm}$ total. The use of $130 \mathrm{~mm}$ was eliminated in section 3.2.5.3. when it was determined that the last $30 \mathrm{~mm}$ added little useful information for distance determination. Therefore, to avoid unnecessary noise, each sample for the blood study was ablated for a total of $100 \mathrm{~mm}$ instead of $130 \mathrm{~mm}$. Additionally, a bullet hole qualifier was included to account for the large star shaped pattern characteristic of contact shots for each calibration set. The large bullet holes allowed for some GSR to pass through the fabric instead of becoming imbedded in it, resulting in two of the contact 
samples to have lower initial intensities. To avoid the possibility of a contact shot being classified as a farther distance, the size of each bullet hole was measured. Each $5 \mathrm{~mm}$ increment had numeric outputs from the accumulated integrated areas of the emission lines for lead barium and antimony. All three replicates of calibration curve samples were used in the construction of the PCA plot. The unknowns assessed consisted of 5 pristine white clothing samples and 5 blood stained white clothing samples. When the PCA plot was constructed with all $100 \mathrm{~mm}$ of spectral data and the bullet hole qualifier, $78.7 \%$ of the variability in the data was captured.

The first two principal components, as well as the bullet hole qualifier, were used as the variable to perform discriminant analysis (Figure 19). To compensate for the small sample size associated with the study, regularized discriminant analysis was performed. The blood-stained calibration sets were used as the training sets and the unknown blood stained and pristine white unknown samples were used as the validation/test set. In practice, it is common to try to elucidate whether a firearm related incident was an accident, a suicide, or a homicide. In that case, distances are typically reported as ranges rather than a specific numerical distance. Therefore, classifications of unknowns were made using the first prediction and second prediction output by the discriminant analysis model. Six unknown samples (5 blood stained, 1 pristine) had a first prediction of 6 inches and a second prediction of 12 inches. Therefore, the reported distance for these samples would be 6 inches-12 inches with an emphasis closer to 6 inches. On the other hand, 4 unknown samples (pristine white) had a first prediction of 12 inches and a second prediction of 6 inches. For these samples, the reported shooting distance would be 6 inches- 12 inches, but with an emphasis closer to 12 inches. A misclassification occurred when the determined distance range did not include the true distance, or the determined distance range was too large. A distance range was not needed to classify an unknown sample that was a contact shot due to the characteristic star shaped pattern. 


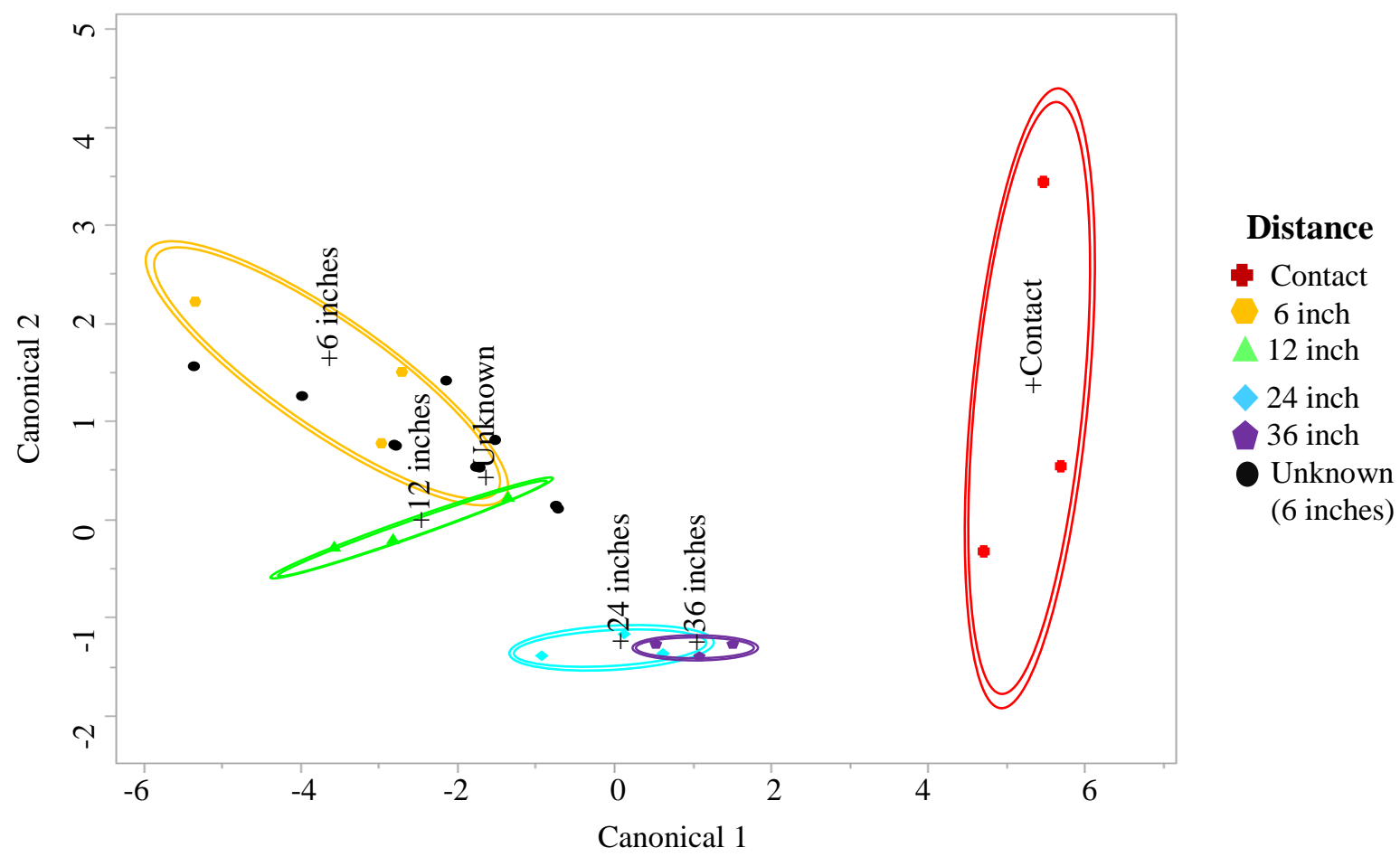

Figure 19. Canonical plot for blood stained calibration samples with blood stained unknowns $(1,2,3,4$ and 5$)$ and white unknowns $(1,2,3,4$, and 5). All unknowns have a true shooting distance of 6 inches. ( + represents the multivariate mean for each shooting distance, outer ellipses represent $95 \%$ confidence interval for each shooting distance, and inner ellipses represent a $50 \%$ contour of observations for each shooting distance.)

\subsubsection{Firing Distance Predictions using Color Tests}

\subsection{Interpretation of Physical and Visual Examination and Chemical Color Tests}

As expected, the blood on the fabric made it difficult to obtain accurate physical measurements. While soot pattern measurements were possible it was increasingly difficult to see the patterns as the shooting distance increased. Farther distances inherently have less concentrated soot patterns and with the blood it was almost impossible to determine the overall spread of the soot. A flashlight held at an oblique angle was needed to aid in counting the gun powder particles on all samples stained with blood. The pristine white unknown samples showed no difficult in taking measurements. The difficulty to obtain measurements on bloody samples illustrates one of the limitations associated with visual interpretations of evidence submitted for firearm related examinations. Additionally, measurements vary from analyst to analyst.

Based on measurements that were taken, for the calibration curve built from shooting the white textiles at different muzzle-to-fabric distances, contact shots resulted in a star-shaped rip pattern 
(about $14.5 \mathrm{~cm} \times 11.0 \mathrm{~cm}$ ) originating from the bullet hole, with very little soot and no gun powder particles being seen. The rip in the fabric was large enough to allow gun powder particles to pass through it, rather than being dispersed onto the fabric. The close-range samples consistently had larger soot patterns than the contact samples and, on average, 100-gun powder particles were visible on the fabric. No soot pattern was observed for any of the long-range shooting samples (Table 6).

Table 6

Physical measurements taken of samples before application of color tests ( $P=$ pistol, $L=l e n g t h, W=$ width, Cal=calibration, $U=$ unknown (true distance: 6 inches), $N / A=$ not visible for measurement)

\begin{tabular}{|c|c|c|c|c|c|}
\hline Sample & Distance & $\begin{array}{l}\text { Bullet hole } \\
\text { LxW (cm) }\end{array}$ & $\begin{array}{l}\text { Soot LxW } \\
(\mathrm{cm})\end{array}$ & $\begin{array}{l}\text { Gun } \\
\text { Powder } \\
\text { LxW (cm) }\end{array}$ & $\begin{array}{l}\text { Gun } \\
\text { Particle } \\
\text { Count } \\
\end{array}$ \\
\hline Cal Blood Set $1(P)$ & contact & $0.6 \times 0.5$ & $3.2 \times 3.4$ & $4.8 \times 8.1$ & 16 \\
\hline Cal Blood Set $1(P)$ & 6 in & $0.9 \times 1.0$ & $5.3 \times 6.4$ & $6.3 \times 8.3$ & 89 \\
\hline Cal Blood Set $1(P)$ & 12 in & $0.6 \times 0.9$ & $4.1 \times 4.3$ & $8.2 \times 6.3$ & 91 \\
\hline Cal Blood Set $1(P)$ & $24 \mathrm{in}$ & $0.9 \times 0.8$ & N/A & $8.1 \times 9.6$ & 154 \\
\hline Cal Blood Set $1(P)$ & 36 in & $0.8 \times 0.7$ & N/A & $10.9 \times 9.2$ & 21 \\
\hline Cal Blood Set $2(P)$ & contact & $0.8 \times 0.8$ & $4.1 \times 3.2$ & $12.3 \times 6.1$ & 4 \\
\hline Cal Blood Set $2(P)$ & 6 in & $0.8 \times 0.8$ & $3.2 \times 2.9$ & $8.0 \times 8.3$ & 87 \\
\hline Cal Blood Set $2(P)$ & 12 in & $0.7 \times 0.8$ & $4.6 \times 4.5$ & $7.7 \times 7.9$ & 123 \\
\hline Cal Blood Set $2(P)$ & $24 \mathrm{in}$ & $0.7 \times 0.9$ & N/A & $10.4 \times 7.7$ & 111 \\
\hline Cal Blood Set $2(P)$ & 36 in & $0.7 \times 0.8$ & N/A & $6.1 \times 10.8$ & 16 \\
\hline Cal Blood Set $3(P)$ & contact & $0.7 \times 0.6$ & $4.0 \times 3.8$ & N/A & 0 \\
\hline Cal Blood Set $3(P)$ & 6 in & $0.6 \times 0.8$ & $5.2 \times 4.8$ & $8.0 \times 6.1$ & 94 \\
\hline Cal Blood Set $3(P)$ & $12 \mathrm{in}$ & $0.8 \times 0.7$ & $4.2 \times 4.1$ & $10.3 \times 6.1$ & 108 \\
\hline Cal Blood Set $3(P)$ & $24 \mathrm{in}$ & $0.7 \times 0.7$ & N/A & $6.6 \times 8.2$ & 73 \\
\hline Cal Blood Set $3(P)$ & 36 in & $0.6 \times 0.8$ & N/A & $8.7 \times 7.0$ & 15 \\
\hline Blood & $\mathrm{U} 1$ & $0.7 \times 0.8$ & $5.2 \times 5.3$ & $9.5 \times 7.0$ & 80 \\
\hline Blood & $\mathrm{U} 2$ & $0.8 \times 0.9$ & $4.7 \times 4.8$ & $10.2 \times 8.3$ & 130 \\
\hline Blood & U 3 & $0.8 \times 0.9$ & $4.5 \times 5.8$ & $8.5 \times 5.1$ & 55 \\
\hline Blood & $\mathrm{U} 4$ & $0.8 \times 0.9$ & $6.3 \times 5.4$ & $8.4 \times 5.6$ & 48 \\
\hline Blood & $\mathrm{U} 5$ & $0.8 \times 0.9$ & $5.1 \times 5.0$ & $4.6 \times 6.8$ & 76 \\
\hline Non-blood & U 6 & $0.8 \times 0.8$ & $5.0 \times 5.9$ & $7.2 \times 4.9$ & 93 \\
\hline Non-blood & U 7 & $0.8 \times 0.9$ & $5.1 \times 7.1$ & $10.6 \times 5.2$ & 95 \\
\hline Non-blood & U 8 & $0.9 \times 0.8$ & $4.2 \times 5.7$ & $8.2 \times 8.2$ & 169 \\
\hline Non-blood & U 9 & $0.8 \times 0.8$ & $5.8 \times 5.2$ & $10.5 \times 5.0$ & 116 \\
\hline Non-blood & U10 & $0.8 \times 0.8$ & $5.5 \times 5.0$ & $10.1 \times 9.2$ & 134 \\
\hline
\end{tabular}

All 25 samples in this study were subjected to both the modified Griess test and the sodium rhodizonate test (Table 7). Positive controls were performed on the corners of prepared Griess test sheets prior to analysis for each of the samples. It should also be noted that LIBS ablations did not 
interfere with the macro distribution pattern of gunshot residue on the fabric samples. The laser moving quickly across the surface created a microscopic ablation line on the surface of the sample and did not disrupt surrounding soot or gunpowder particles. Therefore, there was no disruption in the modified Griess test patterns produced.

Table 7

Physical measurements taken after modified Griess and sodium rhodizonate tests $(P=$ pistol, $R=$ revolver, $L=$ length, $W=$ width, Cal=calibration, $U=$ unknown (true distance: 6 inches), N/A= not visible for measurement)

\begin{tabular}{|c|c|c|c|c|c|}
\hline Sample & Distance & $\begin{array}{l}\text { Area Griess } \\
\text { Color } \\
\left(\mathrm{cm}^{2}\right) \\
\end{array}$ & $\begin{array}{l}\text { Area Griess } \\
\text { Particle } \\
\left(\mathrm{cm}^{2}\right) \\
\end{array}$ & $\begin{array}{l}\text { Area Sodium } \\
\text { Rhodizonate } \\
\text { Outer }\left(\mathbf{c m}^{2}\right) \\
\end{array}$ & $\begin{array}{l}\text { Area Sodium } \\
\text { Rhodizonate } \\
\text { Inner }\left(\mathrm{cm}^{2}\right) \\
\end{array}$ \\
\hline Cal Blood Set $1(P)$ & contact & 11.5 & 5.5 & 107.5 & 34.1 \\
\hline Cal Blood Set $1(P)$ & 6 in & 38.4 & 60.8 & 72.2 & 9.5 \\
\hline Cal Blood Set $1(P)$ & 12 in & 55.1 & 54.8 & N/A & N/A \\
\hline Cal Blood Set $1(P)$ & $24 \mathrm{in}$ & 44.5 & 110.9 & N/A & N/A \\
\hline Cal Blood Set $1(P)$ & 36 in & N/A & 73.1 & N/A & N/A \\
\hline Cal Blood Set $2(P)$ & contact & 12.2 & 47.2 & 41.3 & 16.3 \\
\hline Cal Blood Set $2(P)$ & 6 in & 40.3 & 51.7 & 42.7 & 10.6 \\
\hline Cal Blood Set $2(P)$ & $12 \mathrm{in}$ & 62.1 & 137.8 & N/A & N/A \\
\hline Cal Blood Set $2(P)$ & $24 \mathrm{in}$ & 24.1 & 41.4 & N/A & N/A \\
\hline Cal Blood Set $2(P)$ & 36 in & N/A & 80.2 & N/A & N/A \\
\hline Cal Blood Set $3(P)$ & contact & 12.5 & N/A & 30.4 & 17.7 \\
\hline Cal Blood Set $3(P)$ & 6 in & 62.7 & 60.2 & 50.5 & 18.9 \\
\hline Cal Blood Set $3(P)$ & 12 in & 82.5 & 72.3 & N/A & 6.2 \\
\hline Cal Blood Set $3(P)$ & $24 \mathrm{in}$ & N/A & 53.6 & N/A & N/A \\
\hline Cal Blood Set $3(P)$ & 36 in & N/A & 62.8 & N/A & N/A \\
\hline Blood & $\mathrm{U} 1$ & 35.2 & 62.1 & N/A & 17.4 \\
\hline Blood & $\mathrm{U} 2$ & 34.8 & 57.8 & 56.5 & 20.1 \\
\hline Blood & U 3 & 29.9 & 51.9 & N/A & 19.8 \\
\hline Blood & $\mathrm{U} 4$ & 28.7 & 22.1 & 59.9 & 23.3 \\
\hline Blood & U 5 & 59.8 & 61.2 & N/A & 70.0 \\
\hline Non-blood & U 6 & 38.8 & 63.5 & 50.2 & 14.9 \\
\hline Non-blood & U 7 & 33.1 & 55.7 & 65.7 & 24.0 \\
\hline Non-blood & U 8 & 27.3 & 83.6 & 103.8 & 20.6 \\
\hline Non-blood & U 9 & 30.4 & 45.5 & 51.5 & 15.6 \\
\hline Non-blood & $\mathrm{U} 10$ & 21.2 & 29.3 & 77.0 & 25.1 \\
\hline
\end{tabular}

The modified Griess test proved to enhance the gunshot residue distributions that were masked by the blood applied to the clothing. The calibration curve samples showed consistent Griess test results for all replicate samples (Figure 20). The contact shots resulted in a faint orange circle concentrated tightly around the bullet hole. The color is faint because of the star shaped pattern previously mentioned. The 6 inch and 12-inch shots resulted in a more dispersed orange color, 
with the darkest part being at the center of the bullet hole. A dark orange color where gunpowder particles were located on the fabric was also seen at these distances. For the long-range shooting distance, no overall orange color was observed, only slight residues from gun powder particles.
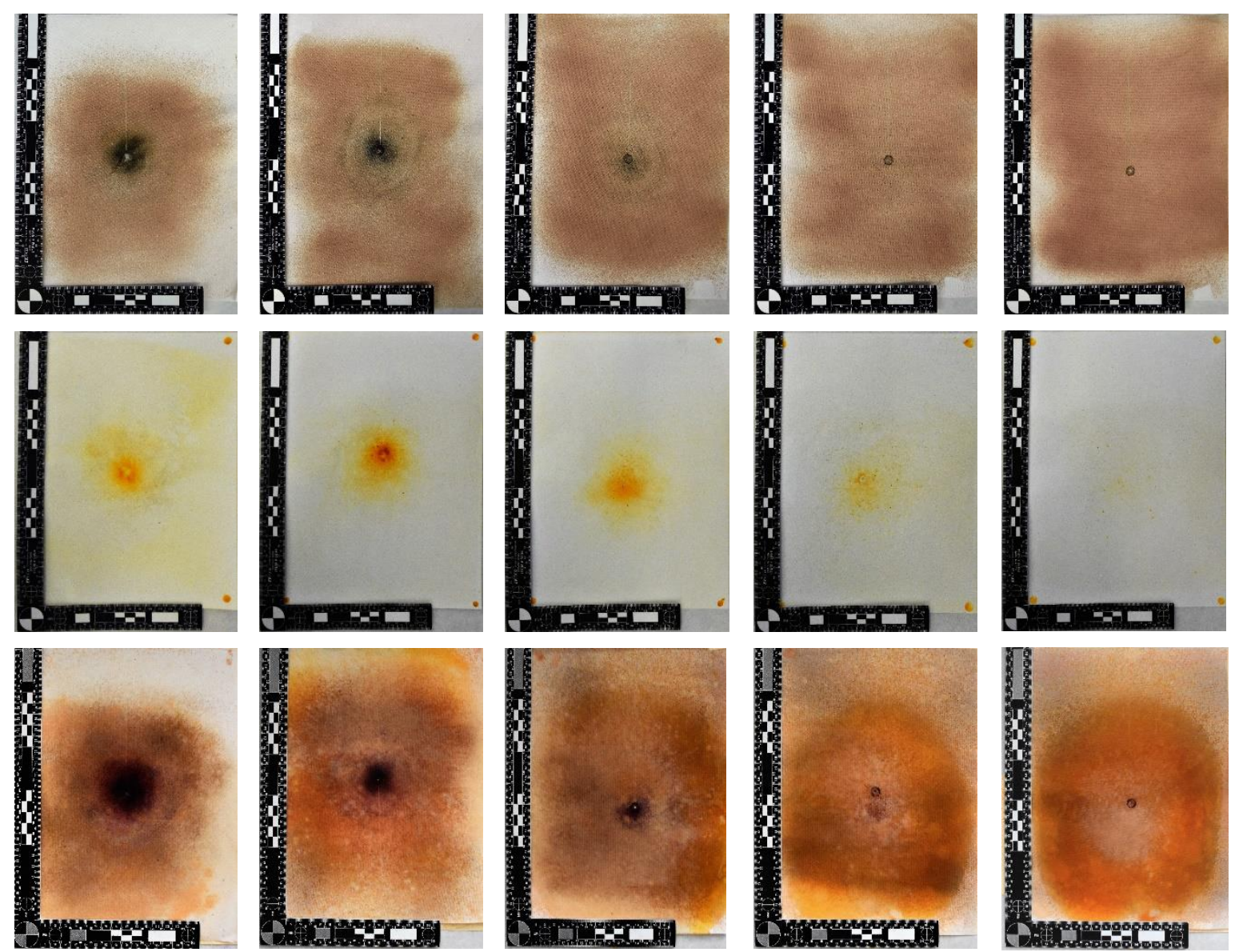

Figure 20. Photographs of original samples (top) and results after the modified Griess test (middle) was performed on photographic paper and the sodium rhodizonate test (bottom) was performed on the sample (scale: $280 \mathrm{~mm} \times 150$ $\mathrm{mm}$ ). Blood-stained Calibration set (from left to right: contact, 6 inches, 12 inches, 24 inches, 36 inches).

The sodium rhodizonate test was not sensitive enough to fully detect the low concentration of lead in specific primer used in for this study. Purple colors were only visible for samples shot at close ranges. Furthermore, the blood masked the purple color making it difficult to accurately measure the area of color produced (Figure 20). Therefore, the shooting distance predictions using color tests heavily relied on the measurements taken for the modified Griess test as opposed to the sodium rhodizonate test. 


\subsubsection{True Shooting Distance: Assessment of Accuracy of the Methods}

In practice, a range of shooting distances is typically reported rather than a specific shooting distance. Determination of a contact, close range (6 inches-12 inches), or long range (24 inches36 inches) shooting becomes important in reconstructing the events of a crime scene. Therefore, in this study, all unknown samples were reported as a range of distances. A proper classification was reported when the true shooting distance falls into the correct 1-increment distance range (contact, 6 inches-12 inches, 12 inches-24 inches, 24 inches-36 inches) and an incorrect classification was reported when the range falls outside the true distance or the range is larger than one increment. The proposed LIBS and statistical methods method correctly classified 100\% of the 10 unknown shooting distance samples analyzed in this study. In contrast, 50\% of unknown samples were correctly classified when relying on physical measurements and only $30 \%$ were

correctly classified when considering color test measurements. Table 8 shows a breakdown of classifications results for each sample based on the technique used. 


\section{Table 8}

Summary table of distance range classifications for the LIBS method, physical measurements, modified Griess and sodium rhodizonate color tests $($ Bold \#= model's first prediction, Non-bold \#= model's second prediction)

\begin{tabular}{|c|c|c|c|c|c|c|c|}
\hline $\begin{array}{l}\text { Unknown } \\
\text { Sample Name }\end{array}$ & $\begin{array}{l}\text { LIBS/Discriminant } \\
\text { Analysis Distance } \\
\text { Range } \\
\text { Classification }\end{array}$ & $\begin{array}{l}\text { Actual } \\
\text { Distance }\end{array}$ & $\begin{array}{l}\text { Correct Distance } \\
\text { Range by LIBS/ } \\
\text { Discriminant } \\
\text { analysis? }\end{array}$ & $\begin{array}{l}\text { Visual/Physical } \\
\text { Distance Range } \\
\text { Classification }\end{array}$ & $\begin{array}{l}\text { Correct Distance } \\
\text { Range by } \\
\text { Visual/Physical? }\end{array}$ & $\begin{array}{l}\text { Color Tests } \\
\text { Distance Range } \\
\text { Classification }\end{array}$ & $\begin{array}{l}\text { Correct } \\
\text { Distance } \\
\text { Range by } \\
\text { Color Tests } \\
\end{array}$ \\
\hline $1 \operatorname{Blood}(P)$ & 6-12 inches & 6 inches & Yes & 6-12 inches & Yes & 6-12 inches & Yes \\
\hline 2 Blood $(P)$ & 6-12 inches & 6 inches & Yes & $6-12$ inches & Yes & 6-12 inches & Yes \\
\hline $3 \operatorname{Blood}(P)$ & 6-12 inches & 6 inches & Yes & 6-12 inches & Yes & Contact-12 inches & RTL \\
\hline $4 \operatorname{Blood}(P)$ & 6-12 inches & 6 inches & Yes & 6-12 inches & Yes & Contact-24 inches & RTL \\
\hline $5 \operatorname{Blood}(P)$ & 6-12 inches & 6 inches & Yes & Contact- 6 inches & No & Contact-24 inches & RTL \\
\hline 6 Non-Blood $(P)$ & 6-12 inches & 6 inches & Yes & 6-24 inches & RTL & Contact- 12 inches & RTL \\
\hline 7 Non-Blood $(P)$ & 6-12 inches & 6 inches & Yes & 12-24 inches & No & 6-24 inches & RTL \\
\hline 8 Non-Blood $(P)$ & 6-12 inches & 6 inches & Yes & 6-12 inches & Yes & Contact-24 inches & RTL \\
\hline 9 Non-Blood $(P)$ & 6-12 inches & 6 inches & Yes & 12-24 inches & No & 6-12 inches & Yes \\
\hline $\begin{array}{l}10 \text { Non-Blood } \\
(P)\end{array}$ & 6-12 inches & 6 inches & Yes & 12-24 inches & No & 6-24 inches & RTL \\
\hline $\begin{array}{l}\text { Correct Classi } \\
\text { Rate }\end{array}$ & ation & & $100 \%$ & & $50 \%$ & & $30 \%$ \\
\hline
\end{tabular}




\subsection{Conclusions}

The challenges and limitations associated with the current methods for shooting distance determination emphasize the need for a versatile analytical tool that will enhance the scientific reliability of the detection, observation, and interpretation of GSR patterns on substrates of interest commonly encountered at a crime scene. The purpose of this study was to develop a more objective, reliable, sensitive, and superior approach to determining the distance from which a firearm was discharged. The use of laser-induced breakdown spectroscopy in this discipline has shown to have advantages over current chemical methods, including:

Ease of sample preparation and analysis: The LIBS analytical method does not require any additional preparation of the sample. Additionally, unlike color tests, LIBS does not require the use of fume hood, proper disposal of hazardous waste, preparation of fresh reagents due to their instability, and photographic documentation between the steps of color development.

Minimal destruction of substrate: The substrate of interest is negligibly damaged during the experimental process, which allows for reanalysis or subsequent analysis by other methods if necessary.

Selectivity and multi-elemental detection capabilities: LIBS is capable of detecting multiple elemental emission lines associated with firearm discharge residues (several atomic and ionic emission lines of $\mathrm{Pb}, \mathrm{Ba}, \mathrm{Sb}, \mathrm{Al}, \mathrm{Cu}$ ) simultaneously, improving selectivity and confidence in the results.

Superior sensitivity: LIBS is able to detect elements associated with GSR in the low ppm concentrations. This is essential with the advancement of more environmentally friendly primers containing reduced concentrations of heavy metals. Additionally, LIBS is capable of detecting multiple atomic and ionic emission lines for each element, increasing the certainty of its presence while decreasing the rate of false positives.

More objective interpretation of GSR patterns: LIBS has the capability of capturing spatial information which allows for the creation of permanent and objective chemical mapping of GSR patterns on clothing samples and other substrates and the use of statistical tools for prediction of distance. 
The application of this study is anticipated to aid in crime scene reconstruction when a firearm is involved in a criminal event and offer more reliable investigative information and defensible results in court. 


\section{CHAPTER 2: THE DEVELOPMENT OF A FAST CHEMICAL MAPPING METHOD FOR THE IDENTIFICATION OF ENTRANCE HOLES}

\subsection{Overview of Project}

This chapter proposes the application of Laser-Induced Breakdown Spectroscopy (LIBS) as a novel, practical approach to identifying bullet holes in multiple substrates and surfaces in firearm related incidents. The substrates chosen are representative of common surfaces at crime scenes that are large, non-movable, and difficult to perform GSR detection on. A total of 21 substrates of varying material (glass, wood, and drywall) were shot from a close distance to simulate the scene of a firearm related crime. The GSR surrounding the bullet hole was transferred to an adhesive sheet to eliminate the need to transfer the substrates back to the laboratory. The adhesive samples were then analyzed by LIBS using a rapid spectral mapping method. Spatial distributions of inorganic gunshot residues, including lead, barium, and antimony were used to determine if the hole in the substrate was created by a bullet. The adhesive transfer method proved to be a suitable practice for collecting gunshot residue off of the multiple substrates. The substrates showed differing difficulties of application, however, overall the method allowed for GSR to be detected on all adhesive samples collected. Figure 21 shows a detailed layout of the number of samples, objectives, and specific analyses performed for each sample set. 


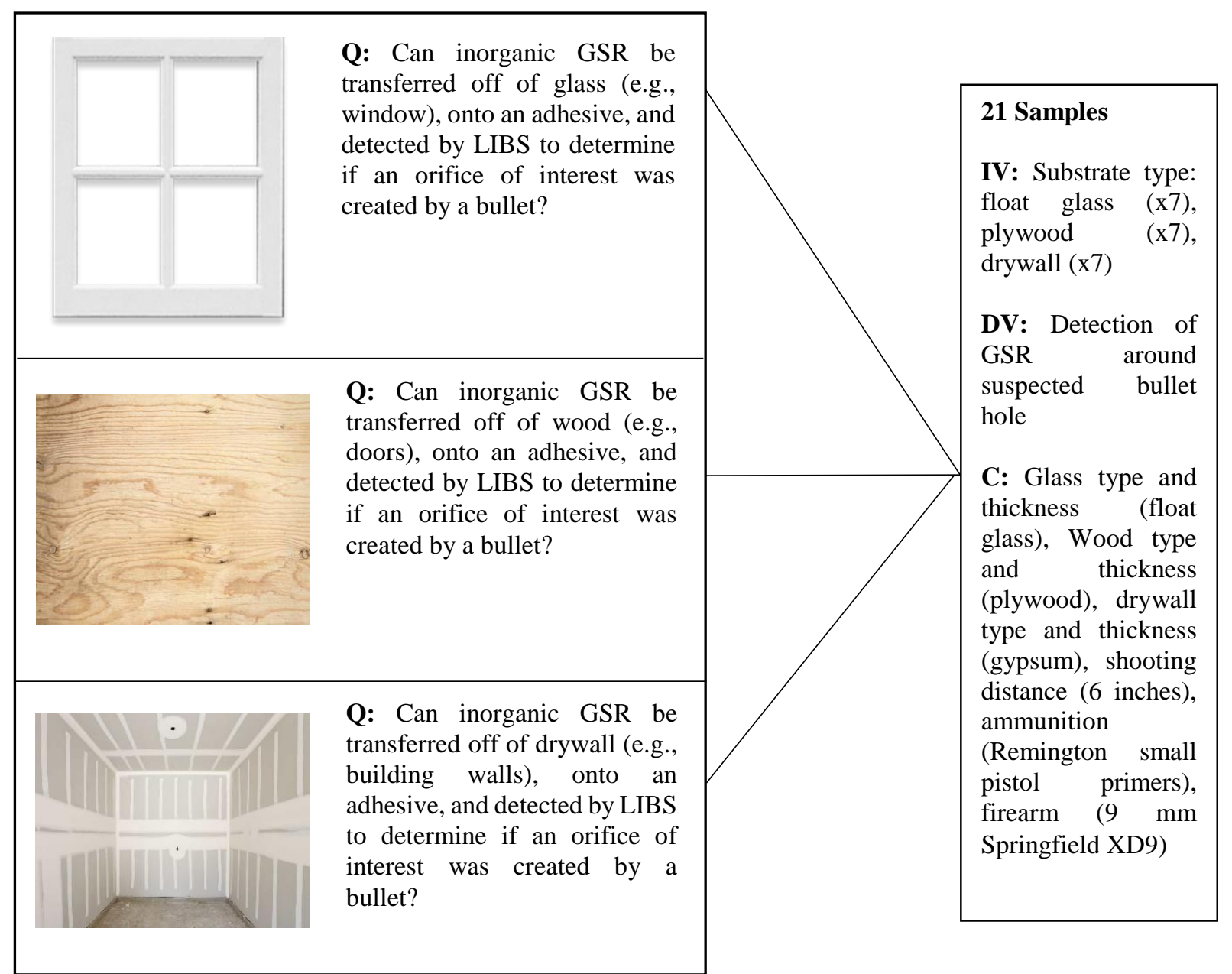

Figure 21. Experimental design for bullet hole identification by analysis of IGSR using LIBS including substrate types, variables, and number of samples for each substrate. $(\mathrm{IV}=$ independent variable, $\mathrm{DV}=$ dependent variable, $\mathrm{C}=$ constant variable, $\mathrm{Q}=$ question)

\subsection{Methods and Materials}

\subsubsection{Firearms and Ammunitions}

A $9 \mathrm{~mm}$ Springfield XD9 firearm (manufactured in Croatia) was used for all sample shooting and collection. Starline Brass $9 \mathrm{~mm}$ Luger cartridge cases reloaded with Remington 11/2 small pistol primers, Winchester 231 grain powder, and Speer $9 \mathrm{~mm}$ Luger total metal jacket (TMJ) bullets were used for sample shooting. Total metal jacketed bullets were chosen to ensure the majority of lead seen in a LIBS spectra was coming from the primer as opposed to the bullet itself.

\subsubsection{Substrate Preparation and Collection}

Multiple substrates including, plywood, float glass, and dry wall were obtained from a local retail store. Each substrate was cut into $38 \mathrm{~cm}$ by $38 \mathrm{~cm}$ squares. All samples were folded into clean, 
white, butcher paper and stored in the laboratory to avoid contamination until sample shooting. A $182 \mathrm{~cm}$ tall wooden structure, with a $38 \mathrm{~cm}$ by $38 \mathrm{~cm}$ inset, was built to safely secure each substrate during shooting (Figure 22).

Three different transfer substrates (Handiprint Adhesive, SIRCHIE Tape Pads, Whatman 42 paper) were tested on $100 \%$ white cotton samples shot from a close range to assess ease of application, retention of GSR, and possible elemental interferences. Each substrate was applied to the GSR samples, analyzed using the LIBS, stored on a cover sheet, and analyzed by LIBS again once removed from the cover sheet.

All shootings were performed at the West Virginia University Forensic and Investigative Sciences ballistics laboratory under controlled environmental conditions. Before shooting, the walls, ceilings, and floors of the shooting range were covered in layers of plastic tarp to avoid stray pieces of wood, glass, and drywall damaging or contaminating the range. Each substrate sample was individually secured to the wooden structure using Irwin Spring Clamps when being fired at. The distance from the muzzle of the firearm to the sample was set using a measuring tape and floor markers before each shooting. It was ensured that the person handling and storing the samples was not the same individual performing the shootings.

Each sample was shot from a distance of 6 inches to ensure ample GSR deposition for analysis. A total of 21 samples were collected for this study; 7 drywall, 7 glass, and 7 wood. All glass samples were prepped on one side with a layer of non-interfering adhesive to attempt to maintain the integrity of the sample once shot. The non-adhesive side of the glass was the entrance surface. To collect the GSR from the substrates, an 8 inch x 8 inch Handiprint Adhesive (CSI Forensic Supply, CA) square was directly applied to the entrance side of the substrate immediately after shooting. The center of the bullet hole was marked on the back of the adhesive to aid in locating the area of interest when performing LIBS analysis. The adhesive was then peeled off the substrate and stored on a cover sheet (CSI Forensic Supply, CA). The adhesive/cover sheet was placed in a manila folder and stored in folded, pre-labeled, clean butcher paper.

Nitrile gloves (Fischer Scientific; NH) and lab coats (Fischer Scientific; NH) were worn during sample handling, preparation, collection, and analysis. Gloves were changed between handling each new sample. Blank controls were analyzed in triplicate for the Handiprint adhesive, adhesive cover sheet, plywood, float glass, and drywall to evaluate any potential interferences. 


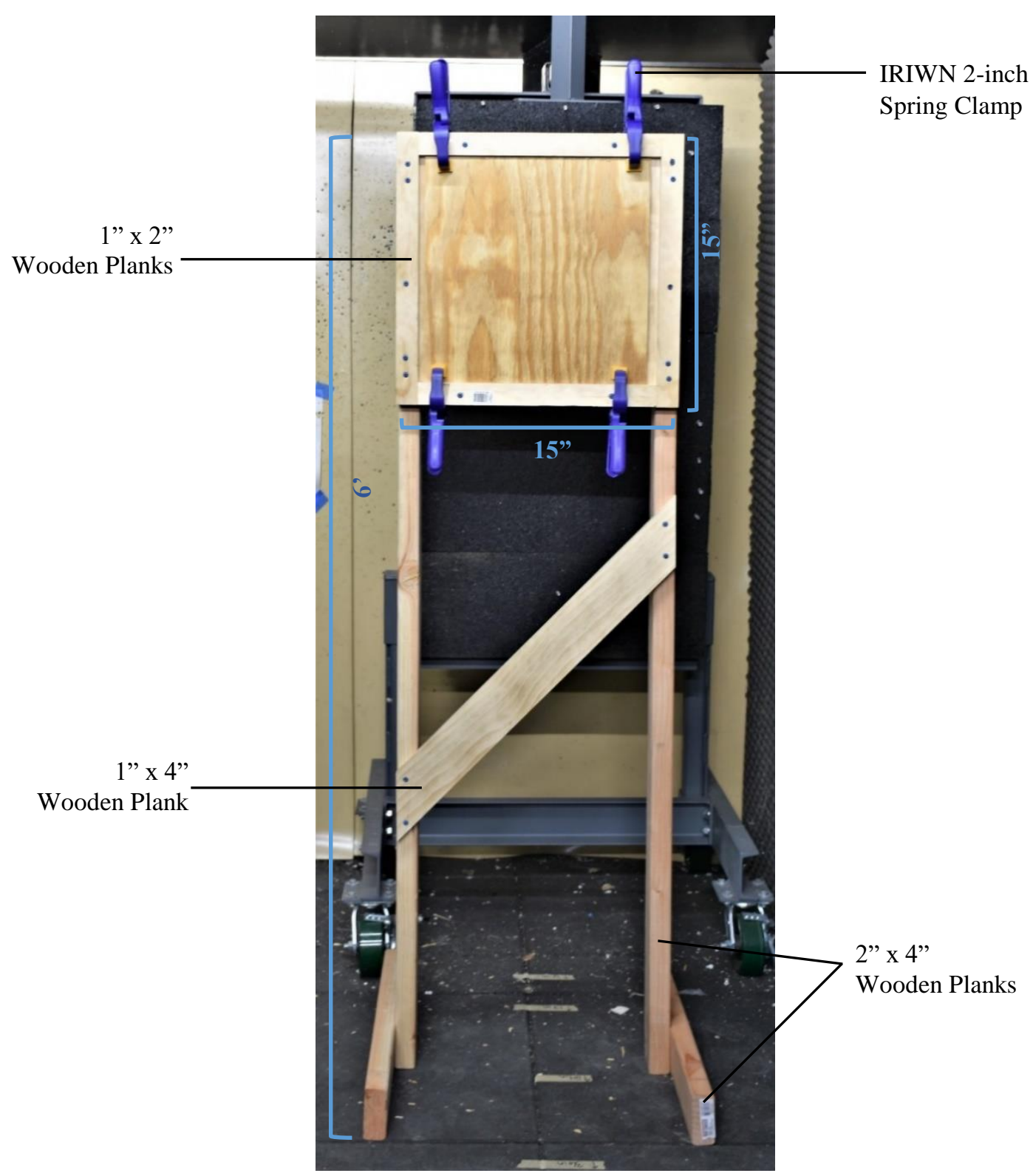

Figure 22. Custom-made wooden structure for securing substrate samples while shooting.

\subsubsection{Experimental Set $U p$}

The experimental layout was designed to implement a rapid and more efficient method for the identification of bullet holes in large, non-moveable surfaces that are commonly encountered at crime scenes. The plywood, float glass, and drywall substrates are meant to represent ordinary surfaces including architectural windows, wooden doors/structures, and architectural walls. When developing the experimental design factors time of analysis and sample destruction were also considered. The developed method of analysis takes less than 30 minutes per sample and allows 
for subsequent analysis using the aforementioned LIBS method for shooting distance determination, if necessary. The LIBS method moves the laser fast across a small area of the sample which interacts with a negligible amount of sample and keeps the integrity of the overall macro-pattern of GSR intact.

\subsubsection{LIBS Instrumentation, Parameters, and Method}

Please refer to section $\underline{\text { 3.2.1.3.1 }}$ for details on LIBS instrumentation used for data acquisition.

The adhesives containing GSR were secured to a custom-made ablation stage that sits on top of the cell in the ablation chamber, with a non-interfering double-sided adhesive tape. The stage was once again covered with benchkote paper (Fisher Scientific, NH) to prevent contamination from the metal surface. Each adhesive sample had three ablation patterns performed to create a "mapping" of the GSR present around the suspected bullet hole (Figure 23). A $10 \mathrm{~mm}$ x $10 \mathrm{~mm}$ square of 45 spots (50 $\mu \mathrm{m}$ spot size) was ablated directly around the entrance orifice. The laser was fired with an energy of $100 \%$, a repetition of $10 \mathrm{~Hz}$, and a stage velocity of $1 \mathrm{~mm} / \mathrm{s}$. A signal was accumulated for two shots at every spot. An additional $60 \mathrm{~mm}$ x $60 \mathrm{~mm}$ square pattern of a total 180 spots (50 um spot size) was created $25 \mathrm{~mm}$ away from the bullet hole on all sides. The laser was once again fired with an energy of $100 \%$, a repetition rate of $10 \mathrm{~Hz}$, a stage velocity of $1 \mathrm{~mm} / \mathrm{s}$ and an accumulation of 2 shots per spot. The last pattern was created on the corner of the fabric to serve as a negative control of GSR. A smaller $5 \mathrm{~mm}$ x $5 \mathrm{~mm}$ square of 20 spots (50 um spots) was ablated with the same laser parameters previously mentioned. Tables 9-11 below summarize the LIBS parameters used for each pattern.

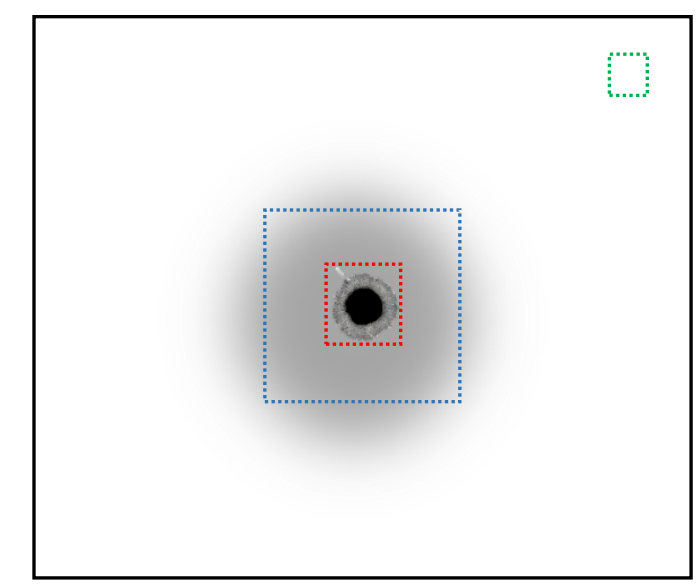

Figure 23. Diagram of ablation patterns created for bullet hole identification. Red square: 10 $\mathrm{mm}$ x $10 \mathrm{~mm}$ inner bullet hole pattern, Blue Square: $60 \mathrm{~mm}$ x $60 \mathrm{~mm}$ outer bullet hole pattern, Green Square: $5 \mathrm{~mm}$ x $5 \mathrm{~mm}$ blank pattern. 
Table 9

LIBS Parameters of Inner Bullet Hole Ablation Pattern used for Bullet Hole Identification

\begin{tabular}{ll}
\hline Parameter & Measure \\
\hline Ablation Pattern & $10 \mathrm{~mm}$ x $10 \mathrm{~mm}$ square \\
Spot Size & $50 \mathrm{um}$ \\
Number of spots per $10 \mathrm{~mm}$ side & 15 \\
Number of shots per spot & 2 \\
Pulse repetition rate & $10 \mathrm{~Hz}$ \\
Pulse Energy & $100 \%$ \\
Stage Velocity & $1 \mathrm{~mm} / \mathrm{s}$ \\
Gate Delay & 0.5
\end{tabular}

Table 10

LIBS Parameters of Outer Bullet Hole Ablation Pattern used for Bullet Hole Identification

\begin{tabular}{ll}
\hline Parameter & Measure \\
\hline Ablation Pattern & $60 \mathrm{~mm}$ x $60 \mathrm{~mm}$ square \\
Spot Size & $50 \mathrm{um}$ \\
Number of spots per $60 \mathrm{~mm}$ side & 45 \\
Number of shots per spot & 2 \\
Pulse repetition rate & $10 \mathrm{~Hz}$ \\
Pulse Energy & $100 \%$ \\
Stage Velocity & $1 \mathrm{~mm} / \mathrm{s}$ \\
Gate Delay & 0.5
\end{tabular}

Table 11

LIBS Parameters of Blank Ablation Pattern used for Bullet Hole Identification

\begin{tabular}{ll}
\hline Parameter & Measure \\
\hline Ablation Pattern & $5 \mathrm{~mm}$ x $5 \mathrm{~mm}$ square \\
Spot Size & $50 \mathrm{um}$ \\
Number of spots per $5 \mathrm{~mm}$ side & 5 \\
Number of shots per spot & 2 \\
Pulse repetition rate & 10 \\
Pulse Energy & $100 \%$ \\
Stage Velocity & $1 \mathrm{~mm} / \mathrm{s}$ \\
Gate Delay & 0.5
\end{tabular}




\subsubsection{LIBS Data Processing and Analysis}

Please refer to section $\underline{3.2 .1 .3 .2}$ for elements of interest and software used to process LIBS data obtained.

\subsection{Results and Discussion}

The identification of a bullet hole, or the differentiation between an entrance and exit bullet hole, is important during crime scene investigation but not always an easy task. Dark, blood-stained, or partially decomposed textiles can mask physical characteristics that make identifying a bullet hole simple. Additionally, other materials and substrates may make it impossible to discern whether the hole was created by a bullet or other type of destructive tool.

The main goal of this study was to assess an innovative method for identification of bullet holes in materials found at crime scenes that cannot be directly analyzed by conventional tests. The method implemented the use of an adhesive to transfer GSR from the target surface. The adhesive was then rapidly analyzed by LIBS to determine whether or not a suspected entrance hole was created by a bullet or some other penetrating source.

\subsubsection{Assessment of Adhesive Selection for Transfer of Gunshot Residues}

The three materials assessed for transferring GSR were chosen because they are readily available and already used in other forensic disciplines including trace evidence and footwear analysis. The HandiPrint and SIRCHIE Tape pads were white and transparent in color, respectively, and had one side with an adhesive. The Whatman \#42 paper was a white filter paper with no adhesive. The ability of each adhesive to pick up GSR was first tested on white cotton textiles that were previously shot from 6 inches away with a $9 \mathrm{~mm}$ Springfield hand gun. Each adhesive was first applied to bullet hole on the textile with uniform pressure and then removed to test ease of application and removal from a sample. All three transfer materials were equally simple to apply to the textile sample. Whatman \#42 was the easiest to remove from the textile due to its lack of adhesive. However, once removed, it appeared as if minimal residues were transferred to the filter paper. The HandiPrint material gave some resistance when removing because of its adhesion properties, but was overall simple to remove. Residues in the form of soot and gunpowder particles were clearly visible on the white adhesive once fully removed. Finally, the SIRCHIE Tape Pads

required the most effort to remove from the sample after application. Pulling the tape pad off 
caused stretching and some folding of the adhesive onto itself. Once removed, gunpowder particles were visible through the transparent film and some soot was detectable.

Each transfer medium was then analyzed by LIBS to determine if gunshot residues were transferred from the textile and evaluate background levels and potential interferences. A series of 5 vertical $5 \mathrm{~mm}$ lines were ablated on each transfer medium. The Whatman \#42 paper showed the lowest intensities of lead, barium, and antimony. The two adhesive transfer mediums, HandiPrint and SIRCHIE tape pads, showed similar elemental intensities with HandiPrint having slightly lower recoveries. A cover sheet was then placed on each material to preserve the transferred residues. Once the coversheet was removed, comparative LIBS spectra were obtained to assess ability of each material to retain GSR after storage (Figure 24). The Whatman \#42 paper was not sticky, and therefore, the coversheet tended to move around the sample easily displacing the GSR present. This resulted in elemental intensities that were below limits of detection. Both adhesive materials were protected by their cover sheets and show less loss of GSR compared to the Whatman \#42 paper. The HandiPrint adhesive showed the greatest retention of GSR with LIBS spectra being most similar before and after a cover sheet was applied.

Triplicate background samples of each transfer medium, and their respective coversheets, were also taken to assess the presence of potential interferences with emission lines corresponding to elements of interest (Figure 25). All background spectra collected confirmed that all materials used in this part of the study did not interfere with elements associated with GSR. Based on applicability results of each transfer medium, the HandiPrint adhesive was selected as the sampling medium of choice. 

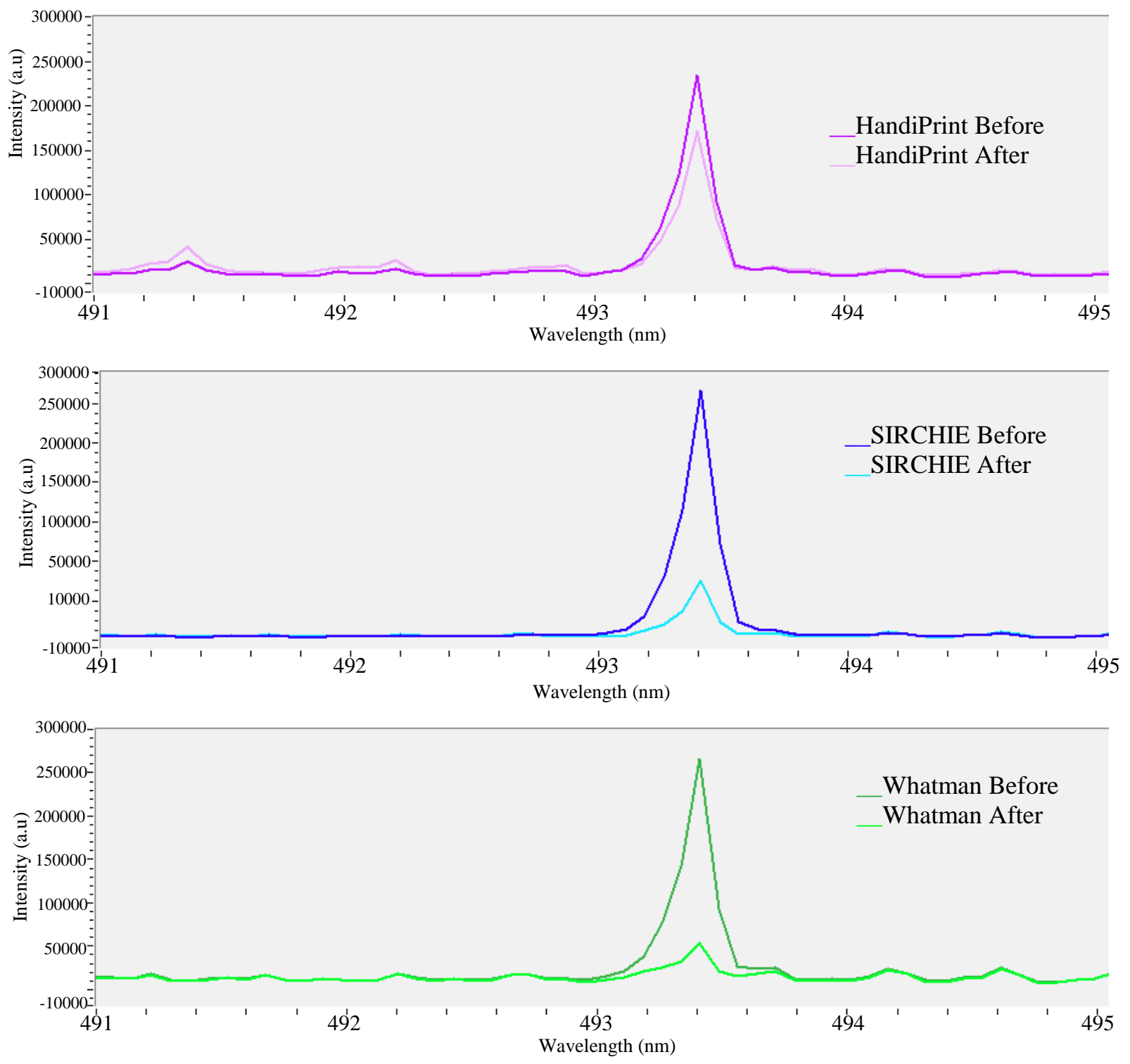

Figure 24. LIBS spectra of Ba $493.4 \mathrm{~nm}$ detected from transferred IGSR before and after application of coversheet. Top: HandiPrint Adhesive, Middle: SIRCHIE Tape Pad Adhesive, and Bottom: Whatman \#42 Paper. 


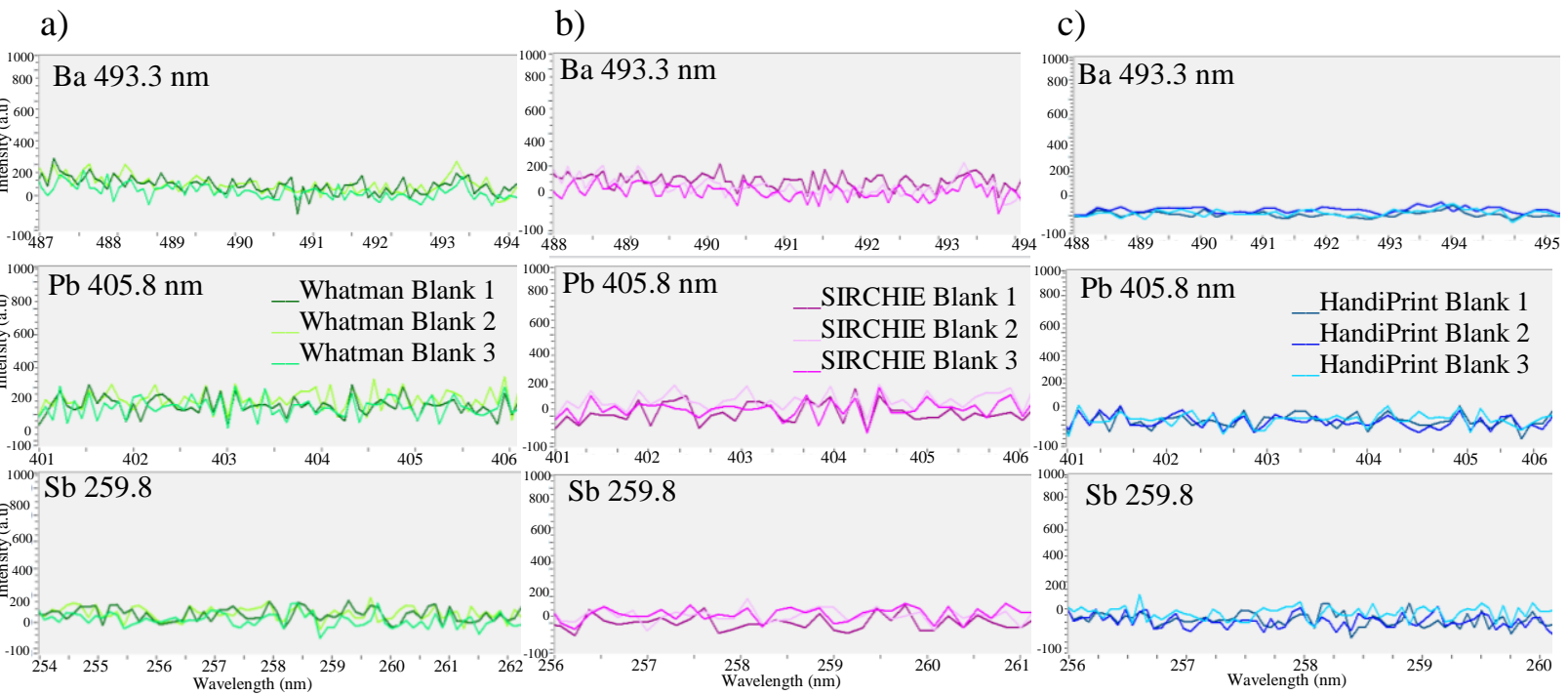

Figure 25. LIBS background spectra for a) Whatman \#42 Paper b) SIRCHIE Tape Pad Adhesive and c) HandiPrint Adhesive prior shooting. No interferences present for elements of interest from negative control Whatman paper, SIRCHIE Tape Pad, and HandiPrint Adhesive samples.

\subsubsection{Assessment of Shooting Substrate Selection}

Three substrates were chosen for this study as a representative group of materials commonly encountered, and tested for bullet holes, at firearm related crime scenes. Plywood, float glass, and drywall are all common surfaces in residential buildings, including doors, windows, and walls of a home. All substrates were purchased from a local hardware store to further ensure the materials in this study represented items easily obtained or purchased.

Each substrate was secured to custom shooting structure to reduced fracturing or breaking of the material when being shot. The plywood samples remained intact after being fired, with minimal splintering around the bullet hole. The drywall samples did not crumble or break after being shot but did result in some gypsum powder being deposited directly around the bullet hole. However, the bullet hole itself was a clean circle with no jagged edges. The float glass samples showed fracturing throughout the whole sample after being shot. The float glass purchased for this study is thinner than the architectural glass usually found in residential buildings. Therefore, packaging tape was used to keep the integrity of the glass after shooting, allowing for sampling of GSR to around the entrance orifice. Firearm discharge residues had high contrast against the white background of the drywall, resulting in an easy determination of the bullet hole overall physical characteristics. However, the texture of the plywood made it difficult to detect all the residues deposited on the substrate and were easily overlooked. Furthermore, the transparent nature of glass 
made it nearly impossible to see residues left behind by the firearm. The latter cases illustrate the difficulties associated with identifying bullet holes at crime scenes when sequences of events are not known. Figures 26 through 28 below show each substrate before and after being fired at.

Background spectra, using a single $5 \mathrm{~mm}$ ablation line by LIBS, were completed in triplicate for each substrate to determine any interfering elemental emission lines with those of interest ( $\mathrm{Sb}$ $(259.8 \mathrm{~nm}, \mathrm{I}), \mathrm{Pb}(405.8 \mathrm{~nm}, \mathrm{I})$, and $\mathrm{Ba}(493.4 \mathrm{~nm}, \mathrm{II}))$. No interferences were seen in any of the nine background spectra obtained (Figure 29).
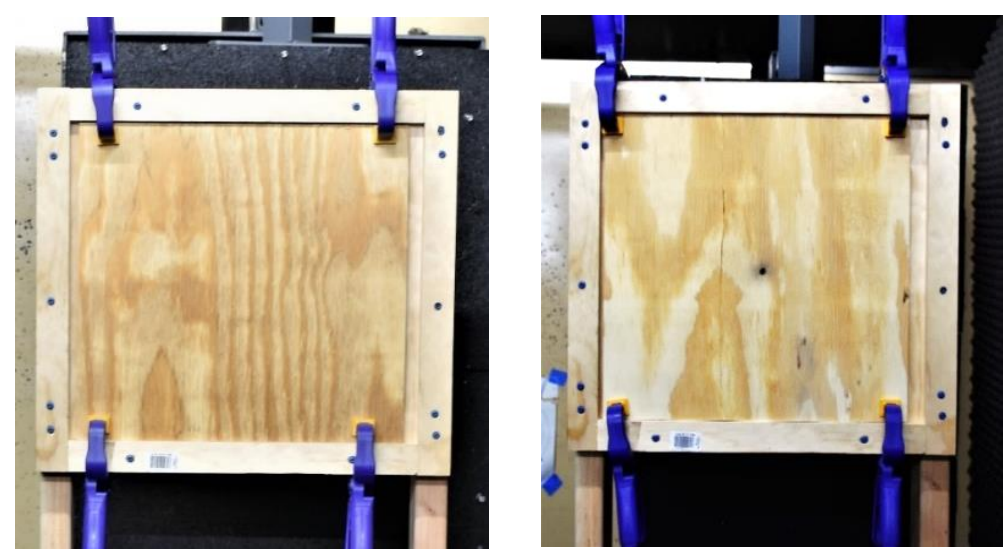

Figure 26. Plywood substrate secured to custom made shooting structure using clamps. Left: plywood before shooting. Right: Plywood with visible firearm discharge residues after being shot.
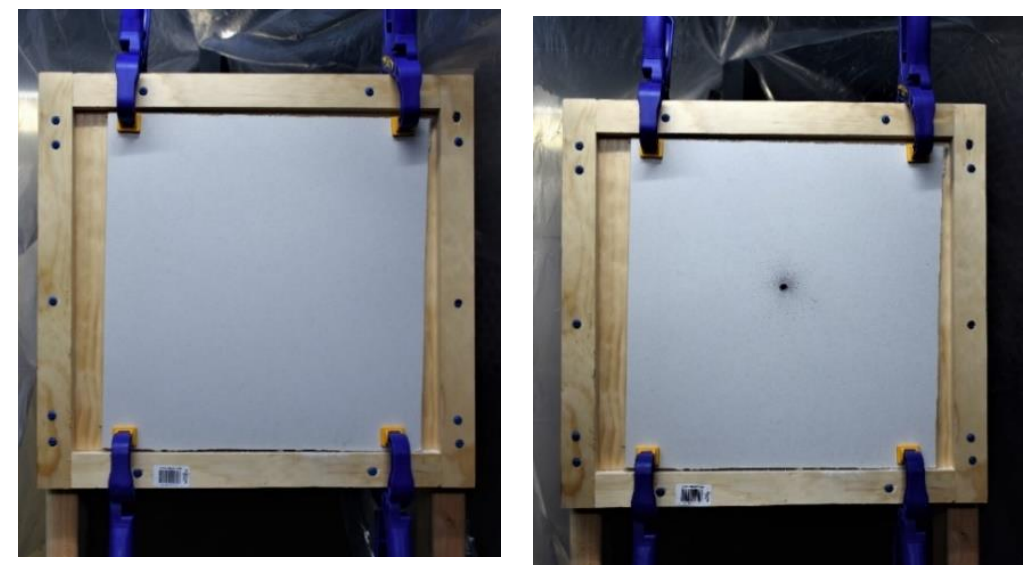

Figure 27. Drywall substrate secured to custom made shooting structure using clamps. Left: Drywall before shooting. Right: Drywall with visible firearm discharge residues after being shot. 

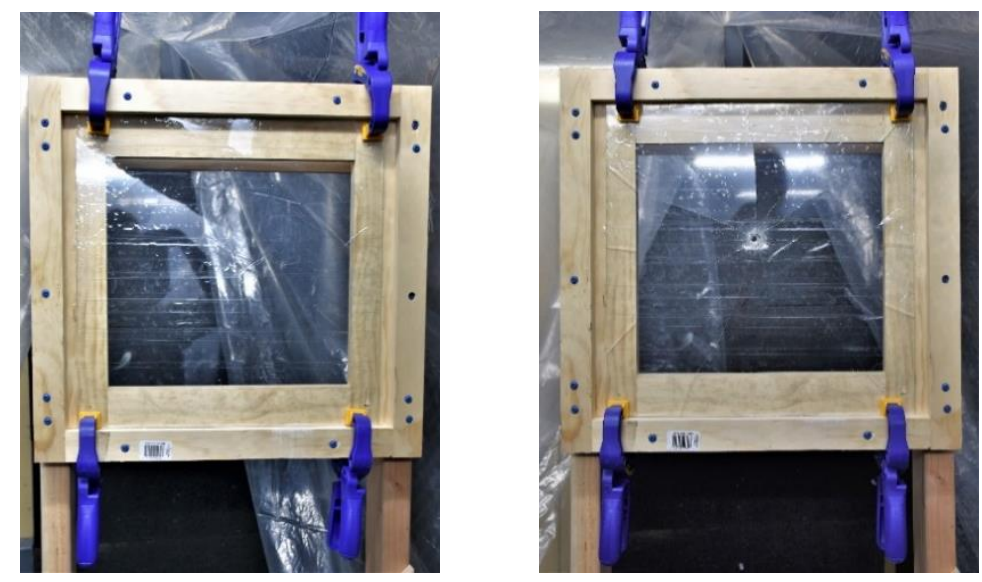

Figure 28. Glass substrate secured to custom made shooting structure using clamps. Left: Glass before shooting. Right: Glass with visible bullet hole after being shot.

a)

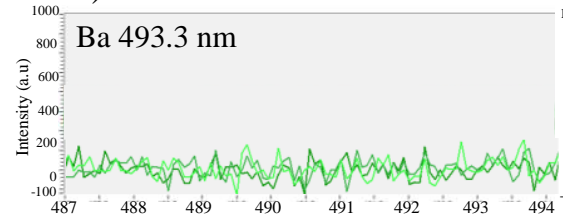

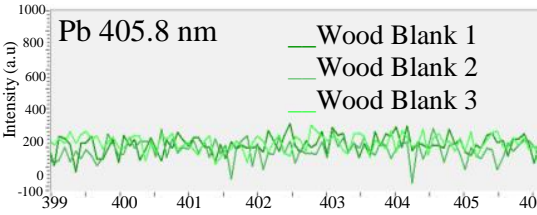

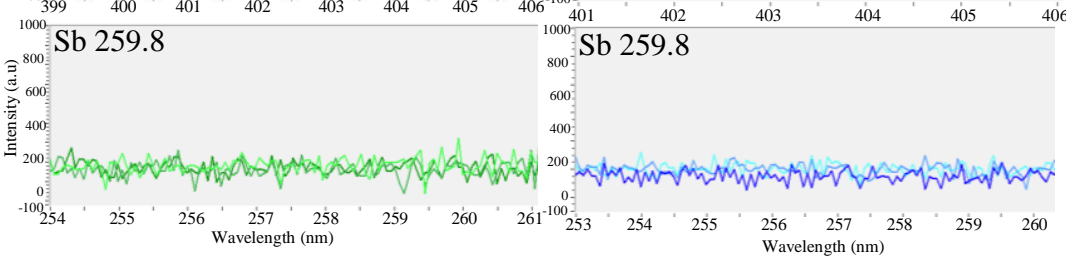

b)

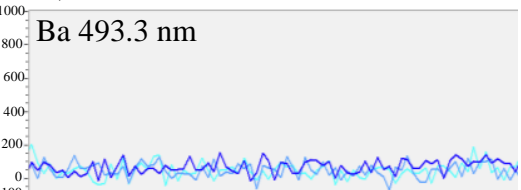

c)
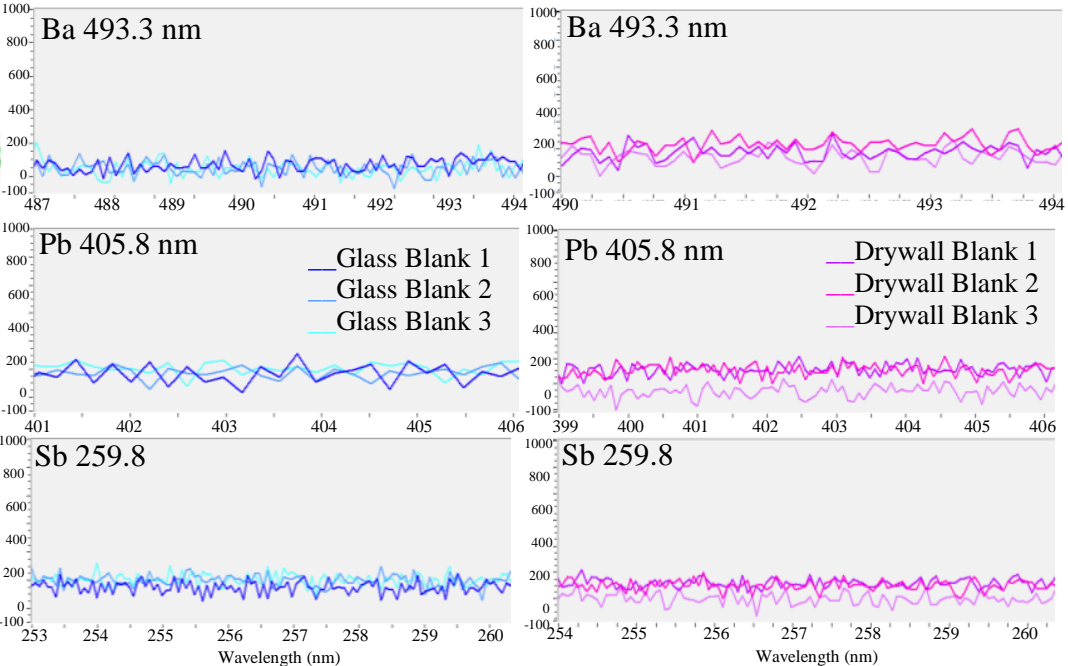

Figure 29. LIBS background spectra for a) wood b) glass and c) drywall prior shooting. No interferences present for elements of interest from negative control wood, glass, and drywall samples.

\subsubsection{Collection of Gunshot Residues from Wood, Drywall, and Glass Samples}

HandiPrint adhesive were applied to each substrate, centered around the bullet hole. A uniform pressure was applied to the entire area of the adhesive to ensure optimal transfer of gunshot residues. The Handiprint adhesive removed from each substrate with increasing difficulty; wood, drywall, glass. The rough texture of the plywood made it easy to remove the adhesive since the graining of the wood left voids where the adhesive was not sticking to the plywood. When removed, the wood itself left some residue on the adhesive. However, soot residues and gun 
powder particles were still visible, and the void left by the bullet hole was visible (Figure 30). The paper coating on the drywall posed a problem when removing the adhesive for LIBS analysis. The paper fixed itself to the adhesive in some areas, masking any gunshot residues that may be present. In some instances, whole sections of the adhesive were covered by the paper and in other instances only small areas were covered (Figure 31). Despite this issue, the location of the bullet hole was still discernable due to a darker grey/black coloring left on the white adhesive by the gunshot residues present. Removing the adhesive from the float glass samples was the most difficult. The smooth, hard surface of the glass resulted in a strong adhesion between the two substrates, requiring excessive pulling to remove the adhesive from the glass. The removal process caused the adhesive to stretch and become disfigured in areas (Figure 32). The disfigured areas were remedied once the adhesive was secured to the custom-made ablation stage. The way the bullet impacted the glass resulted in a larger area on the adhesive where the bullet hole was located compared to the plywood and drywall samples.

The concentric ablation pattern created as a rapid analysis to determine whether a hole in an object or substrate was created by a bullet. When a bullet comes into contact with an object, residues coming from the bullets itself, the primer, and propellant are spread on the target in a specific pattern and distribution. This concept suggests that if a bullet hole is present, these residues will be detected directly around the bullet, in an area close to the bullet hole, and will eventually become undetectable further away from the bullet hole. Therefore, a more objective, rapid, and non-destructive LIBS ablation pattern was developed to represent this notion. Three squares were used to detect residues at different areas on the sample. One directly around the hole, one $25 \mathrm{~mm}$ away from the bullet hole, and one that was in the corner, a significant distance from the bullet hole. A $25 \mathrm{~mm}$ distance between the first two squares was chosen based on the results from Chapter 1, showing that residues can be seen $100 \mathrm{~mm}$ away from the bullet hole when shot at from distances up to 36 inches. The proposed method gives a representative example of the GSR distribution that is present on a substrate that has been shot at. 


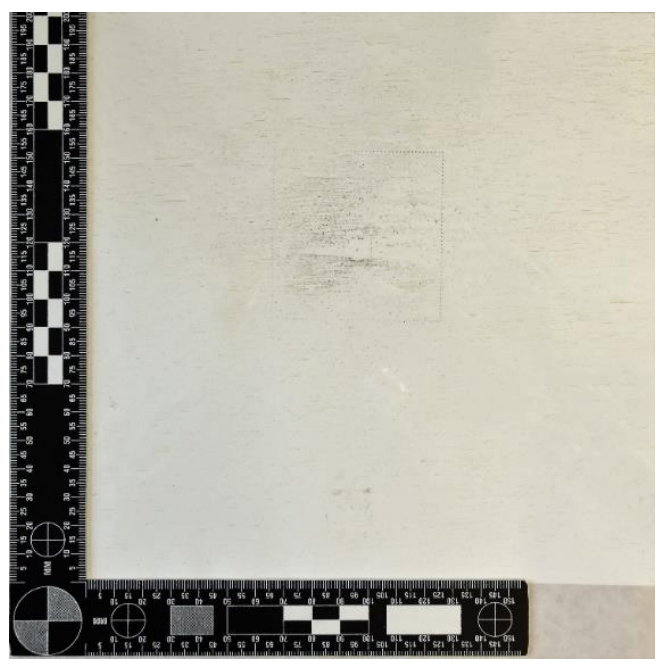

Figure 30. HandiPrint adhesive after transferring gunshot residues off of a wood substrate.
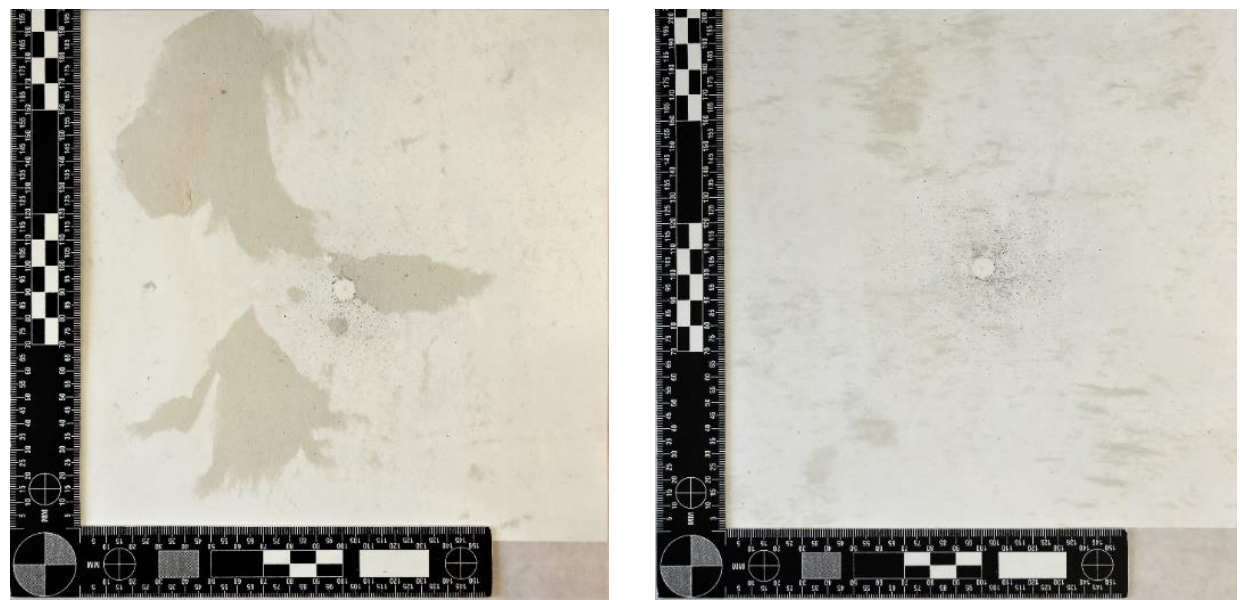

Figure 31. HandiPrint adhesives after transferring gunshot residues off of drywall substrates. Left: Large sections of adhesive covered in the paper coating of drywall. Right: Small areas of adhesive covered in paper coating of drywall.

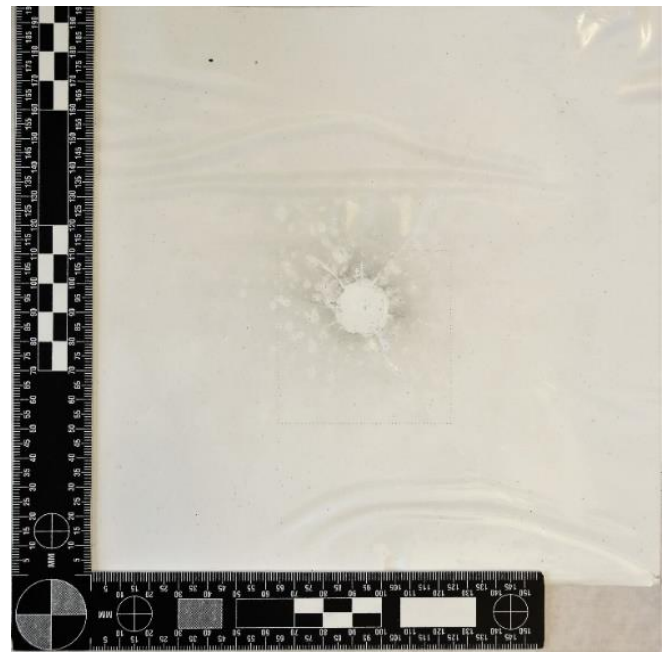

Figure 32. HandiPrint adhesive after transferring gunshot residues off of a glass substrate. 


\subsubsection{Assessment of LIBS Method and Chemical Maps for Bullet Hole Identification}

To visualize the spectral data obtained from the 21 samples, heat maps were created using JMP Pro 14 for each element of interest. The LIBS pattern performed created three squares consisting of multiple spots where a LIBS spectra was obtained. Overall, each map contains a total of 260 LIBS spectra. The negative sample of GSR, taken from the corner of the adhesive, was transposed to the center of the heat map to represent the blank area that is in the middle of a bullet hole. This was done to better visualize what a negative result for GSR would be compared to the positive results that would be see in the other two square ablation patterns on the samples. The result was three concentric squares, for every element of interest, for each adhesive sample. Examples of heat maps created from one wood sample, one drywall sample, and one glass sample can be seen below in Figure 33. The inherent low concentration of antimony in the Remington primer used in this study resulted in concentrations below detection limits when transferred to the adhesive. Therefore, heat maps were further created for only lead and barium.

Limitations mentioned above in Section 4.3.3., including wood sticking to the adhesive, the paper coating of the drywall masking residues, and the stretching of the adhesive when being removed from glass were assessed once the heat maps were created. The visible wood residues on the adhesive did not alter the heat maps, as the amount of wood background was negligible and was overcome by LIBS. Areas of the adhesive that were covered by the paper coating of the drywall were not able to detect residues present, and therefore, appeared as uncolored areas in the heat maps. The intermittent areas of no color are an additional indication that the drywall is not contributing to any lead or barium detected on the adhesive. Furthermore, the stretching seen on the adhesives removed from the glass did not alter or disrupt the distribution of gunshot residues transferred. However, the original $10 \mathrm{~mm} \times 10 \mathrm{~mm}$ square pattern had to be adjusted to accommodate for the larger bullet holes created in the glass samples. 


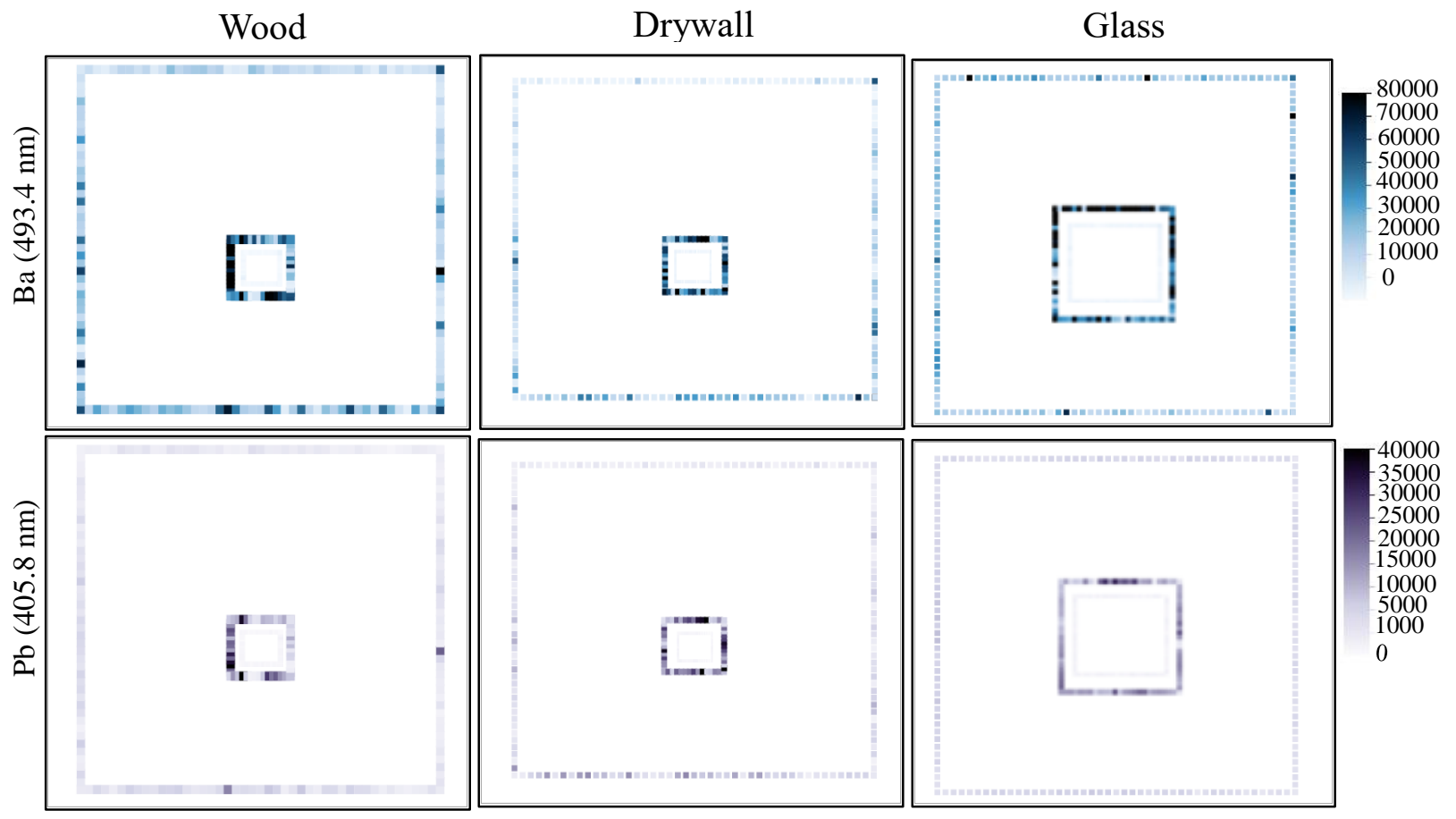

Figure 33. Heat maps of barium $(493.4 \mathrm{~nm})$ and lead $(405.8 \mathrm{~nm})$ for bullet hole identification, obtained set one of substrates. Left: Wood; Middle: Drywall; Right: Glass.

\subsubsection{Identification of Bullet Holes}

The overall goal of this objective was to determine if LIBS can be used as an analytical tool to identify suspected bullet holes in non-movable substrates found at a crime scene. Therefore, a sample was considered positive for bullet hole identification when lead and barium were detected in two of the three ablation squares; specifically, the patterns that were $10 \mathrm{~mm}$ x $10 \mathrm{~mm}$ in size and $60 \mathrm{~mm}$ x $60 \mathrm{~mm}$ in size around the bullet hole. A negative bullet hole identification, for this study, was when all three ablation patterns tested negative for gunshot residues.

All 21 samples in this study were considered positive for bullet hole identification under the preset conditions. For proof of concept, an additional textile sample was burned with a lit cigarette to simulate a situation where it is not obvious whether a suspected entrance hole was created by a bullet hole. Cigarettes burn holes in textiles leaving singed fibers and black residues, much like bullets leave when they come in contact with target materials. Residues were transferred from this textile sample using the HandiPrint adhesive and analyzed by LIBS using the same parameters as all other samples. The heat map created from the spectral data shows that neither lead and barium were detected anywhere on the sample (Figure 34). Therefore, this sample was considered a 
negative bullet hole identification. The results of this study show the importance of this rapid LIBS technique for bullet hole identification and GSR pattern visualization when recreating the events of crime scene. Moreover, the proposed method serves as proof of principle that can be extended to portable instrumentation for application "on site".
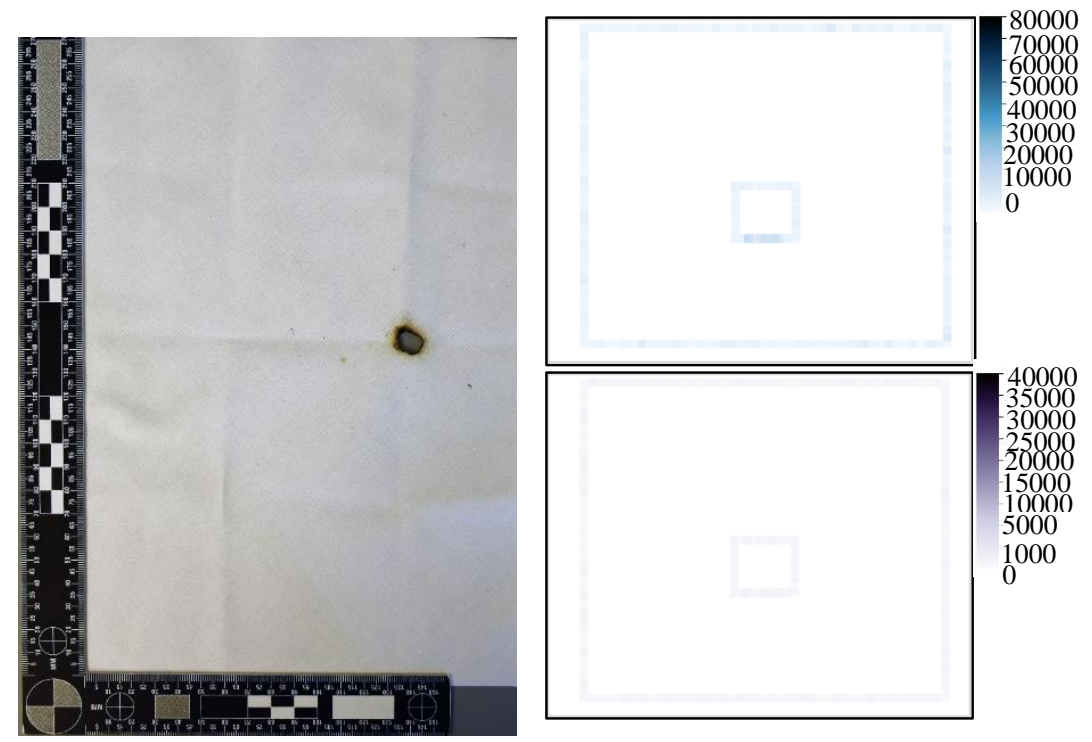

Figure 34. Left: Photograph of a white colored textile burned with a cigarette to simulate a bullet hole. Right: Heat maps created from the spectral data obtained from the sample for Top: Ba $(493.4 \mathrm{~nm})$, Bottom: $\mathrm{Pb}(405.8 \mathrm{~nm})$.

\subsection{Conclusions}

It is not always easily discernable whether a hole in a material was created by a bullet or another instrument. In these cases, it is important to employ methods that provide a rapid but accurate answer to the question at hand. Current nitrate and lead chemical detection methods used for bullet hole identification lack specificity for gunshot residues and are difficult to apply in-field when the hole is located on a large, non-movable surface at the crime scene. This study aimed to provide an advanced method for bullet hole identification by introducing a simple technique to transfer gunshot residues from these surfaces and utilizing LIBS to provide more reliable results. The results of this study show that transferring gunshot residues for analysis by LIBS is advantageous over conventional methods for bullet hole identification.

Transferring gunshot residues from the surface at the crime scene onto an adhesive allows the sample to be taken back to the laboratory before any analyses are performed. This lessens the chance of further contamination from the crime scene while making it simpler to perform the necessary analyses. The HandiPrint adhesive proved to be excellent at picking up gunshot residues 
from multiple surface that are commonly encountered at crime scene and are often subject to being shot at or are accidental hit by a ricocheting bullet.

Laser induced breakdown spectroscopy in this application requires no further sample preparation, is minimally destructive, provides more objective gunshot residue patterns, and increases the confidence of the presence of elements that are associated with gunshot residues.

The proposed method eliminates the use of chemical color tests traditionally used to detect nitrate or lead residues possibly present around a suspected bullet hole. These conventional methods require excessive chemicals that are hazardous and destroy important pattern evidence. Since the concentric LIBS pattern created during instrumental analysis is minimally destructive, the overall pattern of FDR on the adhesive remains intact, allowing for subsequent analyses to be performed if necessary. Additionally, with the custom ablation stage, no further preparation of the adhesive is required once it is collected off the substrate at the crime scene.

Additionally, LIBS has the capability of detecting multiple emission lines for element associated gunshot residues $(\mathrm{Pb}, \mathrm{Ba}, \mathrm{Sb})$, increasing the confidence of its presence in a sample. This is unlike color tests that illicit a color change from several materials other than gunshot residues and may lead to a false conclusion. Furthermore, these emission lines detected by LIBS can then be used to create accurate and objective chemical heat maps that represent the spatial distribution of gunshot residues on a sample or surface; helping to identify whether the hole in question was produced by a bullet.

This study is suspected to help provide crucial information when there is a need for reconstruction of events during crime scene investigations. The proposed method can help to identify whether a firearm was implemented in a crime when no weapon is located, or leading information is limited. 


\section{CHAPTER 3: THE EVALUATION OF THE USE OF LIBS FOR GSR DETECTION OF MULTIPLE PRIMERS AND BULLET TYPES}

\subsection{Project Overview}

This chapter proposes the application of Laser-Induced Breakdown Spectroscopy (LIBS) as a rapid technique for the identification of compounds present in standard and non-toxic ammunitions. Recent developments towards safer ammunition include bullets and primers that are non-toxic or "green" in nature. These advancements diminish the ability of chemical color tests to detect firearm discharge residues on target material, as they are insensitive to replacements for heavy metals. The ammunition chosen are a representative collection of possible ammunitions in market, including standard ammunitions, lead free ammunitions, and ammunitions that are heavy metal free. A total of 8 ammunitions (4 standards and 4 non-toxic) were used to shoot clothing samples from a close range. The samples were then analyzed by LIBS using a rapid spectral mapping method. A total of 11 elements, suggested by the ASTM E1588-17 ${ }^{9}$ for primer residues, were monitored during data acquisition. The presence or absence of inorganic elements surrounding the bullet hole were used to determine the composition of the ammunition and primer. Standard ammunitions consistently showed a presence of lead, barium, antimony, and other elements commonly associated with gunshot residues. Spectra obtained from non-toxic ammunitions showed difficulty for element detection and identification. While this is a start to detection of non-toxic ammunition, continued research using other instrumental techniques needs to be completed to enhance the findings of this study. Current efforts are being conducted in our research group to characterize these primers by ICP-MS and SEM-EDS analysis. Figure 35 shows a detailed layout of the number of samples, objectives, and specific analyses performed for each sample set. 


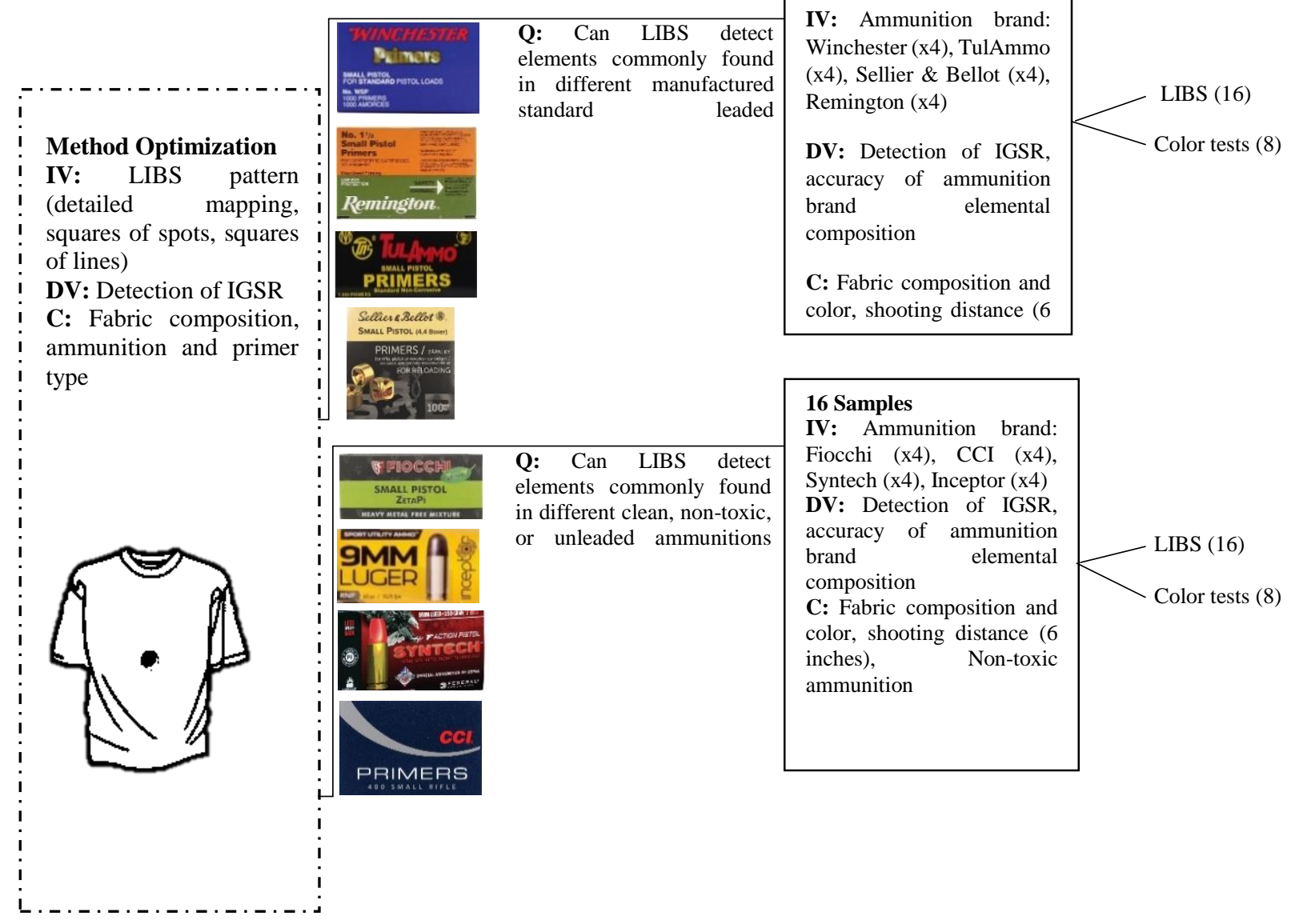

Figure 35. Experimental design for identification of ammunition composition by analysis of IGSR using LIBS including ammunition types/brands, variables, number of samples for each ammunition, and analyses performed for each sample. (IV= independent variable, $\mathrm{DV}=$ dependent variable, $\mathrm{C}=$ constant variable, $\mathrm{Q}=$ question)

\subsection{Materials and Methods}

\subsubsection{Firearms and Ammunitions}

A $9 \mathrm{~mm}$ Springfield XD9 pistol (manufactured in Croatia) and a Springfield XD .40SW pistol (manufactured in Croatia) were used for sample shooting and collection. The Springfield XD9 firearm was used for all leaded primers/ammunition and the Springfield XD .40SW firearm with an additional barrel was used for all non-toxic primers/ammunition. Eight different ammunitions were used in this study; 4 leaded ammunitions and 4 non-toxic ammunitions. The leaded ammunition consisted of Starline brass $9 \mathrm{~mm}$ Luger cartridge cases reloaded with Winchester 231 grain powder and Speer $9 \mathrm{~mm}$ Luger total metal jacket (TMJ) bullets. The reloaded leaded primers were manufactured by Remington, Winchester, Tulammo, and Sellier and Bellot. Two non-toxic ammunitions consisted of the same cartridge set up previously mentioned, with primers 
manufactured by CCI and Fiocchi. The two additional non-toxic setups were factory made ammunitions manufactured by Syntech and Inceptor. The setup manufactured by Syntech consisted of total synthetic polymer coated lead bullets, brass cartridge cases, and advertised lead free primers. Inceptor cartridges consisted of brass cartridge cases, copper-polymer matrix bullets, and advertised lead-free primers. The type of propellant used was unknown for both factory-made ammunitions. Total metal jacketed bullets were chosen for reloaded ammunition to ensure the majority of lead seen in LIBS spectra originated from the primer as opposed to the bullet itself.

\subsubsection{Sample Preparation and Collection}

Please refer to section $\underline{3.3 .1 .1}$. for sample preparation and shooting conditions.

\subsubsection{Experimental Setup}

The experimental layout was designed to aid in the identification of different types of ammunitions currently in the market. Manufacturers are currently developing ammunition that claim to be safer for individuals, as well as the environment. These new ammunitions have less concentrations, or may eliminate, components that contain heavy metals like lead and barium. The proposed LIBS method was designed to attempt to detect the changing composition of ammunitions currently available. The developed method is a rapid analysis that takes less than 30 minutes per sample and keeps the overall integrity of FDR intact. Negligible destruction of the sample by LIBS allows for subsequent analysis to be performed on the sample, including shooting distance determination using LIBS or conventional color tests if required. Additionally, other analytical techniques could be performed as confirmatory methods, including: laser-ablation inductively coupled plasma mass-spectrometry and scanning electron microscopy-energy dispersive X-ray spectrometry.

\subsubsection{LIBS Instrumentation, Parameters, and Methods}

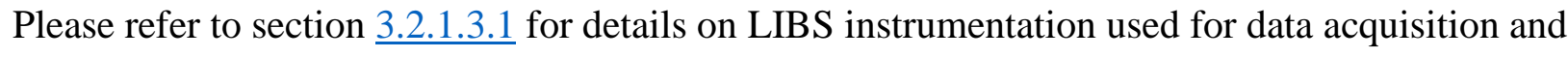
section $\underline{4.2 .4}$. for ablation patterns and parameters used for IGSR detection.

\subsubsection{LIBS Data Processing and Analysis}

Aurora software (Applied Spectra; Freemont, CA) was used for data pre-processing, including background subtraction, peak identification, and peak area integration. The selected elemental peaks of interest were chosen to encompass products found in both leaded and non-toxic ammunitions. The following wavelengths were monitored during analysis: $\mathrm{Sb}(259.8 \mathrm{~nm}, \mathrm{I}), \mathrm{Pb}$ 
(405.8 nm, I), Ba (493.4 nm, II), Al (396.1 nm, I) Ti (334.9 nm, I), Zn (481.0 nm, I), Ga (417.2 nm, I), Cu (324.7 nm, I), Sn (326.2 nm, I), Gd (310.0 nm, I), Sr (407.7 nm, II), Zr (343.8 nm, II), $\mathrm{Ni}(361.9 \mathrm{~nm}, \mathrm{I}), \mathrm{P}(213.6 \mathrm{~nm}, \mathrm{I})$, and S $(545.4 \mathrm{~nm}, \mathrm{II})$. However, one advantage of LIBS is the ability to collect simultaneous elemental information from $200 \mathrm{~nm}-1000 \mathrm{~nm}$ without need of preselected elements. As a result, the spectra can be examined after acquisition to identify other potential FDR components. Additional emission lines used for confirmation of presence can be seen below in Table 12. The integrated spectral data was preprocessed using Excel 2016 (version 15.24, Microsoft Corporation). Chemical maps and multivariate statistical methods were performed on JMP Pro (version 14.0.0; SAS Institute Inc., NC).

Table 12

Wavelengths Monitored for Elements of Interest in Leaded and Non-Toxic Ammunitions

\begin{tabular}{|c|c|c|c|}
\hline Element & Wavelength (nm) & Element & Wavelength (nm) \\
\hline Gadolinium & $\begin{array}{l}310.0, \text { II } \\
336.2 \text {, II } \\
303.3, \text { II }\end{array}$ & Strontium & $\begin{array}{l}\text { 407.7, II } \\
430.5, \text { II } \\
421.6, \text { II }\end{array}$ \\
\hline Tin & $\begin{array}{l}326.2, \mathrm{I} \\
284.0, \mathrm{I} \\
317.5, \mathrm{I}\end{array}$ & Zirconium & $\begin{array}{l}343.8, \text { II } \\
339.2 \text {, II } \\
349.6, \text { II }\end{array}$ \\
\hline Zirconium & $\begin{array}{l}481.0, \mathrm{I} \\
334.5, \mathrm{I} \\
472.2, \mathrm{I}\end{array}$ & Nickel & $\begin{array}{l}361.9, \mathrm{I} \\
352.5, \mathrm{I} \\
341.5, \mathrm{I}\end{array}$ \\
\hline Gallium & $\begin{array}{l}417.2, \mathrm{I} \\
403.3, \mathrm{I} \\
294.4, \mathrm{I}\end{array}$ & Phosphorous & $\begin{array}{l}213.6, \mathrm{I} \\
253.6, \mathrm{I} \\
255.3, \mathrm{I}\end{array}$ \\
\hline Copper & $\begin{array}{l}324.7, \mathrm{I} \\
327.4, \mathrm{I} \\
521.8, \mathrm{I}\end{array}$ & Sulfur & $\begin{array}{l}545.4, \text { II } \\
543.3 \text {, II } \\
563.9, \text { II }\end{array}$ \\
\hline Titanium & $\begin{array}{l}334.9, \text { II } \\
376.1 \text {, II } \\
368.5 \text {, II }\end{array}$ & & \\
\hline
\end{tabular}




\subsubsection{Physical Examinations and Color Tests}

Initial examination of all samples began with physical characteristics of the discharge residues originating from each of the 8 different ammunitions used in this study. Macroscopic characteristics, including soot appearance and color, were recorded first followed by microscopic characteristics of the gunpowder particles using a Leica light microscope.

Please refer to section 3.2.1.3.3. and for color tests methods and reagents used for analysis. Color tests were performed on two samples for each ammunition and the other two samples were subjected to other confirmatory methods including SEM-EDS.

\subsection{Results and Discussion}

In recent years, the ammunition market has evolved with development of non-toxic ammunitions that eliminate the use of some or all heavy metals used in traditional ammunitions for ignition of the primer and propellant. These changes limit the use of traditional color tests that are selective for those heavy metals, including lead and barium. Therefore, the need for an advanced detection method is necessary to aid forensic investigators in identifying and analyzing these progressive ammunitions. This study aimed to provide an analytical technique to detect and identify the replacement components in multiple non-toxic ammunitions.

\subsubsection{Visual Characteristics of Firearm Discharge Residues}

Visual characteristics were initially examined to attempt to differentiate the ammunitions by physical appearance of the firearm discharge residues. The standard reloaded ammunitions (Winchester, Remington, Sellier and Bellot, and TulAmmo) all had similar appearances. This was expected due to all standard ammunitions being reloaded with the same bullet, propellant, and cartridge cases. Concentric soot circles, due to the close-range shooting distance of 6 inches, were apparent on all samples fired at with a standard ammunition. The inner ring appeared darker in color compared to the outer circle of soot residues. During macroscopic examination, the gunpowder particles appeared to be partially burnt flakes that were dark grey or black in color. However, under microscopic examination, the particles were, in fact, dark green and light green colored discs. The soot surrounding the particles made the particles appear darker in color when looking at the overall FDR pattern of the samples. The visual similarities made it impossible to 
differentiate between standard ammunitions when considering only macroscopic and microscopic characteristics (Figure 37).

In contrast, the textiles shot with non-toxic ammunitions were easily separated from those shot with standard ammunitions (Figure 38). The initial observation was the appearance of the soot residues produced by the non-toxic ammunition. No soot was detected on the textiles shot with the reloaded CCI and Fiocchi ammunitions, while the factory-made Inceptor ammunition produced light grey soot residues that were concentrated around the bullet hole. On the other hand, textiles shot with the Syntech ammunition had black soot residues that were visibly similar to the patterns created by the standard ammunitions.

The gunpowder particles present on textiles shot by cartridges reloaded with the CCI and Fiocchi primers were initially recorded as dark green. Upon microscopic examination both light green and dark green particles were observed, most of which were partially burnt with minimal unburnt particles. Gunpowder particles observed for these two ammunitions were similar to those observed for the standard ammunitions due to identical reloading processes. Gunpowder particles observed on textile samples shot with Inceptor ammunition initially appeared to be tan in color but were confirmed to be yellow and light green when overserved under the microscope. Similarly, the gunpowder particles produced by the Syntech ammunition were yellow and black in color under the microscope. Unlike all other gunpowder particles observed in this study, the majority of the particles observed for the two factory made ammunitions (Inceptor and Syntech) were unburnt particles in their original morphology. Furthermore, these samples lacked the melting, or singing, of the fibers that are characteristic of a bullet hole. The lack of burning evidence on these samples suggests that the manufactured non-toxic cartridge set-ups do not reach the same high temperatures as standard ammunitions upon discharge of the firearm. Additionally, the red polymer coating encasing the bullet, manufactured by Syntech, was observed under magnification as molten globules surrounding the bullet hole; adding another level of discrimination from other ammunitions. Photographs of gunpowder particles for each type can be seen in Figure 36. 
a)

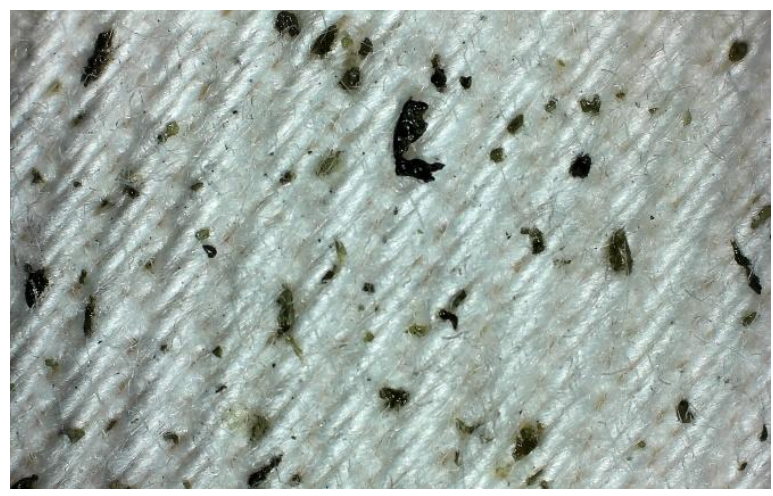

c)

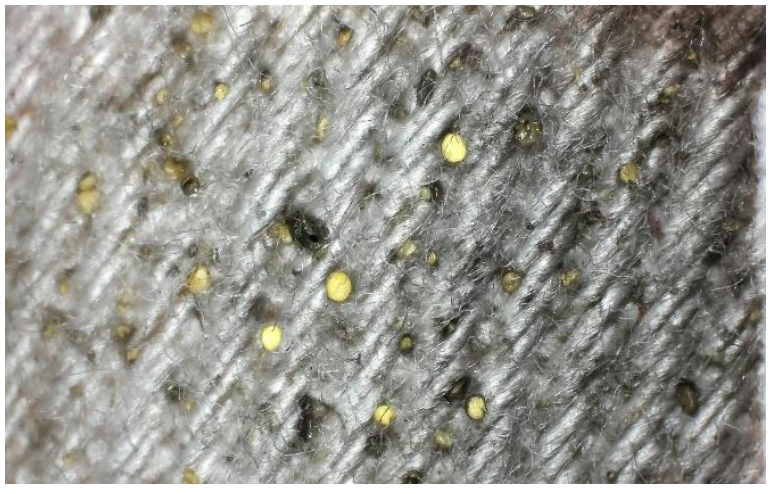

b)

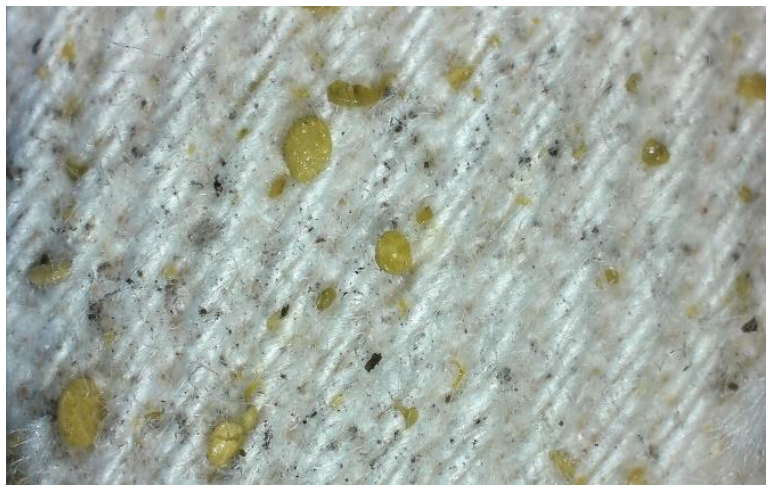

d)

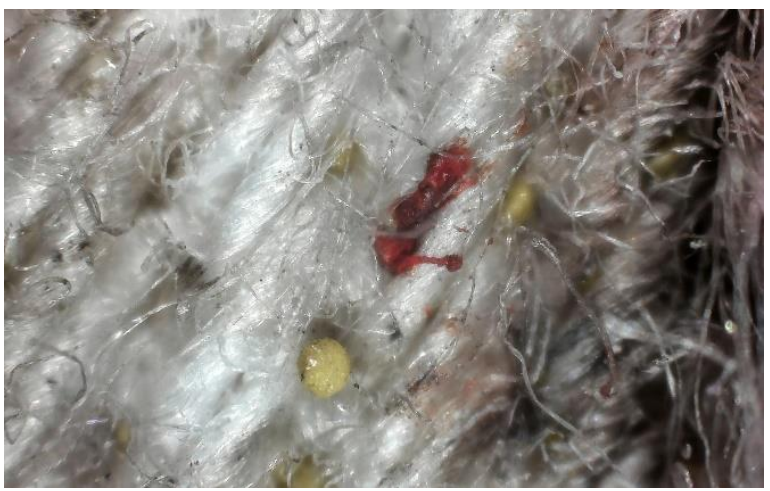

Figure 36. Microscopic photographs of gunpowder particles originating from the different standard and non-toxic ammunitions present in this study; a) Reloaded gunpowder in ammunitions using CCI, Fiocchi, TulAmmo, Remington, Winchester, and Sellier \&Bellot primers, b) gunpowder from factory-made Inceptor ammunition, c) gunpowder from factory-made Syntech ammunition, d) red polymer coating on bullet from Syntech ammunition.

\subsubsection{Chemical Colors Tests}

Sixteen samples, 2 from each ammunition, were subjected to the modified Griess test to observe colors changes indicative of nitrites being present. Positive control tests were performed on all four corners of the photographic paper prior to analysis of a sample. Measurements were not taken of Griess test results, as the presence or absence of color was of only importance.

Textiles shot with standard ammunitions (Winchester, Remington, Sellier and Bellot, and TulAmmo) produced similar patterns of orange color due to a consistent close-range shooting distance. The darkest orange color was concentrated around the bullet hole and faded as the distance from the bullet hole increased. Additionally, a darker orange color was observed on the photographic paper where gunpowder particles were located on the sample (Figure 37). The 
overall appearance of each modified Griess Test result from these standard ammunitions is comparable to a standard clothing sample fired from a distance between 6 inches and 12 inches, as seen in Figure 15.
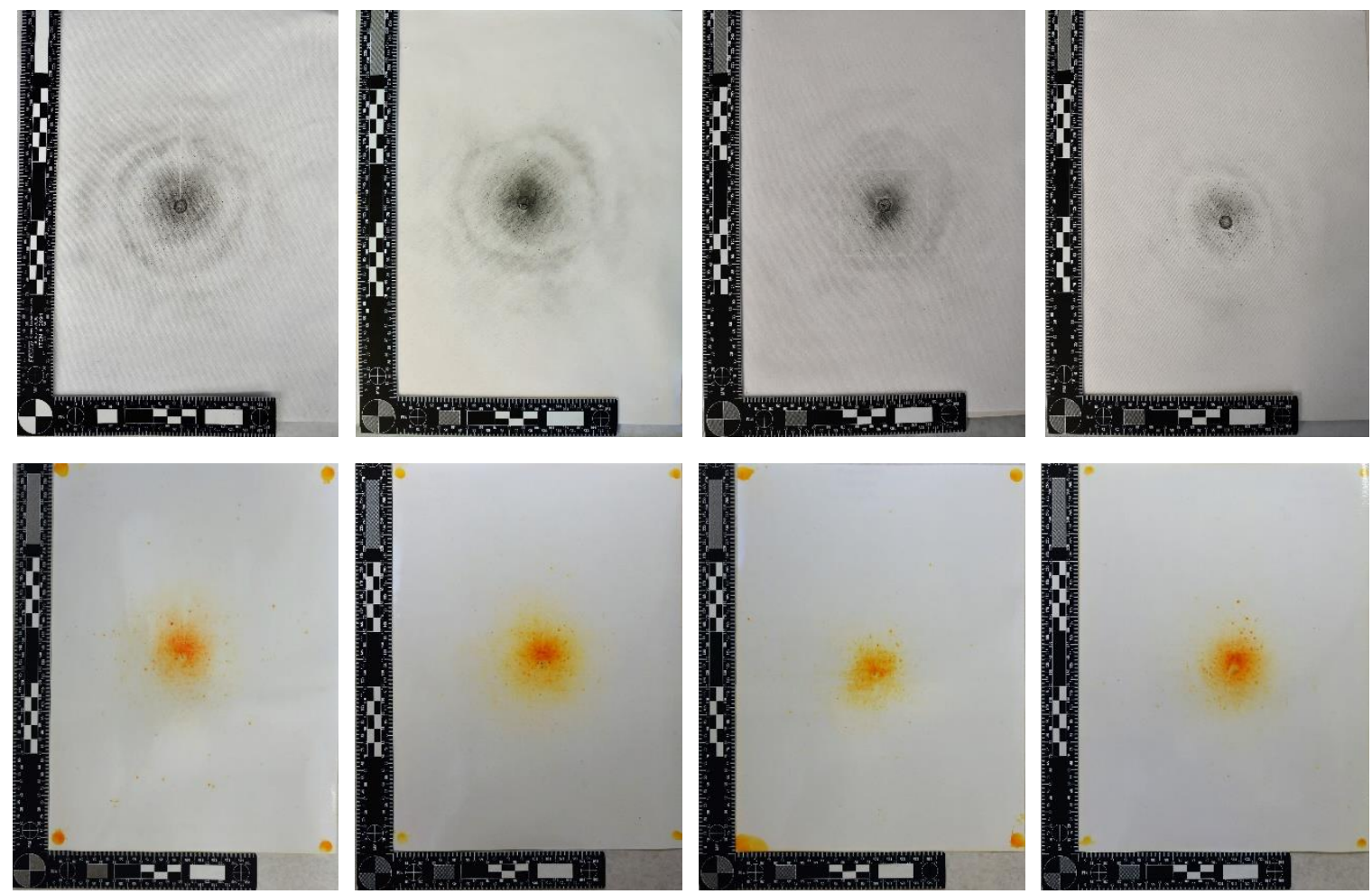

Figure 37. Photographs of original samples and results after the modified Griess test was performed on photographic paper (scale: $280 \mathrm{~mm} \times 150 \mathrm{~mm}$ ). One set of samples shot with standard ammunitions (from left to right: Remington, TulAmmo, Winchester, Sellier and Bellot)

modified Griess test results for non-toxic ammunitions (Fiocchi, Inceptor, Syntech, and CCI) provided little information for pattern recognition. An intense orange color developed on the photographic paper anywhere the textile and cheese cloth came in contact (Figure 38), resulting in large orange squares that were uniform in color. Areas not exposed to the cheese cloth remained white. An unfired textile was tested using the same process to assess contamination of reagents or materials used in testing. No orange color developed for this sample, apart from the positive control tests done on the four corners of the photographic paper. The uniform orange color suggests an immense amount of organic materials present in non-toxic ammunitions compared to standard ammunitions. It is hypothesized that these organic materials are mainly originating for the nontoxic primers, as the propellant used in two of the non-toxic ammunitions (Fiocchi and CCI) was also used in all standard ammunitions. Additionally, no purple color was detected when performing 
the sodium rhodizonate test on the samples. These results illustrate the limitations associated with the development of modern ammunitions and why further research is required to develop an enhanced detection method.
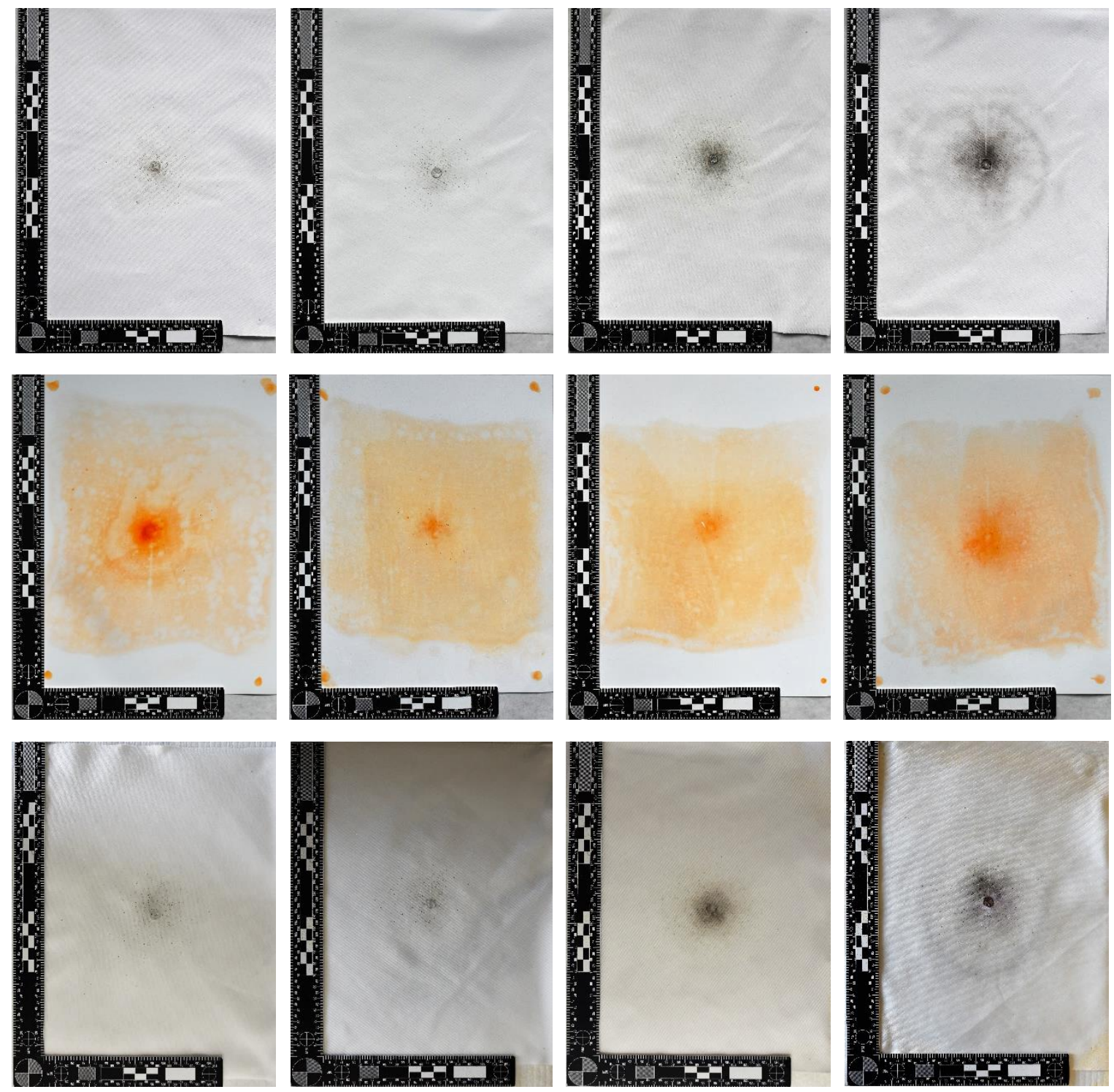

Figure 38. Photographs of original samples and results after the middle: modified Griess test and bottom: sodium rhodizonate test was performed on photographic paper (scale: $280 \mathrm{~mm}$ x $150 \mathrm{~mm}$ ). One set of samples shot with non-toxic ammunition (from left to right: Fiocchi, CCI, Inceptor, Syntech)

\subsubsection{Identification of Elements Using Laser Induced Breakdown Spectroscopy}

Initial processing of the spectral data included integrations of a signal and noise for each element of interest. The noise integration was performed on a level background area of the spectrum close to the signal of interest. The integrated spectral data for each sample was further processed to calculate the signal to noise ratio for each emission line of interest. An element was considered 
present in the spectrum when the signal to noise ratio reached a set threshold of 3 . A signal to noise ratio below this threshold indicated that element was not present.

Three background spectra were taken of the white fabric to observe any interferences with the chosen elements of interest. Interferences were only observed at $422.7 \mathrm{~nm}, 766.5 \mathrm{~nm}$, and 769.9 $\mathrm{nm}$, which are two characteristic emission lines for calcium and potassium, respectively. After further examination, peaks were seen at numerous emission lines corresponding to both calcium and potassium, confirming their presence in the textile. Signal to noise ratios were then modified these elements when determining presence or absence in a sample; average intensities taken from the three negative control samples were used as the noise for these elements as opposed to the "signal" noise previously described for other elements of interest.

Table 13 below shows the elements detected by LIBS in each ammunition that had signal to noise ratios greater than three.

Table 13.

Elements Detected in Standard and Non-Toxic Ammunitions by Laser Induced Breakdown Spectroscopy

\begin{tabular}{cl}
\hline Ammunition & Elemental Composition \\
\hline Leaded & \\
Remington & $\mathrm{Pb}, \mathrm{Ba}, \mathrm{Sb}, \mathrm{Cu}$ \\
TulAmmo & $\mathrm{Pb}, \mathrm{Sb}, \mathrm{Cu}$ \\
Winchester & $\mathrm{Pb}, \mathrm{Ba}, \mathrm{Sb}, \mathrm{Cu}$ \\
Sellier and Bellot & $\mathrm{Pb}, \mathrm{Ba}, \mathrm{Sb}, \mathrm{Cu}$ \\
Non-Toxic & \\
Fiocchi & $\mathrm{K}$ \\
Syntech & $\mathrm{K}, \mathrm{Ba}$ \\
Inceptor & $\mathrm{K}$ \\
CCI & $\mathrm{K}, \mathrm{Ba}$
\end{tabular}

It is anticipated that the potassium seen in all non-toxic ammunition is originating from an explosive component in the primer. It has been reported that potassium nitrate has been used in "clean" ammunition to replace the barium nitrate initiator in standard ammunitions ${ }^{72,73}$.

\subsubsection{Additional Testing Methods for Confirmation of Chemical Profiles}

Preliminary investigations of the standard and non-toxic ammunitions using scanning electron microscopy-energy dispersive X-ray spectroscopy and inductively coupled plasma-mass 
spectrometry were performed in an attempt to confirm elemental compositions of the gunshot residues. The morphology of the particles seen varied between primers; Fiocchi: spherical, Syntech: spherical and irregular, CCI: irregular and jagged edges, Inceptor: spherical and globular. All standard ammunitions produced particles typical of gunshot residues; spherical and $\sim 1 \mu \mathrm{m}$. Table 14 and 15 show the elements identified by SEM-EDS and ICP-MS for each standard and non-toxic primer. Figures 39-42 show examples of particles and SEM-EDS spectra for the various non-toxic ammunitions.

Table 14.

Elements Detected in Non-Toxic Ammunitions by SEM-EDS and ICP-MS

\begin{tabular}{cll}
\hline Ammunition & Signature Elements & Variable Elements \\
\hline SEM/EDS & & \\
Fiocchi & $\mathrm{Al}, \mathrm{Si}, \mathrm{K}$ & $\mathrm{S}, \mathrm{Na}, \mathrm{Ca}, \mathrm{Cu}, \mathrm{Fe}$ \\
Syntech & $\mathrm{Bi}$ & $\mathrm{Na}, \mathrm{K}, \mathrm{Al}, \mathrm{Cu}$ \\
Inceptor & $\mathrm{Ti}, \mathrm{Zn}$ & $\mathrm{S}, \mathrm{Na}, \mathrm{Ca}, \mathrm{K}$ \\
CCI & $\mathrm{Ba}$ & $\mathrm{S}, \mathrm{Na}, \mathrm{Si}, \mathrm{Ca}, \mathrm{Cu}, \mathrm{Al}$ \\
ICP/MS *primer only* & & \\
Fiocchi & $\mathrm{K}(7.9 \pm 0.1 \mathrm{ppm})$ &
\end{tabular}

Table 15.

Elements Detected in Standard Ammunitions by SEM-EDS and ICP-MS

\begin{tabular}{lll}
\hline Ammunition & Signature Elements & Variable Elements \\
\hline SEM/EDS & & \\
Remington & $\mathrm{Ba}, \mathrm{Sb}$ & $\mathrm{Pb}, \mathrm{Cu}$ \\
TulAmmo & $\mathrm{Pb}, \mathrm{Sb}$ & $\mathrm{Cu}$ \\
Sellier and Bellot & $\mathrm{Pb}, \mathrm{Ba}, \mathrm{Sb}$ & $\mathrm{Cu}$ \\
ICP/MS *primer only* & $\mathrm{Pb}(11.5 \pm 0.3 \mathrm{ppm}), \mathrm{Ba}(19.1 \pm 1.0$ & \\
Remington & $\mathrm{ppm}), \mathrm{Sb}(5.0 \pm 0.3 \mathrm{ppm})$ & \\
& $\mathrm{Pb}(56.5 \pm 1.2 \mathrm{ppm}), \mathrm{Sb}(7.1 \pm 0.2 \mathrm{ppm})$ & \\
TulAmmo & $\mathrm{Pb}(6.3 \pm 0.4 \mathrm{ppm}), \mathrm{Ba}(7.8 \pm 0.8 \mathrm{ppm})$, & \\
Sellier and Bellot &
\end{tabular}


For SEM-EDS, gunshot residues were collected directly off of fabric samples using a standard SEM stub. The GSR collected from textiles, using this procedure, encompassed an area about 3 times larger than the area of sample analyzed by the rapid LIBS mapping method. This extended sampling region concentrates the gunshot residues to a smaller area and collects a more representative sample of residues for each non-toxic or standard ammunition. Therefore, the expanded elemental profiles identified by SEM-EDS may be due to the more comprehensive GSR sampling method.
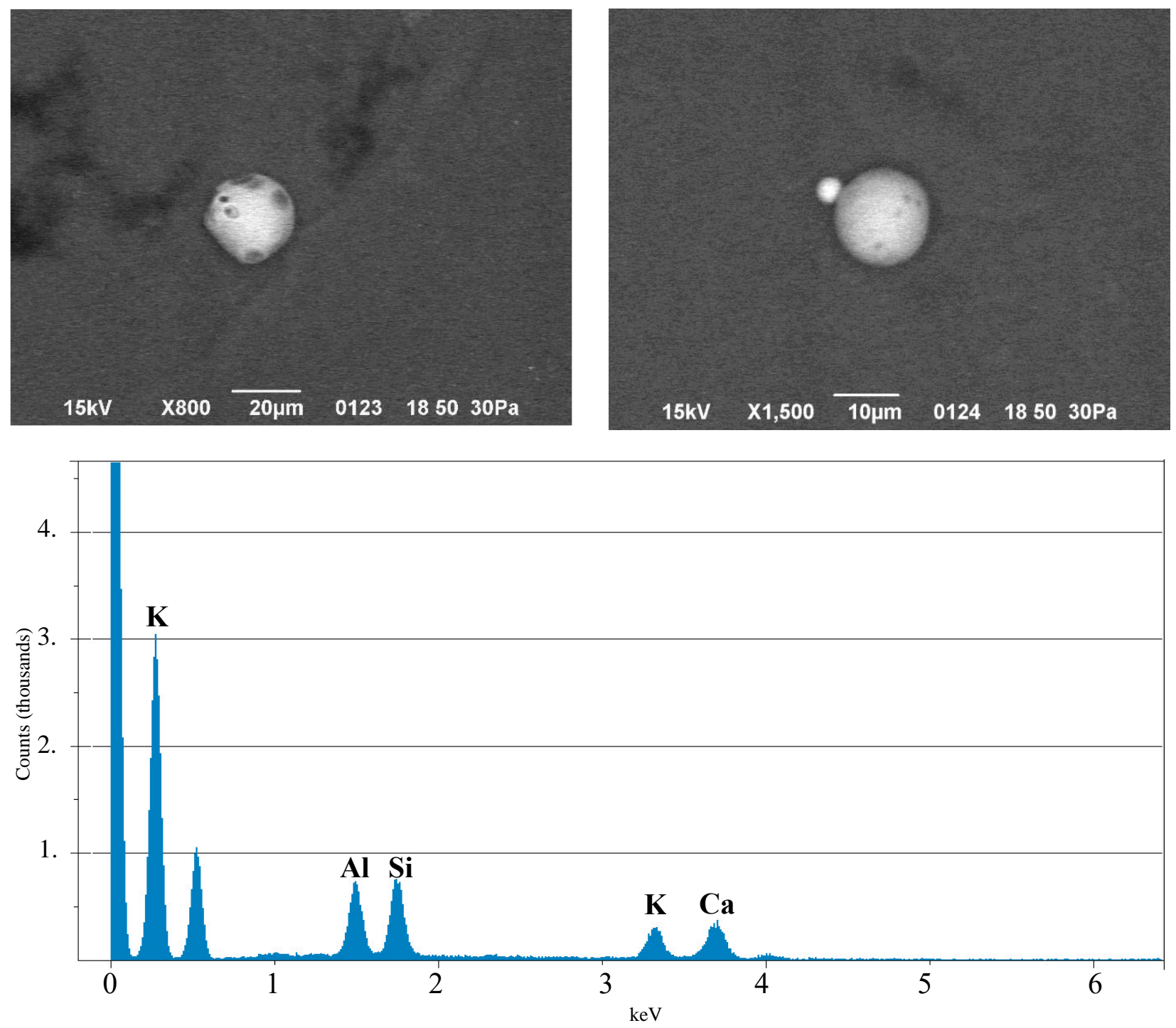

Figure 39. SEM-EDS images of the morphological characteristics of a GSR particle originating from a Fiocchi small pistol primer and its corresponding elemental profile. 

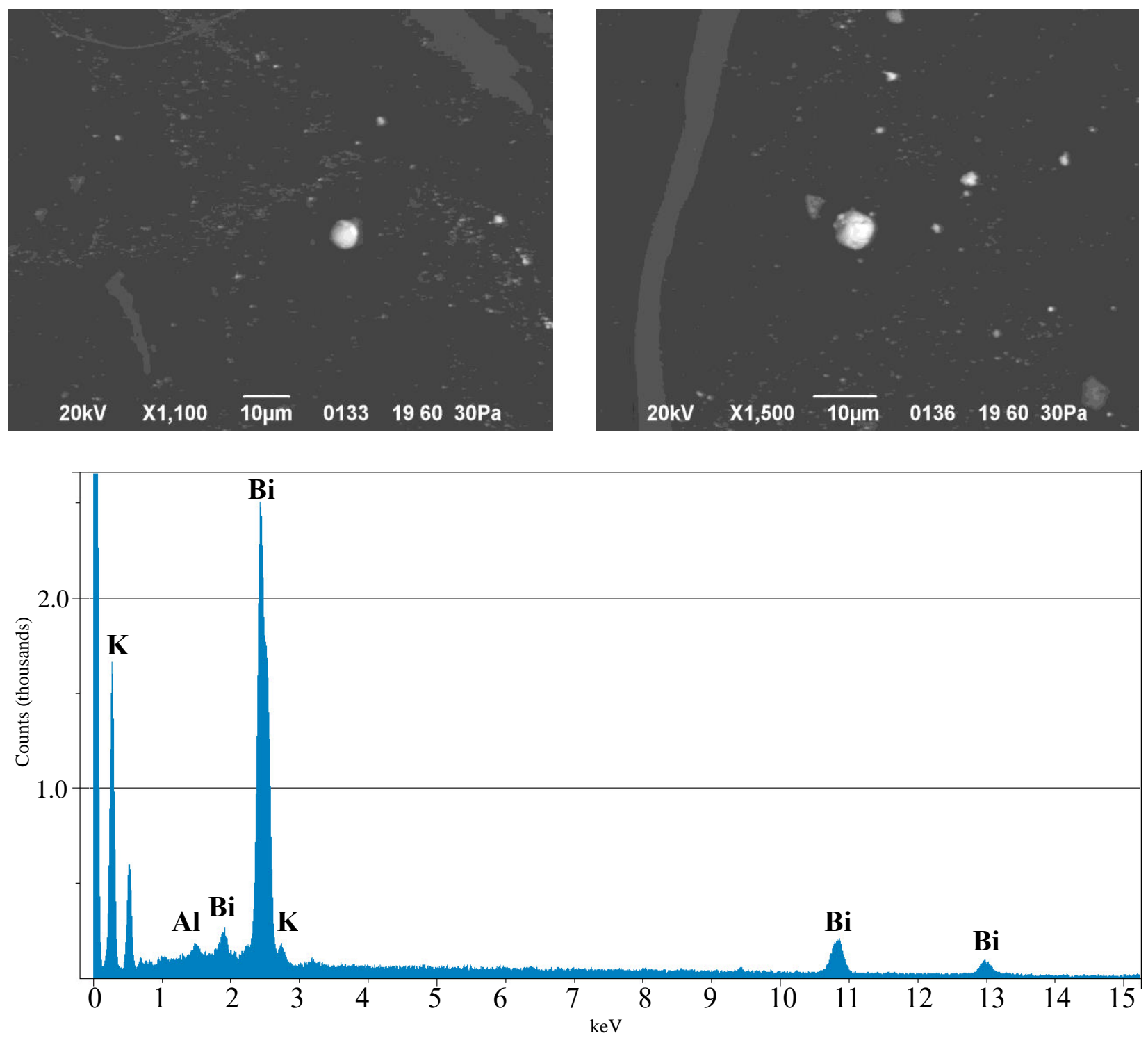

Figure 40. SEM-EDS images of the morphological characteristics of a GSR particle originating from a Syntech small pistol primer and its corresponding elemental profile. 

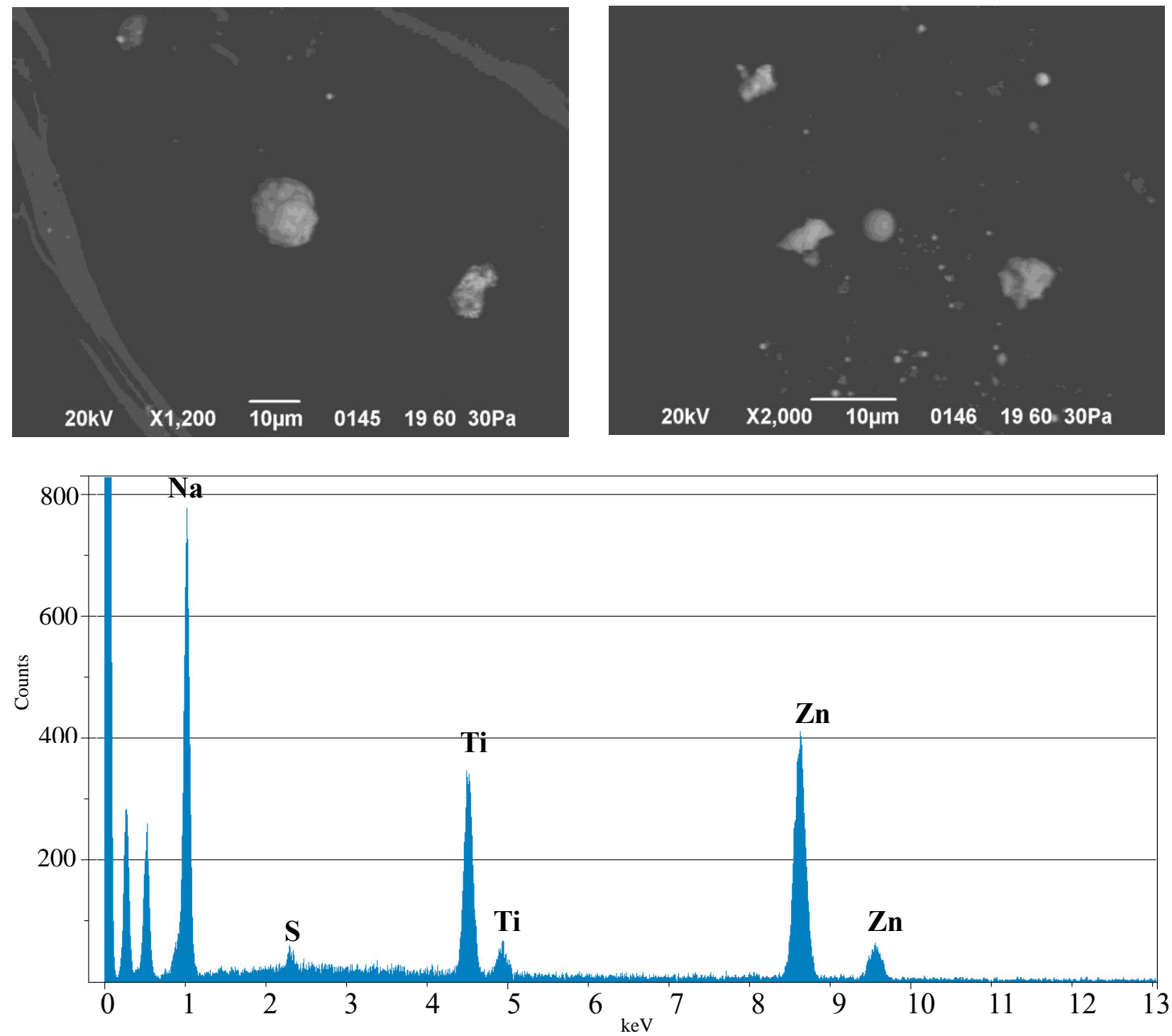

Figure 41. SEM-EDS images of the morphological characteristics of a GSR particle originating from an Inceptor small pistol primer and its corresponding elemental profile. 

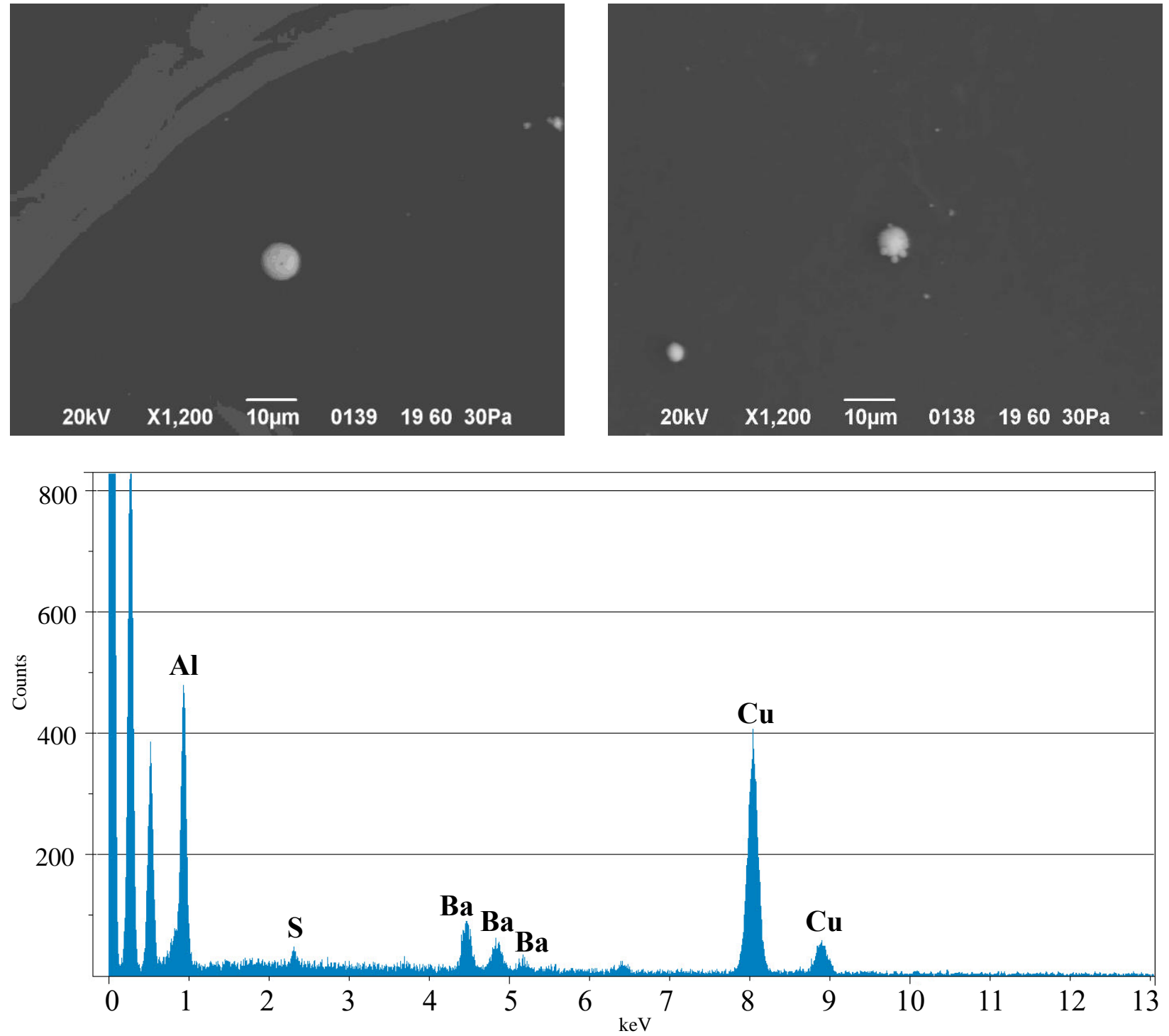

Figure 42. SEM-EDS image of the morphological characteristics of a GSR particle originating from a CCI small pistol primer and its corresponding elemental profile.

The SEM stubs, used to collect gunshot residues from the clothing samples, were re-analyzed by LIBS using a 5 x 5 grid of spots ( 25 total spots). Specific LIBS parameters used for the 25 spots can be seen in Table 16. Once again, an element was considered present in the sample if a single to noise ratio of at least 3 was reached. The SEM stubs allowed for a more concentrated amount 
of particles in a smaller area, as opposed to the clothing where only small sections of the clothing were ablated and the laser may not have interacted with a large number of particles. Therefore, a more extensive lists of elements were detected by LIBS on the stubs for each of the non-toxic ammunitions. The comprehensive list of elements detected by LIBS on each stub can be seen in Table 17.

Table 16 LIBS Parameters of Spot Pattern for Non-toxic Ammunition Detection

\begin{tabular}{ll}
\hline Parameter & Measure \\
\hline Ablation Pattern & 5 spot x 5 spot square \\
Spot Size & $100 \mathrm{um}$ \\
Number of shots per spot & 2 \\
Pulse repetition rate & $5 \mathrm{~Hz}$ \\
Pulse Energy & $100 \%$ \\
Stage Velocity & $1 \mathrm{~mm} / \mathrm{s}$ \\
Gate Delay & 0.5 \\
Gas & $1 \mathrm{~L} / \mathrm{min}$ Argon
\end{tabular}

Table 17.

Elements Detected in Non-Toxic Ammunitions by LIBS off SEM Stubs

\begin{tabular}{ll}
\hline Ammunition & Elemental \\
\hline Non-Toxic & \\
Fiocchi & $\mathrm{Al}, \mathrm{K}, \mathrm{Ca}$ \\
Syntech & $\mathrm{Al}, \mathrm{K}, \mathrm{Ba}$ \\
Inceptor & $\mathrm{K}, \mathrm{Ca}, \mathrm{Zn}$ \\
CCI & $\mathrm{Ca}, \mathrm{Ba}, \mathrm{Bi}$
\end{tabular}

\subsection{Conclusions}

The proposed mapping method using LIBS provides a rapid screening of elements in the gunshot residues deposited on the textile samples. For standard and leaded ammunitions, LIBS was able to detect whether lead, barium, and antimony were present, as well as their relative amounts per ammunition. Detection of additional elements, like copper from the cartridge case, were also detectable on the fabric samples shot by Remington, TulAmmo, Winchester, and Sellier and Bellot ammunitions. However, LIBS did not provide a full elemental profile of the gunshot residues left behind by the tested non-toxic ammunitions. Potassium, originating from potassium nitrate in the primer of non-toxic ammunition, and barium from the Syntech and CCX ammunition, were the only elements detected by LIBS when performing analysis directly on the clothing samples. SEM- 
EDS was able to detect a more comprehensive list of elements present in gunshot residue particles originating from both standard and non-toxic ammunitions. Moreover, once the residues were concentrated to the smaller area of an SEM stub, LIBS was able to detect additional elements that were also confirmed by SEM-EDS. The lack of detectability using LIBS suggests that the concentrations of some elements present, in each of the primers, are too low for LIBS to detect directly off clothing samples.

While this study provides proof of concept, limitations still exist in the testing of these clean ammunitions. Further research will focus on optimization of LIBS sampling methods to improve sensitivity and provide more accurate detections of modern ammunitions. 


\section{OVERALL CONCLUSIONS AND RECOMMENDATIONS}

\subsection{Conclusions of Proposed Methodologies}

In the last two years there was a reported total of 119,100 violent incidents involving a firearm. As of April 2019, there are already 17,001 incidents. These statistics stress the importance of efficient firearm discharge residue detection, observation, and analysis in forensic science. Accurate analysis of these residues can provide important investigative leads, corroborate stories, refute hypotheses, and help recreate the chain of events at a crime scene. Unfortunately, current methods used by practitioners lack the sensitivity, reliability, and versatility needed to accurately provide this information. Because of this, there is a crucial need for an enhanced detection method for firearm discharge residue evidence. This research project provided an alternative and practical solution using laser-induced breakdown spectroscopy.

LIBS proved to be a superior technique when predicting the true shooting distance of 35 unknown textile samples shot with conventional ammunition. Various background colors and patterns were chosen to represent clothing samples typically encountered at crime scenes that provide problems for conventional color testing. Using a single vertical ablation line, spectral and spatial information was obtained for over 100 known and unknown shooting distance samples. Subjecting this spectral data to multivariate classification methods, including regularized discriminant analysis, $100 \%$ of 35 unknown samples were classified as the correct distance range. On the other hand, conventional physical measurements and color tests correctly classified only $42.9 \%$ and $54.3 \%$ of samples, respectively. In other words, there was an average $51.4 \%$ increase in correct classifications when using LIBS to determine shooting distance compared to the conventional methods currently used by practitioners.

LIBS provided a simpler and more objective approach to bullet hole identification in surfaces continually encountered during firearm related incidents, including wood, glass, and drywall. A readily available adhesive was used to transfer and protect gunshot residues once removed from the surface. A rapid screening technique was able to detect gunshot residues directly around the bullet hole and $25 \mathrm{~mm}$ away from the bullet hole in under 30 minutes, without interference from the object itself. Conventional methods require harsh chemical methods to be applied in the field since labs are limited to bringing large objects in for analysis, or the objects are simply unmovable. The application of these methods in the field increases the chance of contamination and doesn't 
have the ability to avoid background interference such as paint or stains. Additionally, the LIBS method was non-destructive to the sample which allows for subsequent analyses by numerous methods, which is unlike color tests that damage the sample and limit the types of analyses that can be applied is sequence. While the rapid LIBS detection method showed some promise as an analytical tool to detect elements present in non-toxic ammunition, modifications to the method are still needed to improve the overall detection of modernized gunshot residues.

The LIBS methodologies proposed in this study aimed to bring more objective and accurate estimations for shooting distance determination, more confidence to bullet hole identification in large surfaces commonly encountered at crime scenes, and an additional detection method for nontoxic ammunitions that are becoming markedly more popular. Throughout the entirety of the project, laser induced breakdown spectroscopy proved to have numerous advantageous of conventional methods for gunshot residue detection, shooting distance determination, and bullet hole identification. First, the technique is virtually reagent-free, does not require sample preparation, and the micro-beam can be scanned across a target in a matter of minutes with minimal destruction to the sample. Second, the ablation platform can accommodate diverse substrates, and the laser settings can be controlled to offer different solutions, from ultra-fast screening for the identification of IGSR on entrance holes to detailed chemical mappings for shooting estimations and bullet hole identifications. Finally, LIBS offers a more reliable and objective presentation of the scientific results, leading to more reliable evidence to report in a courtroom.

The incorporation of these LIBS methodologies in forensic laboratories will help to modernize current practices and increase the scientific validity of the detection of gunshot residues in crime scene reconstruction. Additionally, with the advanced technology of portable instrumentation, there is the potential to incorporate this methodology into "on-site" field testing.

\subsection{Recommendations and Future Expansion of Work}

Based on the findings from the presented work, the following recommendations are proposed for future studies:

1. An extended sample size for shooting distance determination: textile samples of different fabric types, multiple different firearms and ammunitions, different amounts of blood used to soak the samples. 
2. Completing an inter-laboratory study using the proposed shooting distance determination to being transition into forensic laboratories for practitioner use.

3. A more complex list of substrates for bullet hole identification: painted drywall, stained wood, windshields, automobile doors.

4. Improvement of the sampling and LIBS method for the detection and identification of gunshot residue particles produced by non-toxic ammunitions, and utilizing other confirmatory methods such as y SEM-EDS and ICP-MS. Validating the method of using an SEM stub to pre-concentrate gunshot residue particles from substrates. 


\section{REFERENCES}

(1) Dalby, O.; Butler, D.; Birkett, J. W. Analysis of Gunshot Residue and Associated Materials - A Review. J. Forensic Sci. 2010, 55 (4), 924-943. https://doi.org/10.1111/j.15564029.2010.01370.x.

(2) Dockery, C. R.; Goode, S. R. Laser-Induced Breakdown Spectroscopy for the Detection of Gunshot Residues on the Hands of a Shooter. Appl. Opt. 2007, 42 (30), 6153. https://doi.org/10.1364/ao.42.006153.

(3) Schwoeble, A.; Exline, D. L. Current Methods in Gunshot Residue Analysis; 2000. https://doi.org/10.1017/CBO9781107415324.004.

(4) Wolten, G. M.; Jones, P. F.; Nesbitt, R. S.; Calloway, A. R.; Loper, G. L. Particle Analysis for the Detection of Gunshot Residue. I: Scanning Electron Microscopy/Energy Dispersive X-Ray Characterization of Hand Deposits from Firing. J. Forensic Sci. 2015, 24 (2), 10848J. https://doi.org/10.1520/jfs10848j.

(5) Mach, M. H.; Pallos, A.; Jones, P. F.; Maeh, M. H. Feasibility of Gunshot Residue Detection Via Its Organic Constituents; 1978; Vol. 23.

(6) Basu, S. Formation of Gunshot Residues. J. Forensic Sci. 2015, 27 (1), 11453J. https://doi.org/10.1520/jfs11453j.

(7) Newbury, D. E.; Ritchie, N. W. M.; Scott, J. H. J.; Joy, D. C.; Michael, J. R.; Goldstein, J. I. Scanning Electron Microscopy and X-Ray Microanalysis; 2017. https://doi.org/10.1007/9781-4939-6676-9.

(8) Nunziata, F.; Donghi, M. On the Formation of Basu's Type III (Peeled Orange) Gunshot Residues. Def. Technol. 2019, 15 (1), 23-26. https://doi.org/10.1016/j.dt.2018.09.002.

(9) ASTM. Standard Practice for Gunshot Residue Analysis by Scanning Electron Microscopy/Energy Dispersive X-Ray Spectrometry. Book of Standards. 2016, pp 1-5. https://doi.org/10.1520/E1588-17.

(10) MacCrehan, W. A.; Bedner, M. Development of a Smokeless Powder Reference Material for Propellant and Explosives Analysis. Forensic Sci. Int. 2006, 163 (1-2), 119-124. https://doi.org/10.1016/j.forsciint.2005.11.027.

(11) de Perre, C.; Corbin, I.; Blas, M.; McCord, B. R. Separation and Identification of Smokeless Gunpowder Additives by Capillary Electrochromatography. J. Chromatogr. A 2012, 1267, 259-265. https://doi.org/10.1016/j.chroma.2012.07.039.

(12) Nichok, R. Expectations Regarding Gunpowder Depositions. AFTE 1998, 30 (1), 94-101.

(13) Kusluski, M. A. The Physical Effects of Contact and Close-Distance Gunfire on Sweatshirt Fleece. J. Forensic Sci. 2018, 63 (3), 829-834. https://doi.org/10.1111/1556$\underline{4029.13619 .}$ 
(14) Stone, I. C.; DiMaio, V. J. M.; Petty, C. S. Gunshot Wounds: Visual and Analytical Procedures. J. Forensic Sci. 2015, 23 (2), 10769J. https://doi.org/10.1520/jfs10769j.

(15) Brożek-Mucha, Z. A Study of Gunshot Residue Distribution for Close-Range Shots with a Silenced Gun Using Optical and Scanning Electron Microscopy, X-Ray Microanalysis and Infrared Spectroscopy. Sci. Justice 2017, 57 (2), 87-94. https://doi.org/10.1016/j.scijus.2016.11.004.

(16) Izzharif, M.; Halim, A.; Kalthom, U.; Yew, C.; Koey, M. Analysis of Gunshot Residue Deposited on Cotton Cloth Target at Close Range Shooting Distances. J. Forensic Sci. 2010, 1 (1), 48-53.

(17) DiMaio, V. J. M.; Petty, C. S.; Stone, I. C. An Experimental Study of Powder Tattooing of the Skin. J. Forensic Sci. 2015, 21 (2), 10505J. https://doi.org/10.1520/jfs10505j.

(18) Joling, R. J.; Stern, W. W. An Overview of Firearms Identification Evidence for Attorneys. III: Qualifying and Using the Firearms Examiner as a Witness. J. Forensic Sci. 2015, 26 (1), 11343J. https://doi.org/10.1520/jfs11343j.

(19) Palenik, C. S.; Palenik, S.; Peter Diaczuk, L. Plumbum Microraptus: Definitive Microscopic Indicators of a Bullet Hole in a Synthetic Fabric*; 2013; Vol. 61.

(20) SWGGUN. Guidelines for Gunshot Residue Distance Determinations. 2013, pp 1-8.

(21) Dillon, J. The Modified Griess Test: A Chemically Specific Chromophoric Test for Nitrite Compounds in Gunshot Residues. AFTE 1990, 22 (3), 49-56.

(22) Glattstein, B.; Vinokurov, A.; Levin, N.; Zeichner, A. Improved Method for Shooting Distance Estimation. Part 1. Bullet Holes in Clothing Items. J. Forensic Sci. 2015, 45 (4), 14773J. https://doi.org/10.1520/jfs14773j.

(23) Berger, J.; Upton, C.; Springer, E. Evaluation of Total Nitrite Pattern Visualization as an Improved Method for Gunshot Residue Detection and Its Application to Casework Samples. J. Forensic Sci. 2019, 64 (1), 218-222. https://doi.org/10.1111/1556-4029.13802.

(24) Dillon, J. The Sodium Rhodizonate Test: A Chemically Specific Chromophoric Test for Lead in Gunshot Residues. AFTE 1990, 22 (3), 9-14.

(25) Bartsch, M. R.; Kobus, H. J.; Wainwright, K. P. An Update on the Use of the Sodium Rhodizonate Test for the Detection of Lead Originating from Firearm Discharges. J. Forensic Sci. 2015, 41 (6), 14047J. https://doi.org/10.1520/jfs14047j.

(26) Marty, W.; Sigrist, T.; Wyler, D. Determination of Firing Distance Using the Rhodizonate Staining Technique. Int. J. Legal Med. 2002, 116 (1), 1-4. https://doi.org/10.1007/s004140100220.

(27) Andreola, S.; Gentile, G.; Battistini, A.; Cattaneo, C.; Zoja, R. Forensic Applications of Sodium Rhodizonate and Hydrochloric Acid: A New Histological Technique for Detection of 
Gunshot Residues. J. Forensic Sci. 2011, 56 (3), 771-774. https://doi.org/10.1111/j.15564029.2010.01689.x.

(28) Lekstrom, J. A.; Koons, R. D. Copper and Nickel Detection on Gunshot Targets by Dithiooxamide Test. J. Forensic Sci. 2015, 31 (4), 11907J. https://doi.org/10.1520/jfs11907j.

(29) O'Mahony, A. M.; Wang, J. Electrochemical Detection of Gunshot Residue for Forensic Analysis: A Review. Electroanalysis 2013, 25 (6), 1341-1358.

https://doi.org/10.1002/elan.201300054.

(30) Management, G. S. Hydrocholoric Acid Safety Data Sheet. 2015, p 8.

(31) Scientific, T. Tartaric Acid Safety Data Sheet. 2010, pp 1-6.

(32) Scientific, T. Sulfanilic Acid Safety Data Sheet. 2014, pp 1-6.

(33) Scientific, T. Alpha-Naphthol Safety Data Sheet. 2015, pp 1-6.

(34) Management, G. S. Acetic Acid Safety Data Sheet. 2015, pp 1-8.

(35) Scientific, T. Sodium Nitrite Safety Data Sheet. Material safety data. 1999, pp 1-8. https://doi.org/10.1017/CBO9781107415324.004.

(36) Yuksel, B.; Ho, M.; Ovide, O.;Vander Pyl, C.; Trejos, T. Infrared Imaging As A Complementary Aid in Estimating Muzzle-to-Target Shooting Distance: An Application on Dark, Patterned and Bloody Sample. Turkiye Klin. J. Forensic Med. Forensic Sci. 2019. https://doi.org/10.5336/forensic.2019-64837.

(37) Atwater, C. S.; Durina, M. E.; Durina, J. P.; Blackledge, R. D. Visualization of Gunshot Residue Patterns on Dark Clothing. J. Forensic Sci. 2006, 51 (5), 1091-1095. https://doi.org/10.1111/j.1556-4029.2006.00226.x.

(38) Kersh, K. L.; Childers, J. M.; Justice, D.; Karim, G. Detection of Gunshot Residue on Dark-Colored Clothing Prior to Chemical Analysis. J. Forensic Sci. 2014, 59 (3), 754-762. https://doi.org/10.1111/1556-4029.12409.

(39) Popelka-Filcoff, R. S.; Kirkbride, K. P.; Seyfang, K. E.; Lucas, N.; Tucker, W. Gunshot Residue and Brakepads: Compositional and Morphological Considerations for Forensic Casework. Forensic Sci. Int. 2016, 270, 76-82. https://doi.org/10.1016/j.forsciint.2016.11.024.

(40) Ingo, G. M.; D'Uffizi, M.; Falso, G.; Bultrini, G.; Padeletti, G. Thermal and Microchemical Investigation of Automotive Brake Pad Wear Residues. In Thermochimica Acta; 2004; Vol. 418, pp 61-68. https://doi.org/10.1016/j.tca.2003.11.042.

(41) Vermeij, E.; Duvalois, W.; Webb, R.; Koeberg, M. Morphology and Composition of Pyrotechnic Residues Formed at Different Levels of Confinement. Forensic Sci. Int. 2009, 186 (1-3), 68-74. https://doi.org/10.1016/j.forsciint.2009.01.019. 
(42) Kosanke, K. L.; Dujay, R. C.; Kosanke, B. J. Pyrotechnic Reaction Residue Particle Analysis. J. Forensic Sci. 2006, 51 (2), 296-302. https://doi.org/10.1111/j.1556-

$\underline{4029.2006 .00055 . x}$.

(43) Phillips, S. Pyrotechnic Residues Analysis-Detection and Analysis of Characteristic Particles by SEM-EDS; 2001.

(44) Mosher, P. V.; McVicar, M. J.; Randall, E. D.; Sild, E. H. Gunshot Residue-Similar Particles Produced by Fireworks. J. Can. Soc. Forensic Sci. 1998, 31 (3), 157-168. https://doi.org/10.1080/00085030.1998.10757115.

(45) Grima, M.; Butler, M.; Hanson, R.; Mohameden, A. Firework Displays as Sources of Particles Similar to Gunshot Residue. Sci. Justice 2012, 52 (1), 49-57. https://doi.org/10.1016/j.scijus.2011.04.005.

(46) Berk, R. E. Automated SEM/EDS Analysis of Airbag Residue. II: Airbag Residue as a Source of Percussion Primer Residue Particles. J. Forensic Sci. 2009, 54 (1), 69-76. https://doi.org/10.1111/j.1556-4029.2008.00919.x.

(47) Garofano, L.; Capra, M.; Ferrari, F.; Bizzaro, G. P.; Di Tullio, D.; Dell'Olio, M.; Ghitti, A. Gunshot Residue. Forensic Sci. Int. 1999, 103 (1), 1-21. https://doi.org/10.1016/S0379$\underline{0738(99) 00035-3 .}$

(48) Bueno, J.; Sikirzhytski, V.; Lednev, I. K. Attenuated Total Reflectance-FT-IR Spectroscopy for Gunshot Residue Analysis: Potential for Ammunition Determination. Anal. Chem. 2013, 85 (15), 7287-7294. https://doi.org/10.1021/ac4011843.

(49) Sharma, S. P.; Lahiri, S. C. A Preliminary Investigation into the Use of FTIR Microscopy as a Probe for the Identification of Bullet Entrance Holes and the Distance of Firing. Sci. Justice 2009, 49 (3), 197-204. https://doi.org/10.1016/j.scijus.2008.07.002.

(50) Bueno, J.; Sikirzhytski, V.; Lednev, I. K. Raman Spectroscopic Analysis of Gunshot Residue Offering Great Potential for Caliber Differentiation. Anal. Chem. 2012, 84 (10), 4334 4339. https://doi.org/10.1021/ac203429x.

(51) Bueno, J.; Lednev, I. Adavanced Statistical Analysis and Discrimination of Gunshot Residue Implementing Combined Raman and FTIR Data. Anal. Methods 2013, 5 (22). https://doi.org/10.1039/c3ay40721g.

(52) Doty, K. C.; Lednev, I. K. Raman Spectroscopy for Forensic Purposes: Recent Applications for Serology and Gunshot Residue Analysis. TrAC - Trends Anal. Chem. 2018, 103, 215-222. https://doi.org/10.1016/j.trac.2017.12.003.

(53) Bueno, J.; Lednev, I. K. Raman Microspectroscopic Chemical Mapping and Chemometric Classification for the Identification of Gunshot Residue on Adhesive Tape. Anal. Bioanal. Chem. 2014, 406 (19), 4595-4599. https://doi.org/10.1007/s00216-014-7874-9. 
(54) Bueno, J.; Halámková, L.; Rzhevskii, A.; Lednev, I. K. Raman Microspectroscopic Mapping as a Tool for Detection of Gunshot Residue on Adhesive Tape. Anal. Bioanal. Chem. 2018, 410 (28), 7295-7303. https://doi.org/10.1007/s00216-018-1359-1.

(55) Mou, Y.; Lakadwar, J.; Rabalais, J. W. Evaluation of Shooting Distance by AFM and FTIR/ATR Analysis of GSR. J. Forensic Sci. 2008, 53 (6), 1381-1386. https://doi.org/10.1111/j.1556-4029.2008.00854.x.

(56) Gagliano-Candela, R.; Colucci, A. P.; Napoli, S. Determination of Firing Distance. Lead Analysis on the Target by Atomic Absorption Spectroscopy (AAS). J. Forensic Sci. 2008, 53 (2), 321-324. https://doi.org/10.1111/j.1556-4029.2008.00668.x.

(57) Schumacher, R.; Niewöhner, L.; Neimke, D.; Latzel, S.; Barth, M. Shooting Distance Determination by M-XRF-Examples on Spectra Interpretation and Range Estimation. Forensic Sci. Int. 2012, 223 (1-3), 273-278. https://doi.org/10.1016/j.forsciint.2012.10.001.

(58) Schumacher, R.; Barth, M.; Neimke, D.; Niewöhner, L. Investigation of Gunshot Residue Patterns Using Milli-XRF-Techniques: First Experiences in Casework. Scanning Microsc. 2010 2010, 7729 (June), 772917. https://doi.org/10.1117/12.853852.

(59) Reinhard Noll. Laser-Induced Breakdown Spectroscopy; 2012.

(60) Musazzi, S. LIBS Theory Application; 2014.

(61) Morgan, S. L.; Bachmeyer, M. F.; Dockery, C. R.; Nieuwland, A. A.; Goode, S. Detecting Gunshot Residue by Laser Induced Breakdown Spectroscopy. 2014, No. September, FB2. https://doi.org/10.1364/libs.2002.fb2.

(62) Silva, M. J.; Cortez, J.; Pasquini, C.; Honorato, R. S.; Paim, A. P. S.; Pimentel, M. F. Gunshot Residues: Screening Analysis by Laser-Induced Breakdown Spectroscopy. J. Braz. Chem. Soc. 2009, 20 (10), 1887-1894. https://doi.org/10.1590/S0103-50532009001000017.

(63) Rosenberg, M. B.; Dockery, C. R. Determining the Lifetime of Detectable Amounts of Gunshot Residue on the Hands of a Shooter Using Laser-Induced Breakdown Spectroscopy. Appl. Spectrosc. 2008, 62 (11), 1238-1241. https://doi.org/10.1366/000370208786401473.

(64) Hondrogiannis, E.; Andersen, D.; Miziolek, A. W. The Evaluation of a New Technology for Gunshot Residue (GSR) Analysis in the Field. Next-Generation Spectrosc. Technol. VI 2013, 8726 (May 2013), 87260P. https://doi.org/10.1117/12.2017900.

(65) Umpierrez, S.; Almirall, J. R.; Gornushkin, I.; Castro, W.; Winefordner, J. Forensic Elemental Analysis of Materials by Laser Induced Breakdown Spectroscopy (LIBS). Sensors, Command. Control. Commun. Intell. Technol. Homel. Secur. Homel. Def. IV 2005, 5778 (May 2005), 657. https://doi.org/10.1117/12.605754.

(66) Tarifa, A.; Almirall, J. R. Fast Detection and Characterization of Organic and Inorganic Gunshot Residues on the Hands of Suspects by CMV-GC-MS and LIBS. Sci. Justice 2015, 55 (3), 168-175. https://doi.org/10.1016/j.scijus.2015.02.003. 
(67) Trejos, T.; Vander Pyl, C.; Menking-Hoggatt, K.; Alvarado, A. L.; Arroyo, L. E. Fast Identification of Inorganic and Organic Gunshot Residues by LIBS and Electrochemical Methods. Forensic Chem. 2018, 8, 146-156. https://doi.org/10.1016/j.forc.2018.02.006.

(68) Hahn, D. W.; Omenetto, N. Laser-Induced Breakdown Spectroscopy (LIBS), Part II: Review of Instrumental and Methodological Approaches to Material Analysis and Applications to Different Fields. Appl. Spectrosc. 2012, 66 (4), 347-419. https://doi.org/10.1366/11-06574.

(69) López-López, M.; Alvarez-Llamas, C.; Pisonero, J.; García-Ruiz, C.; Bordel, N. An Exploratory Study of the Potential of LIBS for Visualizing Gunshot Residue Patterns. Forensic Sci. Int. 2017, 273, 124-131. https://doi.org/10.1016/j.forsciint.2017.02.012.

(70) Palleschi, V.; Schechter, I. Laser-Induced Breakdown Spectroscopy (LIBS):

Fundamentals and Applications; Miziolek, Ed.; Cambridge University.

(71) Tognoni, E.; Cristoforetti, G. Signal and Noise in Laser Induced Breakdown

Spectroscopy: An Introductory Review. Opt. Laser Technol. 2016, 79 (May), 164-172. https://doi.org/10.1016/j.optlastec.2015.12.010. 
APPENDIX I. ADDITIONAL PLOTS AND HEAT MAPS

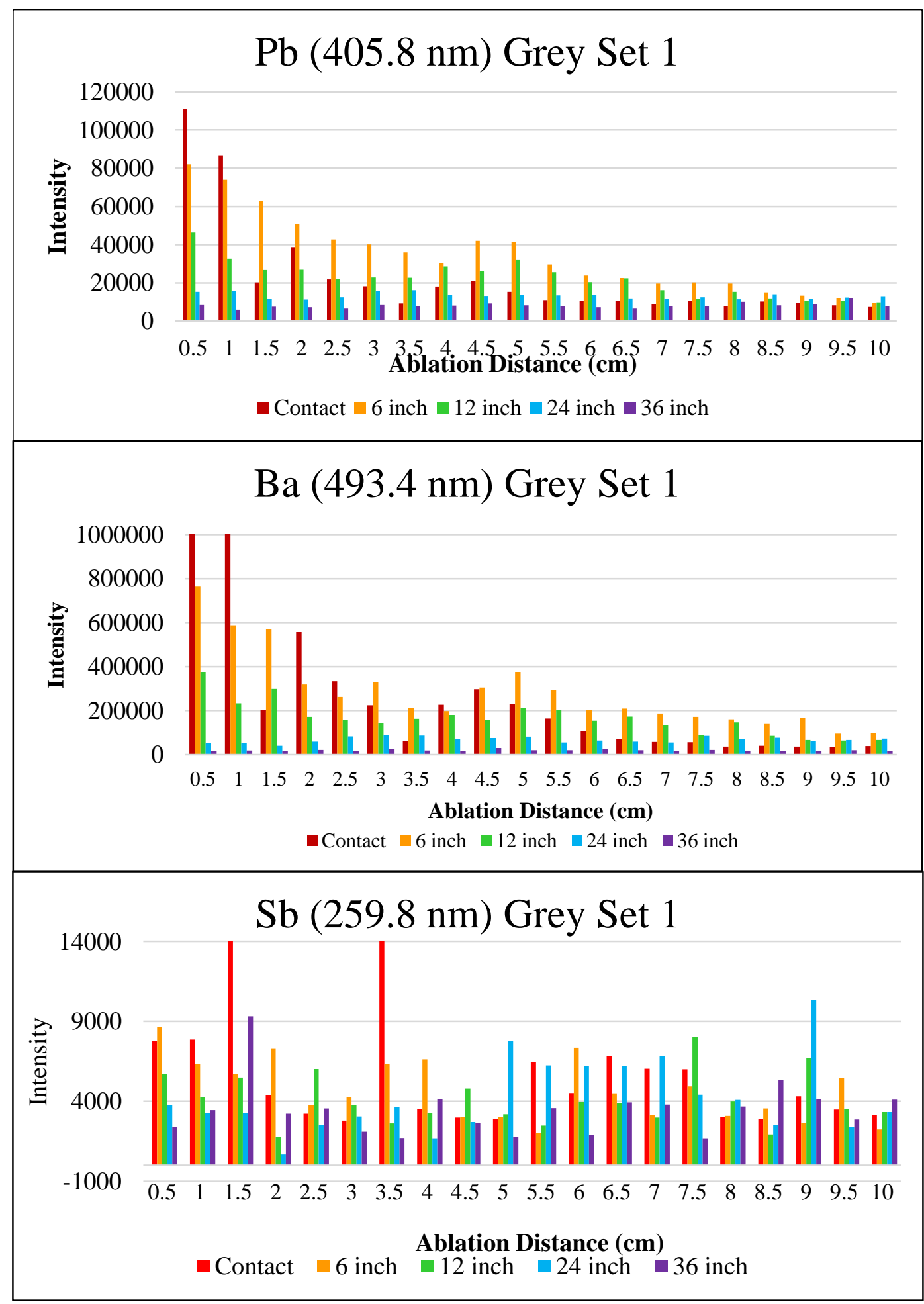

Figure 43. Bar graphs representing intensity patterns for $\mathrm{Pb}(405.8 \mathrm{~nm}), \mathrm{Ba}(493.4 \mathrm{~nm})$, and $\mathrm{Sb}(259.8 \mathrm{~nm})$ for grey fabric calibration set 1. From left to right, the ablation from the entrance hole begins at $0.5 \mathrm{~mm}$ and increases by $0.5 \mathrm{~mm}$ until ending at $100 \mathrm{~mm}$. The legend represents the different shooting distance used for calibration $($ Red $=$ Contact, Yellow $=6$ inches, Green $=12$ inches, Blue $=24$ inches, Purple $=36$ inches $)$. 


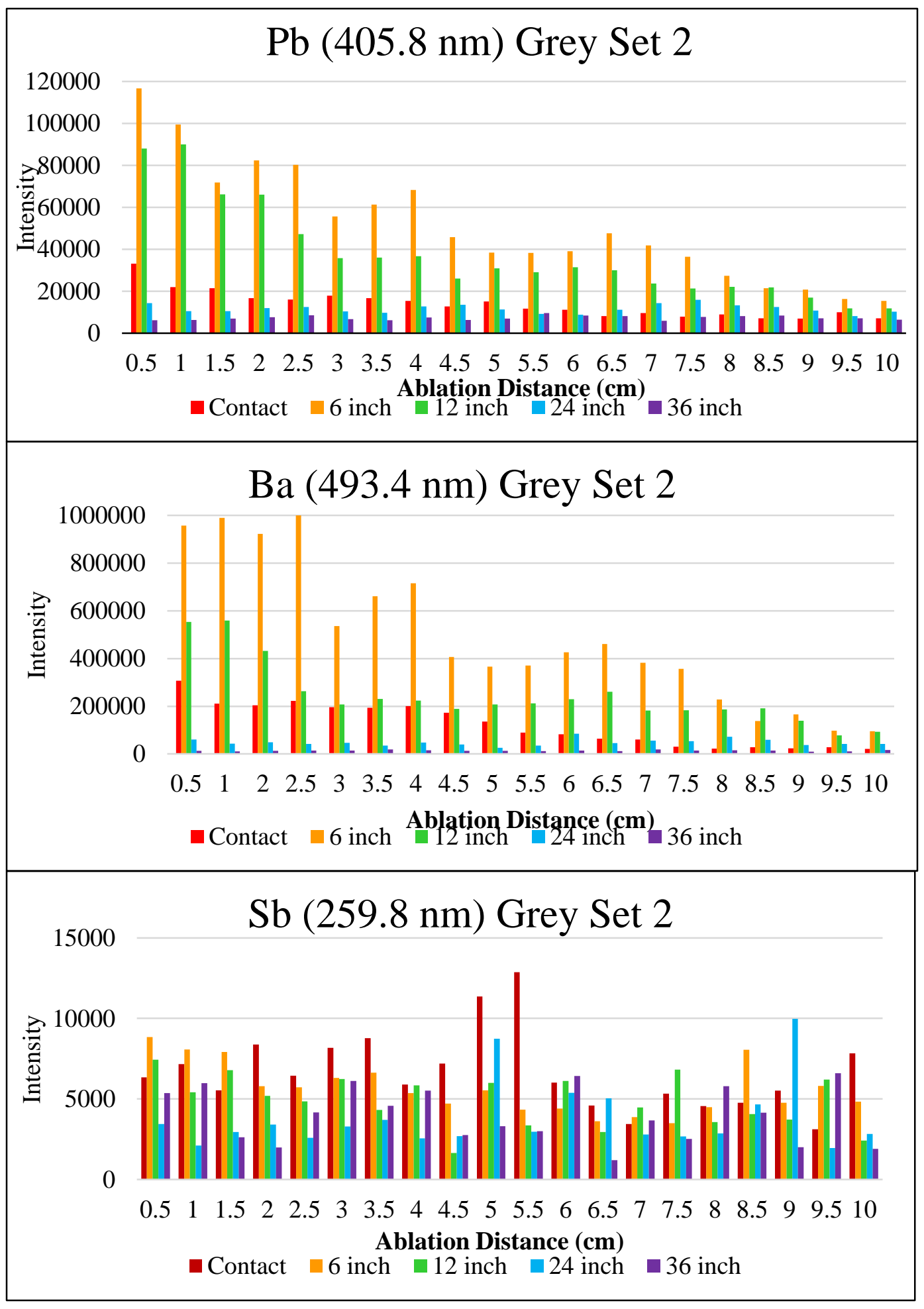

Figure 44. Bar graphs representing intensity patterns for $\mathrm{Pb}(405.8 \mathrm{~nm}), \mathrm{Ba}(493.4 \mathrm{~nm})$, and $\mathrm{Sb}(259.8 \mathrm{~nm})$ for grey fabric calibration set 2. From left to right, the ablation from the entrance hole begins at $0.5 \mathrm{~mm}$ and increases by 0.5 $\mathrm{mm}$ until ending at $100 \mathrm{~mm}$. The legend represents the different shooting distance used for calibration $(\mathrm{Red}=$ Contact, Yellow $=6$ inches, Green $=12$ inches, Blue $=24$ inches, Purple $=36$ inches. 


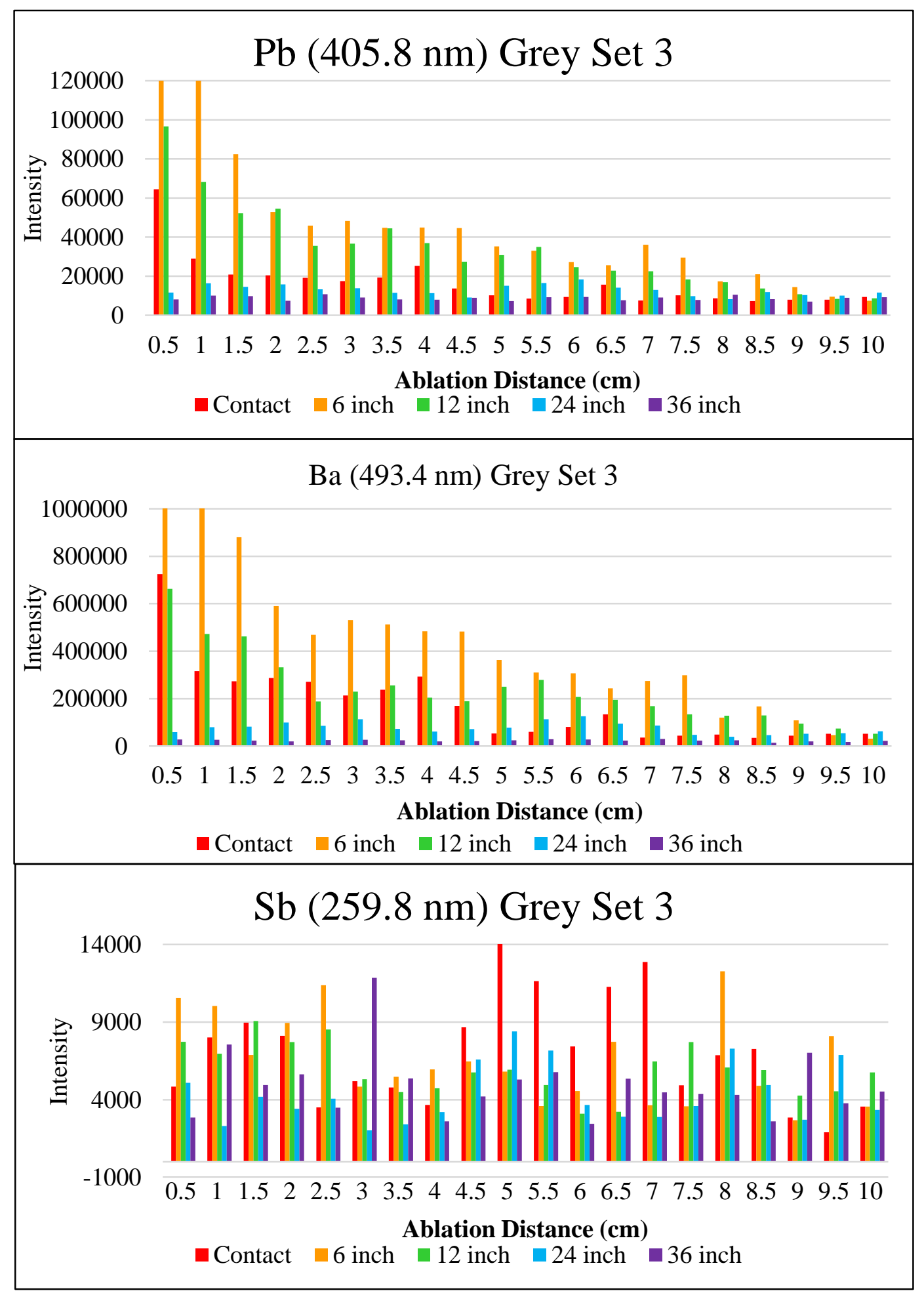

Figure 45. Bar graphs representing intensity patterns for $\mathrm{Pb}(405.8 \mathrm{~nm}), \mathrm{Ba}(493.4 \mathrm{~nm})$, and $\mathrm{Sb}(259.8 \mathrm{~nm})$ for grey fabric calibration set 3. From left to right, the ablation from the entrance hole begins at $0.5 \mathrm{~mm}$ and increases by 0.5 $\mathrm{mm}$ until ending at $100 \mathrm{~mm}$. The legend represents the different shooting distance used for calibration $(\mathrm{Red}=$ Contact, Yellow $=6$ inches, Green $=12$ inches, Blue $=24$ inches, Purple $=36$ inches. 


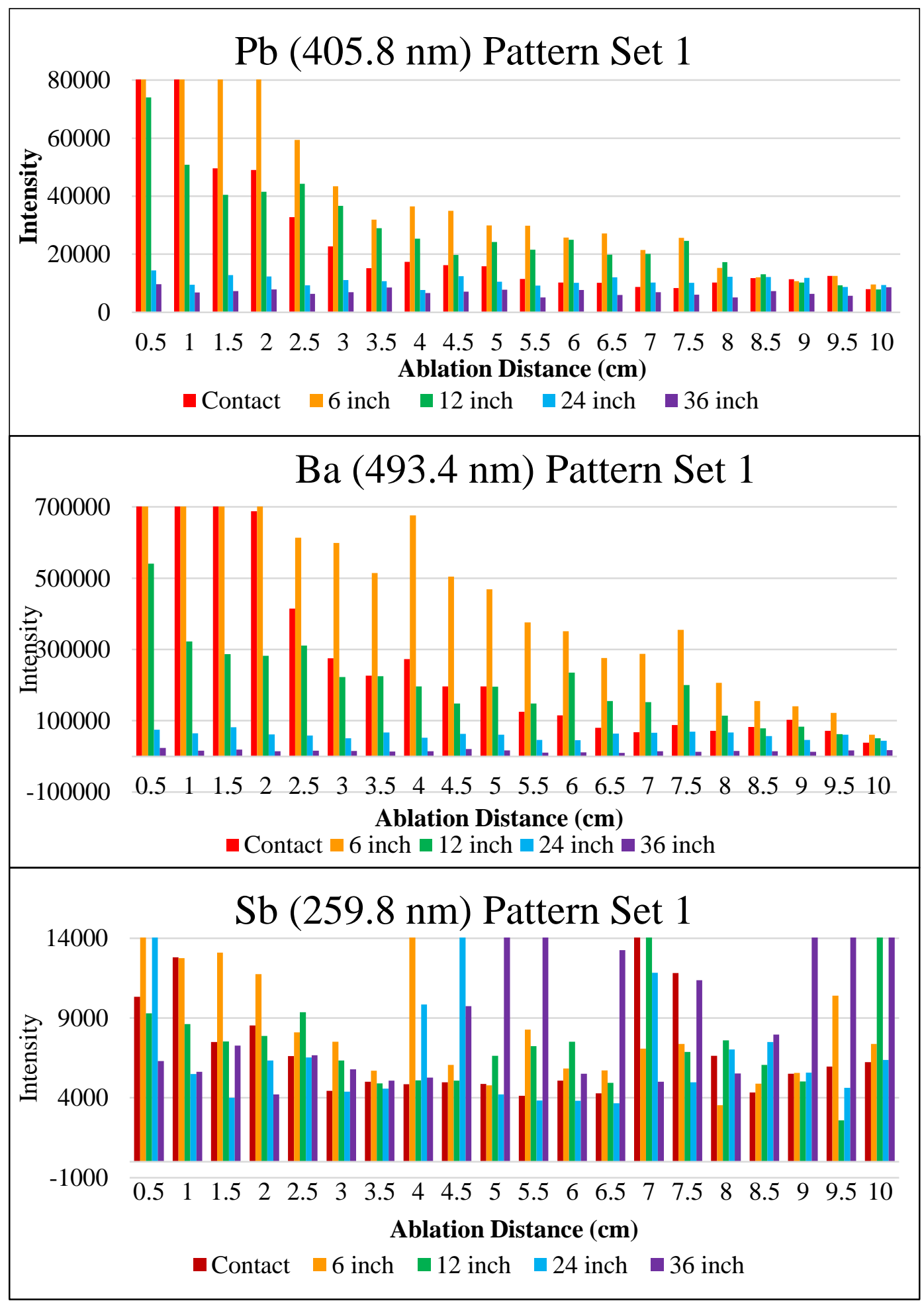

Figure 46. Bar graphs representing intensity patterns for $\mathrm{Pb}(405.8 \mathrm{~nm}), \mathrm{Ba}(493.4 \mathrm{~nm})$, and $\mathrm{Sb}(259.8 \mathrm{~nm})$ for patterned fabric calibration set 1 . From left to right, the ablation from the entrance hole begins at $0.5 \mathrm{~mm}$ and increases by $0.5 \mathrm{~mm}$ until ending at $100 \mathrm{~mm}$. The legend represents the different shooting distance used for calibration $($ Red $=$ Contact, Yellow $=6$ inches, Green $=12$ inches, Blue $=24$ inches, Purple $=36$ inches . 


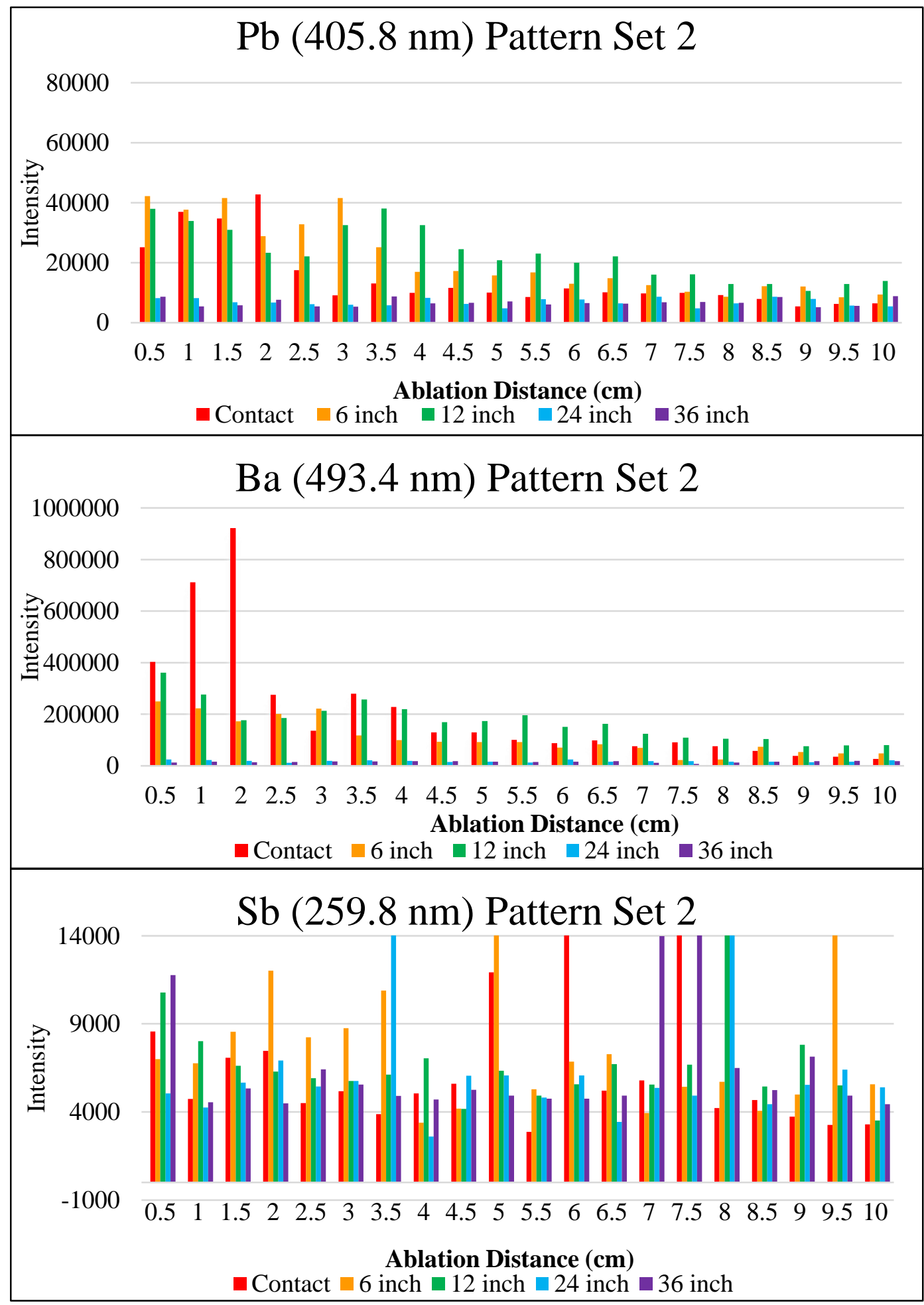

Figure 47. Bar graphs representing intensity patterns for $\mathrm{Pb}(405.8 \mathrm{~nm}), \mathrm{Ba}(493.4 \mathrm{~nm})$, and $\mathrm{Sb}(259.8 \mathrm{~nm})$ for patterned fabric calibration set 2. From left to right, the ablation from the entrance hole begins at $0.5 \mathrm{~mm}$ and increases by $0.5 \mathrm{~mm}$ until ending at $100 \mathrm{~mm}$. The legend represents the different shooting distance used for calibration $($ Red $=$ Contact, Yellow $=6$ inches, Green $=12$ inches, Blue $=24$ inches, Purple $=36$ inches. 


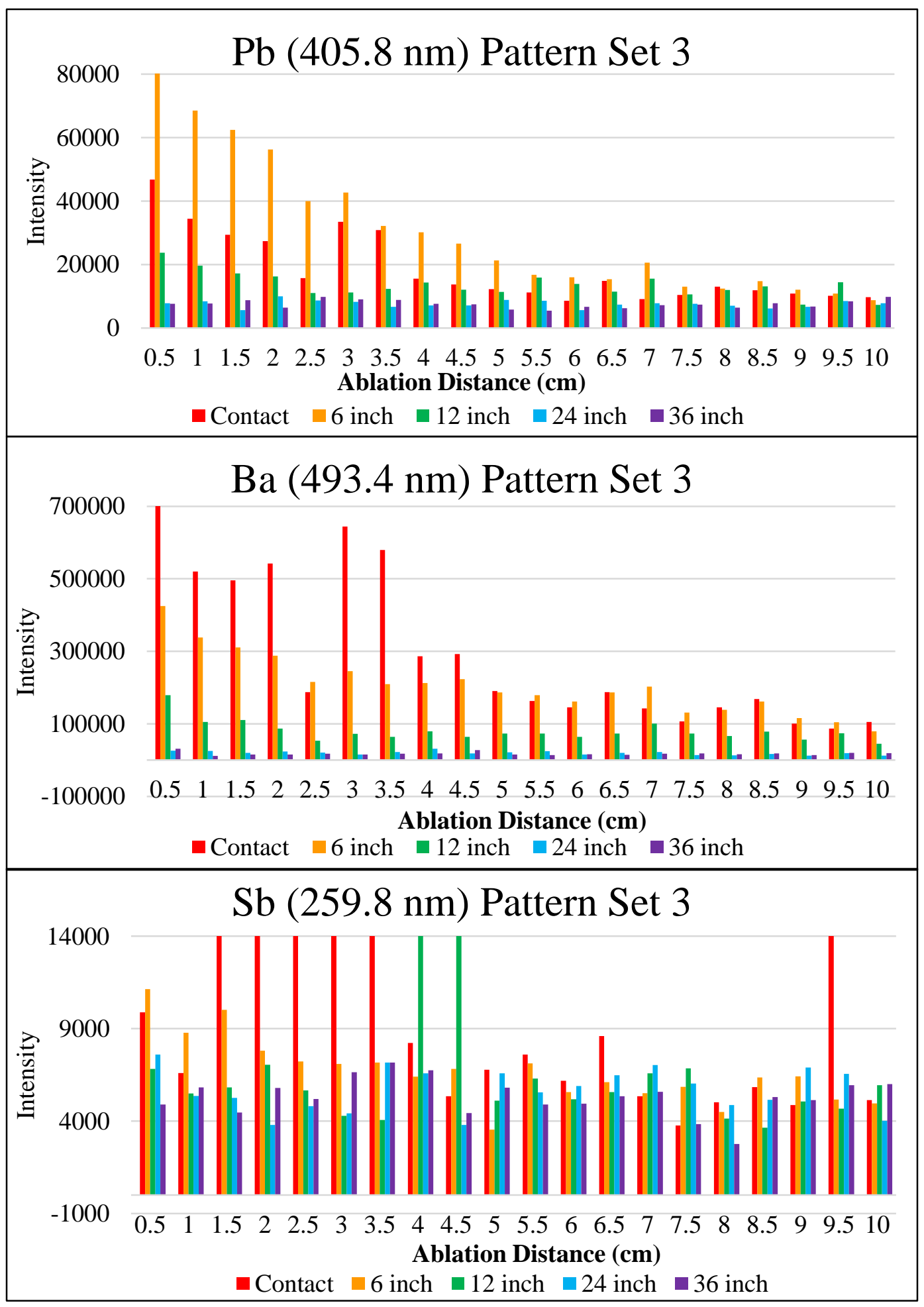

Figure 48. Bar graphs representing intensity patterns for $\mathrm{Pb}(405.8 \mathrm{~nm}), \mathrm{Ba}(493.4 \mathrm{~nm})$, and $\mathrm{Sb}(259.8 \mathrm{~nm})$ for patterned fabric calibration set 3. From left to right, the ablation from the entrance hole begins at $0.5 \mathrm{~mm}$ and increases by $0.5 \mathrm{~mm}$ until ending at $100 \mathrm{~mm}$. The legend represents the different shooting distance used for calibration $($ Red $=$ Contact, Yellow $=6$ inches, Green $=12$ inches, Blue $=24$ inches, Purple $=36$ inches. 


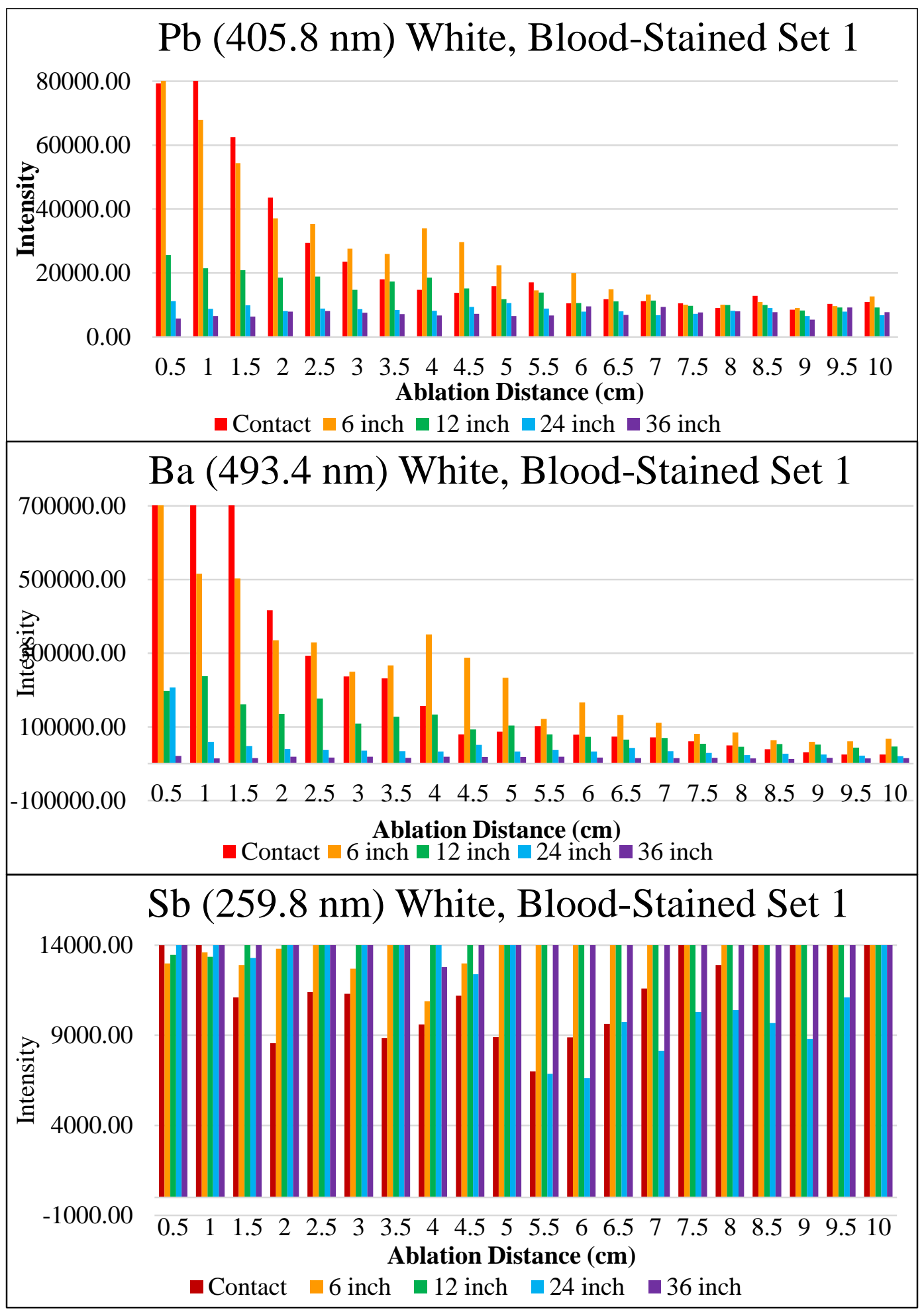

Figure 49. Bar graphs representing intensity patterns for $\mathrm{Pb}(405.8 \mathrm{~nm}), \mathrm{Ba}(493.4 \mathrm{~nm})$, and $\mathrm{Sb}(259.8 \mathrm{~nm})$ for blood-stained fabric calibration set 1 . From left to right, the ablation from the entrance hole begins at $0.5 \mathrm{~mm}$ and increases by $0.5 \mathrm{~mm}$ until ending at $100 \mathrm{~mm}$. The legend represents the different shooting distance used for calibration $($ Red $=$ Contact, Yellow $=6$ inches, Green $=12$ inches, Blue $=24$ inches, Purple $=36$ inches. 


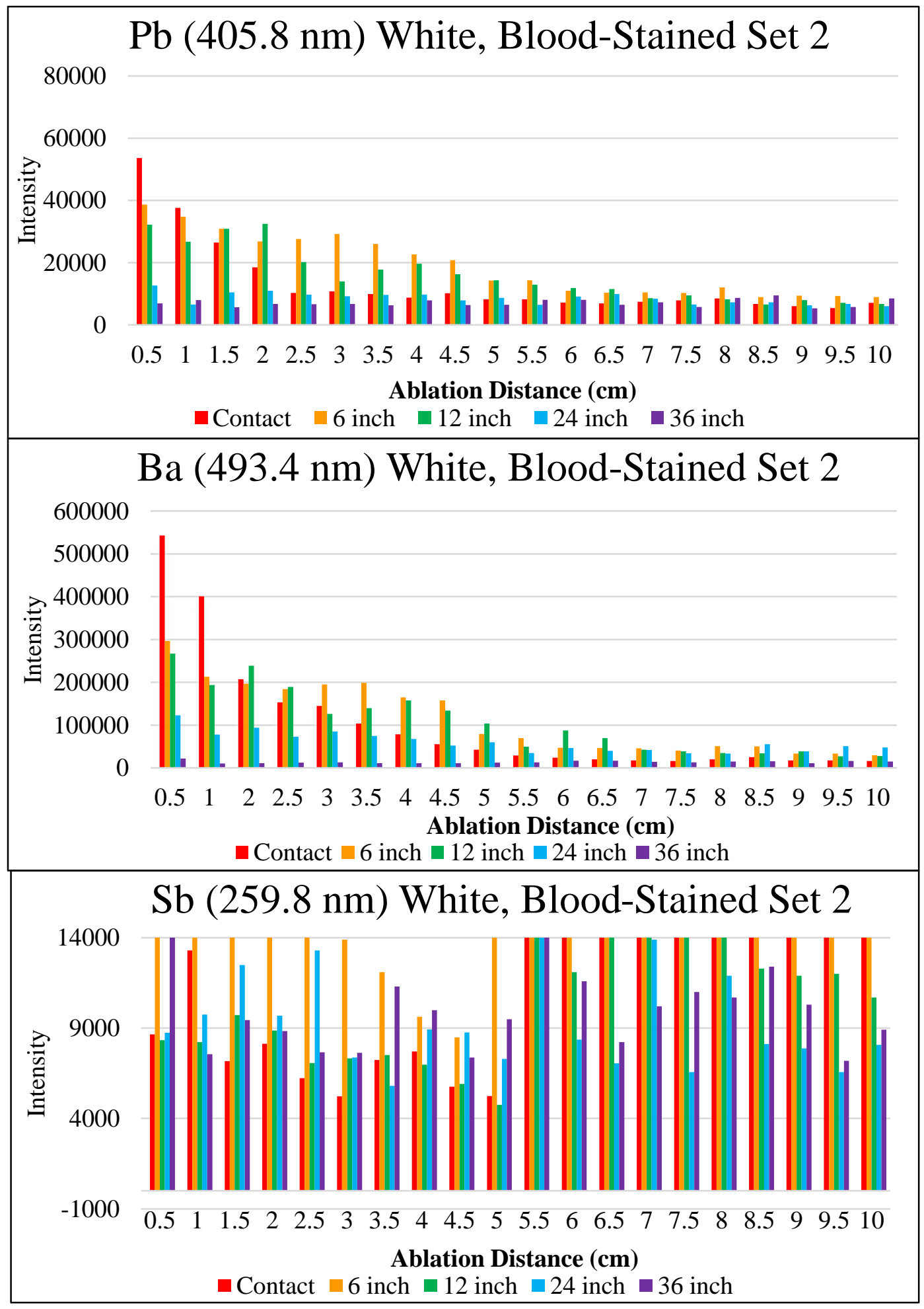

Figure 50. Bar graphs representing intensity patterns for $\mathrm{Pb}(405.8 \mathrm{~nm}), \mathrm{Ba}(493.4 \mathrm{~nm})$, and $\mathrm{Sb}(259.8 \mathrm{~nm})$ for blood-stained fabric calibration set 2 . From left to right, the ablation from the entrance hole begins at $0.5 \mathrm{~mm}$ and increases by $0.5 \mathrm{~mm}$ until ending at $100 \mathrm{~mm}$. The legend represents the different shooting distance used for calibration $($ Red $=$ Contact, Yellow $=6$ inches, Green $=12$ inches, Blue $=24$ inches, Purple $=36$ inches . 


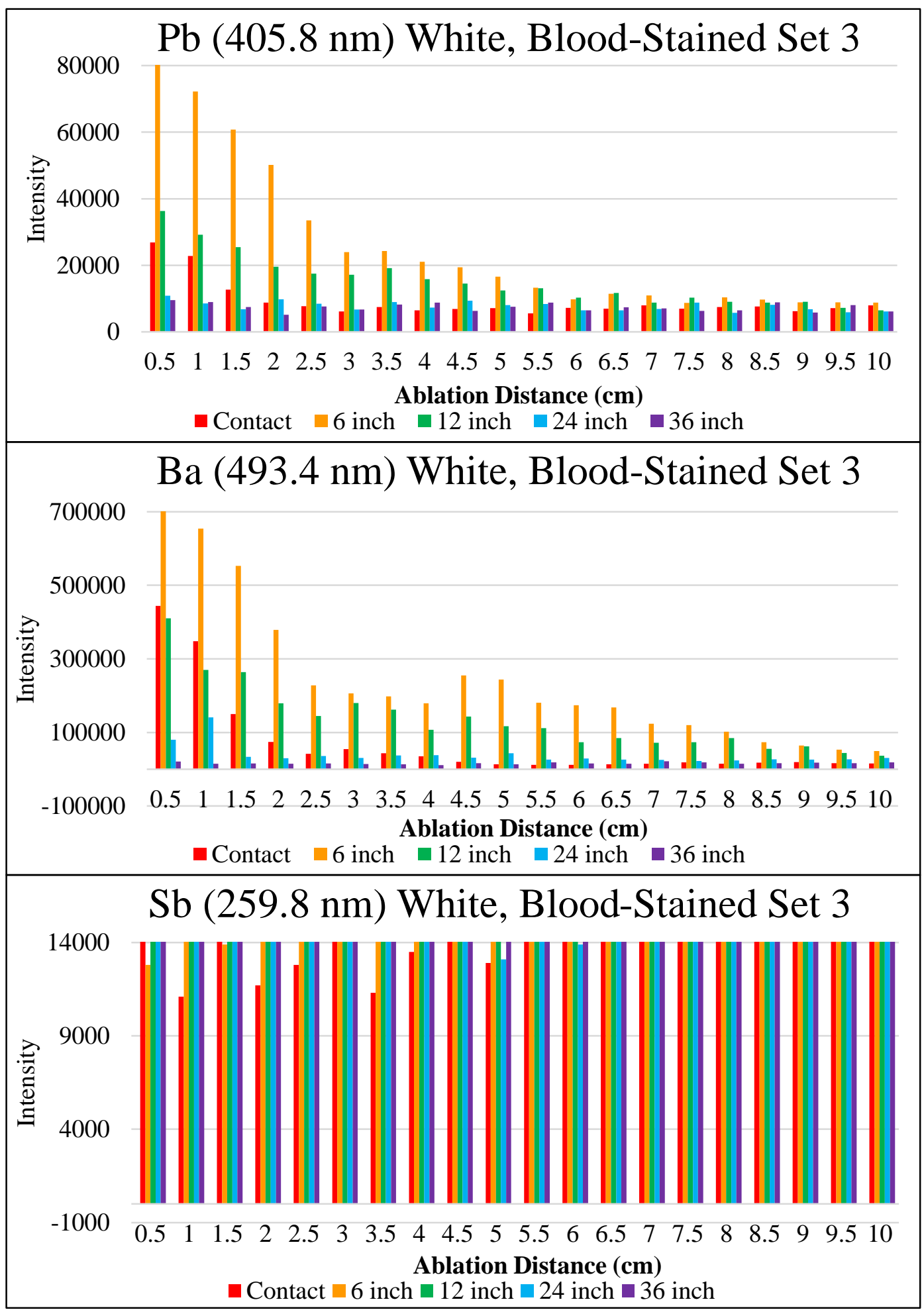

Figure 51. Bar graphs representing intensity patterns for $\mathrm{Pb}(405.8 \mathrm{~nm}), \mathrm{Ba}(493.4 \mathrm{~nm})$, and $\mathrm{Sb}(259.8 \mathrm{~nm})$ for blood-stained fabric calibration set 3 . From left to right, the ablation from the entrance hole begins at $0.5 \mathrm{~mm}$ and increases by $0.5 \mathrm{~mm}$ until ending at $100 \mathrm{~mm}$. The legend represents the different shooting distance used for calibration $($ Red $=$ Contact, Yellow $=6$ inches, Green $=12$ inches, Blue $=24$ inches, Purple $=36$ inches . 

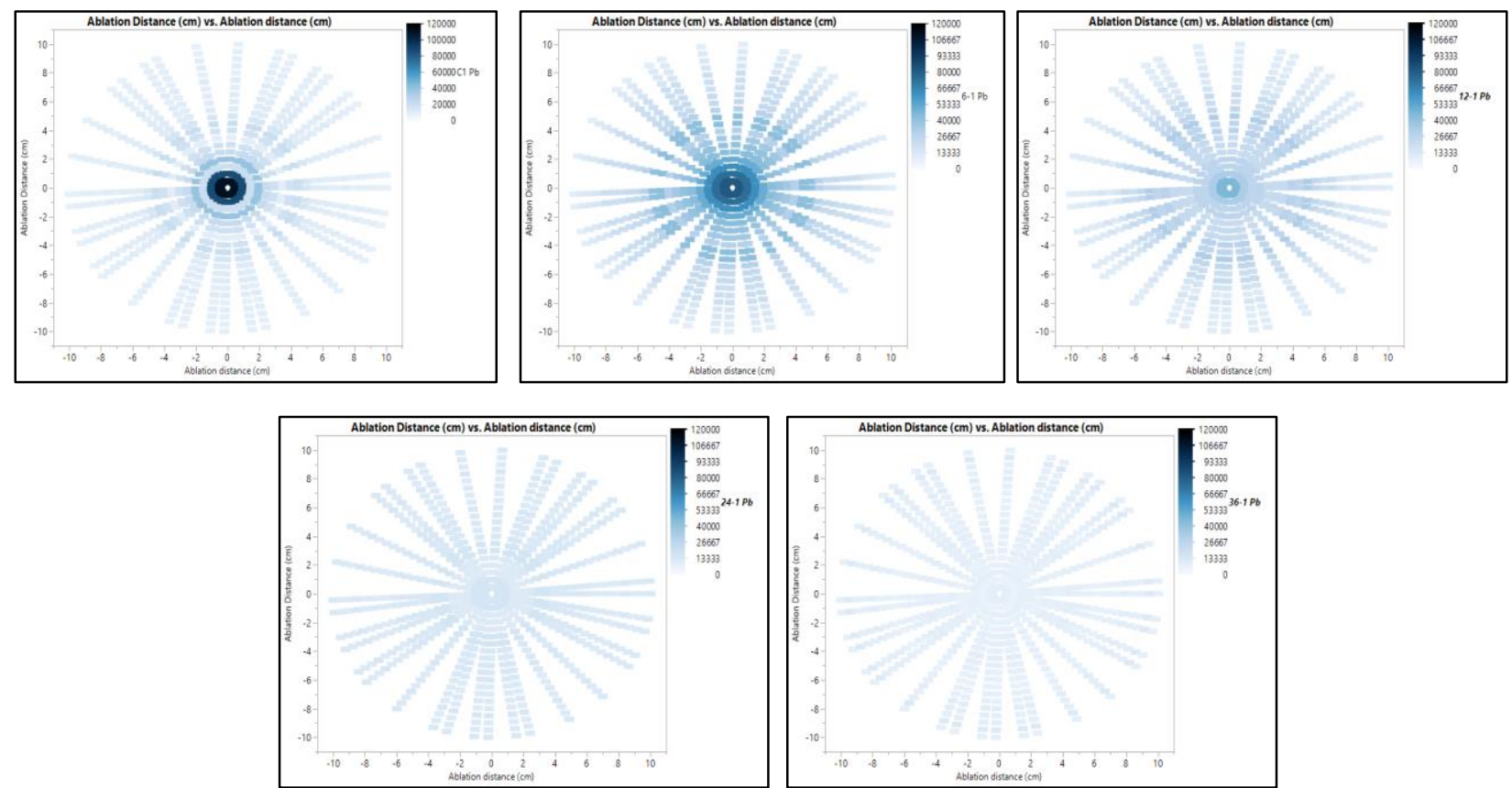

Figure 52. Heat maps of $\mathrm{Pb}(405.8 \mathrm{~nm})$ obtained from grey calibration set number 1; From Left to Right: contact, 6 inches, 12 inches, 24 inches, 36 inches.
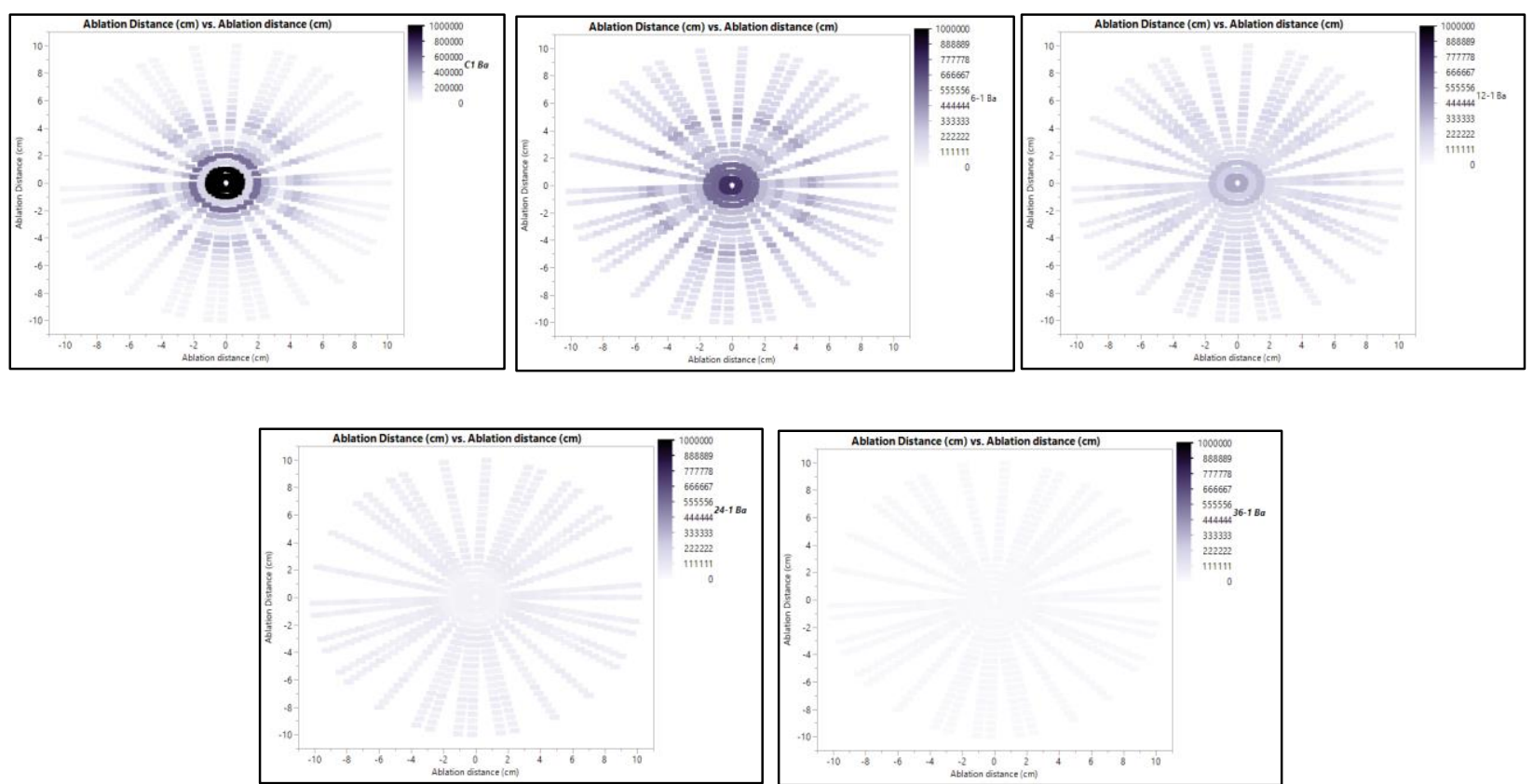

Figure 53. Heat maps of $\mathrm{Ba}(493.4 \mathrm{~nm})$ obtained from grey calibration set number 1; From Left to Right: contact, 6 inches, 12 inches, 24 inches, 36 inches. 

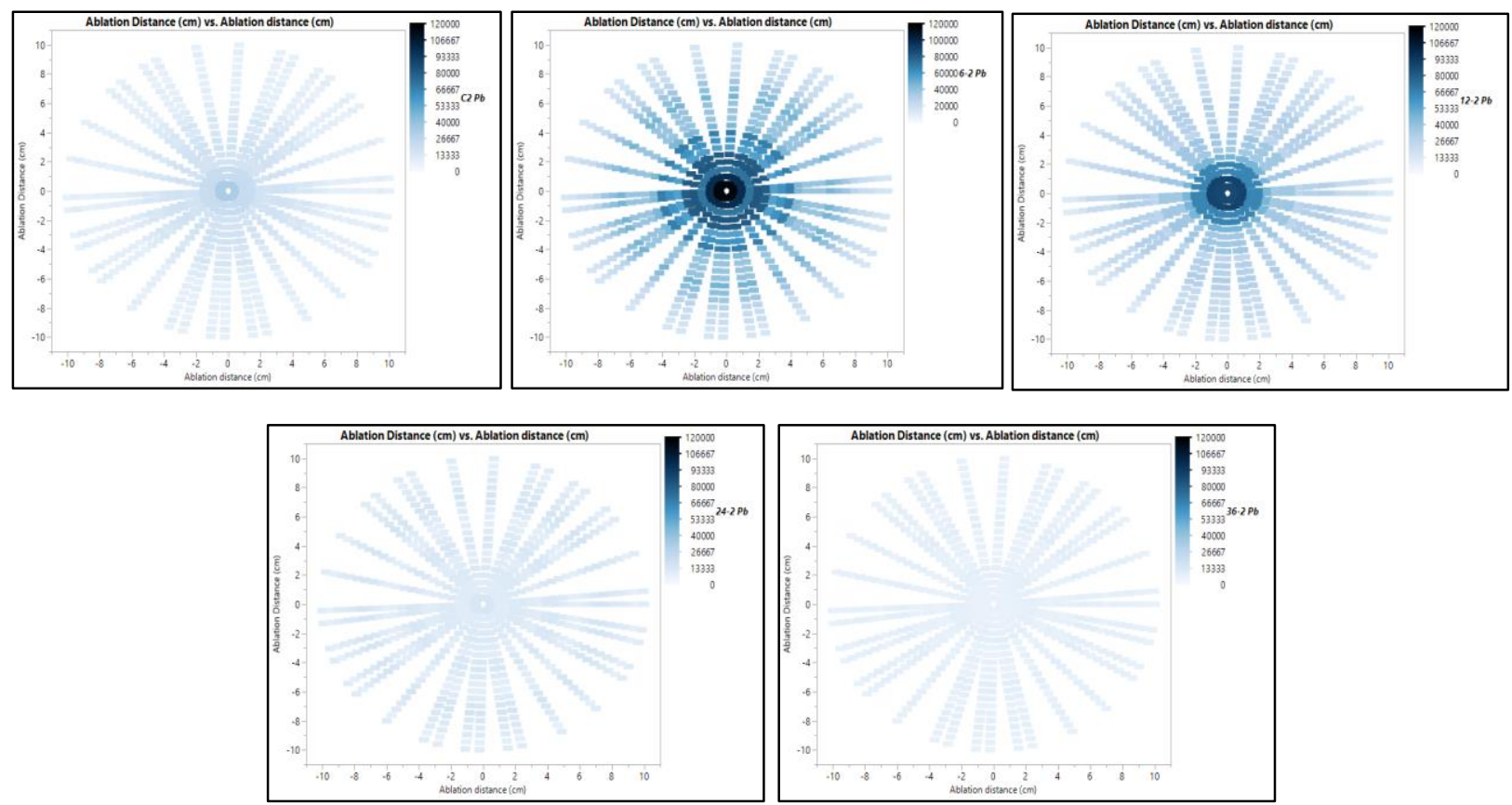

Figure 54. Heat maps of $\mathrm{Pb}(405.8 \mathrm{~nm})$ obtained from grey calibration set number 2; From Left to Right: contact, 6 inches, 12 inches, 24 inches, 36 inches.
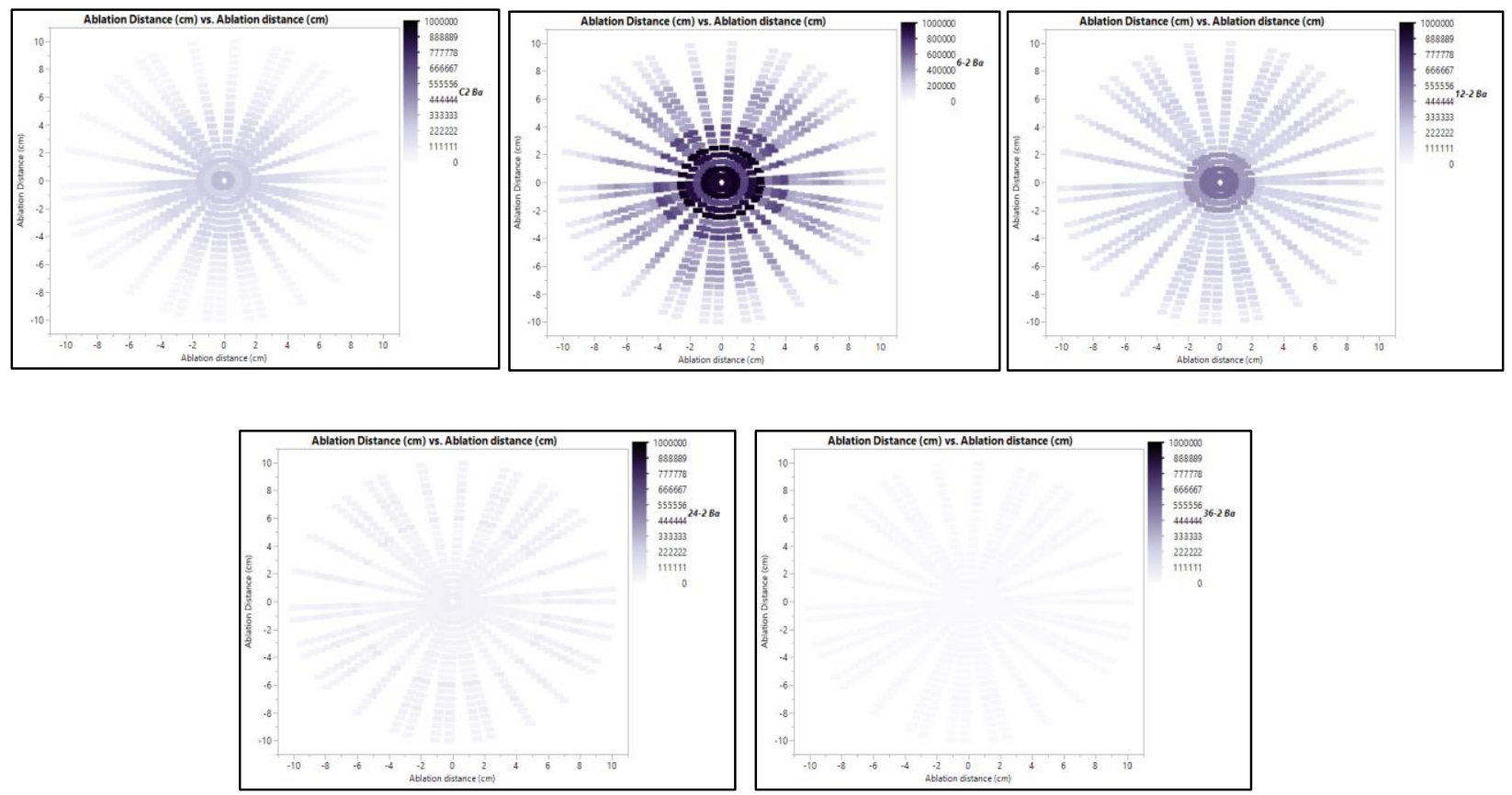

Figure 55. Heat maps of $\mathrm{Ba}(493.4 \mathrm{~nm})$ obtained from grey calibration set number 2; From Left to Right: contact, 6 inches, 12 inches, 24 inches, 36 inches. 

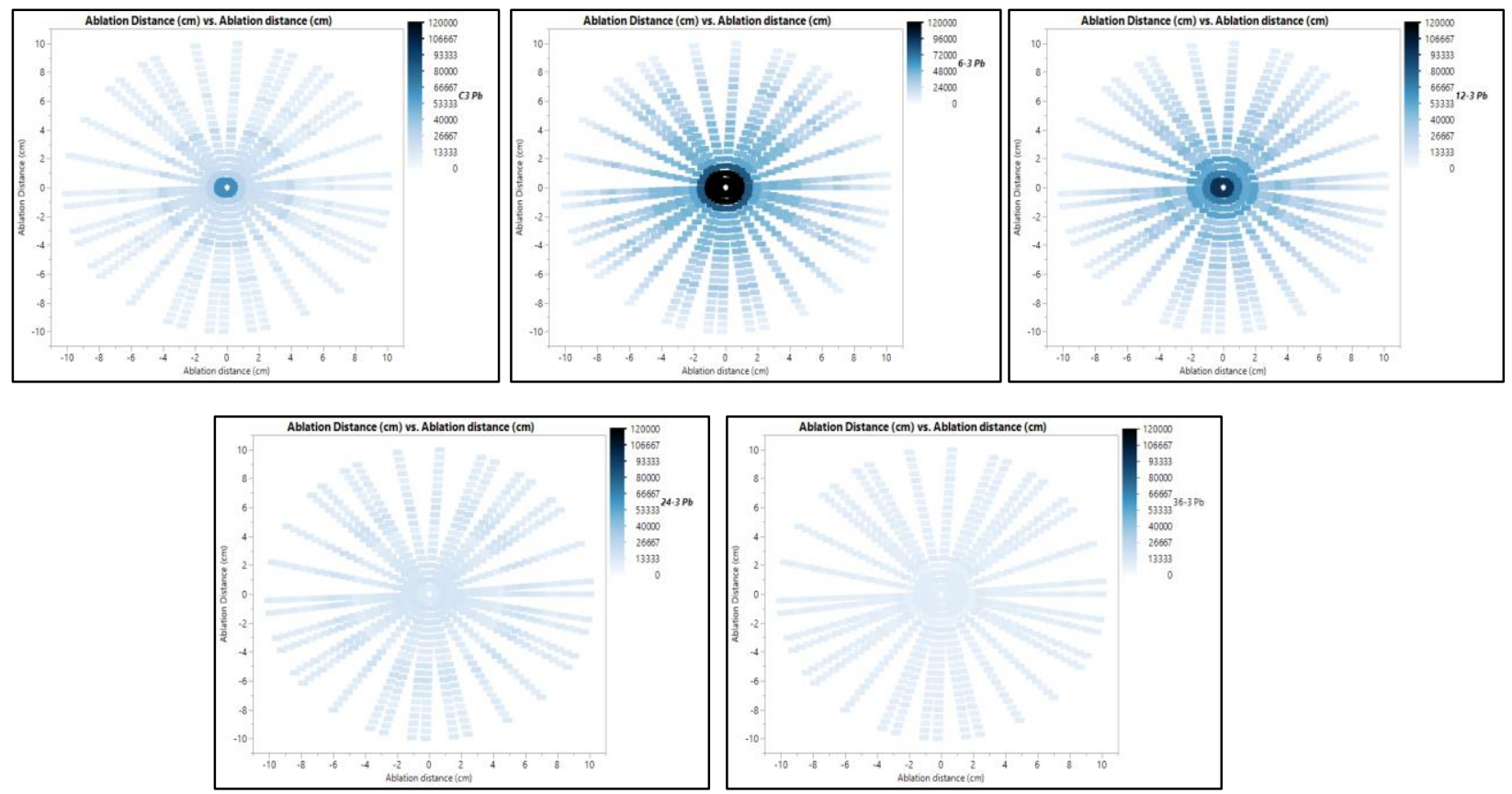

Figure 56. Heat maps of $\mathrm{Pb}(405.8 \mathrm{~nm})$ obtained from grey calibration set number 3; From Left to Right: contact, 6 inches, 12 inches, 24 inches, 36 inches.
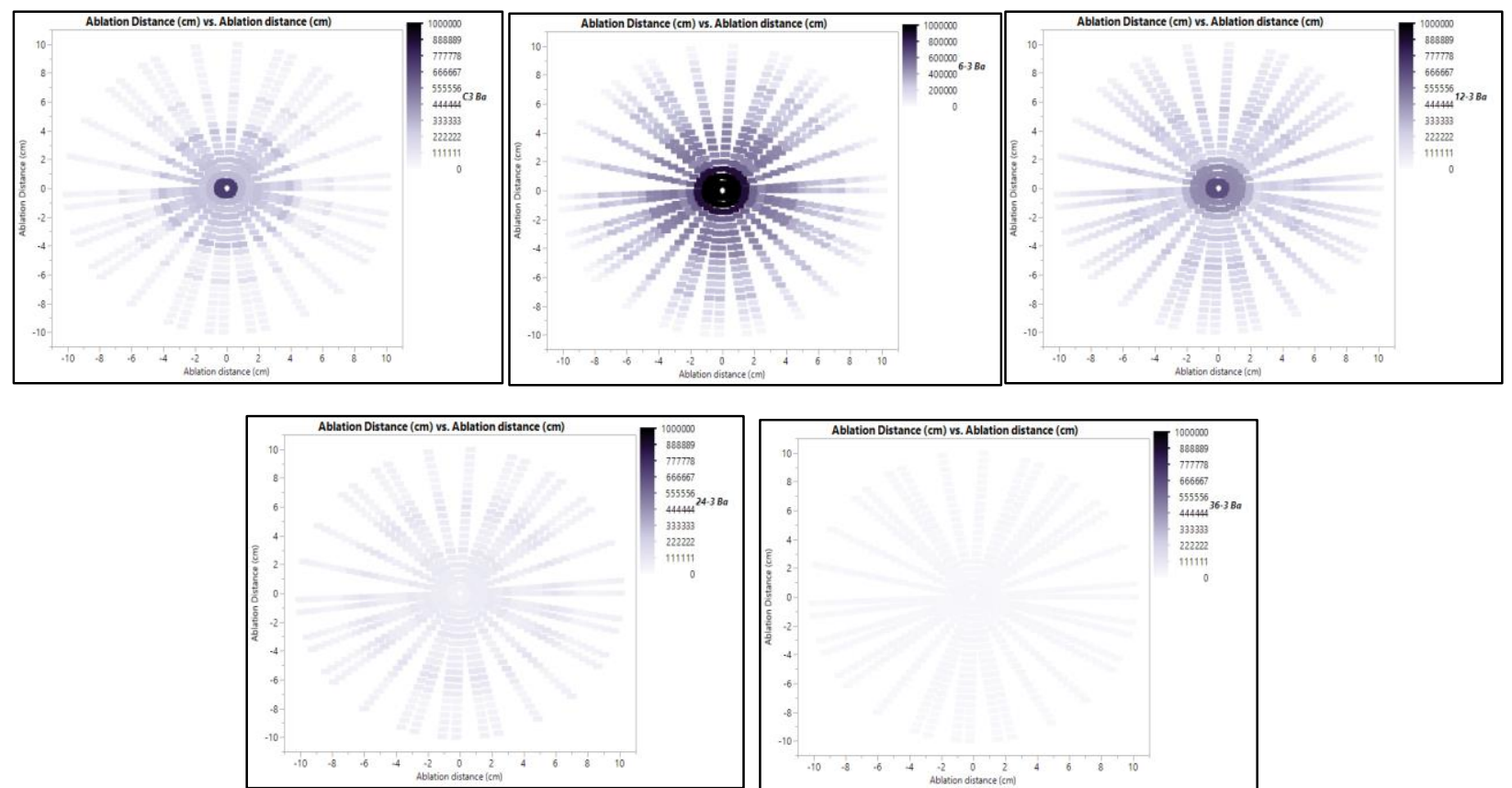

Figure 57. Heat maps of $\mathrm{Ba}(493.4 \mathrm{~nm})$ obtained from grey calibration set number 3; From Left to Right: contact, 6 inches, 12 inches, 24 inches, 36 inches. 

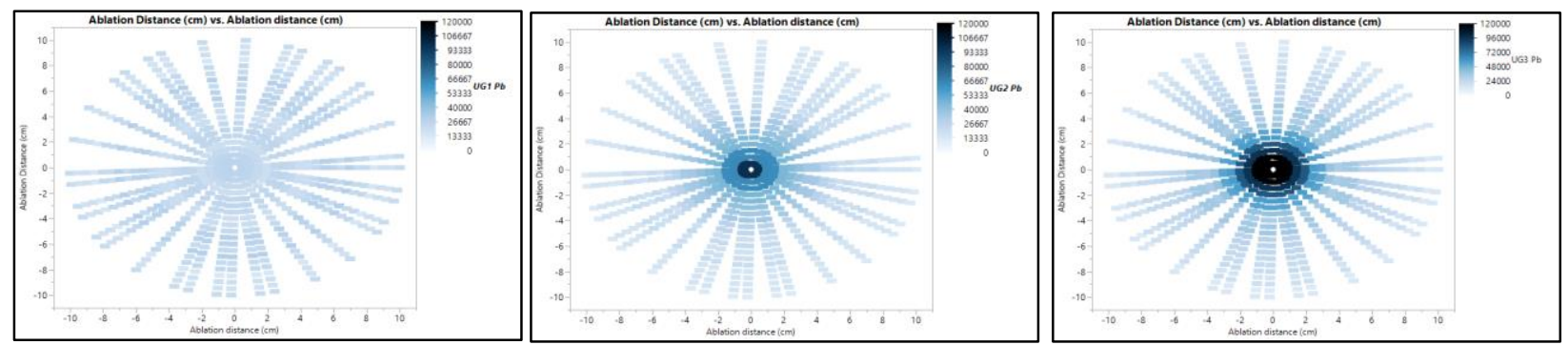

Figure 58. Heat maps of $\mathrm{Pb}(405.8 \mathrm{~nm})$ obtained from grey unknowns; From Left to Right: grey unknown 1, grey unknown 2 , grey unknown 3.
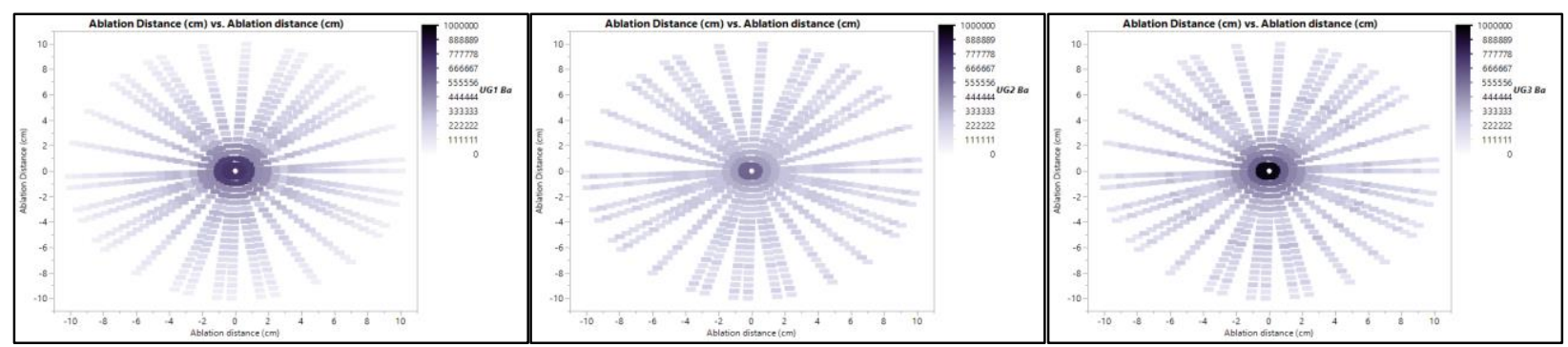

Figure 59. Heat maps of $\mathrm{Ba}(493.4 \mathrm{~nm})$ obtained from grey unknowns; From Left to Right: grey unknown 1, grey unknown 2, grey unknown 3.
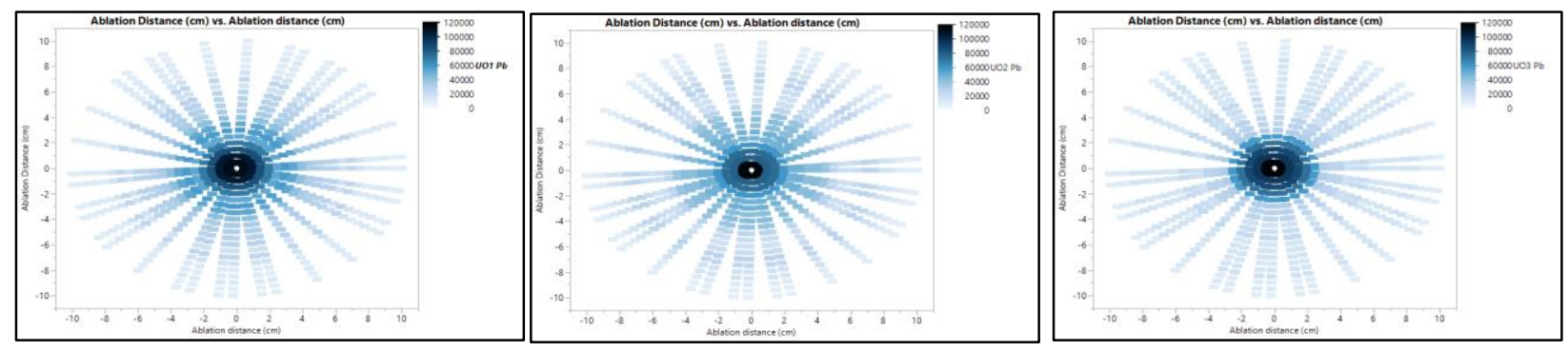

Figure 60. Heat maps of $\mathrm{Pb}(405.8 \mathrm{~nm})$ obtained from orange unknowns; From Left to Right: orange unknown 1, orange unknown 2, orange unknown 3.

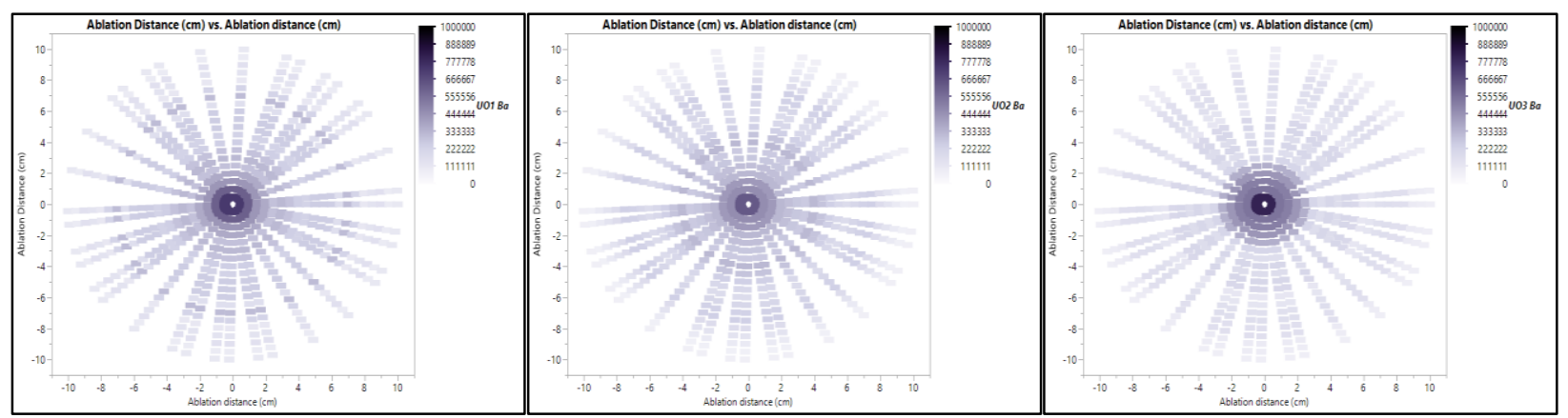

Figure 61. Heat maps of Ba (493.4 nm) obtained from orange unknowns; From Left to Right: orange unknown 1, orange unknown 2, orange unknown 3. 

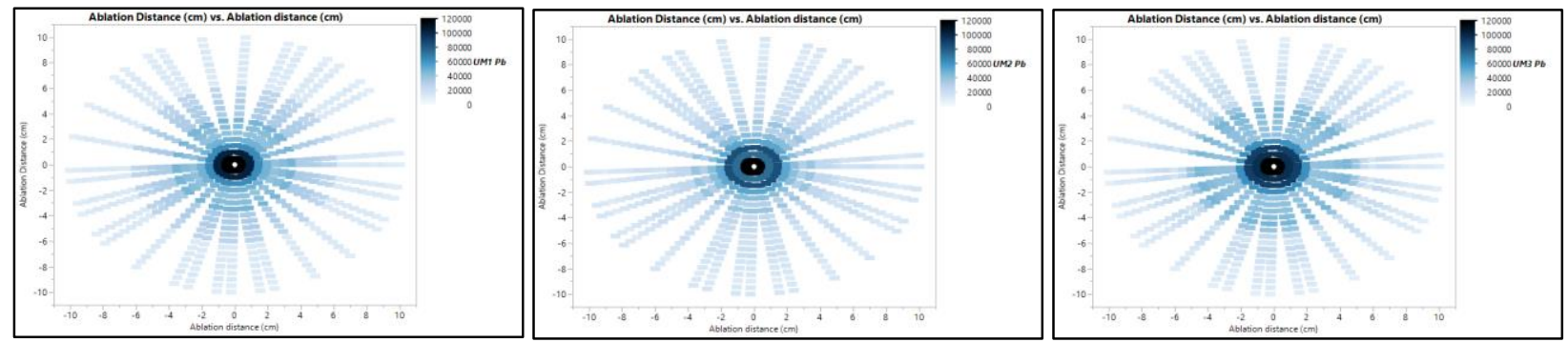

Figure 62. Heat maps of $\mathrm{Pb}(405.8 \mathrm{~nm})$ obtained from maroon unknowns; From Left to Right: maroon unknown 1, maroon unknown 2, maroon unknown 3.

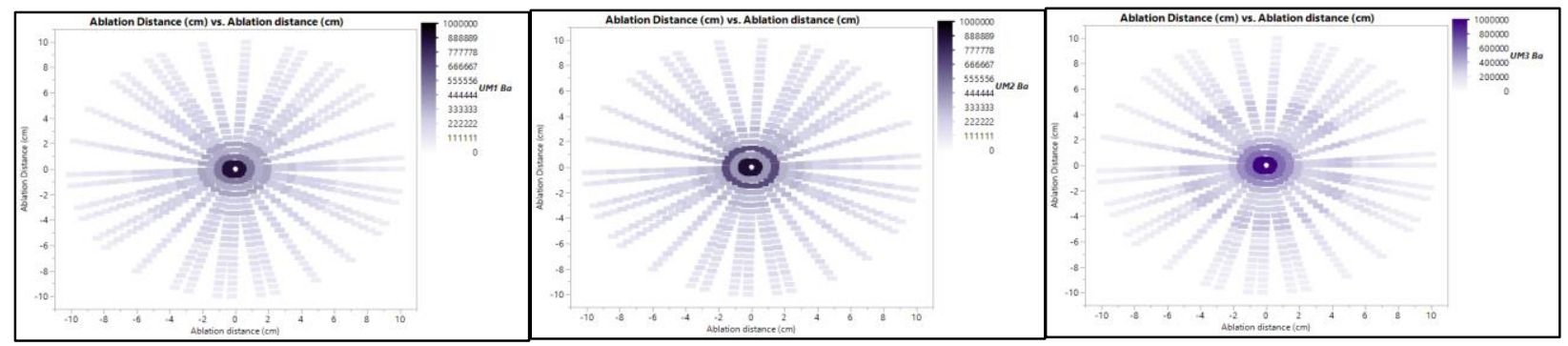

Figure 63. Heat maps of $\mathrm{Ba}$ (493.4) obtained from maroon unknowns; From Left to Right: maroon unknown 1, maroon unknown 2, maroon unknown 3.
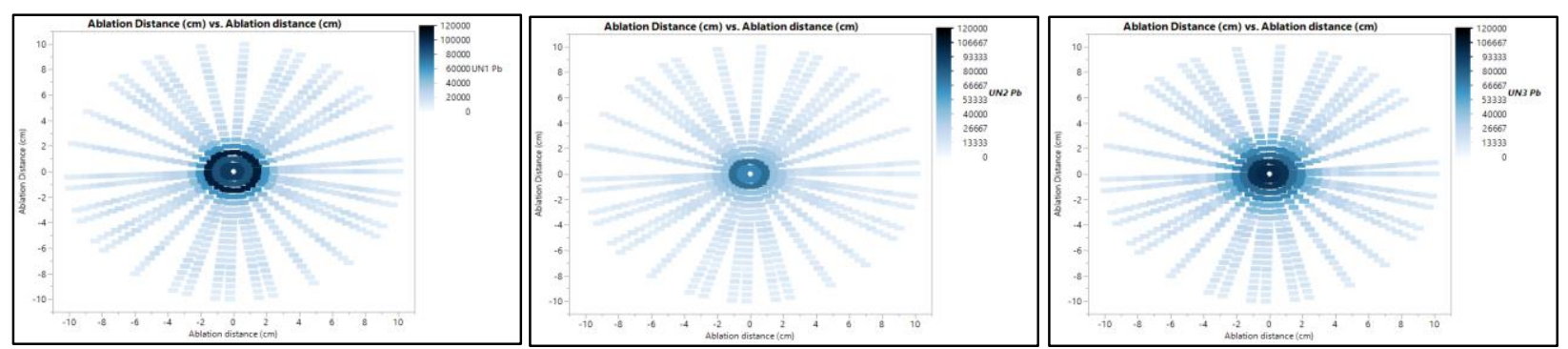

Figure 64. Heat maps of $\mathrm{Pb}(405.8 \mathrm{~nm})$ obtained from navy unknowns; From Left to Right: navy unknown 1, navy unknown 2, navy unknown 3.

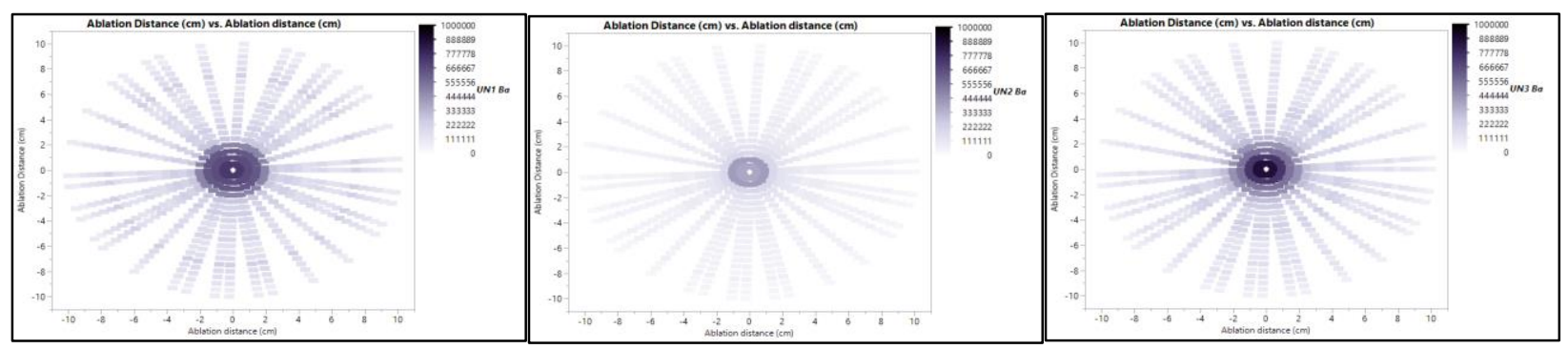

Figure 65. Heat maps of $\mathrm{Pb}(405.8 \mathrm{~nm})$ obtained from navy unknowns; From Left to Right: navy unknown 1, navy unknown 2, navy unknown 3. 

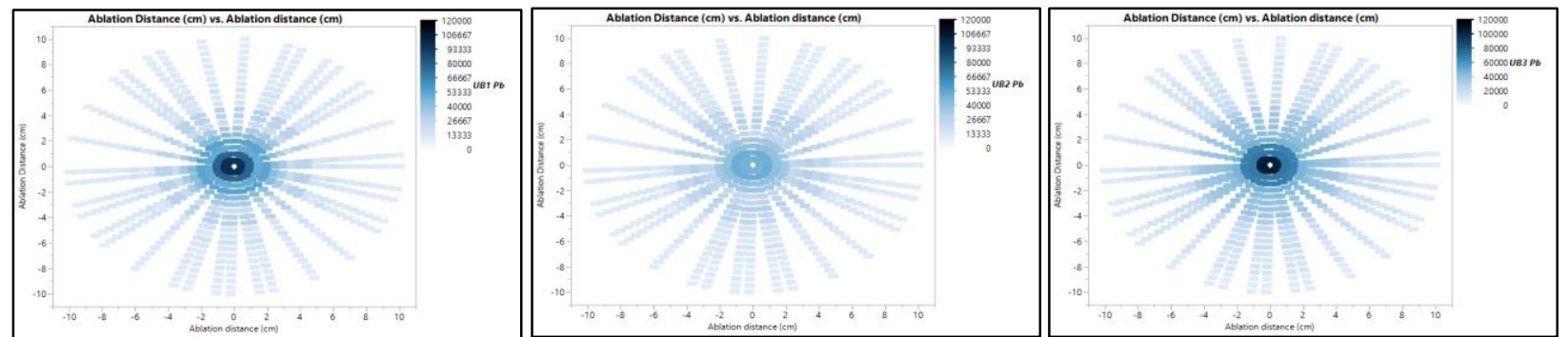

Figure 66. Heat maps of $\mathrm{Pb}(405.8 \mathrm{~nm})$ obtained from black unknowns; From Left to Right: black unknown 1, black unknown 2, black unknown 3 .

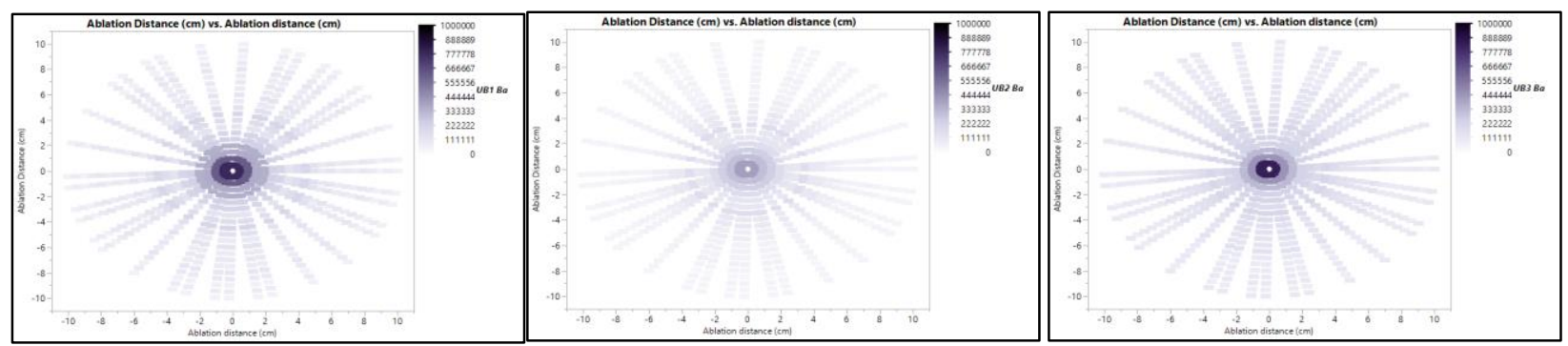

Figure 67. Heat maps of Ba (493.4 nm) obtained from black unknowns; From Left to Right: black unknown 1, black unknown 2, black unknown 3.
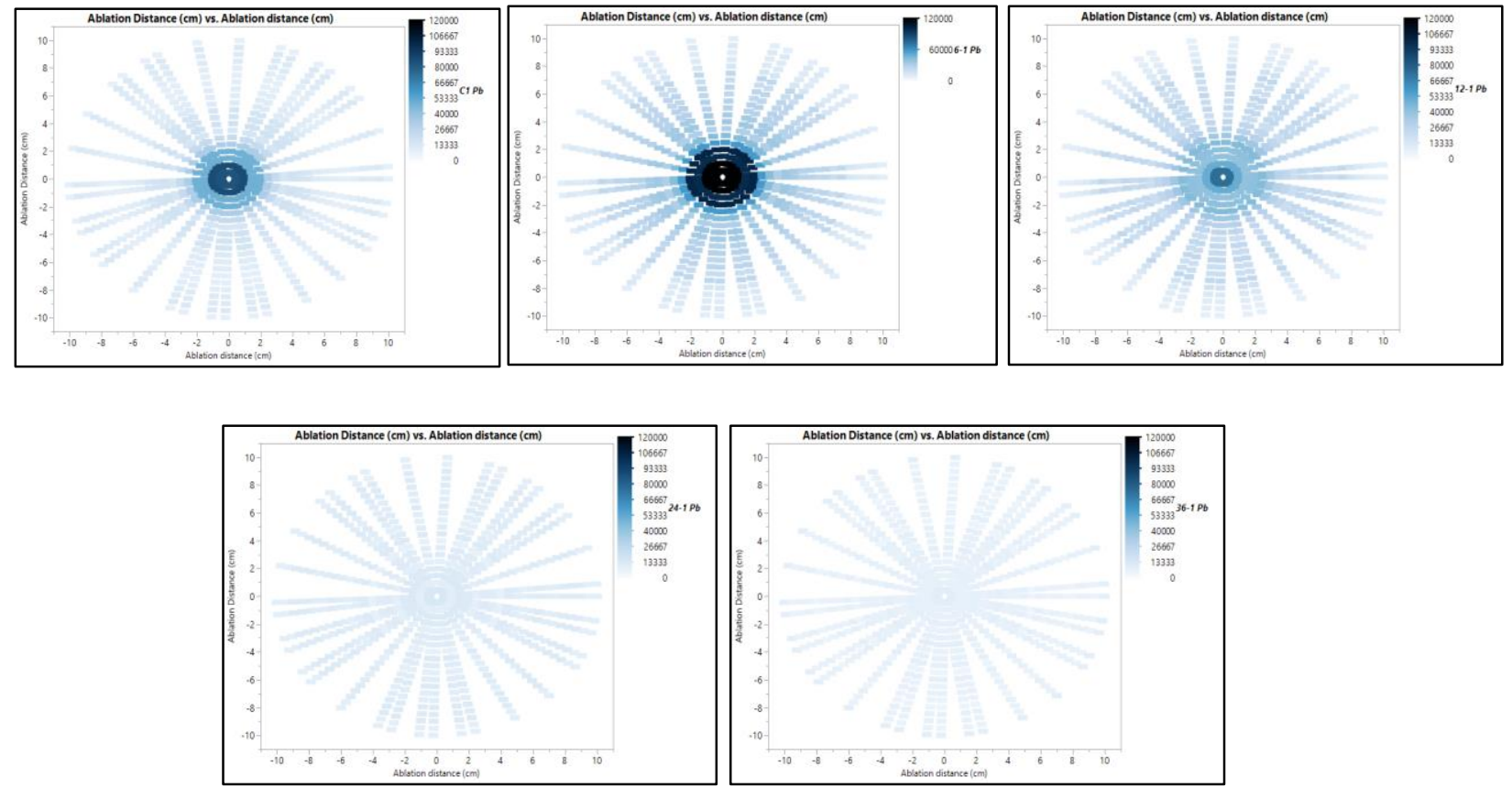

Figure 68. Heat maps of $\mathrm{Pb}(405.8 \mathrm{~nm})$ obtained from patterned calibration set number 1; From Left to Right: contact, 6 inches, 12 inches, 24 inches, 36 inches. 

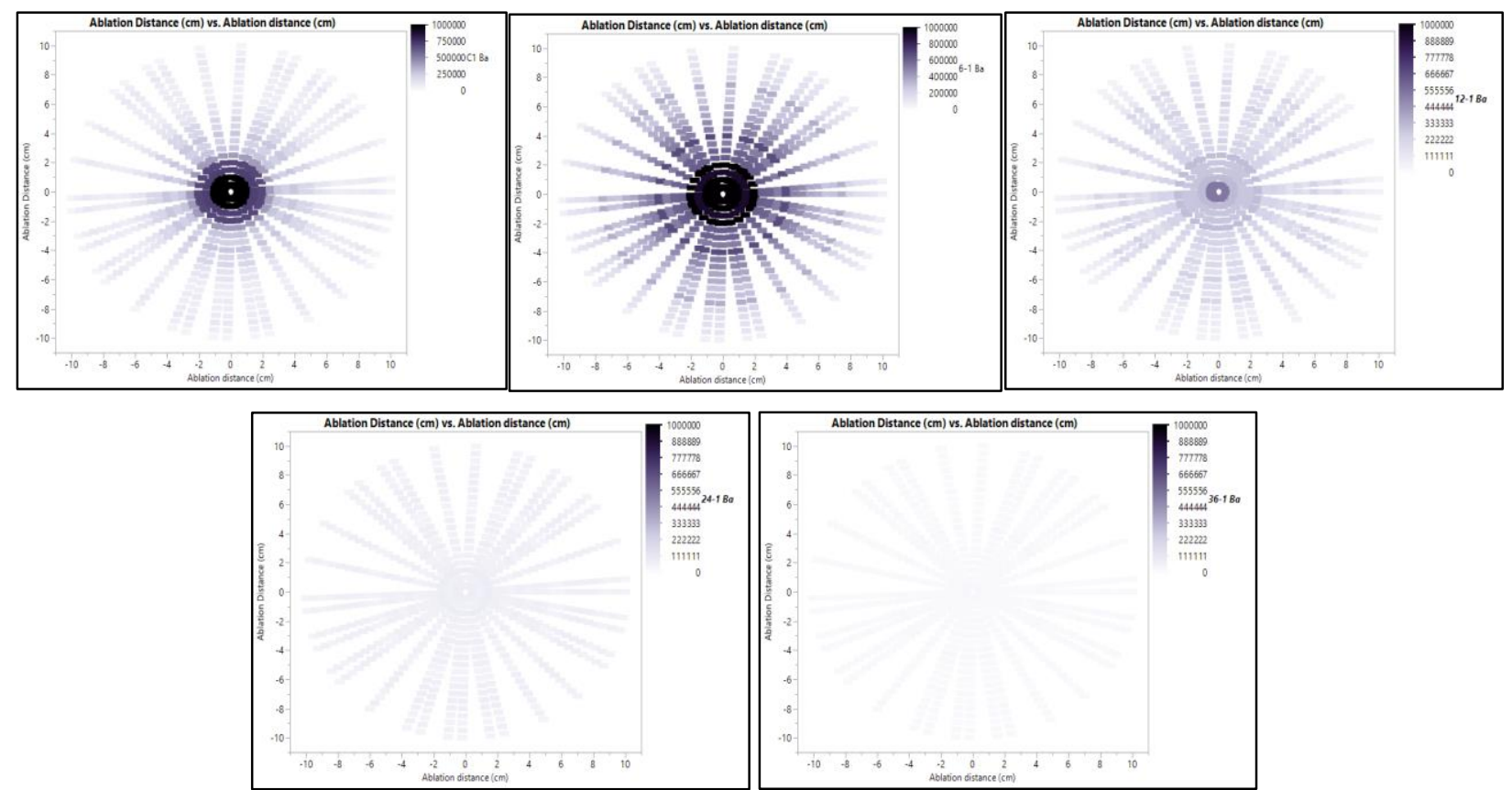

Figure 69. Heat maps of $\mathrm{Ba}(403.4 \mathrm{~nm})$ obtained from patterned calibration set number 1; From Left to Right: contact, 6 inches, 12 inches, 24 inches, 36 inches.
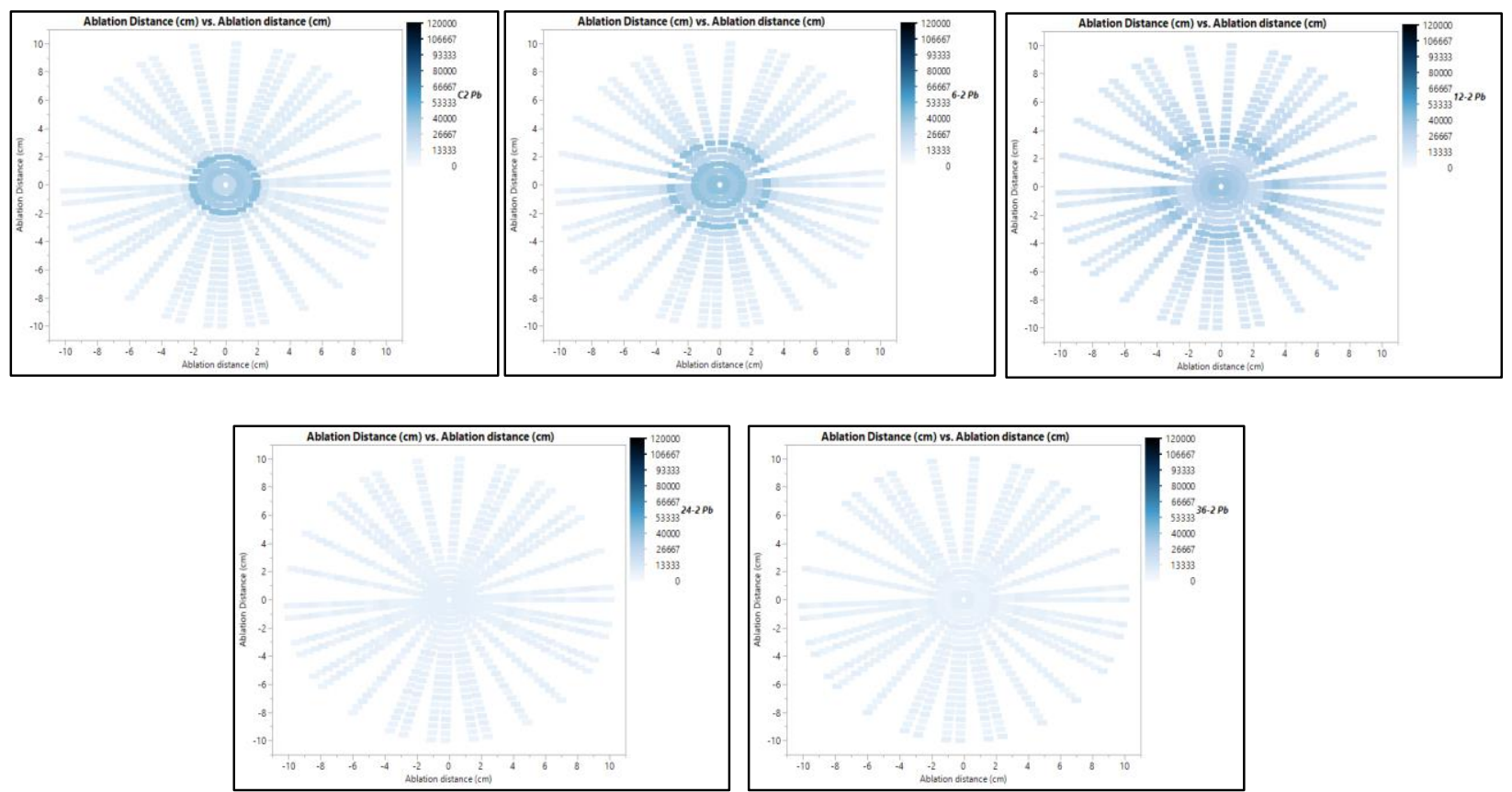

Figure 70. Heat maps of $\mathrm{Pb}(405.8 \mathrm{~nm})$ obtained from patterned calibration set number 2; From Left to Right: contact, 6 inches, 12 inches, 24 inches, 36 inches. 

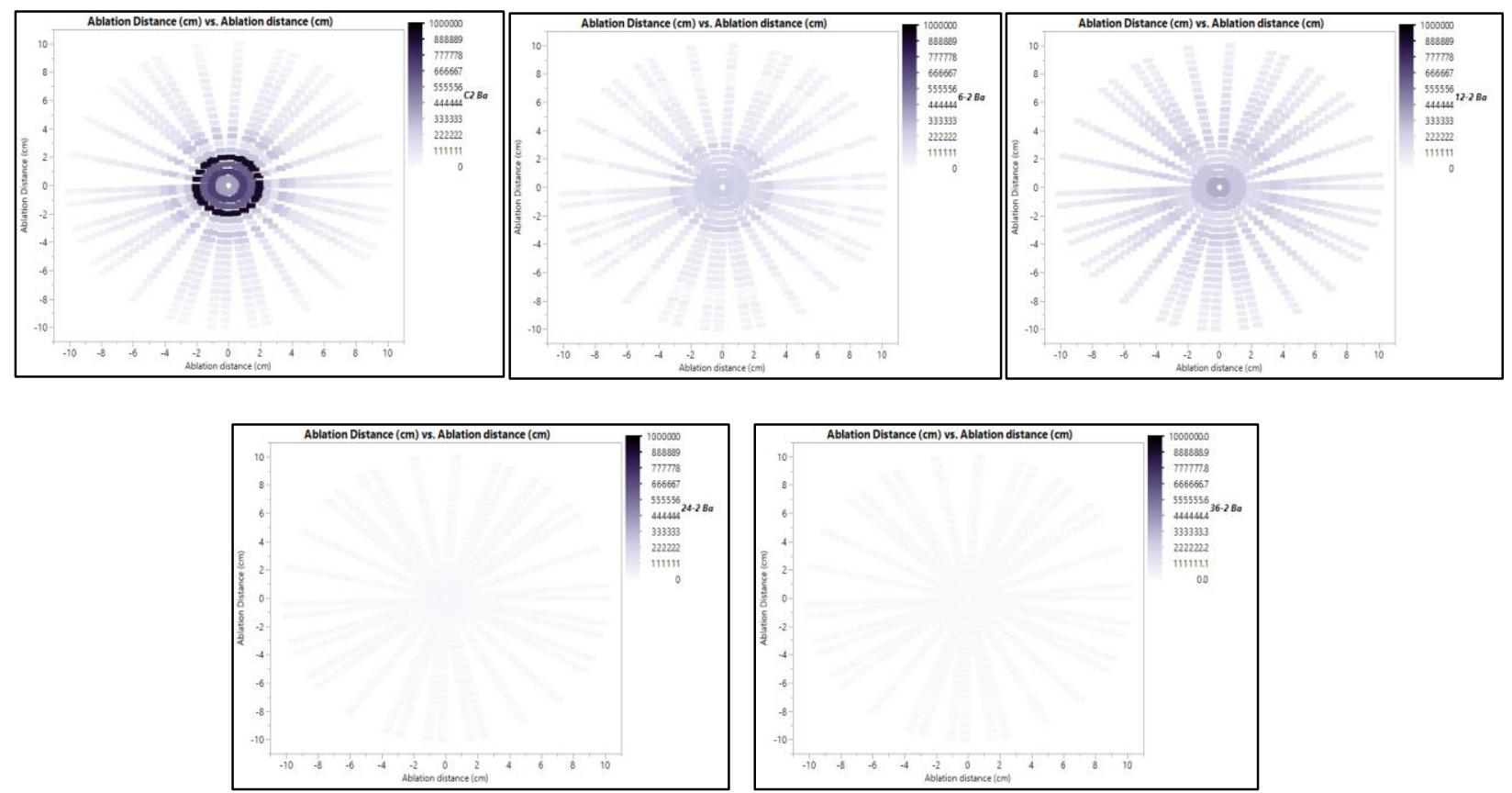

Figure 71. Heat maps of $\mathrm{Ba}(403.4 \mathrm{~nm})$ obtained from patterned calibration set number 2; From Left to Right: contact, 6 inches, 12 inches, 24 inches, 36 inches.
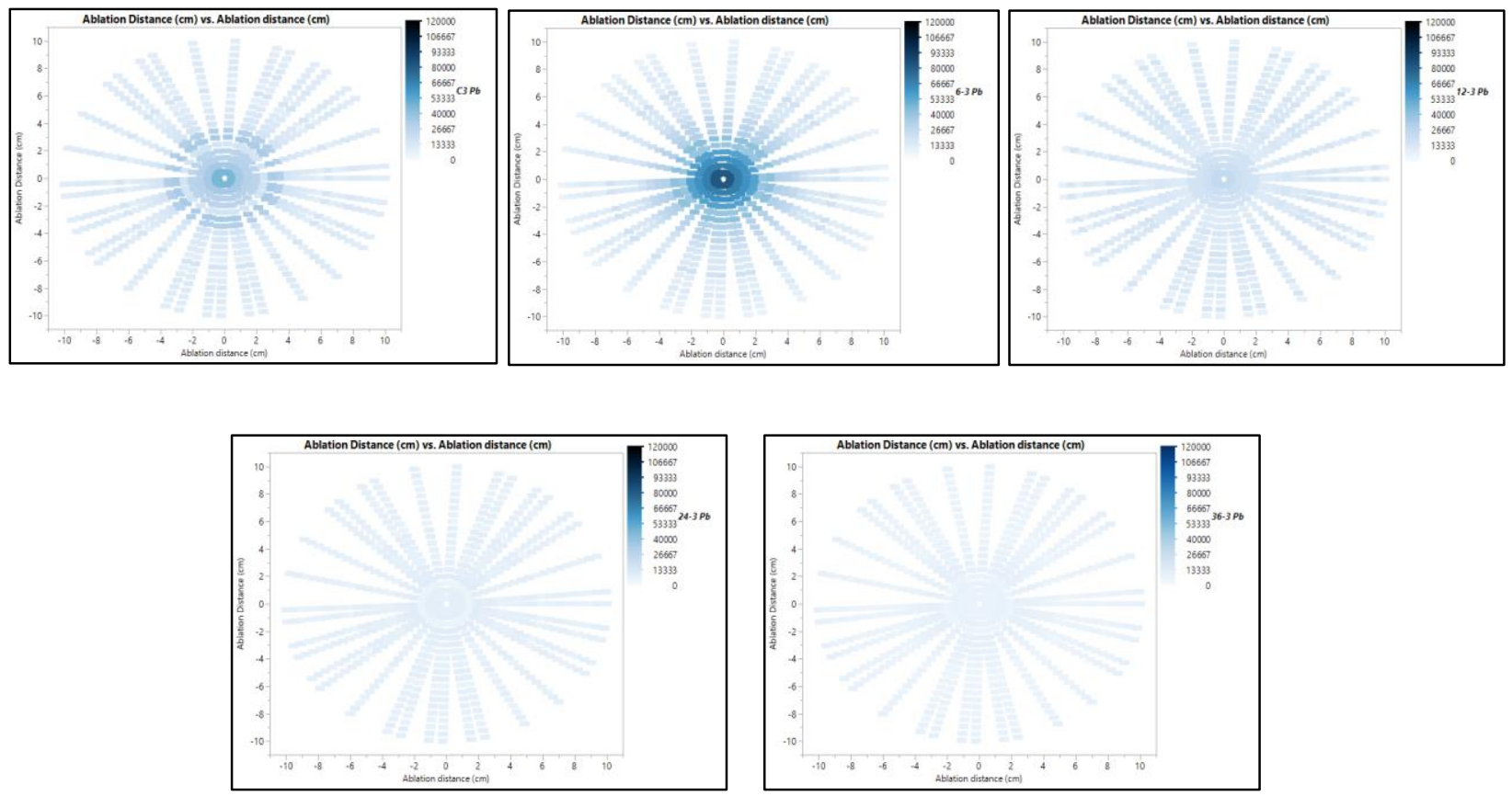

Figure 72. Heat maps of $\mathrm{Pb}(405.8 \mathrm{~nm})$ obtained from patterned calibration set number 3; From Left to Right: contact, 6 inches, 12 inches, 24 inches, 36 inches. 

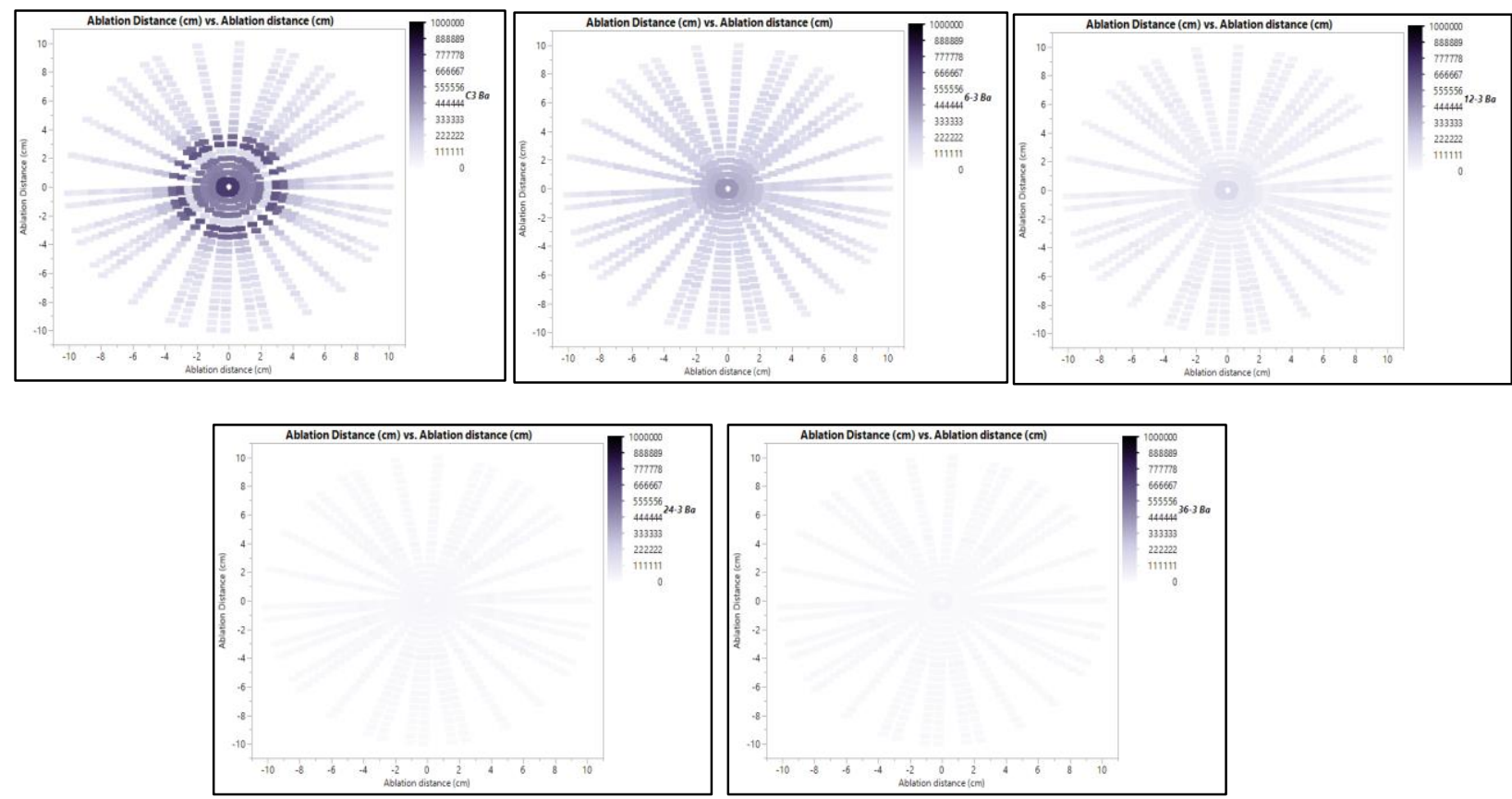

Figure 73. Heat maps of $\mathrm{Ba}(403.4 \mathrm{~nm})$ obtained from patterned calibration set number 3; From Left to Right: contact, 6 inches, 12 inches, 24 inches, 36 inches.
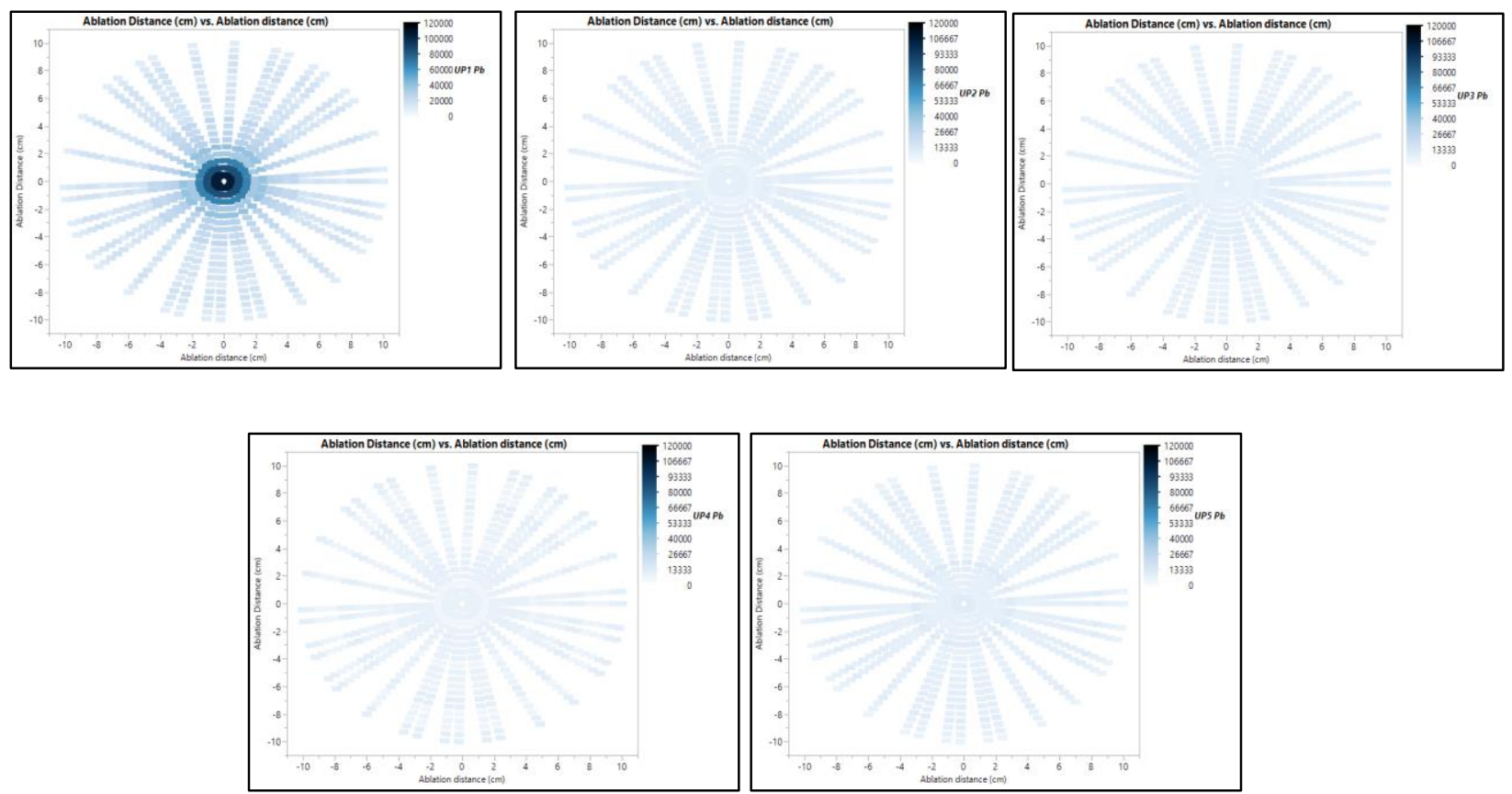

Figure 74. Heat maps of $\mathrm{Pb}(405.8 \mathrm{~nm})$ obtained from patterned unknowns; From Left to Right: patterned unknown 1, patterned unknown 2, patterned unknown 3, patterned unknown 4, patterned unknown 5. 

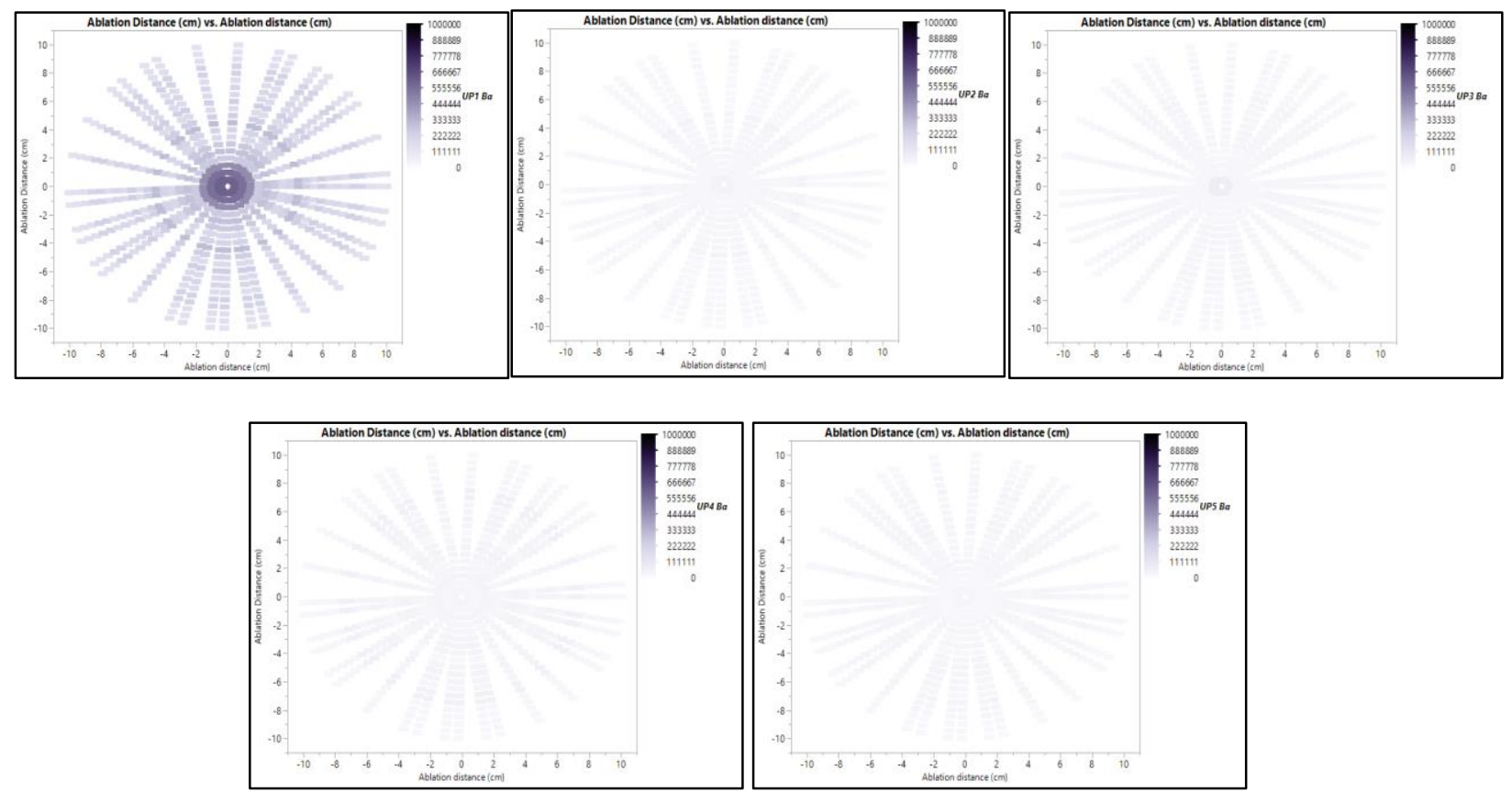

Figure 75. Heat maps of Ba (493.4 nm) obtained from patterned unknowns; From Left to Right: patterned unknown 1, patterned unknown 2, patterned unknown 3, patterned unknown 4, patterned unknown 5.
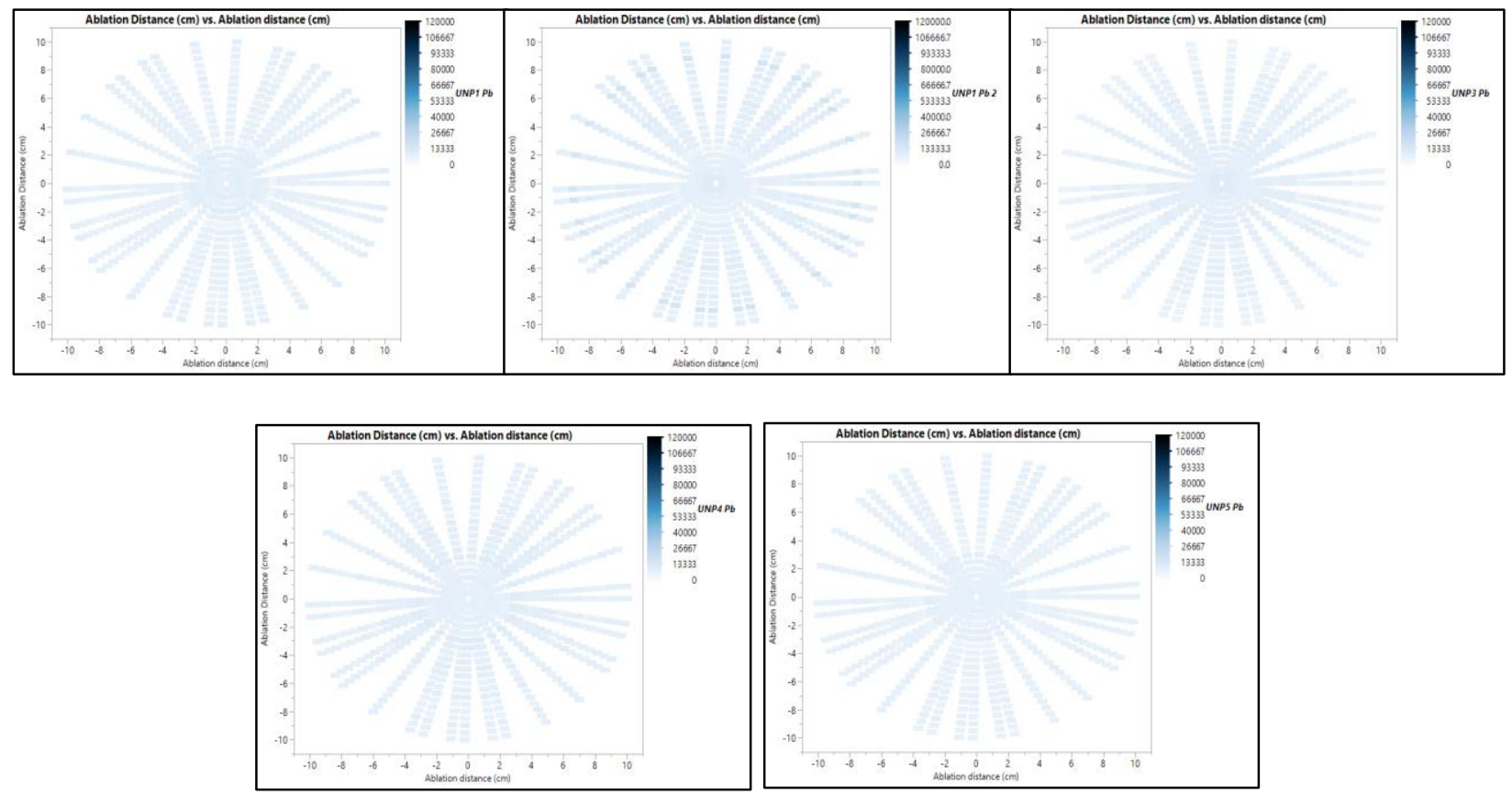

Figure 76. Heat maps of $\mathrm{Pb}(405.8 \mathrm{~nm})$ obtained from non-patterned unknowns; From Left to Right: non-patterned unknown 1, non- patterned unknown 2, non-patterned unknown 3, non-patterned unknown 4, non-patterned unknown 5. 

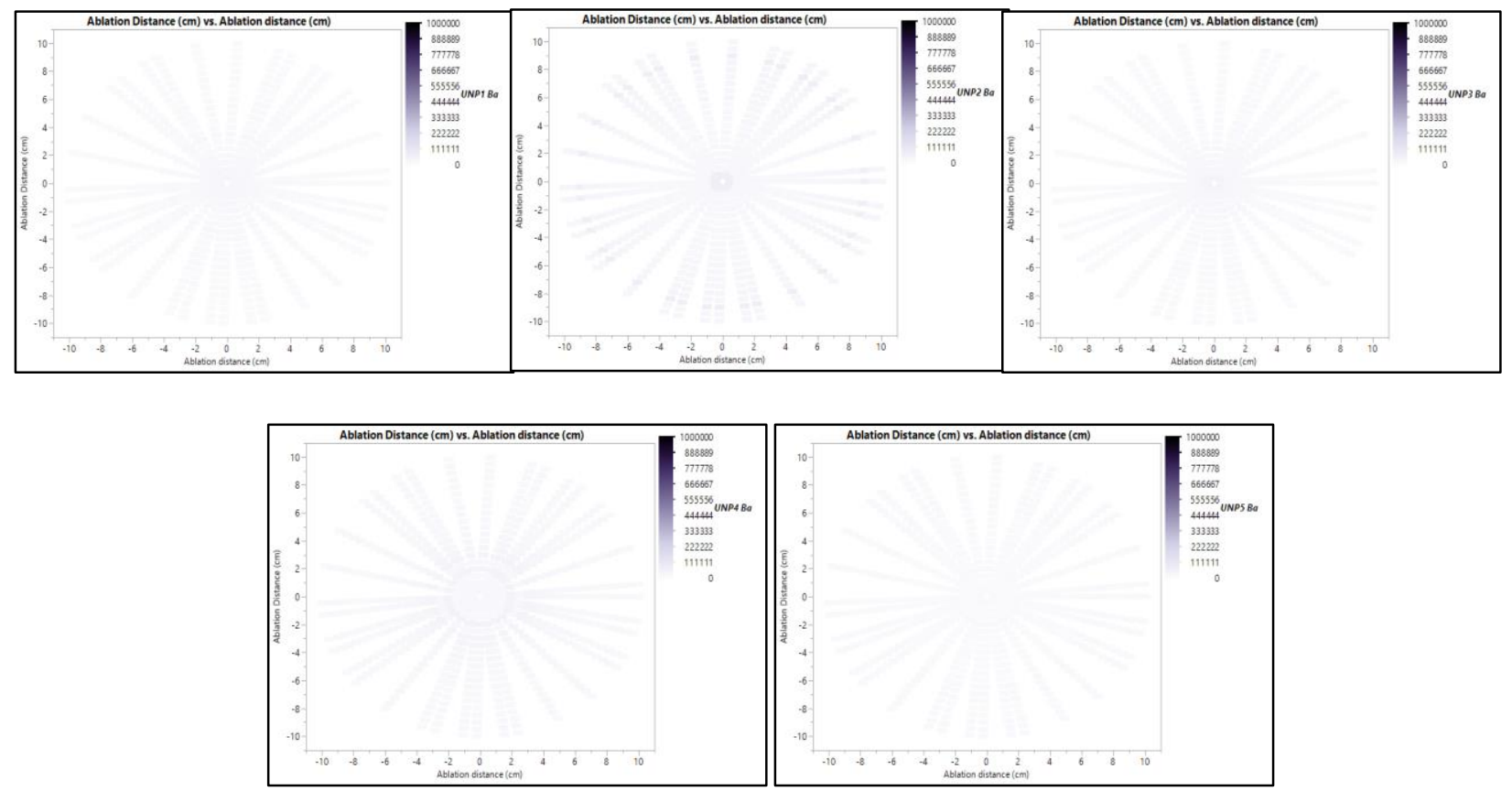

Figure 77. Heat maps of $\mathrm{Ba}(493.4 \mathrm{~nm})$ obtained from non-patterned unknowns; From Left to Right: non-patterned unknown 1, non- patterned unknown 2, non-patterned unknown 3, non-patterned unknown 4, non-patterned unknown 5.
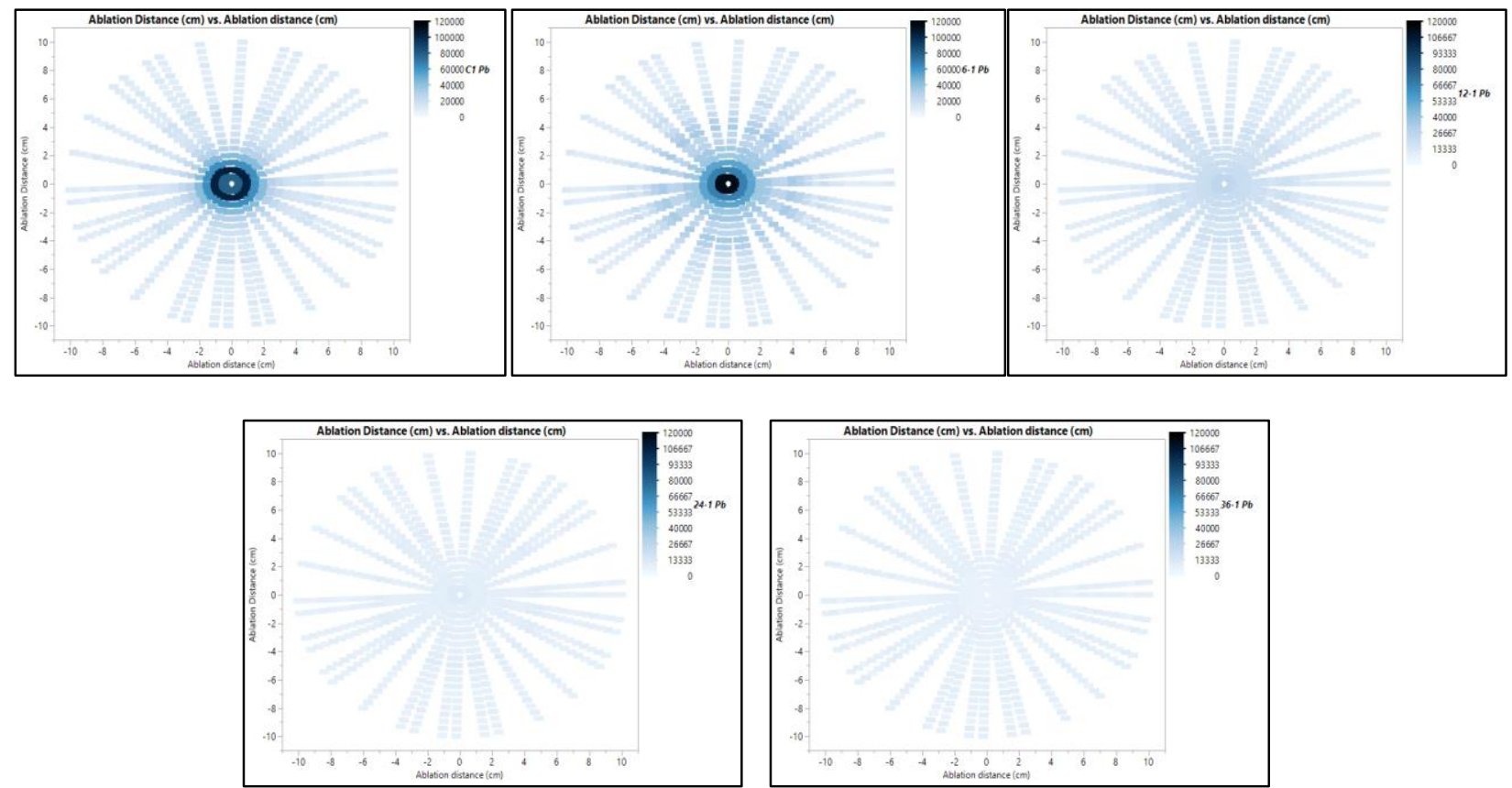

Figure 78. Heat maps of $\mathrm{Pb}(405.8 \mathrm{~nm})$ obtained from blood calibration set number 1; From Left to Right: contact, 6 inches, 12 inches, 24 inches, 36 inches. 

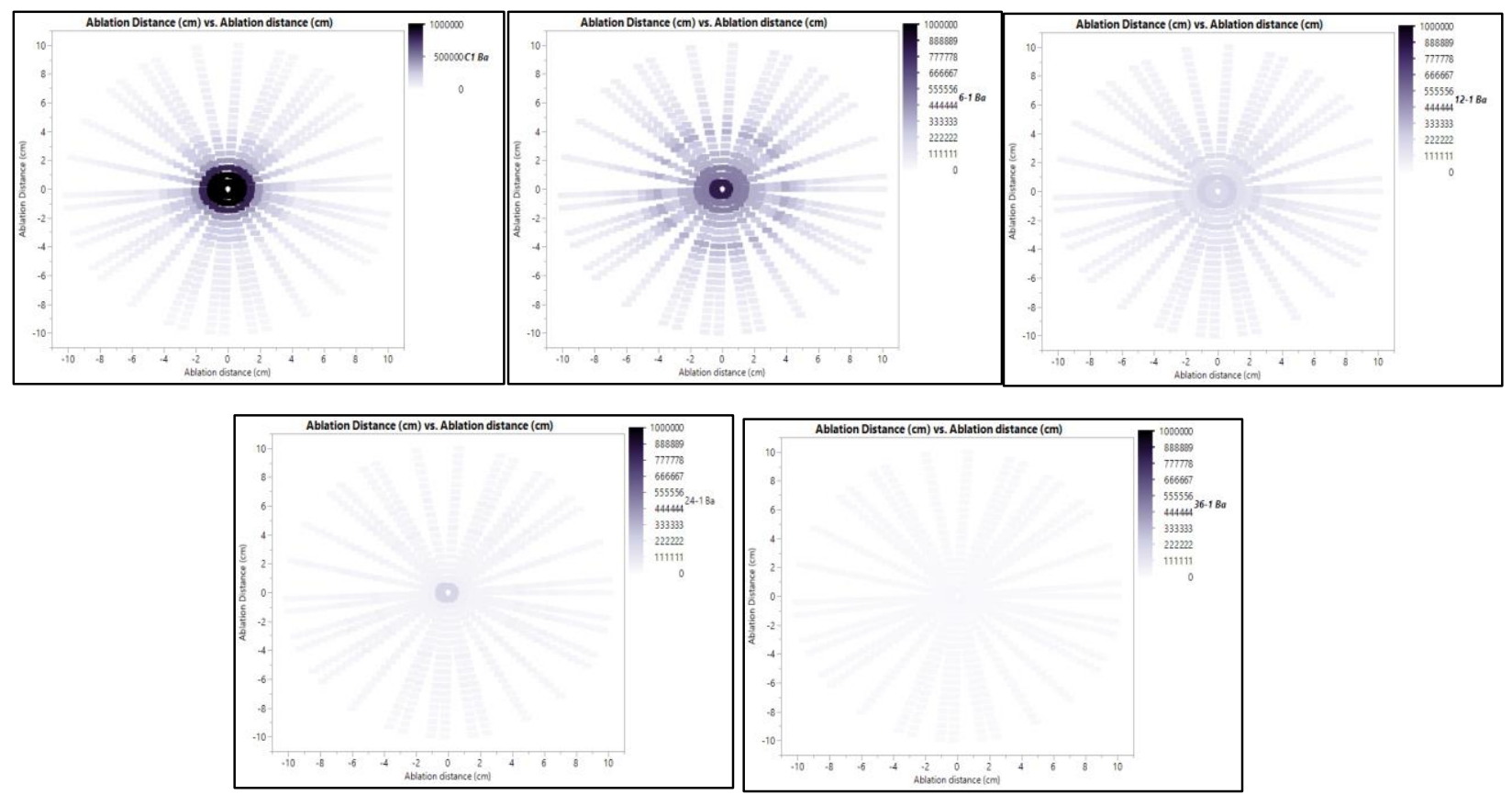

Figure 79. Heat maps of $\mathrm{Ba}(493.4 \mathrm{~nm})$ obtained from blood calibration set number 1; From Left to Right: contact, 6 inches, 12 inches, 24 inches, 36 inches.
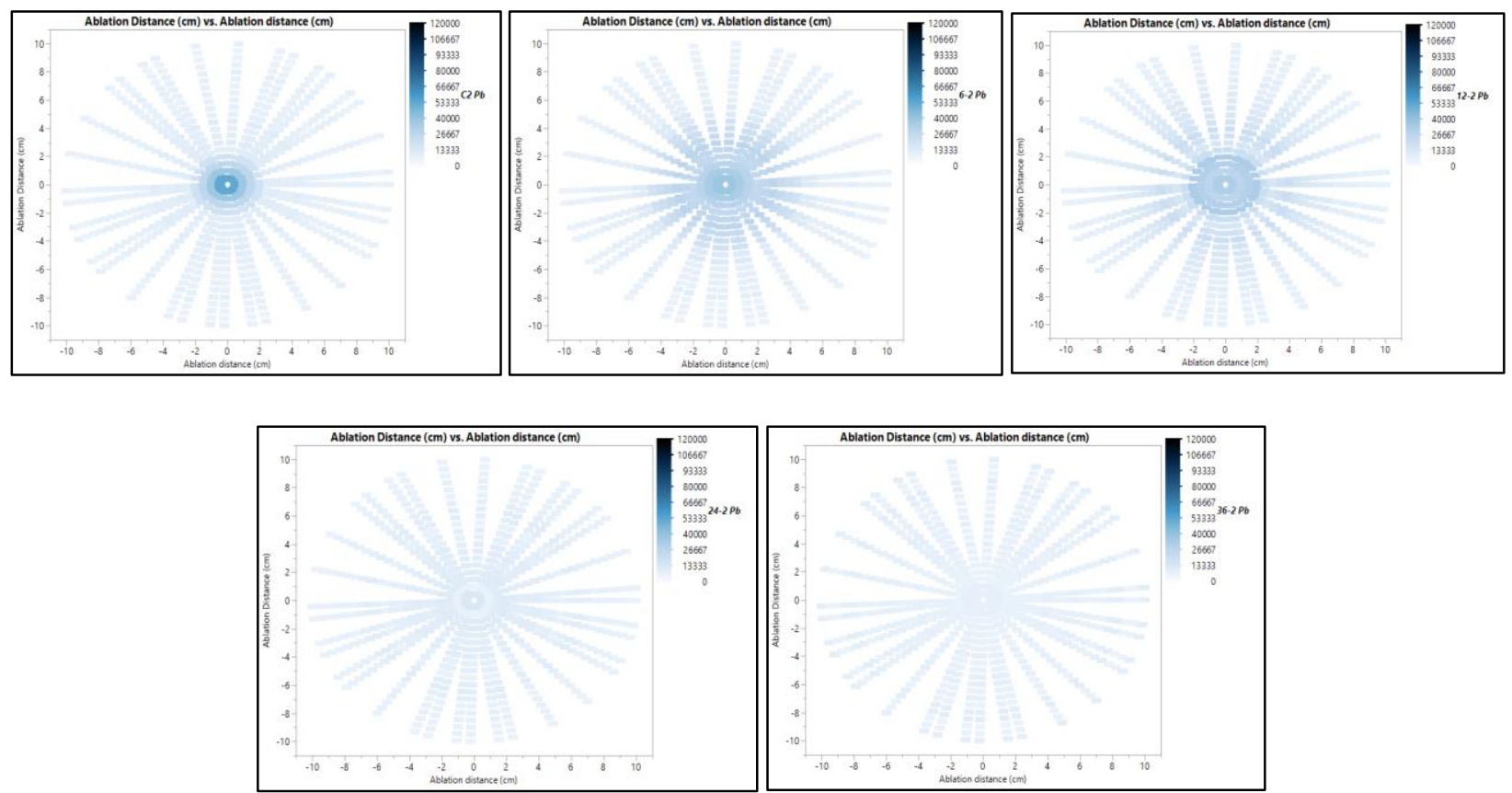

Figure 80. Heat maps of $\mathrm{Pb}(405.8 \mathrm{~nm})$ obtained from blood calibration set number 2; From Left to Right: contact, 6 inches, 12 inches, 24 inches, 36 inches. 

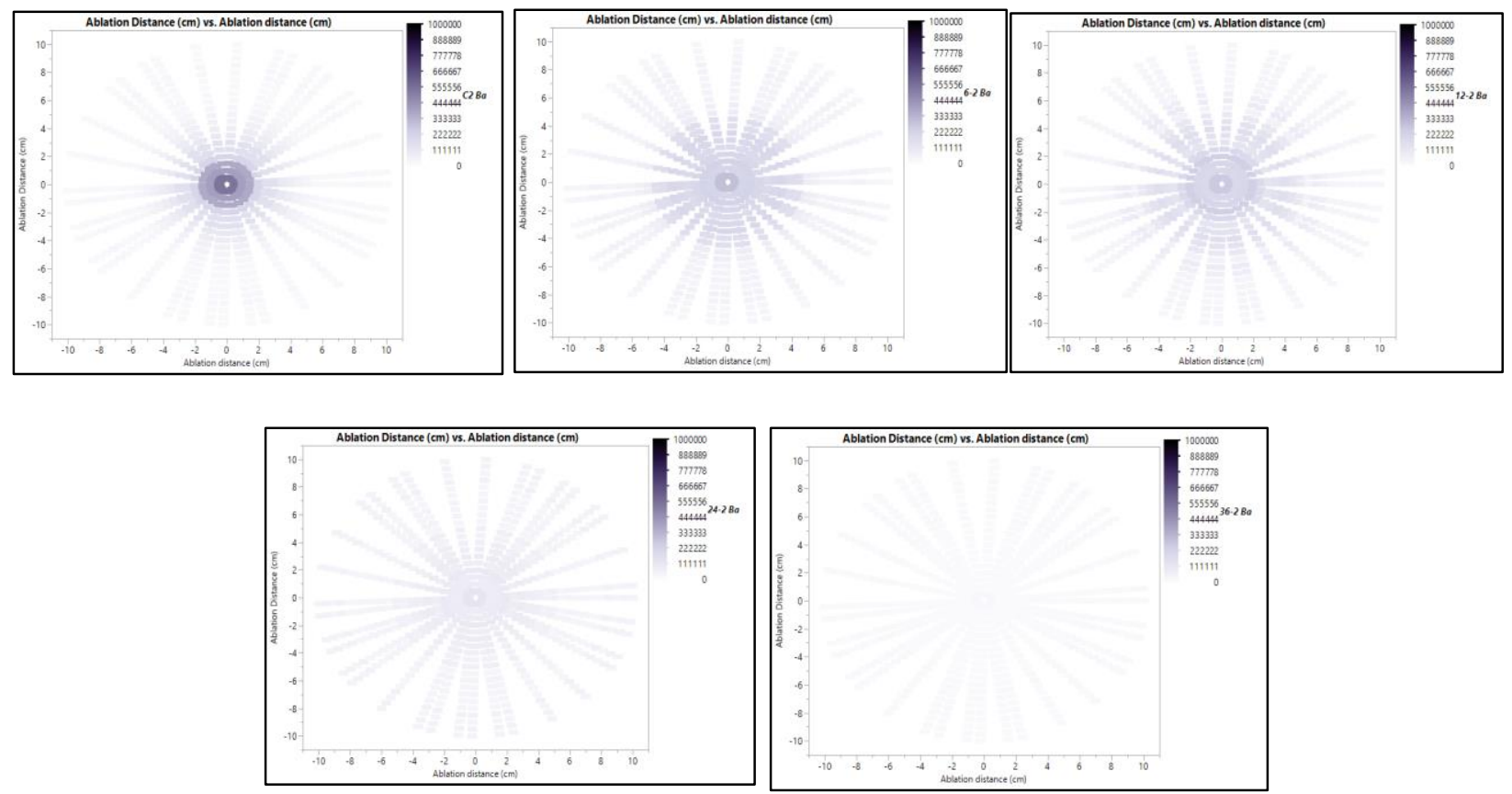

Figure 81. Heat maps of Ba (493.4 nm) obtained from blood calibration set number 2; From Left to Right: contact, 6 inches, 12 inches, 24 inches, 36 inches.
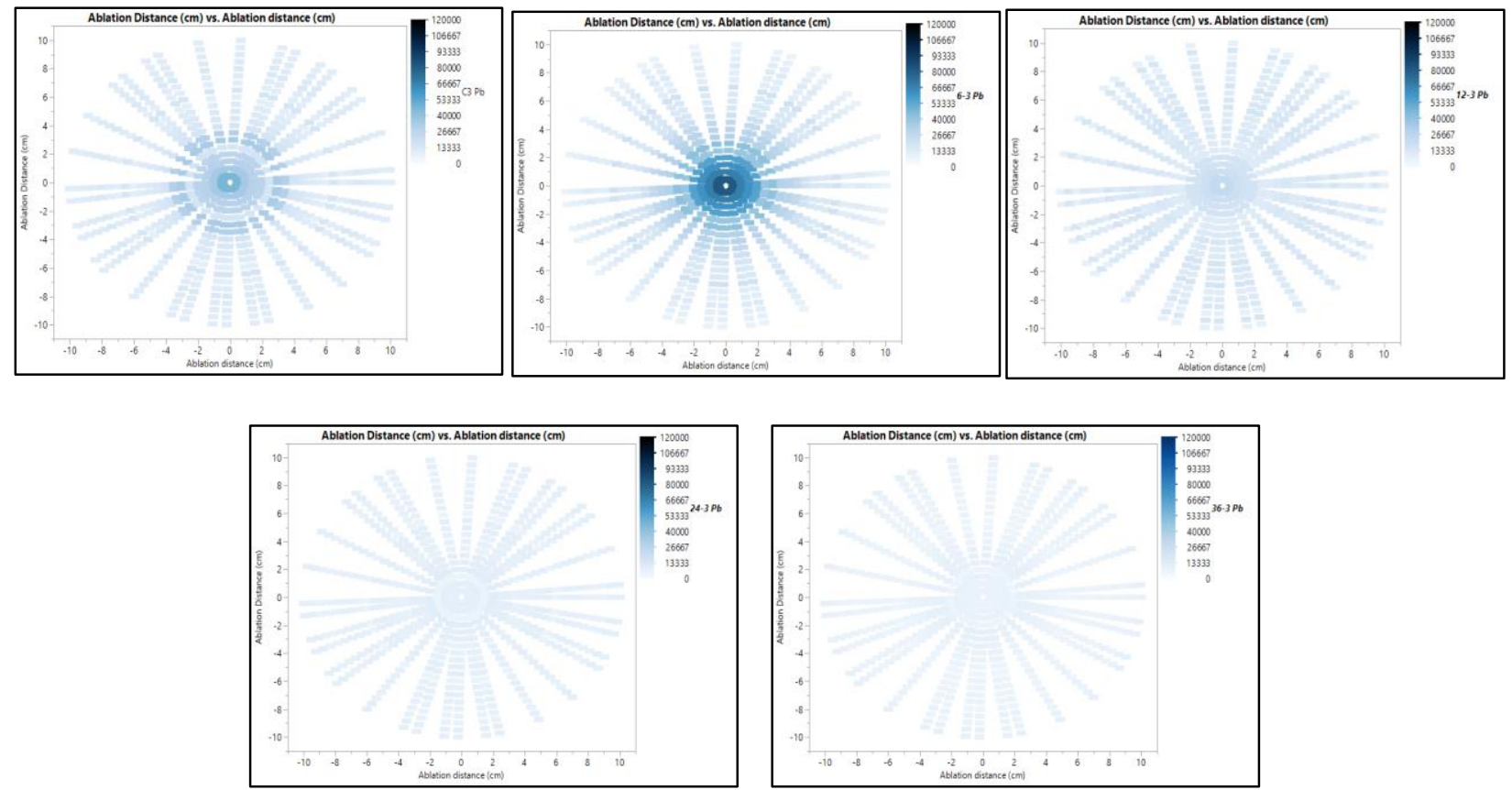

Figure 82. Heat maps of $\mathrm{Pb}(405.8 \mathrm{~nm})$ obtained from blood calibration set number 3; From Left to Right: contact, 6 inches, 12 inches, 24 inches, 36 inches. 

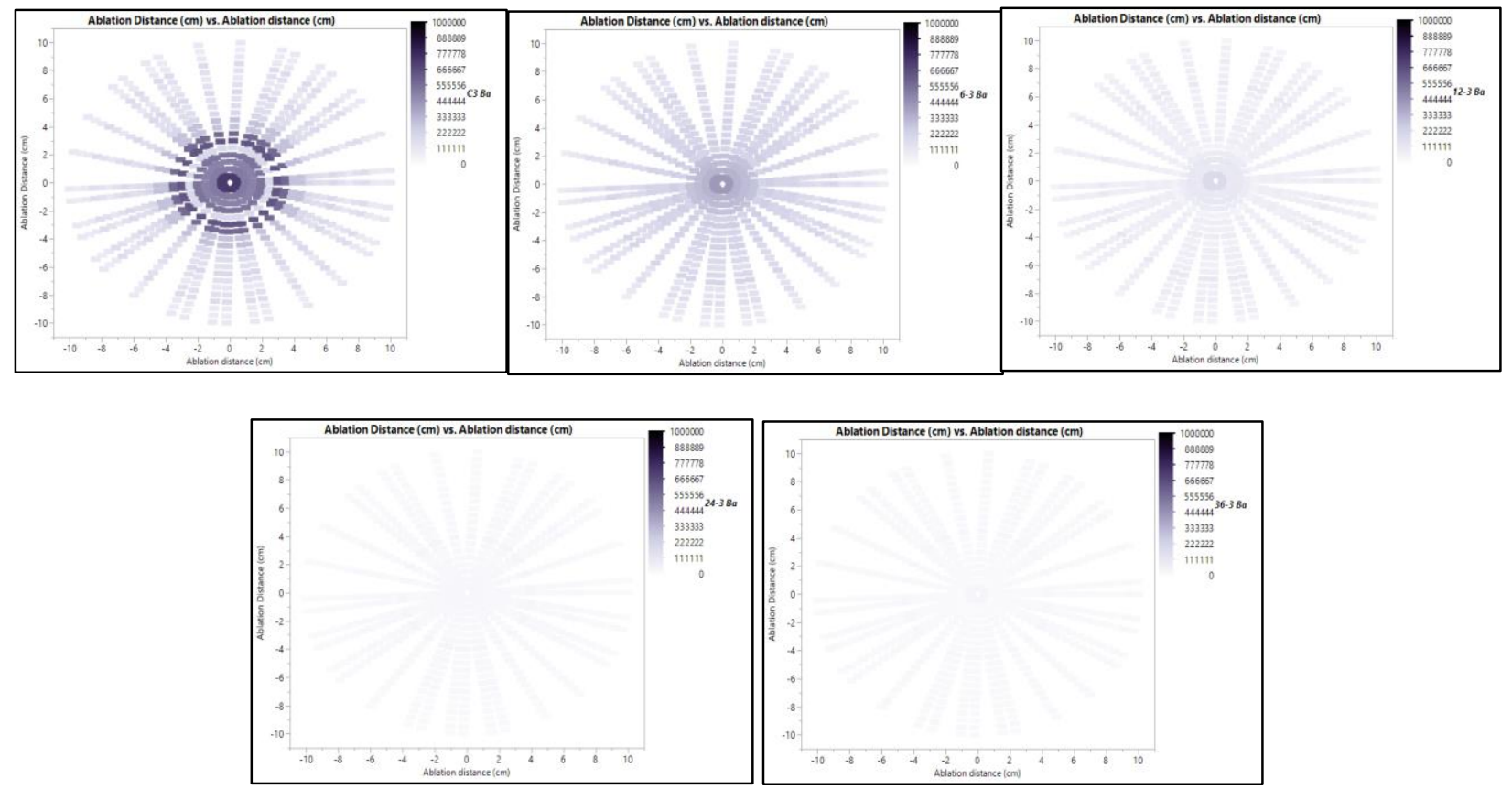

Figure 83. Heat maps of $\mathrm{Ba}(493.4 \mathrm{~nm})$ obtained from blood calibration set number 3 ; From Left to Right: contact, 6 inches, 12 inches, 24 inches, 36 inches.
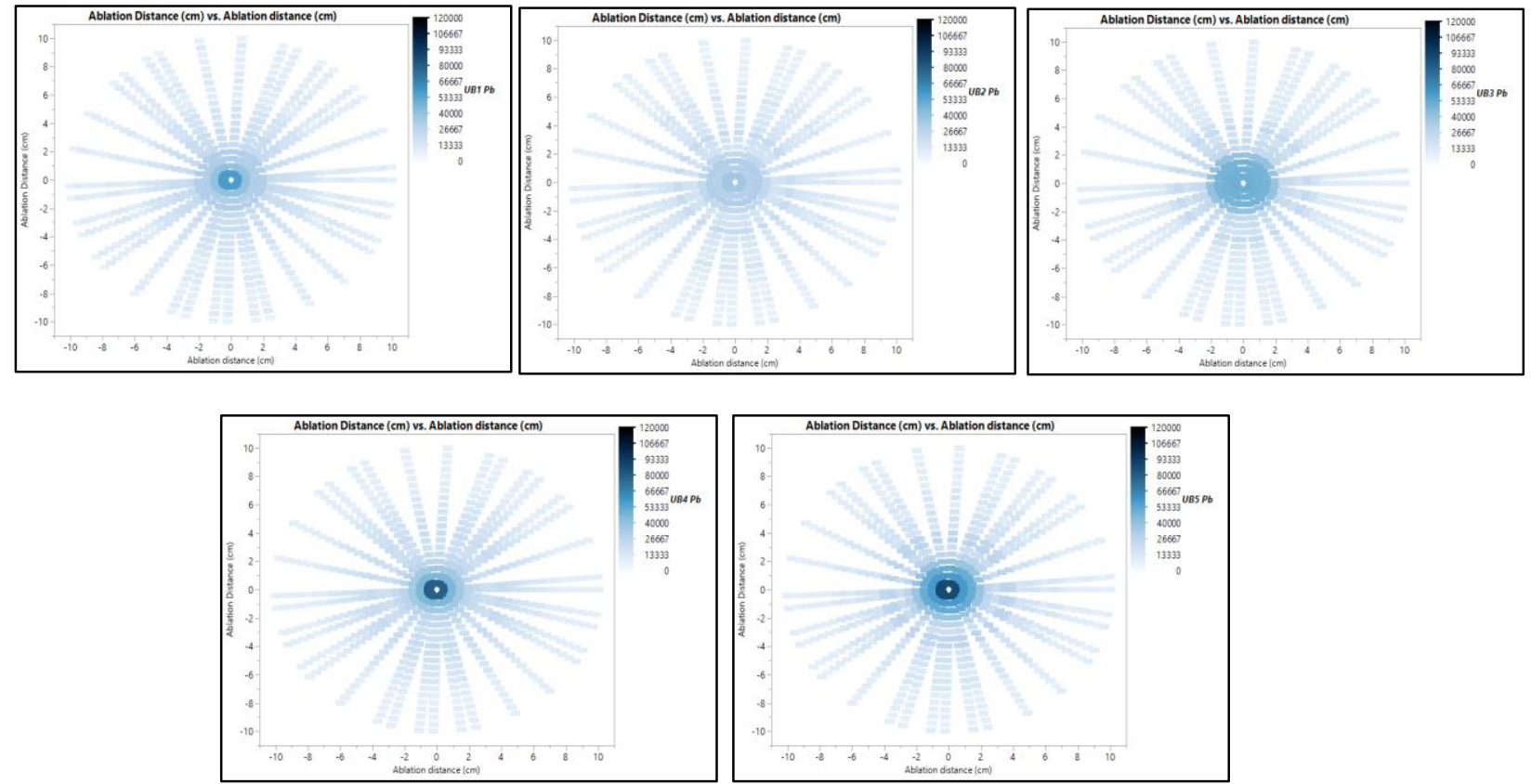

Figure 84. Heat maps of $\mathrm{Pb}(405.8 \mathrm{~nm})$ obtained from blood unknowns; From Left to Right: blood unknown 1, blood unknown 2, blood unknown 3, blood unknown 4, blood unknown 5 . 

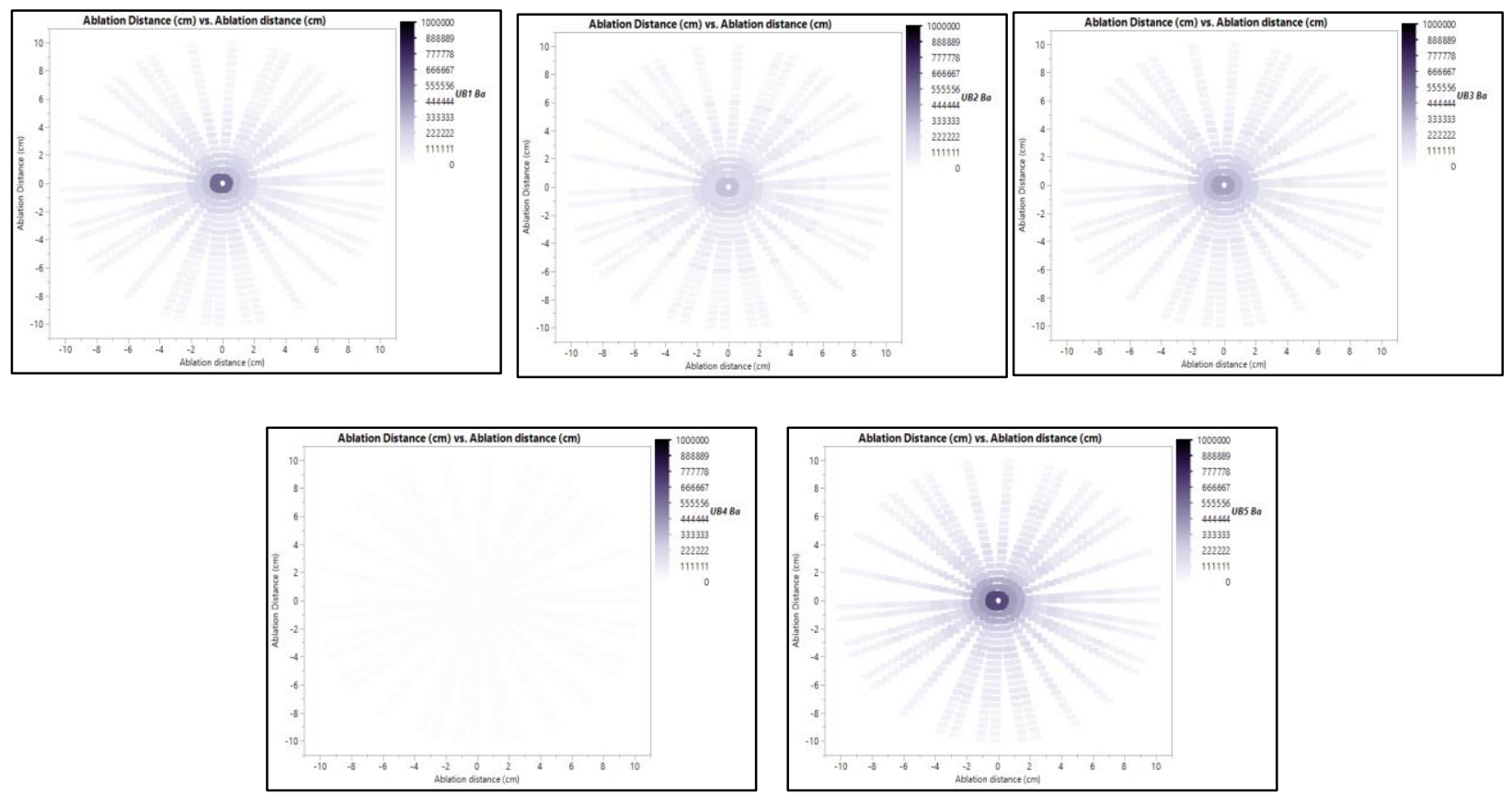

Figure 85. Heat maps of Ba (493.4 nm) obtained from blood unknowns; From Left to Right: blood unknown 1, blood unknown 2, blood unknown 3, blood unknown 4, blood unknown 5 .
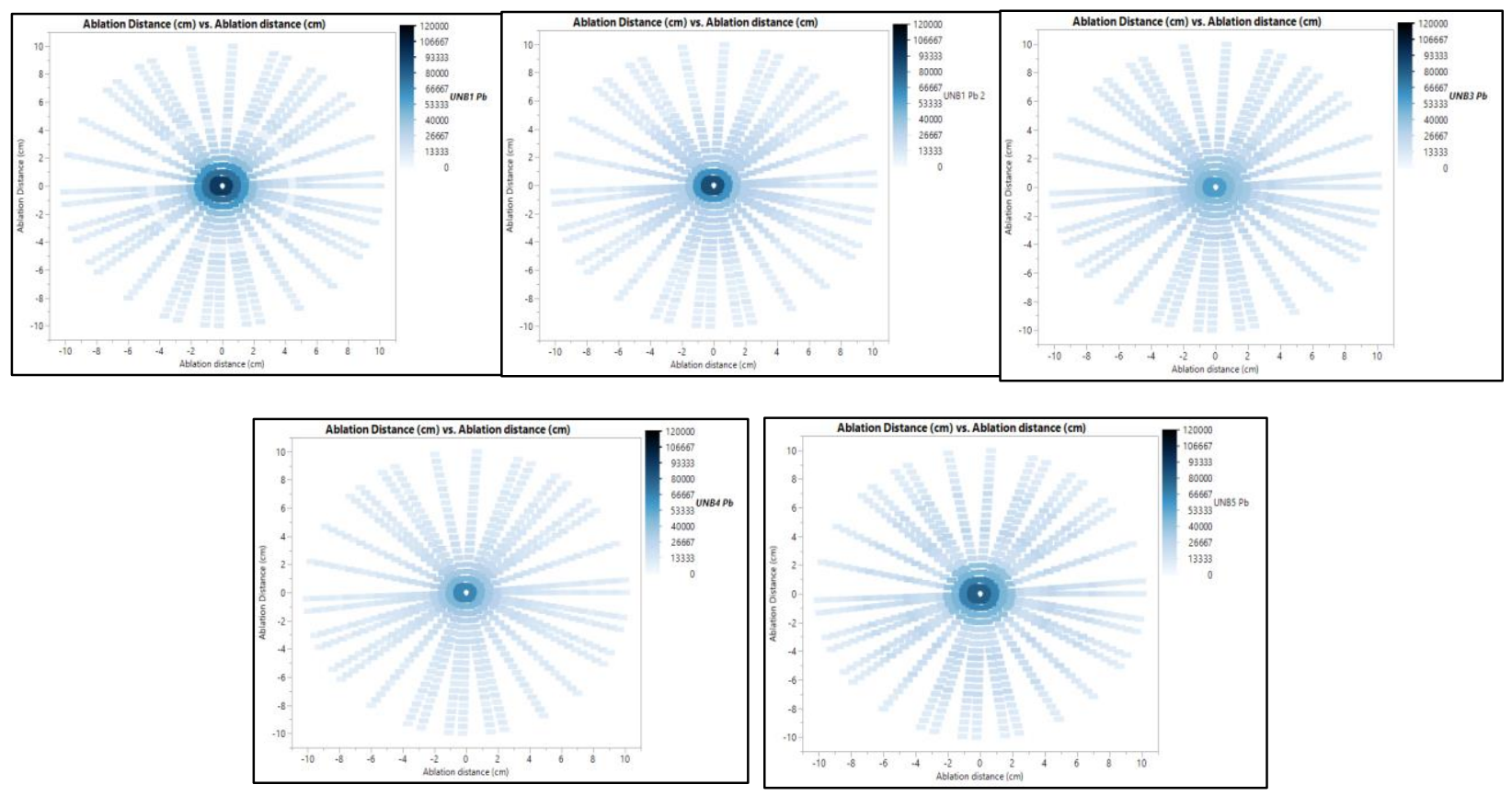

Figure 86. Heat maps of $\mathrm{Pb}(405.8 \mathrm{~nm})$ obtained from non-blood unknowns; From Left to Right: non-blood unknown 1, non-blood unknown 2, non-blood unknown 3, non-blood unknown 4, non-blood unknown 5. 

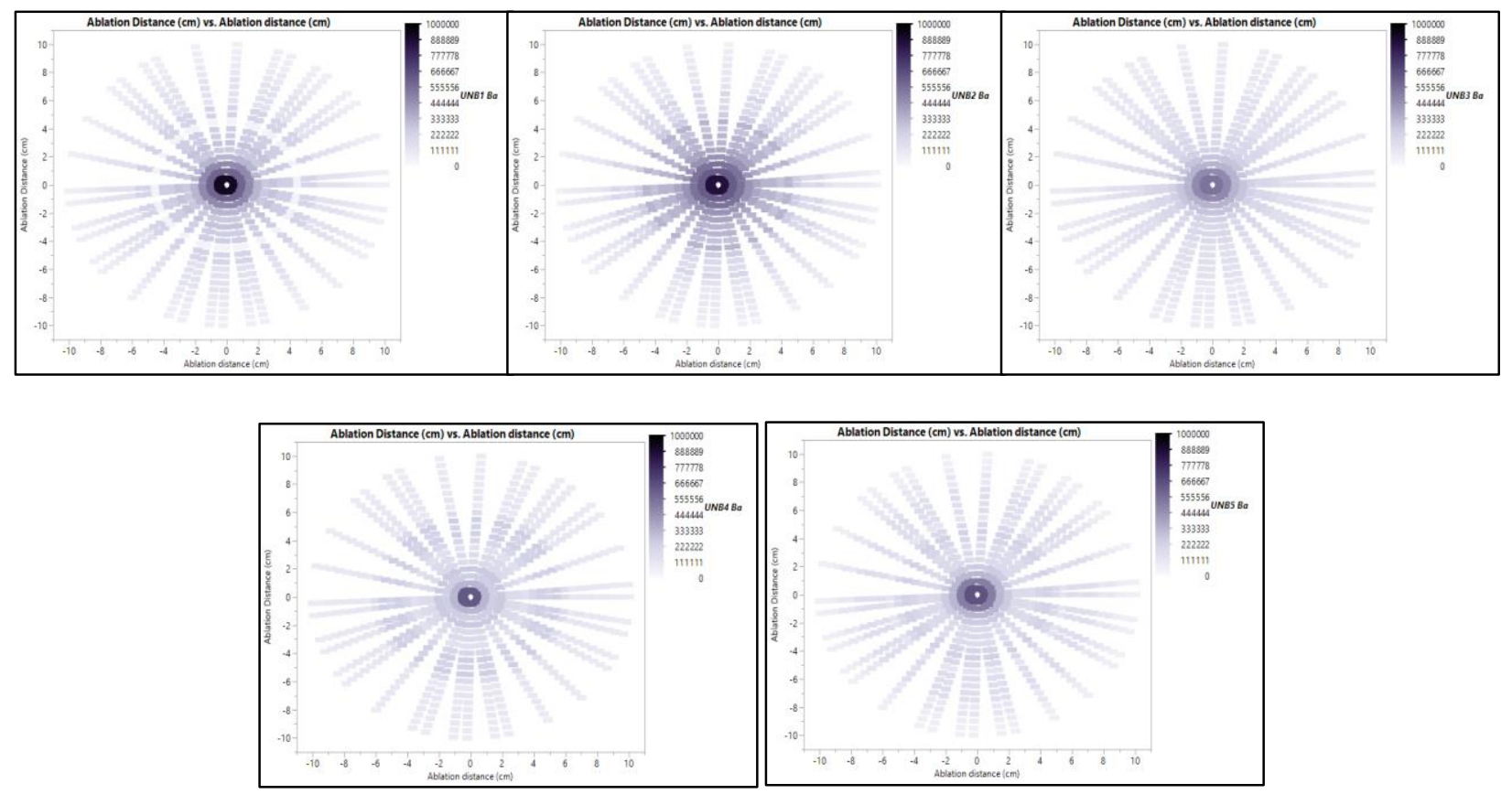

Figure 87. Heat maps of $\mathrm{Pb} \mathrm{Ba}(493.4 \mathrm{~nm})$ obtained from non-blood unknowns; From Left to Right: non-blood unknown 1, non-blood unknown 2, non-blood unknown 3, non-blood unknown 4, non-blood unknown 5.
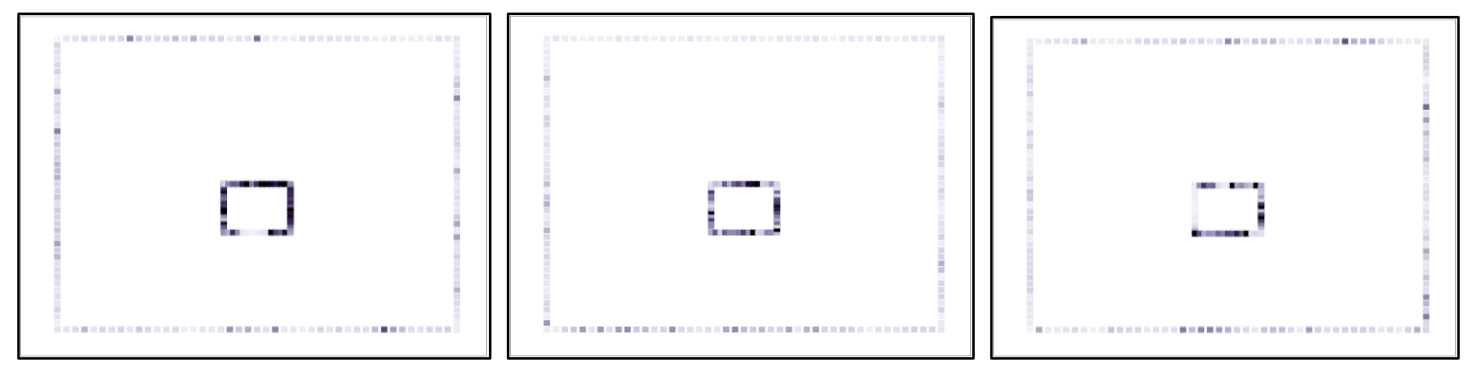

Figure 88. Heat maps of $\mathrm{Pb}(405.8 \mathrm{~nm})$ for bullet hole identification, obtained from wood substrates; From Left to Right: Wood substrate 2, Wood substrate 3, Wood substrate 4.
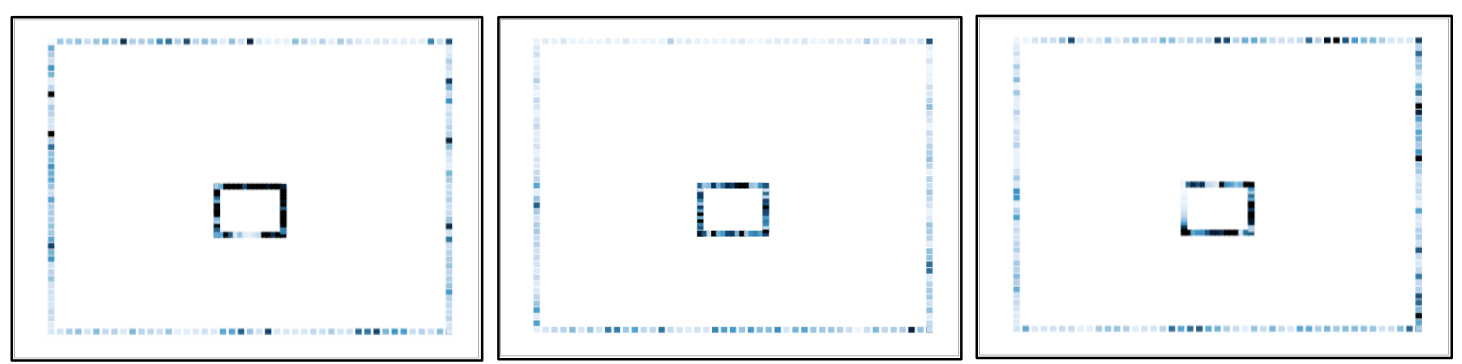

Figure 89. Heat maps of $\mathrm{Ba}(493.4 \mathrm{~nm})$ for bullet hole identification, obtained from wood substrates; From Left to Right: Wood substrate 2, Wood substrate 3, Wood substrate 4. 

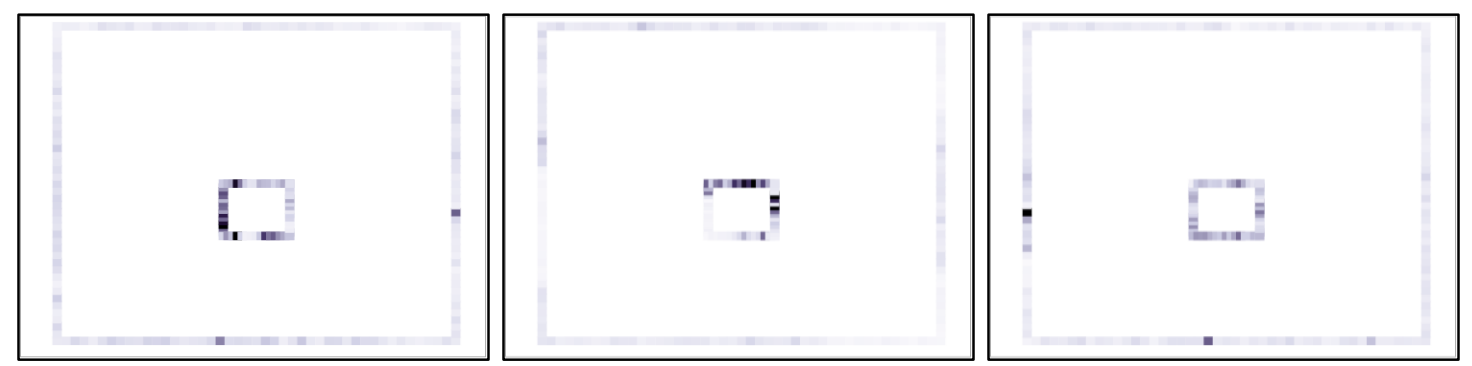

Figure 90. Heat maps of $\mathrm{Pb}(405.8 \mathrm{~nm})$ for bullet hole identification, obtained from drywall substrates; From Left to Right: Drywall substrate 2, Drywall substrate 3, Drywall substrate 4.
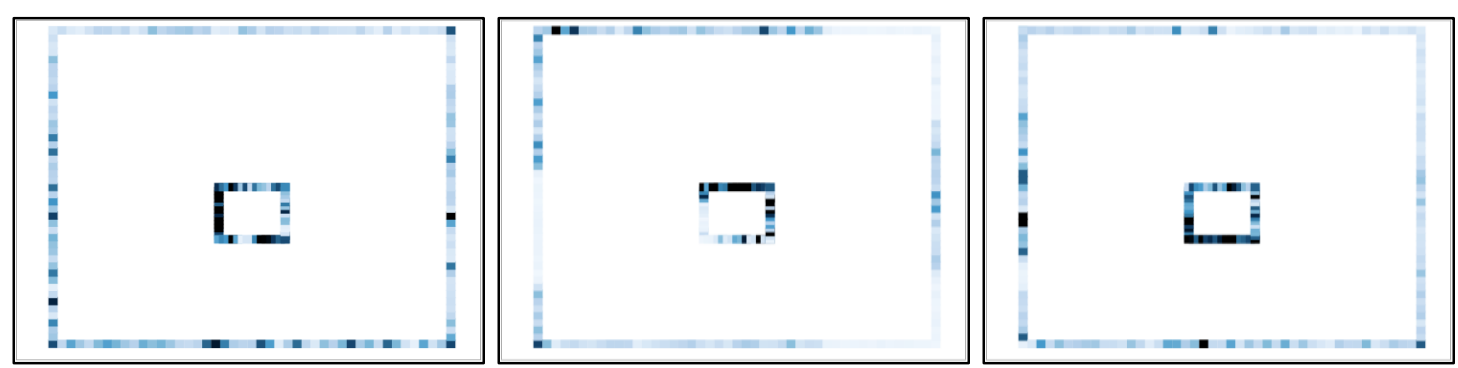

Figure 91. Heat maps of $\mathrm{Ba}(493.4 \mathrm{~nm})$ for bullet hole identification, obtained from drywall substrates; From Left to Right: Drywall substrate 2, Drywall substrate 3, Drywall substrate 4.
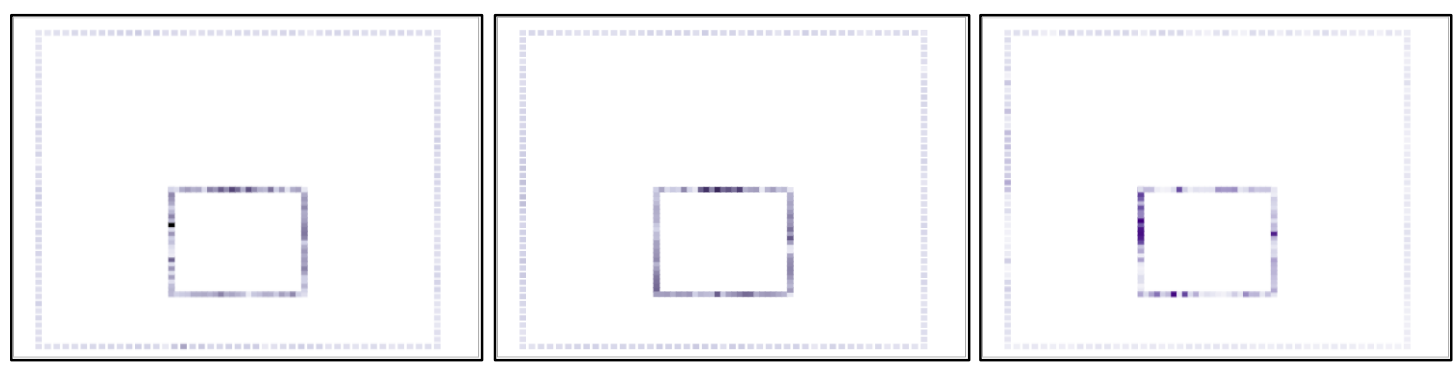

Figure 92. Heat maps of $\mathrm{Pb}(405.8 \mathrm{~nm})$ for bullet hole identification, obtained from glass substrates; From Left to Right: Glass substrate 2, Glass substrate 3, Glass substrate 4.

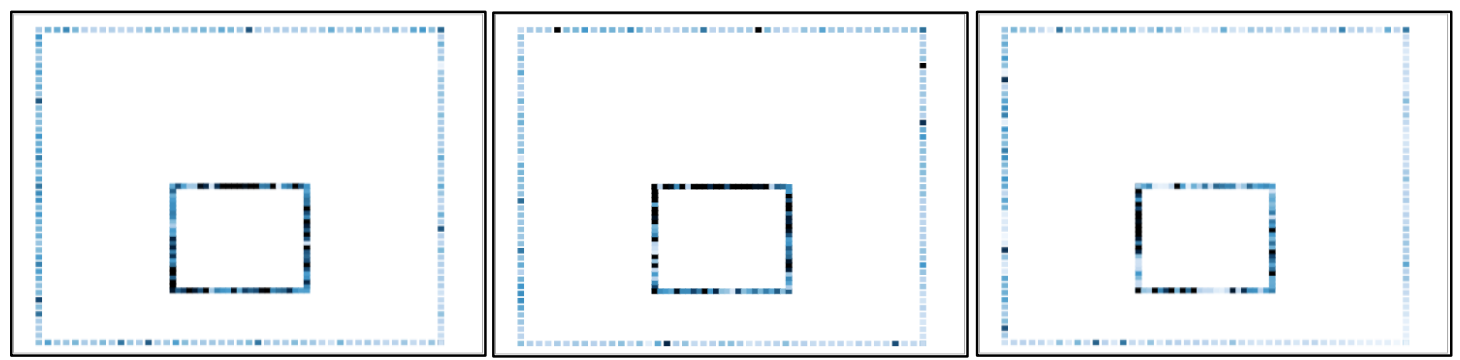

Figure 93. Heat maps of $\mathrm{Ba}(493.4 \mathrm{~nm})$ for bullet hole identification, obtained from glass substrates; From Left to Right: Glass substrate 2, Glass substrate 3, Glass substrate 4. 


\section{APPENDIX II. SPECTROCHEMICAL MAPPING USING LASER INDUCED BREAKDOWN SPECTROSCOPY AS A MORE OBJECTIVE APPROACH TO SHOOTING DISTANCE DETERMINATION}

The following appendix is the full, copyrighted version of the article referenced in Section 3.2. 


\title{
Spectrochemical mapping using laser induced breakdown spectroscopy as a more objective approach to shooting distance determination
}

\author{
Courtney Vander Pyl, Oriana Ovide, Mandy Ho, Bayram Yuksel, Tatiana Trejos* \\ West Virginia University, Department of Forensic and Investigative Science, 208 Oglebay Hall, Morgantown, WV 26506-6121, United States
}

\section{A R T I C L E I N F O}

\section{Keywords:}

Firearm discharge residues

Gunshot residues

Laser induced breakdown spectroscopy

Distance Determination

\begin{abstract}
A B S T R A C T
This study proposes the application of Laser-induced breakdown spectroscopy (LIBS) as a novel and practical method to detect gunshot residues around bullet holes and to estimate shooting distances in firearm-related criminal events. Clothing samples fired at different distance intervals were analyzed by LIBS, and the spectral data collected from these experiments were used to construct 2D elemental maps. Spatial distributions of inorganic gunshot residues (IGSR), such as lead, barium, and antimony, were used to classify the shooting distance range. Seventy-three cotton textiles of different color and fabric patterns were shot using a $9 \mathrm{~mm}$ pistol and a Rossi revolver with different ammunition. Forty-five of the fabrics were shot at known distances to create a control training set, while the remaining twenty-eight samples were used as blind items, of unknown distances to the examiner, to test the accuracy of the method. The performance of the LIBS method was compared to visual and colorimetric tests currently used by crime laboratories. Results show that LIBS offers superior sensitivity, selectivity, reproducibility, and accuracy compared to color tests. Unlike the conventional assays, LIBS produces permanent chemical images that allow objective statistical treatment of the data. Principal Component Analysis and Discriminant Analysis of LIBS data resulted in 100\% correct classification of the shooting distance ranges, while color tests resulted in $78.6 \%$ correct classification, $3.6 \%$ misclassification, and $17.8 \%$ inconclusive results. The LIBS method offered improvements over conventional tests such as simplicity, versatility, and reliability. First, the technique is virtually reagent-free, does not require sample preparation and the micro-beam can be scanned across a target in a matter of minutes. Second, the ablation platform can accommodate diverse substrates, and the laser settings can be controlled to offer different solutions, from ultra-fast screening for the identification of IGSR on bullet entrance holes to detailed chemical mappings for shooting distance determinations. Finally, LIBS affords a more reliable and objective presentation of the scientific results in the courtroom. The incorporation of LIBS in forensic laboratories will help to modernize current methodologies and increase the scientific validity of the detection of gunshot residues in crime scene reconstructions.
\end{abstract}

\section{Introduction}

When a firearm is discharged, a mixture of compounds, known as firearm discharge residues (FDR), escape through the openings of the weapon in the form of a vaporous cloud containing particles [1]. The residues can be deposited on the immediate surroundings of the fired gun, such as a shooter's hands or face, or reach target surfaces, including a victim's skin or clothing.

The FDR are composed of inorganic gunshot residues (IGSR) originating from the primer cap, and organic gunshot residues (OGSR) originating from the propellant. A standard-ammunition primer is composed of the initiator lead styphnate $\left(\mathrm{C}_{6} \mathrm{HN}_{3} \mathrm{O}_{8} \mathrm{~Pb}\right)$, the oxidizer barium nitrate $\left(\mathrm{Ba}\left(\mathrm{NO}_{3}\right)_{2}\right)$, and the fuel antimony trisulfide $\left(\mathrm{Sb}_{2} \mathrm{~S}_{3}\right)$. Primers used in non-toxic ammunitions contain other materials including, but not limited to: diazodinitrophenol $\left(\mathrm{C}_{6} \mathrm{H}_{2} \mathrm{~N}_{4} \mathrm{O}_{5}\right)$, tetrazene $\left(\mathrm{H}_{4} \mathrm{~N}_{4}\right)$, potassium nitrate $\left(\mathrm{KNO}_{3}\right)$, aluminum silicate $\left(\mathrm{Al}_{2} \mathrm{SiO}_{5}\right)$, and pentaerythritol tetranitrate $\left(\mathrm{C}_{5} \mathrm{H}_{8} \mathrm{~N}_{4} \mathrm{O}_{12}\right)$ [2]. When the inorganic materials cool down, they condense as spherical particles $(0.5-10 \mathrm{um})$ containing oxides or sulfides of these metals. Other elements originating from the bullet or casing can also contribute to the inorganic profiles. Additionally, the burnt and unburnt propellant (OGSR) contain larger condensates with mixtures of explosives such as nitrocellulose, nitroglycerine, and nitroguanadine [3], stabilizers such as diphenylamine, ethyl centralite, methyl centralite, and sensitizers such as

\footnotetext{
* Corresponding author.

E-mail addresses: chv0002@mix.wvu.edu (C.V.Pyl), oco0001@mix.wvu.edu (O. Ovide), maho@mix.wvu.edu (M. Ho), bayram.yuksel@mail.wvu.edu (B. Yuksel), Tatiana.trejos@mail.wvu.edu (T. Trejos).
} 
nitrotoluenes and dinitrotoluenes [4,5]. Completely burnt OGSR will appear as powder, also known as soot, on a target while unburnt and partially burnt OGSR will be visible propellant particles left on a target.

Consequently, the detection of these residues can provide essential evidence in a firearm related investigation. For instance, the identification of characteristic IGSR particles on an individual's hands can help to identify if the person has fired a gun, or has been in the vicinity of a shooting. The presence of FDR around an orifice can determine if it was produced by a bullet, or help in reconstruction of shooting trajectories. The concentration and pattern of FDR around a bullet hole can help elucidate between a homicide, a suicide, or an accidental shooting. In general, as the muzzle-to-target distance increases, the concentration of IGSR surrounding the entrance orifice decreases. Additionally, the density of particles spreads out, and then decays [6-8].

Currently, the preferred method for bullet hole detection and shooting distance determination uses chemical colorimetric tests to enhance the observation of FDR patterns surrounding a suspected bullet hole. The Modified Griess test reacts into an orange color in the presence of nitrite groups from OGSR, The Sodium Rhodizonate test changes to a pink and then purple color in the presence of barium and lead, respectively. Whereas the chemical colorimetric tests are simple, quick, and sensitive to IGSR components, the major downfall is their lack of specificity. Several studies have shown that an encounter with everyday materials, such as dirt or makeup, could elicit a color change for either one of these tests [9-14]. The colorimetric reaction fades rapidly, making the documentation of observed features challenging. The tests heavily rely on light colored backgrounds and pristine samples in order to accurately visualize the development of the color patterns. Since dirty and dark colored items are prevalent in casework, the tests often require additional transfer of the residues onto photographic or filter paper, which may decrease the efficacy of the presumptive assay. Additionally, the measurements and distance determinations are subjective to the analyst performing the color tests, and therefore, interpretation of results can differ between examiners. The limitations of these color tests decreases the reliability of results when presented in a court of law. Moreover, with the evolving market of heavy metal free ammunitions, there is a demand for a technique with an expanded detection capability.

These challenges have led to evaluate more selective and sensitive instrumental methods to estimate shooting distance. However, to date none of these methods have transitioned from the research stage to the implementation in forensic laboratories. There have been studies that proposed the use of Fourier Transform Infrared Spectroscopy (FTIR) [15], Atomic Force Microscopy (AFM) [16], Atomic Absorption Spectrometry (AA) [17], mili-X-ray Fluorescence Spectrometry (m-XRF) [18-20], and Inductively Couple Plasma-Mass Spectrometry (ICP-MS) [21]. However, these instrumental techniques also possess limitations, including bulk analysis with no spatial information, destruction of sample, low sensitivity for certain elements, high cost and complexity, or long analysis time.

Laser induced breakdown spectroscopy (LIBS) has recently shown promise as a rapid, simple, and versatile detection method for gunshot residues [22-30]. A LIBS instrument operates with a pulsed laser that interacts with the surface of the material and creates a temporal microplasma. The species in the material then vaporize, and atomic and ionic forms are excited. As the plasma begins to cool, the excited species return to their ground states, causing emissions of light at specific wavelengths. This emission is then captured by a spectrograph. [31].

The use of LIBS as an exploratory method in visualizing gunshot residues patterns on white clothing, by chemical imaging, was recently investigated by Lopez et al. and serves as a proof of concept [32]. However, the scanning method selected by the authors was time consuming $(\sim 3 \mathrm{~h} /$ sample), required indirect analysis on an adhesive support, and did not attempt to address the issues of realistic clothing substrates submitted as evidence. In this study, we propose an improved LIBS method that allows direct sampling from the substrate by using a custom-made sampling stage, and validation of the method using a variety of firearms, ammunition and patterned and colored substrates. Statistical methods, including principal component analysis (PCA) and multivariate discriminant analysis were used as tools in the classification of shooting distances of unknown samples. The performance of the proposed method is compared to standard color assays currently used at crime laboratories. The aim of the following study was to develop a more objective, reliable and modern approach to identify bullet holes and estimate shooting distance using LIBS.

\section{Materials and methods}

\subsection{Firearms and ammunitions}

A $9 \mathrm{~mm}$ Springfield XD9 firearm (manufactured in Croatia) and Rossi Revolver 0.357 Magnum firearm (manufactured in Brazil) were used for shootings and sample collections. The ammunition for the Springfield XD9 consisted of Starline brass $9 \mathrm{~mm}$ luger cartridge cases reloaded with Remington $1 \frac{1}{2}$ small pistol primers, Winchester 231 grain powder, and Speer $9 \mathrm{~mm}$ total metal jacket (TMJ) bullets. The ammunition used for the Rossi Revolver was 38 Special 158 grain with a lead round nose bullet.

\subsection{Sample preparation and collection}

Fabric in varying colors (patterned and plain grey, orange, maroon, navy, black, all $100 \%$ cotton) was obtained from a retail store. The fabric was cut into $20 \mathrm{~cm}$ by $27 \mathrm{~cm}$ rectangles. All samples were placed in manila folders, with a white piece of copy paper on either side, and then folded in pre-labeled clean paper. The prepared samples were stored in a sealed plastic container at the laboratory until shooting and collection.

All shootings were performed at the West Virginia University Forensic and Investigative Sciences ballistics laboratory under controlled environmental conditions. Each fabric sample was hung on a self-healing shooting block using push pins and a clean manila folder support to prevent cross contamination. The distance from the muzzle of the firearm to the sample was set using a measuring tape and floor markers before each shooting. It was ensured that the individual handling and storing the samples was not the same individual performing the shootings.

A total of 45 samples (15 grey, 30 patterned) were shot at known distances and used as calibration sets. The distances used for calibration purposes were contact, 6 in., 12 in., 24 in., and 36 in.; each distance was repeated three times for both grey and patterned fabrics. The grey calibration set was shot with pistol, while the pattern sets were shot with a pistol and a revolver. A total of 28 unknown samples were collected; 8 grey, 8 patterned, 3 orange, 3 maroon, 3 navy, and 3 black. The analyst was blind to the true distance of the unknown samples while performing testing.

Nitrile gloves (Fischer Scientific; NH) and lab coats (Fischer Scientific; NH) were worn during sample handling, preparation, collection, and analysis. Gloves were changed between handling each new sample. Blank controls were analyzed in triplicate for each fabric type to evaluate any potential interferences.

\subsection{Experimental setup}

The experimental setup was designed to allow for subsequent analysis on the same fabric sample, first by LIBS, then by traditional physical examination, and Modified Griess and Sodium Rhodizonate chemical color tests. In development of the sequence of experiments, practicality of analysis and concern for sample destruction were also considered. The ablation stage was moved fast while the laser was fired across the fabric's surface. The stage speed allowed the laser-GSR interaction while minimizing textile's damage and disturbance of 


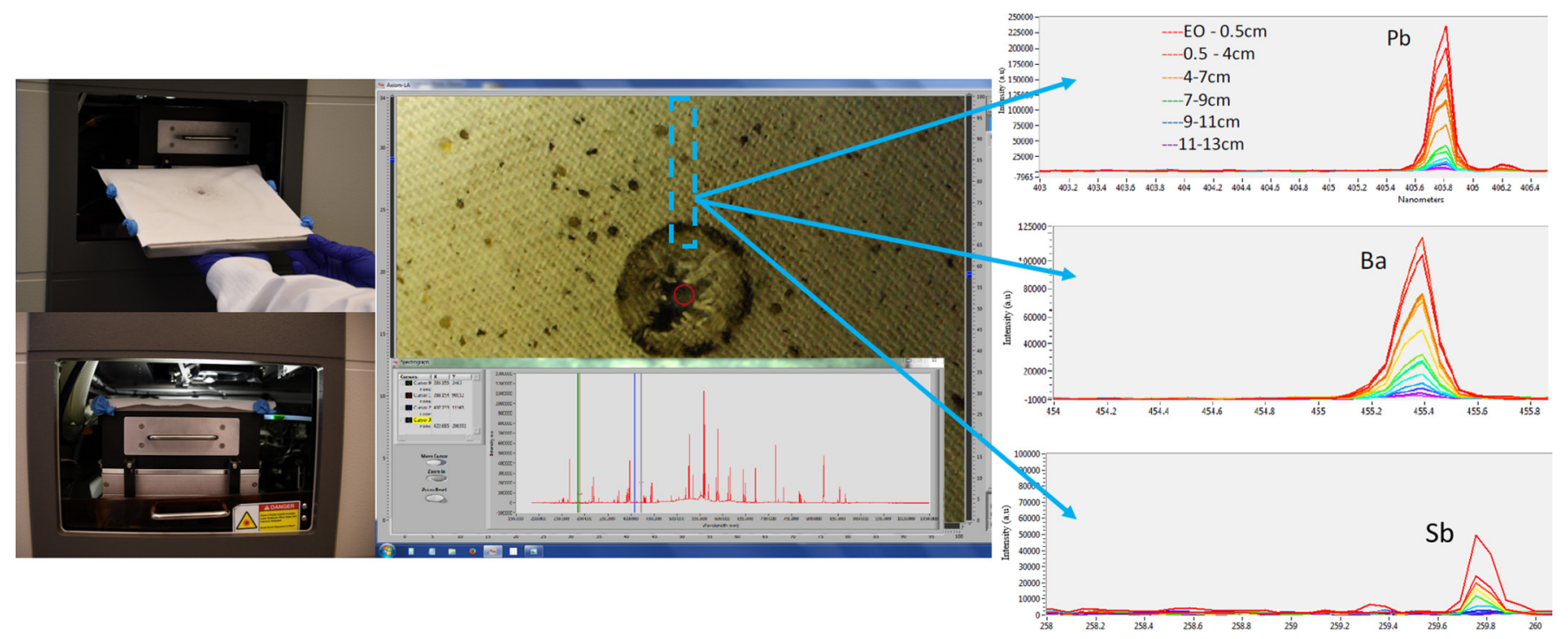

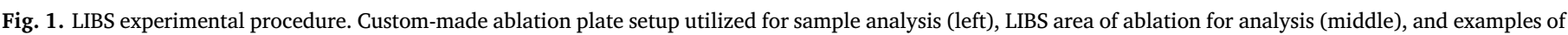
spectra obtained for elements of interest (right, from top to bottom: Pb (405.8 nm, I), Ba (493.4 nm, II), Sb (259.8 nm, I)).

surrounding GSR patterns. The removal of soot and GSR were negligible in comparison to the overall macro-pattern distribution of GSR around the entrance hole. Indeed, the damage left on the substrate was almost invisible to the naked eye.

\subsubsection{LIBS instrumentation, parameters, and method}

A J200 Tandem Model (Applied Spectra; Freemont, CA) LIBS operating with a $266 \mathrm{~nm} 10 \mathrm{~ns}$ Neodymium doped Yttrium Aluminum Garnet laser and 6 channel Czerny-Turner spectrometer was used for analysis. The clothing samples were secured to a custom-made ablation stage that sits on top of the cell in the ablation chamber, with a noninterfering double sided adhesive tape (Fig. 1). The extra length of the fabric was secured to the metal ablation stage using magnets. The stage was covered with benchkote paper (Fisher Scientific, $\mathrm{NH}$ ) to prevent contamination from the metal surface. With the camera focused on the area closest to the bullet hole, the laser was scanned across the fabric using a continuous straight ablation line of $100 \mu \mathrm{m}$ by $5 \mathrm{~mm}$, and the signal was accumulated for 83 shots. The laser was fired with an irradiance of $9.4 \mathrm{E}^{9} \mathrm{~W} / \mathrm{cm}^{2}$, with a frequency of $5 \mathrm{~Hz}$, and a velocity of $0.3 \mathrm{~mm} / \mathrm{s}$. This ablation pattern was repeated 26 consecutive times resulting in a total ablation line of $130 \mathrm{~mm}$ away from the center of the bullet hole.

A Box-Behnken response surface experimental design was used to find ablation parameters that maximize SNR while minimize samples to sample variance (precision). The optimal parameters and ablation pattern were tested on standards prepared by spiking Whatman paper \#42 (Fischer Scientific, $\mathrm{NH}$ ) and white cotton fabrics with $50 \mu \mathrm{g}$ to $50 \mathrm{ng}$ of $\mathrm{Pb}, \mathrm{Ba}$, and $\mathrm{Sb}$, and dried before analysis. These known concentration solutions were used to determine the mass deposited on the substrate and limits of detection for elements of interest. The limits of detection were calculated using three times the standard deviation of the background and dividing it by the slope of the calibration curve, as recommended in literature [31,33], then confirming by measuring standard samples at concentrations near the expected LOD. A preliminary validation was then conducted on a set of fifteen white cotton fabric samples, shot at known distances.

\subsubsection{LIBS data analysis}

Aurora software (Applied Spectra; Freemont, CA) was used for data pre-processing, including background subtraction, peak identification, and peak area integration. The selected elemental peaks of interest were $\mathrm{Sb}(259.8 \mathrm{~nm}, \mathrm{I}), \mathrm{Pb}(405.8 \mathrm{~nm}, \mathrm{I})$, and $\mathrm{Ba}(493.4 \mathrm{~nm}, \mathrm{II})$. Additional emission lines were used for confirmation $(\mathrm{Pb}(368.3 \mathrm{~nm}, \mathrm{I})$, Ba (455.4 nm, II), Ba (553.5 nm, I), Ba (614.1 nm, II), Ba (705.9 nm, I), and $\mathrm{Sb}(252.8 \mathrm{~nm}, \mathrm{I})$ ). Other emission lines, including $\mathrm{Al}$ (396.1 nm, I), Al $(309.3 \mathrm{~nm}, \mathrm{I}), \mathrm{Cu}(327.4 \mathrm{~nm}, \mathrm{I})$ and $\mathrm{Cu}(324.7 \mathrm{~nm}, \mathrm{I})$, were also monitored. The integrated spectral data was initially analyzed using Excel 2016 (version 15.24, Microsoft Corporation). Chemical maps and multivariate statistical methods, including principal component analysis and discriminant analysis, were performed on JMP Pro (version 14.0.0; SAS Institute Inc., NC).

\subsubsection{Color tests and physical examination}

The procedures for the Modified Griess test and Sodium Rhodizonate test, described by Dillon, were followed to perform chemical color tests on all clothing samples [34,35]. The distances determined by the color tests were used to cross validate the results determined by LIBS analysis. Limits of detection of color tests were conducted by depositing $100 \mu \mathrm{L}$ of solutions of known concentrations ( 1000 to $200 \mu \mathrm{g} / \mathrm{mL}$ ) on Whatman paper and let it dry. The deposited dried amounts ranged therefore from $100 \mu \mathrm{g}$ to $2 \mu \mathrm{g}$. The limit of detection was estimated as the lowest deposited amount that produced a color reaction detectable to the naked eye. The experiment was conducted in four replicates and repeated in three different days by three different analysts.

2.3.3.1. Color test reagents. The Modified Griess test was performed using sulfanilic acid (Lot \# BCBQ1007V; Sigma Aldrich, MO), alphanaphthol (Lot \# 10190898; Alfa Aesar, MA), sodium nitrite (Lot \# A0267857, Acros Organics-Thermo Fisher Scientific, MA), acetic acid (Lot \# 171289; Fischer Scientific, NH), methanol (Lot \# 170983; Fisher Chemical, PA), and desensitized HP Everyday Photographic Paper (Model \#Q8723A, Palo Alto, CA). The Sodium Rhodizonate test was performed using sodium rhodizonate (Lot \# BCBR0492V, Sigma Aldrich, MO), sodium bitartrate (Lot \# BCBR3492V, Sigma Aldrich, MO), tartaric acid (Lot\# Y04A021, Alfa Aesar, MA), hydrochloric acid (Lot \# 167045, Fisher Scientific, NH), Whatman paper \#42 (Fisher Scientific, NH), and Search Power Spray Units (Sirchie Acquisition Company LLC, NC). All experiments were conducted in a Safeaire ${ }^{\circledR}$ ventilated fume hood (Fisher Hamilton, Manitowoc, WI).

2.3.3.2. Physical measurements. Photographic documentation and physical measurements [36] were taken on the clothing samples before and after color testing. The length and width of the bullet hole 
and soot pattern were measured. The number of visible gun powder particles on the sample were counted as well as the distance of the particles from the bullet hole. After the Modified Griess test, the area of the orange color present on the photographic paper and the area of the color deposited by the gunpowder particles were measured using ImageJ (version 1.8.0.112, National Institutes of Health, MD). After application of the Sodium Rhodizonate test, the area of the purple color was also measured using the same ImageJ program.

\section{Results and discussion}

One of the main objectives of this study was to evaluate if LIBS can be used as an analytical approach to modernize current presumptive testing used for bullet hole identifications and shooting distance estimations. Consequently, all samples measured by LIBS were also analyzed by the conventional analytical protocol used at crime laboratories, including physical examination and color tests.

\subsection{Firing distance prediction using LIBS analysis and multivariate methods}

\subsubsection{LIBS Method optimization and assessment of selectivity and limits of} detection

The Box Behnken response surface experimental design was used to determine the optimal ablation parameters. Four levels were assessed, each with three different ranks (low, medium, high); pulse frequency $(3 \mathrm{~Hz}, 5 \mathrm{~Hz}, 10 \mathrm{~Hz})$, laser energy $(40 \%, 60 \%, 80 \%)$, gate delay $(0.2 \mu \mathrm{s}$, $0.5 \mu \mathrm{s}, 1.0 \mu \mathrm{s})$, and stage velocity $(0.2 \mathrm{~mm} / \mathrm{s}, 0.3 \mathrm{~mm} / \mathrm{s}, 0.4 \mathrm{~mm} / \mathrm{s})$. The best signal to noise, and least destruction of sample, was accomplished by using a gate delay of $0.5 \mu \mathrm{s}$, firing the laser at $40 \%$ energy $(6 \mathrm{~mJ})$ with a repetition rate of $5 \mathrm{~Hz}$, a spot size of $100 \mu \mathrm{m}$, and accumulating the signal while scanning the laser $5 \mathrm{~mm}$ across the sample at a speed of $0.3 \mathrm{~mm} / \mathrm{s}$. The same pattern was repeated 26 times, moving straight from the bullet hole to ensure the entire GSR distribution pattern was captured during analysis. Using this method, time of analysis for one sample was, on average, $30 \mathrm{~min}$.

Background spectra were taken in triplicate for each fabric type to assess any interfering elemental emission lines at wavelengths of interest. Except for a Silicon $(252.8 \mathrm{~nm}$, I) interference with $\mathrm{Sb}$ $(252.8 \mathrm{~nm})$, no interferences were observed. Antimony's second emission line at $259.8 \mathrm{~nm}$ did not display selectivity issues (Fig. 2).

Limits of detection (LOD) of LIBS and color tests were compared by depositing known amounts of elements of interest on Whatman paper and letting it dry prior ablation. Fig. 3 illustrates the superior sensitivity of LIBS over color tests. LIBS absolute limits of detection were 2 to 4 orders of magnitude better than color tests, for lead and barium respectively. Its recognized that color test's limits of detection could be improved by instrumental detection of the color rather than relying on visual detection. Nonetheless, the human eye detection was preferred as more representative of the method used by forensic examiners. In order to account for subjectivity in the color detection, the experiment was repeated by three different analysis and conducted in replicates. The reported values represent the average results and respective standard deviations. Moreover, LIBS can detect more elements associated with FDR, such as antimony, expanding current capabilities of chemical color tests in the field. (Table 1).

\subsubsection{Chemical maps for shooting distance determinations}

Shooting distances for the calibration curves were chosen to represent gunshot residue patterns that are commonly associated with contact, close range (6 in. \& 12 in.), and long range ( 24 in. \& 36 in.) shootings. The integrated spectral data collected from shot fabrics was pre-processed to visualize the spatial changes on elemental composition relative to the firing distance. A bar graph of intensity versus ablation distance from bullet hole ( $\mathrm{mm}$ ) was created for each element of interest $(\mathrm{Pb}, \mathrm{Ba}, \mathrm{Sb})$, representing the accumulated area intensity of each $5 \mathrm{~mm}$ increment as the laser moved away from the orifice. For each of the calibration sets, all distances (contact, 6 in., 12 in., 24 in., and 36 in.) were displayed on the same graph to illustrate the difference in chemical intensity patterns. Fig. 4 shows a representation of $\mathrm{Pb}(405.8 \mathrm{~nm})$ for one set of grey calibration samples. Similar graphs can be seen for $\mathrm{Ba}(493.4 \mathrm{~nm})$ and $\mathrm{Sb}(259.8 \mathrm{~nm})$ in Figs. S1 and S2 in the supplemental section. The contact sample shows the highest initial intensity closest to the bullet hole, with a sharp decline in intensity that levels out around $4 \mathrm{~mm}$. The short range distance samples (6 in. \& 12 in.) show lower initial intensities but have a more gradually decline before leveling out at $8.5 \mathrm{~mm}$. Long range distances ( $24 \mathrm{in}$. \& $36 \mathrm{in}$.) have consistent low intensity levels from the entrance hole to the end of the fabric, with slightly higher levels for 24 in. It should be noted that some contact samples had lower initial intensities due to the large star shaped patterned surrounding the bullet hole (Fig. S5a top left image). It is suspected that the large opening allowed for GSR to travel through the fabric, instead of being imbedded at the entry hole. To account for this issue when performing multivariate statistical methods, bullet hole dimensions were added to each sample as an additional qualifier.

As a supplementary and more advanced visualization of the data, chemical heat maps were made, using JMP Pro 14, for each element of interest. For instance, Fig. 5 shows heat maps for the distribution of $\mathrm{Ba}$ (493.4 nm, II) at 4 different distances. The intensity data obtained for each sample was expanded $360^{\circ}$ to create concentric, uniform heat maps representative of the GSR pattern distributed around the bullet hole. Similar heat maps can be seen for $\mathrm{Ba}(493.4 \mathrm{~nm})$ and $\mathrm{Sb}$ $(259.8 \mathrm{~nm})$ in Figs. S3 and S4, respectively. The heat maps allow for a permanent visualization of IGSR distribution patterns. Similar to color tests, the darker colors represent a higher concentration of the analyte and the lighter colors represent lower concentrations of the analyte. Unlike color tests, the chemical maps contain spectral and numerical data, resulting in a more objective representation of the GSR pattern. Additionally, LIBS allows for the creation of heats maps for all elements of interest (lead, barium and antimony), which is unable to be achieved using color tests that are limited to nitrites and lead detection. These chemical maps are expected to facilitate explanation of the results to the trier of fact.

\subsubsection{Multivariate methods}

Statistical approaches to classification of unknown distances included principal component analysis and multivariate discriminant analysis. As a first step, PCA plots were constructed using the spectral intensities found for the entirety of the $5 \mathrm{~mm}$ ablation increments; $130 \mathrm{~mm}$ total. Each increment had numeric outputs from the accumulated integrated areas of the multiple elements and emission lines. All three replicates of calibration curve samples were used in the construction of the PCA plots. Grey calibration curve sets were assessed with 3 grey, 3 orange, 3 maroon, 3 navy, and 3 black unknown samples. Patterned calibration curve samples were assessed with 5 patterned and 5 grey unknown samples. When using the full $130 \mathrm{~mm}, 71.4 \%, 76.5 \%$, and $83.3 \%$ of the variation of the grey and patterned (pistol and revolver) data sets were captured, respectively. In an attempt to increase the amount of variability captured, the data was reassessed and it was determined that any data after $100 \mathrm{~mm}$ of ablation provided little information. The elemental intensities, for all distances, were too similar past this ablation distance, and only added unnecessary noise to the data set. When the PCA plots were reconstructed with the reduced data set and included the bullet hole qualifier, the variability captured increased to $79.1 \%, 83.8 \%$, and $90 \%$ for the grey and patterned (pistol and revolver) calibration sets, respectively.

The first two principal components, along with the bullet hole qualifier, were used as the variables to perform discriminant analysis and construct a canonical plot (Fig. 6). Discriminant analysis is a supervised statistical procedure that classifies unknown samples into a certain group. Discriminant analysis uses a training data set to estimate a discriminant function, or canonical, that maximizes the distance between each individual data point and the central data point while 

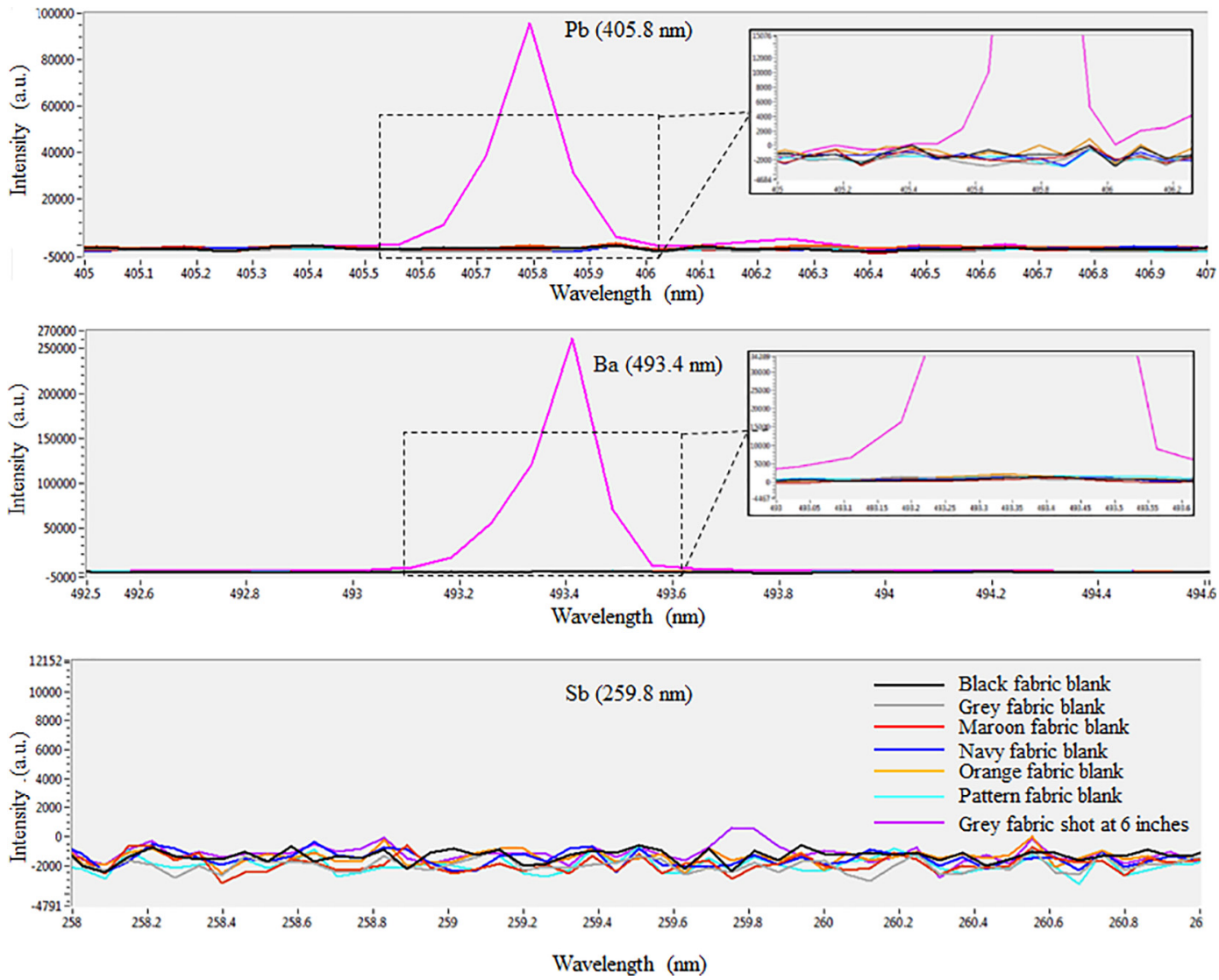

Fig. 2. LIBS spectra of a clothing shot at 6 in. away and background spectra of the textiles prior shooting illustrating no interferences for elements of interest from negative control fabric samples.

minimizing the scatter within each category. A probability is then calculated to predict a category for each case. The probability that an unknown case belongs to a certain group is based on the calculation of relative Mahalanobis' distances, measuring the distance to the centroid of each group. The canonical plot in Fig. 6 shows the points and multivariate least-squares means on the first two canonical variables that best separate the groups. A 95\% confidence level ellipse is plotted for each mean. If two groups differ significantly, the confidence ellipses tend not to intersect. Another ellipse denoting a $50 \%$ contour is plotted for each group. This depicts a region of the plot that contains
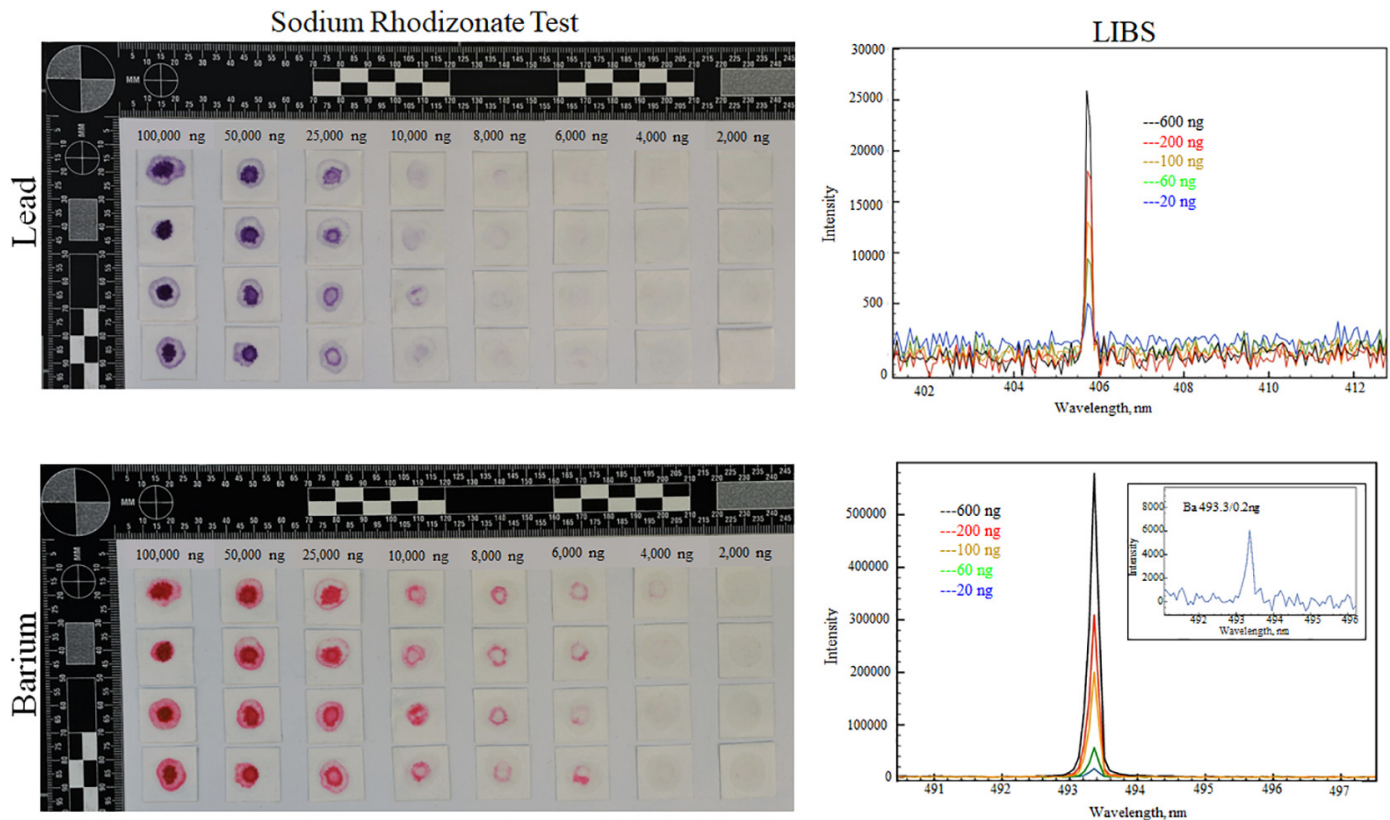

Fig. 3. Results of limits of detection tests for the sodium rhodizonate assay (left), and LIBS (right), for lead (top) and barium (bottom), respectively. The inset shows a zoomed signal for $\mathrm{Ba}(493 \mathrm{~nm})$ at the limit of detection of $0.2 \mathrm{ng}$. 
Table 1

Comparison of limits of detection for Sodium Rhodizonate test and LIBS method.

\begin{tabular}{|c|c|c|c|}
\hline & LIBS & & \\
\hline Species & Wavelength (nm) & LOD (ng) & Sodium Rhodizonate (ng) \\
\hline \multirow[t]{2}{*}{ Lead $(\mathrm{Pb})$} & $368.3(\mathrm{I})$ & $70 \pm 3$ & \multirow[t]{2}{*}{$7000 \pm 1000$} \\
\hline & 405.7 (I) & $30 \pm 4$ & \\
\hline \multirow[t]{5}{*}{ Barium (Ba) } & 455.4. (II) & $0.70 \pm 0.08$ & \multirow[t]{5}{*}{$5000 \pm 1000$} \\
\hline & 493.4 (II) & $0.20 \pm 0.02$ & \\
\hline & 553.5 (I) & $1.1 \pm 0.04$ & \\
\hline & 614.1 (II) & $1.4 \pm 0.04$ & \\
\hline & 705.9 (I) & $2.1 \pm 0.1$ & \\
\hline \multirow[t]{2}{*}{ Antimony (Sb) } & $252.8(\mathrm{I})$ & $440 \pm 80$ & \multirow[t]{2}{*}{ N/A } \\
\hline & 259.8 (I) & $220 \pm 13$ & \\
\hline \multirow[t]{2}{*}{ Copper $(\mathrm{Cu})$} & 324.7 (I) & $40 \pm 6$ & \multirow[t]{2}{*}{ N/A } \\
\hline & 327.4 (I) & $20 \pm 4$ & \\
\hline \multirow[t]{2}{*}{ Aluminum (Al) } & 309.3 (I) & $50 \pm 6$ & \multirow[t]{2}{*}{ N/A } \\
\hline & 396.2 (I) & $30 \pm 2$ & \\
\hline
\end{tabular}

approximately $50 \%$ of the observations. Fig. 6 shows a good separation between contact, 24 and 36 in. groups, while 6 in. and 12 in. tend to be relatively closer.

To test the fitness of the model, the misclassification rates of a training and a validation should be low and comparable. The training set is used to predict the responses for the observations, while the validation set is composed of samples shot at unknown distances to test the group prediction. In our study, all unknown samples in this set were shot at $10 \mathrm{in}$. away from the target and therefore they should be correctly classified by the model in the 6-12 in. range. A misclassification occurs when the determined distance range does not include the true distance or the determined distance range was too large. A distance range was not needed to classify an unknown sample that was a contact shot due to the distinctive characteristic of the star-shaped pattern.

To compensate for the small number of samples in this study, regularized discriminant analysis was applied. In both cases, the calibration curve samples were used as the training set and the unknown samples were used as the validation set. Classifications of unknowns were determined by the first and second predictions made by the discriminant analysis model. Sixteen unknown samples (grey, orange, maroon, navy, black, pattern) had a first prediction of $12 \mathrm{in}$. and a second prediction of $6 \mathrm{in}$. Therefore, the determined distance range for these 16 samples was 6 in.-12 in. (close range shot) with an emphasis closer to $12 \mathrm{in}$. The model predicted 7 unknown samples (patterned) to be $24 \mathrm{in}$. with a second prediction of $36 \mathrm{in}$. and 5 samples (grey and patterned) to be $36 \mathrm{in}$. with a second prediction of $24 \mathrm{in}$. No misclassifications of LIBS spectral patterns were found in the validation sets.

\subsection{Firing distance predictions using color tests}

\subsubsection{Interpretation of physical and visual examination and chemical color} tests

A typical physical examination of a fired clothing includes the comparison of bullet hole dimensions, soot dimensions, and gun powder distribution and density around the orifice. A calibration set is normally shot with the same suspect firearm and ammunition on similar fabrics as the questioned item. Then, the observations are visually compared, and the examiners decides to what known distance the questioned sample is most similar. The process unfortunately is very subjective and dependent of the examiner experience. Table S1 illustrates the measurements taken for calibration sets and unknown samples (grey, orange, maroon, navy, and black). Darker fabrics including the maroon, navy, black, and patterned, made it difficult to accurately complete physical measurements. The wide variability of between and within physical properties for these samples resulted in a large number of misclassifications and inconclusive results $(28.6 \%$ and $35.7 \%$, respectively).

All 73 samples in this study were subjected to both the Modified Griess test and the Sodium Rhodizonate test (Table S2). Positive controls were completed on the corners of the prepared Griess test sheets prior to analysis on a sample. It should be noted that LIBS ablations did not interfere with Modified Griess distribution patterns. The laser moving quickly across the surface of the sample did not disrupt surrounding soot or gunpowder particles. A microscopic ablation line, with

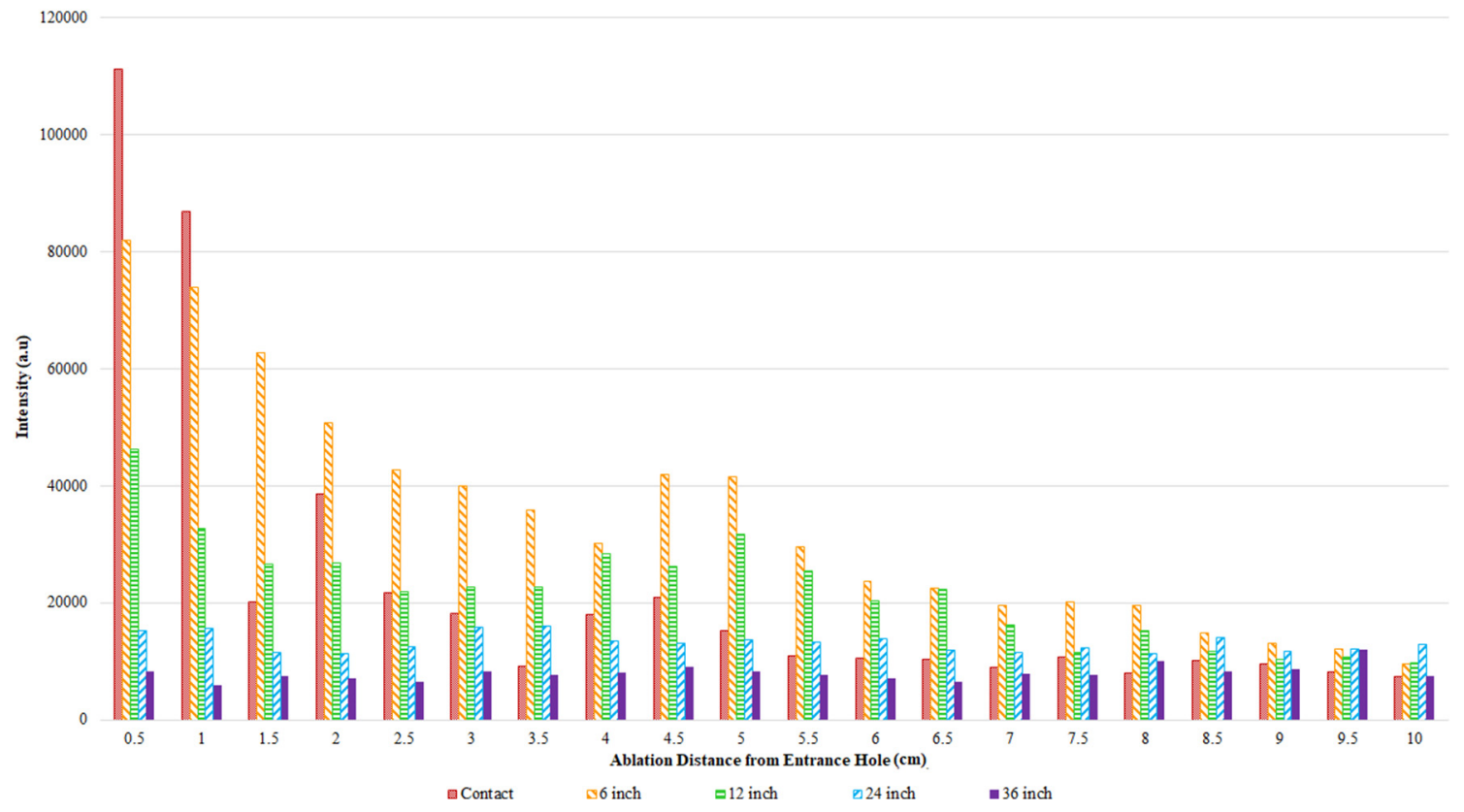

Fig. 4. Bar graph representing intensity patterns for $\mathrm{Pb}(405.8 \mathrm{~nm})$ for a grey fabric calibration set. From left to right, the ablation from the entrance hole begins at $0.5 \mathrm{~cm}$ and increases by $0.5 \mathrm{~cm}$ until ending at $10 \mathrm{~cm}$. The legend represents the different shooting distance used for calibration (checkered red $=$ contact, diagonal yellow $=6 \mathrm{in}$., horizontal green $=12 \mathrm{in}$., diagonal blue $=24 \mathrm{in}$., solid purple $=36 \mathrm{in}$.). (For interpretation of the references to color in this figure legend, the reader is referred to the web version of this article). 

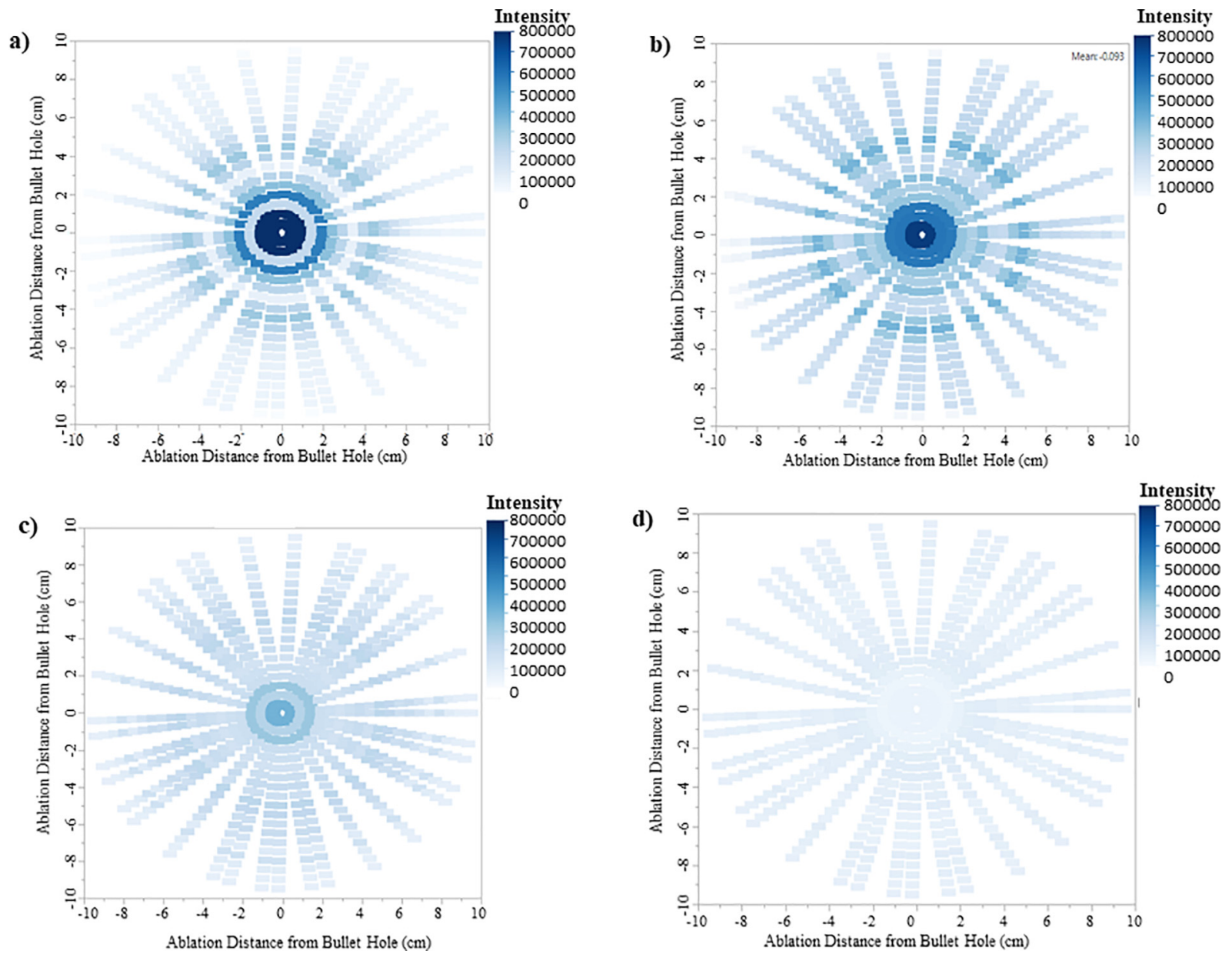

Fig. 5. Heat maps of $\mathrm{Ba}(493.4 \mathrm{~nm})$ obtained from a grey-fabric calibration set (a) contact; (b) 6 in.; (c) 12 in.; (d) 24 in.

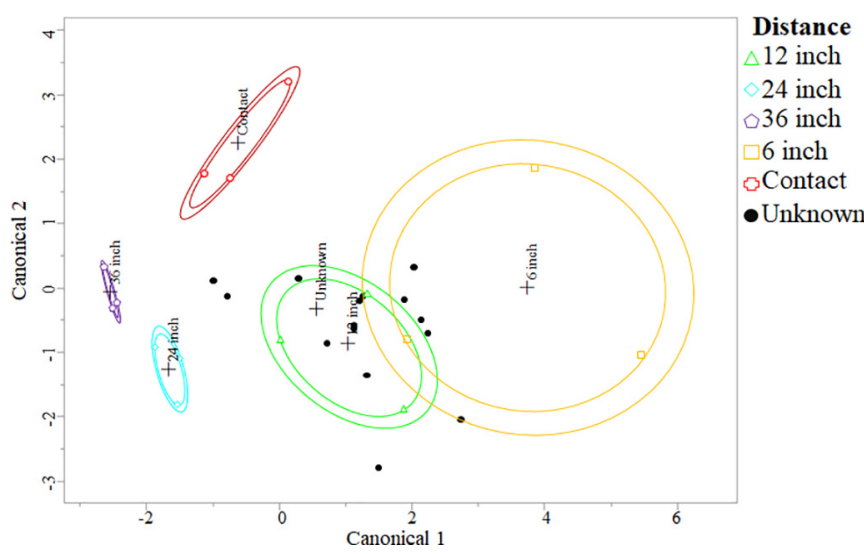

Fig. 6. Canonical plot for grey calibration samples with grey unknowns, orange unknowns, maroon unknowns, navy unknowns, and black unknowns (3 replicates each). All unknowns have a true shooting distance of $10 \mathrm{in}$. (+ represents the multivariate mean for each shooting distance, outer ellipses represent 95\% confidence interval for each shooting distance, and inner ellipses represent a $50 \%$ contour of observations for each shooting distance. (For interpretation of the references to color in this figure legend, the reader is referred to the web version of this article).

a width of $100 \mu \mathrm{m}$, is created only on the fabric's surface but does not penetrate the fabric. Additionally, this is confirmed in Fig. S7a. where no disruption in pattern can be seen in the Modified Griess test results. The calibration curve samples showed consistent Griess test results for all replicate samples. Performing the Griess test on the orange and maroon colored fabrics helped to enhance the gunshot residue patterns on the fabric that were previously masked by the background color of the fabric. However, the navy and black fabrics reacted with the reagents of the Griess test and caused an orange color to appear on the entirety of the photographic paper (Fig. S7b). The interference was confirmed by analyzing blank negative control samples of the black and navy fabrics by the Griess test, where an orange reaction was observed even in the absence of FDR. While the color produced by the navy fabric was fainter than that of the black fabric, both interferences compromised the measurements. The fabric interference and lack of reproducibility prevented a reliable distance estimation for black or navy samples.

To keep the integrity of the sample intact, the Sodium Rhodizonate test was performed using the transfer method with Whatman paper \#42. The Sodium Rhodizonate test was not sensitive enough for the type of ammunition employed in the pistol experiments. This is expected to be a result of the low concentrations of lead in the primer cap which produced a pattern of lead residues below the color test LOD (Fig. 3). Since the ammunition used a Total Metal Jacket (TMJ), the main source of lead is anticipated to originate from the primer. Ongoing primer characterization studies in our group have shown that the Remington primer used in the pistol ammunition had lower lead concentration than other standard leaded ammunitions. In general, color test measurements showed a large variability, and limitations of sensitivity and selectivity that prevented consistent estimation of shooting distances. 
Table 2

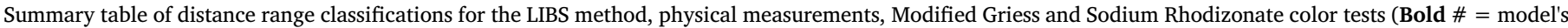
first prediction, Non-bold \# = model's second prediction).

\begin{tabular}{|c|c|c|c|c|c|c|c|}
\hline Unknown sample name & $\begin{array}{l}\text { LIBS/discriminant } \\
\text { analysis distance range } \\
\text { classification }\end{array}$ & $\begin{array}{l}\text { Actual } \\
\text { Distance }\end{array}$ & $\begin{array}{l}\text { Correct distance range } \\
\text { by LIBS/discriminant } \\
\text { analysis? }\end{array}$ & $\begin{array}{l}\text { Visual/physical } \\
\text { distance range } \\
\text { classification }\end{array}$ & $\begin{array}{l}\text { Correct distance } \\
\text { range by visual/ } \\
\text { physical? }\end{array}$ & $\begin{array}{l}\text { Color tests distance } \\
\text { range classification }\end{array}$ & $\begin{array}{l}\text { Correct distance } \\
\text { range by color } \\
\text { tests }\end{array}$ \\
\hline Unknown 1 Grey (p) & 6-12 in. & $10 \mathrm{in.}$ & Yes & $6-24$ in. & Range too large & $6-12$ in. & Yes \\
\hline Unknown 2 Grey (P) & 6-12 in. & 10 in. & Yes & $12-24$ in. & No & $6-36$ in. & Range too large \\
\hline Unknown 3 Grey (P) & 6-12 in. & $10 \mathrm{in.}$ & Yes & $6-24$ in. & Range too large & $6-12$ in. & Yes \\
\hline Unknown 4 Orange $(\mathrm{P})$ & 6-12 in. & $10 \mathrm{in.}$ & Yes & $6-24$ in. & Range too large & 6-12 in. & Yes \\
\hline Unknown 5 Orange (P) & 6-12 in. & $10 \mathrm{in.}$ & Yes & $12-24$ in. & No & $6-36$ in. & Range too large \\
\hline Unknown 6 Orange $(\mathrm{P})$ & 6-12 in. & $10 \mathrm{in.}$ & Yes & $6-24$ in. & Range too large & $6-12$ in. & Yes \\
\hline Unknown 7 Maroon (P) & 6-12 in. & 10 in. & Yes & $6-24$ in. & Range too large & $6-12$ in. & Yes \\
\hline Unknown 8 Maroon $(\mathrm{P})$ & 6-12 in. & $10 \mathrm{in.}$ & Yes & $12-24$ in. & No & $6-36$ in. & Range too large \\
\hline Unknown 9 Maroon (P) & 6-12 in. & $10 \mathrm{in.}$ & Yes & $6-24$ in. & Range too large & $6-12$ in. & Yes \\
\hline Unknown 10 Navy (P) & 6-12 in. & $10 \mathrm{in.}$ & Yes & $6-24$ in. & Range too large & $6-12$ in. & Yes \\
\hline Unknown 11 Navy (P) & 6-12 in. & 10 in. & Yes & $12-24$ in. & No & $6-36$ in. & Range too large \\
\hline Unknown 12 Navy (P) & 6-12 in. & $10 \mathrm{in.}$ & Yes & $6-24$ in. & Range too large & $6-12$ in. & Yes \\
\hline Unknown 13 Black (P) & 6-12 in. & $10 \mathrm{in.}$ & Yes & $6-24$ in. & Range too large & $6-12$ in. & Yes \\
\hline Unknown 14 Black (P) & 6-12 in. & $10 \mathrm{in.}$ & Yes & $12-24$ in. & No & $6-36$ in. & Range too large \\
\hline Unknown 15 Black (P) & 6-12 in. & 10 in. & Yes & $6-24$ in. & Range too large & $6-12$ in. & Yes \\
\hline Unknown 16 Grey (P) & $24-36$ in. & 24 in. & Yes & $24-36$ in. & Yes & $24-36$ in. & Yes \\
\hline Unknown 17 Grey (P) & 24-36 in. & $24 \mathrm{in.}$ & Yes & $24-36$ in. & Yes & $24-36$ in. & Yes \\
\hline Unknown 18 Grey (P) & 24-36 in. & $24 \mathrm{in.}$ & Yes & $24-36$ in. & Yes & $24-36$ in. & Yes \\
\hline Unknown 19 Grey (P) & 24-36 in. & $24 \mathrm{in.}$ & Yes & $24-36$ in. & Yes & $24-36$ in. & Yes \\
\hline Unknown 20 Grey (P) & $24-36$ in. & $24 \mathrm{in.}$ & Yes & $24-36$ in. & Yes & $24-36$ in. & Yes \\
\hline $\begin{array}{l}\text { Unknown } 21 \text { Patterned } \\
\text { (P) }\end{array}$ & 6-12 in. & $12 \mathrm{in.}$ & Yes & $24-36$ in. & No & $24-36$ in. & No \\
\hline $\begin{array}{l}\text { Unknown } 22 \text { Patterned } \\
\text { (P) }\end{array}$ & 24-36 in. & $24 \mathrm{in.}$ & Yes & 24-36 in. & Yes & 24-36 in. & Yes \\
\hline $\begin{array}{l}\text { Unknown } 23 \text { Patterned } \\
\text { (P) }\end{array}$ & 24-36 in. & $24 \mathrm{in.}$ & Yes & $24-36$ in. & Yes & $24-36$ in. & Yes \\
\hline $\begin{array}{l}\text { Unknown } 24 \text { Patterned } \\
\text { (P) }\end{array}$ & 24-36 in. & $24 \mathrm{in.}$ & Yes & $24-36$ in. & Yes & 24-36 in. & Yes \\
\hline $\begin{array}{l}\text { Unknown } 25 \text { Patterned } \\
\text { (P) }\end{array}$ & 24-36 in. & 24 in. & Yes & $24-36$ in. & Yes & $24-36$ in. & Yes \\
\hline $\begin{array}{l}\text { Unknown } 26 \text { Patterned } \\
\text { (R) }\end{array}$ & 24-36 in. & 24 in. & Yes & $12-24$ in. & No & $12-24$ in. & Yes \\
\hline $\begin{array}{l}\text { Unknown } 27 \text { Patterned } \\
\text { (R) }\end{array}$ & 24-36 in. & $24 \mathrm{in.}$ & Yes & 24-36 in. & Yes & 24-36 in. & Yes \\
\hline $\begin{array}{l}\text { Unknown } 28 \text { Patterned } \\
\text { (R) }\end{array}$ & 24-36 in. & $24 \mathrm{in.}$ & Yes & $12-24$ in. & No & $12-24$ in. & Yes \\
\hline $\begin{array}{l}\text { Correct Classification } \\
\text { Rate }\end{array}$ & & & $100 \%$ & & $35.7 \%$ & & $78.6 \%$ \\
\hline
\end{tabular}

\subsection{True shooting distances: assessment of accuracy of the methods}

When reconstructing a crime scene involving a firearm it can be crucial to determine whether a shooting was an accident, a suicide, or a homicide. When deciding between these options, shooting distance determination becomes important; was it a contact, close range, or long range shot? Rather than a specific distance, a range of distances is typically reported. Therefore, the previously classified samples were stated in ranges, rather than one specific distance. A proper classification was considered when the true shooting distance falls into a 1 increment distance range; contact, 6 in.-12 in., 12 in.-24 in., 24 in.-36 in. An incorrect classification was documented when the reported range falls outside the true distance, or the range spans more than one increment. Out of the 28 unknown samples tested in this study, $100 \%$ of them were determined to be correctly classified when using the proposed LIBS method and statistical analyses. On the other hand, only $35.7 \%$ of the samples were correctly classified when relying solely on physical measurements and $78.6 \%$ when using traditional color tests. Table 2 shows a breakdown of the results for each sample by the technique used. The LIBS predictions have bolded numbers representing the model's first predictions and non-bolded numbers for the model's second predictions. The results of this study establish the forensic value of implementing the proposed LIBS method as a technique for shooting distance determinations and GSR pattern visualization in crime laboratories.

\section{Conclusions}

The challenges and limitations associated with the current methods for shooting distance determination emphasize the need for a versatile analytical tool that will enhance the scientific reliability of the detection, observation, and interpretation of GSR patterns on substrates of interest commonly encountered at a crime scene. The purpose of this study was to develop a more objective, reliable, sensitive, and superior approach to determining the distance from which a firearm was discharged. The use of laser-induced breakdown spectroscopy in this discipline has shown to have advantages over current chemical methods, including:

Ease of sample preparation and analysis-the LIBS analytical method does not require any additional preparation of the sample. Additionally, unlike color tests, LIBS does not require the use of fume hood, proper disposal of hazardous waste, preparation of fresh reagents due to their instability, and photographic documentation between the steps of color development.

Minimal destruction of substrate-the substrate of interest is negligibly damaged during the experimental process, which allows for reanalysis or subsequent analysis by other methods if necessary.

Selectivity and multi-elemental detection capabilities-LIBS is capable of detecting multiple elemental emission lines associated with firearm discharge residues (several atomic and ionic emission lines of $\mathrm{Pb}, \mathrm{Ba}, \mathrm{Sb}, \mathrm{Al}, \mathrm{Cu}$ ) simultaneously, improving selectivity and confidence in the results. 
Superior sensitivity-LIBS is able to detect elements associated with GSR at low levels $(0.2 \mathrm{ng}$ to $440 \mathrm{ng}$, depending on the element and emission line) This is essential with the advancement of more environmentally friendly primer caps containing reduced concentrations of heavy metals. Additionally, LIBS is capable of detecting multiple atomic and ionic emission lines for each element, increasing the certainty of its presence while decreasing the rate of false positives.

More objective interpretation of GSR patterns-LIBS has the capability of capturing spatial information which allows for the creation of permanent and objective chemical mapping of GSR patterns on clothing samples and other substrates and the use of statistical tools for prediction of distance.

The application of this study is anticipated to aid in crime scene reconstruction when a firearm is involved in a criminal event and offer more reliable investigative information and defensible results in court. Ongoing research in our group includes using additional realistic crimescene clothing substrates (e.g. bloody samples), as well as, using a similar analytical approach for the identification of bullet holes in surfaces frequently encountered at a crime scene and an extended range of ammunition.

\section{Acknowledgments}

The authors would like to thank Dr. Keith Morris and James Hamilton, for their assistance in the collection of samples at the West Virginia University ballistics laboratory. Dr. Bayram Yuksel would like to acknowledge TUBITAK-BIDEB 2219-International Postdoctoral Research Fellowship Program for financial support".

\section{Appendix A. Supplementary data}

Supplementary data to this article can be found online at https:// doi.org/10.1016/j.sab.2018.12.010.

\section{References}

[1] O. Dalby, D. Butler, J.W. Birkett, Analysis of gunshot residue and associated materials-a review, J. Forensic Sci. 55 (2010) 924-943.

[2] C. Galluzzi, (2006) US Patent No.: 7,056,401 B2, Priming Mixture for Cartridge Primers for Small Firearms.

[3] A.J. Schwoeble, D.L. Exline, Current methods in Forensic Gunshot Residue Analysis, CRC Press, Boca Raton, 2000.

[4] W.A. MacCrehan, M. Bedner, Development of a Smokeless Powder Reference for Propellant and Explosive Analysis for. Sci. Int, 163 (2006), pp. 119-124.

[5] C. Perre, I. Corbin, M. Blas, B.R. McCord, Separation and identification of smokeless gunpowder additives by capillary electrohromatography, J. Chromatogr. A. 1267 (2012) 259-265.

[6] I.C. Stone, V.J. DiMaio, C.S. Petty, Gunshot wounds: visual and analytical procedures, J. Forensic Sci. 23 (1978) 361-367.

[7] V.J. DiMaio, C.S. Petty, I.C. Stone, An experimental study of powder tattooing of the skin, J. Forensic Sci. 21 (1976) 367-372.

[8] R.J. Joling, An overview of firearms identification evidence for attorneys. I: salient features of firearms evidence, J. Forensic Sci. 26 (1981) 153-158.

[9] A. Salvatore, Forensic applications of sodium rhodizonate and hydrochloric acid: a new histological technique for detection of gunshot residues, J. Forensic Sci. 56 (2011) 771-774.

[10] K.H. Chang, P.T. Jayaprakash, C.H. Yew, A.F.L. Abdullah, Gunshot residue analysis and its evidential values: a review, Aust. J. Forensic Sci. 45 (2013) 3-23.

[11] G.M. Ingo, M. D'Uffizi, G. Flalso, G. Bultrini, G. Padeletti, Thermal and microchemical investigation of automotive brake pad wear residues, Thermochim. Acta 418 (2004) 61-68.
[12] E. Vermiej, W. Duvalois, R. Webb, M. Koeberg, Morphology and composition of pyrotechnic residues formed at different levels of confinement, Forensic Sci. Int. 186 (2009) 68-74.

[13] K.L. Kosanke, R.C. Dujay, B.J. Kosanke, Pyrotechnic reaction residue particle analysis, J. Forensic Sci. 51 (2006) 296-302.

[14] S.A. Phillips, Pyrotechnic residues analysis - Detection and analysis of characteristic particles by scanning electron microscopy/energy dispersive spectroscopy, Sci. Justice 41 (2001) 73-80.

[15] S.P. Sharma, S.C. Lahiri, A preliminary investigation into the use of FTIR microscopy as a probe for the identification of bullet entrance holes and the distance of firing, Sci. Justice 49 (2009) 197-204.

[16] Y. Mou, J. Lakadwar, J.W. Rabalais, Evaluation of shooting distance by AFM and FTIR/ATR analysis of GSR, J. Forensic Sci. 53 (2008) 1381-1386.

[17] R. Galiano-Candela, A.P. Colucci, S. Napoli, Determination of firing distance. Lead analysis on the target by Atomic Absorption Spectroscopy (AAS), J. Forensic Sci. 53 (2008) 321-324.

[18] S. Latzel, D. Neime, R. Schuamacher, M. Barth, L. Niewöhner, Shooting distance determination by $\mathrm{m}$-XRF-Examples on spectra interpretation and range estimation, Forensic Sci. Int. 223 (2012) 273-278.

[19] R. Schumacher, M. Barth, D. Neimke, L. Niewöhner, Investigation of gunshot residue patterns using milli-XRF-techniques: First experiences in casework, Proc. SPIE 7729, Scanning Microscopy 2010, 3 June 2010, p. 772917, , https://doi.org/10. $1117 / 12.853852$

[20] A. Berende, D. Neimke, R. Schumacher, M. Barth, A versatile technique for the investigation of gunshot residue patterns on fabrics and other surfaces: m-XRF, J. Forensic Sci. 51 (2006) 1085-1090.

[21] A. Santos, T. Magalhães, D.N. Vieira, A.A. Almeida, A.V. Sousa, Firing distance estimation through the analysis of the gunshot residue deposit pattern around the bullet entrance hole by inductively coupled plasma-mass spectrometry, Am J Forensic Med Pathol 28 (2007) 24-30.

[22] M.J. Silva, J. Cortez, C. Pasquini, R.S. Honorato, A.P.S. Paima, M.F. Pimentel, Gunshot residues: screening analysis by laser-induced breakdown spectroscopy, J. Braz. Chem. Soc. 20 (2009).

[23] C.R. Dockery, S.R. Goode, Laser-induced breakdown spectroscopy for the detection of gunshot residues on the hands of a shooter, Appl. Opt. 42 (2003) 6153-6158.

[24] M.B. Rosenberg, C.R. Dockery, Determining the lifetime of detectable amounts of gunshot residue on the hands of a shooter using Laser-induced breakdown spectroscopy, Appl. Spectrosc. 62 (2008) 1238-1241.

[25] S.R. Goode, C.R. Dockery, M.F. Bachmeyer, A.A. Nieuwland, S.L. Morgan, Detecting Gunshot Residue by Laser Induced Breakdown Spectroscopy, (2018), https://doi. org/10.1364/LIBS.2002.FB2.

[26] J.R. Almirall, S. Umpierrez, W. Castro, I. Gornushkin, J. Winefordner, Forensic elemental analysis of material by laser induced breakdown spectroscopy (LIBS), Proc. SPIE 5778, Sensors, and Command, Control, Communications, and Intelligence (C3I) Technologies for Homeland Security and Homeland Defense IV, 20 May 2005, https://doi.org/10.1117/12.605754.

[27] A. Tarifa, J.R. Almirall, Fast detection and characterization of organic and inorganic gunshot residues on the hands of suspects by CMV-GC-MS and LIBS, Sci. Justice 55 (2015) 168-175.

[28] E. Hondrogiannis, D. Anderson, A.W. Miziolek, The evaluation of a new technology for gunshot residue (GSR) analysis in the field, Proc. SPIE 8726, Next-Generation Spectroscopic Technologies VI, 29 May 2013, p. 87260P, , https://doi.org/10. $1117 / 12.2017900$.

[29] T. Trejos, C. Vander Pyl, K. Menking-Hoggatt, A. Alvarado, L.E. Arroyo, Fast identification of inorganic and organic gunshot residues by LIBS and electrochemical methods, Forensic Chem. 8 (2018) 146-156.

[30] D.W. Hahn, N. Omenetto, Laser-induced breakdown spectroscopy (LIBS), part II: review of instrumental and methodological approaches to material analysis and applications to different fields, Appl. Spectrosc. 66 (2012) 347-419.

[31] V. Palleschi, I. Schechter, A.W. Miziolek (Ed.), Laser-induced Breakdown Spectroscopy (LIBS): Fundamentals and Applications, Cambridge University, 2018.

[32] M. López-López, C. Alvarez-Llamas, J. Pisonero, C. García-Ruiz, N. Bordel, An exploratory study of the potential use of LIBS for visualizing gunshot residue patterns, Forensic Sci. Int. 273 (2017) 124-131.

[33] E. Tognoni, G. Cristoforetti, Signal and noise in laser induced breakdown spectroscopy: an introductory review, Opt. Laser Technol. 79 (2016) 164-172.

[34] J.H. Dillon, The modified Griess test: a chemically specific chromophoric test for nitrite compounds in gunshot residues, AFTE J. 22 (1990) 49-56.

[35] J.H. Dillon, The Sodium Rhodizonate test: a chemically specific chromophoric test for lead in gunshot residues, AFTE Journal. 22 (1990) 57-62.

[36] R. Nichols, Expectations regarding gunpowder depositions, AFTE Jl. 30 (1998) 94-101. 